\title{
Characterisation of the Antarctic stratospheric vortex mixing barrier
}

\author{
by
}

\section{Chris Cameron}

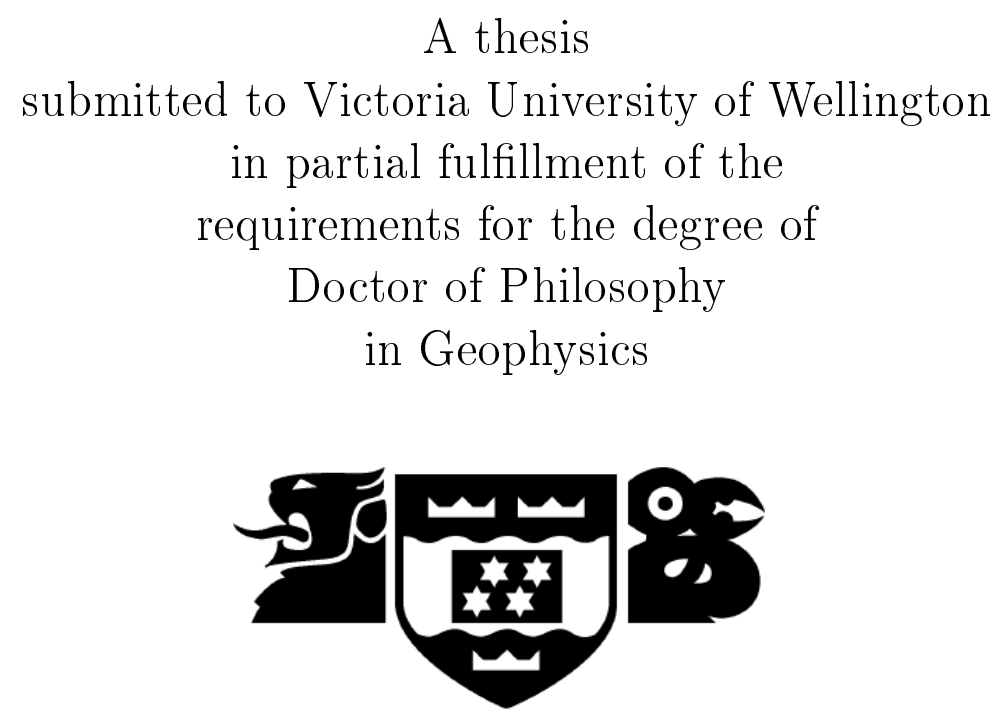

Victoria University of Wellington

2019 

Big whorls have little whorls which feed on their velocity and little whorls have lesser whorls

and so on to viscosity

Lewis Fry Richardson (1922) 


\begin{abstract}
The strongest stratospheric circulation in the Southern Hemisphere is the Antarctic Circumpolar Vortex (ACV) which forms each winter and spring as a zone of westerly winds surrounding Antarctica, presenting a barrier to transport of air masses between middle and high-latitudes. This barrier contributes to stratospheric temperatures above the polar region dropping sufficiently low in spring to allow for the processes leading to ozone destruction. Unfortunately the ACV is generally not well simulated in Global Climate Models (GCMs), and this presents a challenge for model accuracy and projections in the face of a changing climate and a recovering ozone hole.

In this research, an assessment is made of the performance of a range of mixing metrics in representing the ACV based on reanalyses, including: Effective Diffusivity, Contour Crossing, the Lagrangian function $M$, and Meridional Impermeability. It is shown that Meridional Impermeability - which provides a measure of the strength of the meridional mixing barrier as a function of potential vorticity (PV) gradient and wind-speed - acts as a useful proxy for more complex metrics. In addition, Meridional Impermeability displays a well-defined vortex profile across equivalent latitude, which is not seen to the same degree in the other metrics assessed.

Representation of the ACV is further compared between climate models and reanalyses based on Meridional Impermeability. It is shown that while climate models have improved in their representation of the vortex barrier over time, there are still significant discrepancies between models and reanalyses. One cause of these discrepancies may result from the use of prescribed ozone fields rather than interactive ozone chemistry. This is further examined by comparing Chemistry Climate Model (CCM) simulations using interactive ozone chemistry, with those using prescribed ozone at either 3-D (i.e., height, latitude and longitude) or 2-D (i.e., height, latitude) dimensionality.

Considerable improvement in the representation of the ACV can be achieved by shifting from 2-D to 3-D prescribed ozone fields, and interactive ozone chemistry further improves its representation. However, discrepancies in model representation of the ACV still remain. Previous researchers have also attributed discrepancies in model representation of the polar vortices to the model resolution, and the parameterization of gravity waves at the subgrid scale - these factors are considered to contribute to the discrepancies found in simulations undertaken here also.

The results of this research are expected to provide guidance to improve the representation of vortex processes in climate modelling.
\end{abstract}




\section{Contents}

List of Figures $\quad$ xi

List of Tables $\quad$ xiii

List of Acronyms $\quad$ Xv

Acknowledgements xvii

1 Introduction $\quad 1$

1.1 Overview ............................ 1

1.2 Thesis structure . . . . . . . . . . . . . 2

1.3 Research questions .................. 3

2 Background 5

2.1 Stratospheric circulation ............... 5

2.1.1 Transport, mixing and diffusion processes . . . . . . 6

2.1.2 Wave activity in the atmosphere . . . . . . . 8

2.1.3 Potential Vorticity . . . . . . . . . . . . 10

2.1.4 The Brewer-Dobson Circulation . . . . . . . . . . 12

2.1 .5 The $\mathrm{QBO} \ldots \ldots \ldots \ldots \ldots$

2.2 Southern Hemisphere circulation . . . . . . . . . . . 16

2.2.1 The Southern Annular Mode . . . . . . . . . . . 16

2.2.2 The Antarctic Circumpolar Vortex ......... . . . 18

2.2.3 The ACV as a transport barrier . . . . . . . . . . 19

2.2.4 ACV positive feedbacks . . . . . . . . . 21

2.2.5 Ozone in the stratosphere . . . . . . . . . . 22

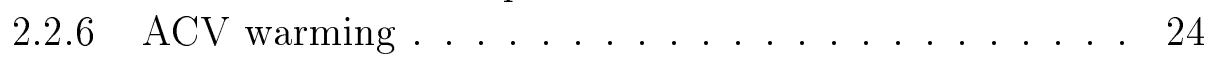

2.2.7 ACV asymmetry ....................... 26

2.3 Measurement of stratospheric state . . . . . . . . . . 28

2.4 Meteorological reanalyses . . . . . . . . . . . . . 29

2.4 .1 NCEP-CFSR . . . . . . . . . . 31

2.4 .2 ERA-Interim ....................... 31 
2.5 Global Climate Models . . . . . . . . . . . . . . . 31

2.5.1 Vertical interpolation . . . . . . . . . . 33

2.6 Mixing metrics . . . . . . . . . . . . . 34

3 Mixing metrics in the Stratosphere 35

3.1 Mixing metrics .................. 35

3.1 .1 Contour Crossing . . . . . . . . . . . . . 38

3.1 .2 Effective Diffusivity . . . . . . . . . . . 38

3.1.3 Meridional Impermeability . . . . . . . . . . 41

3.1 .4 Function $M \ldots \ldots \ldots . \ldots . \ldots 42$

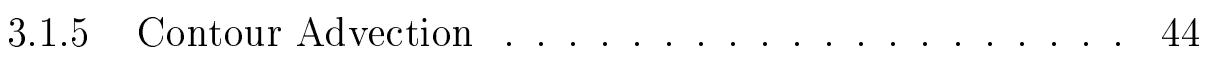

3.1.6 Lyapunov Exponents . . . . . . . . . . . . . . . . . 45

3.1.7 Lagrangian Coherent Structures . . . . . . . . . . . 46

3.2 Existing metric comparisons . . . . . . . . . . . . . 47

3.3 Analysis ........................ 48

3.3 .1 Trajectory model ...................... 51

3.4 Results . . . . . . . . . . . . . . 53

3.4.1 Meridional profiles . . . . . . . . . . 53

3.4.2 Metric correlations . . . . . . . . . . . 59

3.4.3 Other levels and reanalyses ................. 68

3.5 Discussion . . . . . . . . . . . . . . 69

3.6 Conclusions ......................... 72

4 The vortex barrier in the Unified Model 75

4.1 Methods .................... 76

4.1.1 The Unified Model . . . . . . . . . . . . 76

4.1.2 Model simulations . . . . . . . . . . . 78

4.2 Results . . . . . . . . . . . . . . . . . 79

4.2.1 Climatology . . . . . . . . . . . . 79

4.2.2 Monthly versus daily data ................ 83

4.2.3 Time series analysis . . . . . . . . . . . . . . 85

4.2.4 Vertical profiles .................... 90

4.3 Discussion and conclusions . . . . . . . . . . . 95

5 The vortex barrier in the SOCOL model 99

5.1 Ozone in climate models . . . . . . . . . . . . . . . 99

5.2 Methods . . . . . . . . . . . . . . . . 100

5.2 .1 The SOCOL model . . . . . . . . . . 100

5.2 .2 Model simulations . . . . . . . . . . 101

5.2 .3 Analysis .................... 102

5.3 Results . . . . . . . . . . . . . . . . . 102 
5.3 .1 Climatology . . . . . . . . . . . . . 102

5.3 .2 Monthly versus daily data . . . . . . . . . 105

5.3 .3 Time-series analysis . . . . . . . . . . . 105

5.3 .4 Vertical profiles ................ 109

5.3 .5 Temporal variation in $\kappa$ and ozone . . . . . . . . . 112

5.4 Discussion and conclusions . . . . . . . . . . . 117

6 Discussion $\quad 121$

$\begin{array}{llr}7 & \text { Conclusions } & 129\end{array}$

A Additional metric Figures 133

$\begin{array}{ll}\text { B Additional UM Figures } & 137\end{array}$

$\begin{array}{lr}\text { C Additional SOCOLv3 Figures } & 143\end{array}$

$\begin{array}{lr}\text { Bibliography } & 149\end{array}$ 


\section{List of Figures}

2.1 Isentropic surfaces $\ldots \ldots \ldots \ldots$

2.2 Potential Vorticity $(\mathrm{PV}) \ldots \ldots \ldots \ldots . \ldots . \ldots 11$

2.3 The Brewer-Dobson Circulation (BDC) . . . . . . . . 13

2.4 The Quasi-Biennial Oscillation (QBO) . . . . . . . . . 15

2.5 The Southern Annular Mode (SAM) . . . . . . . . . . 17

2.6 The Antarctic Circumpolar Vortex (ACV) . . . . . . . . . 20

2.7 Ozone concentrations 1980, 1999, $2014 \ldots \ldots \ldots$

2.8 Vortex asymmetry . . . . . . . . . . . . . . 27

2.9 Climate model evolution . . . . . . . . . . . . . 32

3.1 Meridional PV gradient profiles . . . . . . . . . . . 37

3.2 Loon balloon and RDF plot . . . . . . . . . . . . . 39

3.3 Bifurcated PV gradient (Conway et al., 2018) . . . . . . . . . 42

3.4 Function $M$ (Smith and McDonald, 2014) . . . . . . . . 43

3.5 LCS vortex representation (Haller, 2015) . . . . . . . . . . 46

3.6 Metric schematics . . . . . . . . . . . . . 49

3.7 Example of metric profiles by $\phi_{e} \ldots \ldots \ldots . . . . . . .52$

3.8 3-year metric means and standard deviations (NCEP-CFSR) . 54

3.9 Seasonal comparison of metrics (NCEP-CFSR) . . . . . . 56

3.10 Seasonal maximum metric values and their $\phi_{e}$ location . . . . 58

3.11 Metric correlations 1 (NCEP-CFSR) . . . . . . . . . 61

3.12 Metric correlations 2 (NCEP-CFSR) . . . . . . . 63

3.13 Metric correlations 3 (NCEP-CFSR) . . . . . . . . 65

3.14 Metric correlations 4 (NCEP-CFSR) . . . . . . . 67

4.1 UM vs reanalyses September climatology at $550 \mathrm{~K}$. . . . . 80

4.2 Daily and monthly $\kappa$ comparison ... . . . . . . . . 84

$4.3 \mathrm{UM}$ vs reanalyses September time series at $550 \mathrm{~K} \ldots . . .86$

$4.4 \mathrm{UM}$ vs reanalyses $\kappa$ pentads . . . . . . . . . . 88

4.5 UMETRAC $\kappa$ pentads (Struthers et al., 2009) . . . . . . 89

4.6 UM vs reanalyses climatological vertical profiles . . . . . . . 91

4.7 UM vs reanalyses PV gradient climatological cross-sections . . 92 
4.8 UM vs reanalyses $\kappa$ climatological cross-sections . . . . . . . 94

5.1 SOCOLv3 vs reanalyses Sept climatology at $550 \mathrm{~K}$. . . . . . . 103

5.2 Daily and monthly $\kappa$ comparison . . . . . . . . . 106

5.3 SOCOL vs Reanalyses time series at $550 \mathrm{~K} \ldots . . . . . .107$

5.4 SOCOLv3 vs NCEP-CFSR $\kappa$ pentads . . . . . . . . . 109

5.5 SOCOLv2 $\kappa$ pentads (Struthers et al., 2009) . . . . . . . 110

5.6 SOCOLv3 vs NCEP-CFSR $\kappa$ climatological cross-sections . . . 111

5.7 Full $\kappa$ and ozone time-series . . . . . . . . . . . . . 113

5.8 Regression relationship in $\kappa^{\prime}$ and OMD' . . . . . . . . . 116

5.9 Ozone anomaly time-series . . . . . . . . . . . 117

A.1 Seasonal comparison of metrics at $550 \mathrm{~K}$ (ERA-Interim) . . . . 134

A.2 Seasonal comparison of metrics at $850 \mathrm{~K}$ (ERA-Interim) . . . 135

A.3 Seasonal comparison of metrics at $850 \mathrm{~K}$ (NCEP-CFSR) . . 136

B.1 UM vs reanalyses July climatology at $550 \mathrm{~K}$. . . . . . . . . 138

B.2 UM vs reanalyses August climatology at $550 \mathrm{~K}$. . . . . . . 139

B.3 UM vs reanalyses July climatology at $850 \mathrm{~K}$. . . . . . . . 140

B.4 UMvs reanalyses August climatology at $850 \mathrm{~K}$. . . . . . . . 141

B.5 UM vs reanalyses Sept climatology at $850 \mathrm{~K} \ldots . . . . . .142$

C.1 UM vs reanalyses July climatology at $550 \mathrm{~K}$. . . . . . . . 144

C.2 UM vs reanalyses Aug climatology at $550 \mathrm{~K} \ldots . . . . . .145$

C.3 UM vs reanalyses July climatology at $850 \mathrm{~K} \ldots . . . . . .146$

C.4 UM vs reanalyses Aug climatology at $850 \mathrm{~K} \ldots . . . . . .147$

C.5 UM vs reanalyses Sept climatology at $850 \mathrm{~K} \ldots . . . . .148$ 


\section{List of Tables}

2.1 U.S. Standard Atmosphere . . . . . . . . . . . . 7

2.2 Examples of modern reanalyses . . . . . . . . . 30

3.1 Mixing diagnostics investigated . . . . . . . . . . . 50

3.2 Spearman correlation in mixing diagnostics . . . . . . . 66

3.3 Seasonal patterns in mixing diagnostics . . . . . . . . . . 69

3.4 Strengths and weaknesses in mixing diagnostics . . . . . . 71

4.1 UM configurations assessed . . . . . . . . . 76

4.2 UM climatological metric results . . . . . . . . . . . . 81

5.1 Curve metrics (SOCOLv3) . . . . . . . . . . . 104 


\section{List of Acronyms}

\begin{tabular}{|l|l|} 
ACV & Antarctic Circumpolar Vortex \\
BDGCM & Atmosphere Ocean General Circulation Model \\
CCM & Brewer-Dobson Circulation \\
CCMI & Chemistry-Climate Model \\
CFSR & Chemistry-Climate Model Initiative \\
ECMWF & Elimate Forecast System Reanalysis \\
ED & Effective Diffusivity \\
EL & Equivalent Length \\
ERA-Interim & European Reanalysis-Interim \\
ESM & Earth System Model \\
ETH & Swiss Federal Institute of Technology \\
FSLE & Finite Size Lyapunov Exponents \\
FTLE & Finite Time Lyapunov Exponents \\
FWHM & Full Width at Half Maximum \\
GA & Global Atmosphere \\
GCM & Global Climate Model \\
GHG & Greenhouse Gas \\
GWD & Gravity Wave Drag \\
HadGEM & Hadley Centre Global Environmental Model \\
IPCC & Intergovernmental Panel on Climate Change \\
LCS & Lagrangian Coherent Structure \\
LIDAR & Light Detecting and Ranging \\
Loon & Google[x] Project Loon \\
MA-ECHAM & Middle-Atmosphere European Centre/Hamburg Climate Model \\
MERRA & Modern Era Retrospective-analysis for Research and Applications \\
NASA & National Aeronautics and Space Administration \\
NCAR & National Center for Atmospheric Research \\
NCEP & National Centers for Environmental Prediction \\
NH & Northern Hemisphere \\
NIWA & National Institute of Water and Atmospheric Research \\
&
\end{tabular}




\begin{tabular}{l|l} 
NOAA & National Oceanic and Atmospheric Administration \\
NOGWD & Non-Orographic Gravity Wave Drag \\
NWP & Numerical Weather Prediction \\
NZESM & New Zealand Earth System Model \\
OGWD & Orographic Gravity Wave Drag \\
PSC & Polar Stratospheric Cloud \\
PV & Potential Vorticity \\
PVU & Potential Vorticity Units \\
QBO & Quasi-Biennial Oscillation \\
RCM & Regional Climate Model \\
RCP & Representative Concentration Pathway \\
RDF & Reverse Domain Filling \\
SAM & Southern Annular Mode \\
SH & Southern Hemisphere \\
SOCOL & Solar Climate Ozone Links \\
SPARC & Stratospheric Processes and their Role in Climate \\
SST & Sea Surface Temperature \\
SSW & Sudden Stratospheric Warming \\
UCAR & University Corporation for Atmospheric Research \\
UKCA & UK Chemistry and Aerosols model \\
UM & Unified Model \\
UMETRAC & Unified Model with Eulerian Transport and Chemistry \\
UV & Ultraviolet \\
WMO & World Meteorological Organisation
\end{tabular}




\section{Acknowledgements}

I would like to heartily acknowledge the support of my supervisors, Greg Bodeker and James Renwick. Greg was consistently able to explain complex concepts in a way that was both entertaining and effective. James was always on hand when needed, and would frequently offer to shout a drink and have a chat - needless to say that such kind offers were generally taken up and very much appreciated.

Thanks to my co-authors in papers submitted during the course of my research. These of course include my supervisors, but also Jono Conway, Stephen Stuart, Laura Revell and Andrea Stenke. The access to the highperformance computing facilities at ETH, Zurich (via Laura and Andrea), and the NIWA super-computing facility in Wellington (via Stephen) were invaluable. Thanks also to colleagues at Bodeker Scientific who offered various sage words of advice or invaluable support along the way.

Funding was provided by the Marsden Fund (contract BDS1401) of the Royal Society of New Zealand. In addition, I was supported by Victoria University of Wellington with a 'Thesis completion grant', to assist in expenses during the final months of this research.

Finally, thank-you to my wife Carolyn and our children - Maz, Fern and Arlo - who were supportive and encouraging, if somewhat mystified by what this was all about. 


\section{Chapter 1}

\section{Introduction}

\subsection{Overview}

The stratosphere plays a key role in Earth's climate system, including through its influence on the transport of particulates and trace gases from the tropics to the polar regions, winter-time isolation of air above the pole, ozone creation and destruction, and connections between stratospheric dynamics and weather in the troposphere.

A large body of evidence has accumulated that highlights the importance of stratospheric change in driving changes in climate at the Earth's surface (e.g., Solomon et al. 1985). This is particularly important for middle and high latitudes in the Southern Hemisphere (SH), where stratospheric ozone depletion has driven changes in surface climate. The effects of changing dynamics in the stratosphere will continue to be of interest in the future as the ozone layer over Antarctica recovers from the effects of ozone depleting substances (ODSs) as their concentrations decline, and as $\mathrm{CO}_{2}$ and other greenhouse gases (GHGs) continue to accumulate in the atmosphere (Butchart et al., 2000).

While ongoing improvement has been seen in Global Climate Model (GCM) development, there are shortcomings in the ability of these models to accurately simulate processes in the stratosphere, particularly in the SH polar region where measurements are scarce (e.g., Jones et al. 2016).

The Antarctic Circumpolar Vortex (ACV) forms in the SH stratosphere each winter, and acts as a barrier to meridional transport. Imprecise representation of processes that determine the strength and structure of the ACV lead to its incorrect simulation in GCMs, including Chemistry Climate Models (CCMs) (e.g., Hardiman et al. 2017). It is important that climate models simulate this barrier well as it determines spatial gradients in radiatively active gases, such as ozone, which then determine the spatial morphology of 
the radiative forcing field.

To address such deficiencies, our understanding of large-scale transport processes in the stratosphere needs to be advanced (e.g., Karpechko et al. 2013). In addition, gaps remain in our understanding of the dynamical processes controlling the impermeability of the ACV (SPARC, 2010).

The current research aims to address some of these shortcomings and, in particular, investigate aspects of the dynamics of the ACV mixing barrier, including its impermeability. First, a range of diagnostic metrics for defining the mixing barrier are assessed, with the aim of determining their effectiveness at describing the vortex meridional transport barrier, and establishing if a single metric may be preferred over others. Then, a more in-depth assessment is undertaken to determine how well the ACV is represented in climate models.

\subsection{Thesis structure}

The overview provided above has established the motivation for the research undertaken here. The remainder of this chapter describes the structure of the thesis, and outlines the fundamental research questions.

Chapter 2 presents an introduction to the global stratosphere and relevant processes within it, outlining the current state of knowledge and indicating relevant research needs. The chapter commences with an overview of Earth's atmospheric circulation, with an emphasis on stratospheric transport and mixing processes, before turning attention to the $\mathrm{SH}$ stratosphere, the causes and characteristics of the ACV, and its relationship to the ozone hole and increasing concentrations of GHGs. Finally, approaches to measurements, modelling and reanalyses are discussed.

Each of the chapters that then follow commence with a brief overview before describing the methods, results, implications and relevance of the research relating to that chapter topic.

Chapter 3 examines a range of mixing metrics in the stratosphere and compares their performance and utility in defining mixing in the $\mathrm{SH}$ polar region, including how well they represent the ACV mixing barrier. Selected metrics are examined in-depth, including Meridional Impermeability, Effective Diffusivity, Function $M$, and Contour Crossing.

Chapter 4 provides an assessment of how well a GCM performs in its representation of the vortex mixing barrier, compared against reanalyses, with the aim of determining if and where improvements may be required in climate models. The simulation of ACV impermeability in this chapter is based on the Meridional Impermeability metric. Two configurations of the 
UK Met Office Unified Model (UM) are used, each representing different generations of UM advancement.

Chapter 5 examines the vortex mixing barrier as simulated by a CCM, specifically investigating the difference between model simulations using interactive ozone chemistry, and those where ozone is prescribed in either 2-D or 3-D form. Previous work has identified that the way in which ozone is prescribed in climate models can have a considerable influence on resultant dynamical fields, and thereby on the strength and form of the ACV in the stratosphere. Meridional Impermeability is again used as the key metric for which representation of the ACV is assessed.

Chapter 6 provides a discussion that aims to synthesise the content of preceding chapters, locate the relevance of this research in the context of previous work, and provide direction for future research.

Finally, Chapter 7 presents the conclusions, summarising the work undertaken across the entire thesis.

\subsection{Research questions}

The brief overview above establishes the motivation for the research undertaken in this thesis. While it was anticipated that data that were made available from Loon balloon trajectories (see section 2.3 on stratospheric measurement) could be used to investigate the impermeability of the ACV, those data were found to be ill-suited for that purpose. The work of this thesis instead became focused on large-scale transport in the stratosphere and model representation of the ACV transport barrier. To provide direction for the subsequent chapters, the following research questions were developed:

1. What is the ACV, and what role does it play in the stratosphere?

2. What are the processes that determine the dynamical attributes of the $\mathrm{ACV} ?$

3. What processes relevant to the dynamical attributes of the ACV may be missing or ill-defined in GCMs? 


\section{A note on papers prepared for publication}

Chris Cameron was the lead author for three manuscripts which provide the basis for individual chapters in this thesis:

\section{Chapter 3}

Cameron, C., Conway, J.P., Bodeker, G.E., Renwick, J. An assessment of stratospheric mixing diagnostics in the Antarctic circumpolar vortex region, Atmospheric Chemistry and Physics. Paper in draft form, not submitted.

\section{Chapter 4}

Cameron, C., Bodeker, G., Conway, J., Stuart, S. and Renwick, J. (2019) Simulating the Antarctic stratospheric vortex transport barrier: comparing the Unified Model to reanalysis., Climate Dynamics. 10.1007/s00382-0184593-5.

\section{Chapter 5}

Cameron, C., Bodeker, G.E., Conway, J.P., Revell, L.E., Andrea, S., and Renwick, J. (rev.) Comparing the effects of the dimensionality of prescribed and interactive ozone on the fidelity of the Antarctic stratospheric vortex in a global climate model, Geophysical Research Letters. Under review. 


\section{Chapter 2}

\section{Background}

This chapter provides an overview of atmospheric processes and transport in the stratosphere, including review of existing literature and a description of the renalyses and climate models used in later chapters.

\subsection{Stratospheric circulation}

Located directly above the troposphere and tropopause (Figure 2.1), the stratosphere has an average depth of $\sim 38 \mathrm{~km}$ compared with an average depth of $\sim 12 \mathrm{~km}$ for the troposphere (e.g, Andrews et al. 1987). The total mass of the troposphere is much higher than that of the stratosphere, and the troposphere contains the vast majority of the water vapour in the atmosphere (Holton et al. 1995; Kidston et al. 2015).

The troposphere is shallower at the poles and deeper near the equator (Figure 2.1) due to a warmer and less dense air column in the tropics, while colder, denser air pervades in the polar regions (Holton et al., 1995). However, the middle and upper stratosphere are of relatively constant depth across all latitudes with low density and high static stability which reduces vertical mixing. The lower-most stratosphere extends between the poles and the tropics, but does not cross the equator, and contains potential temperature surfaces (see section 2.1.1 below) which cross the tropopause. Above the stratosphere the variation of atmospheric height with latitude returns where, for example, the mesopause is higher in the tropics but lower in the polar region of the summer hemisphere due to adiabatic processes in the mesosphere (Xu et al., 2007).

Approximate associations of altitude $(\mathrm{km})$, pressure $(\mathrm{hPa})$, temperature $(\mathrm{K})$ and potential temperature $(\mathrm{K})$ are shown for the US Standard Atmosphere in Table 2.1 . 


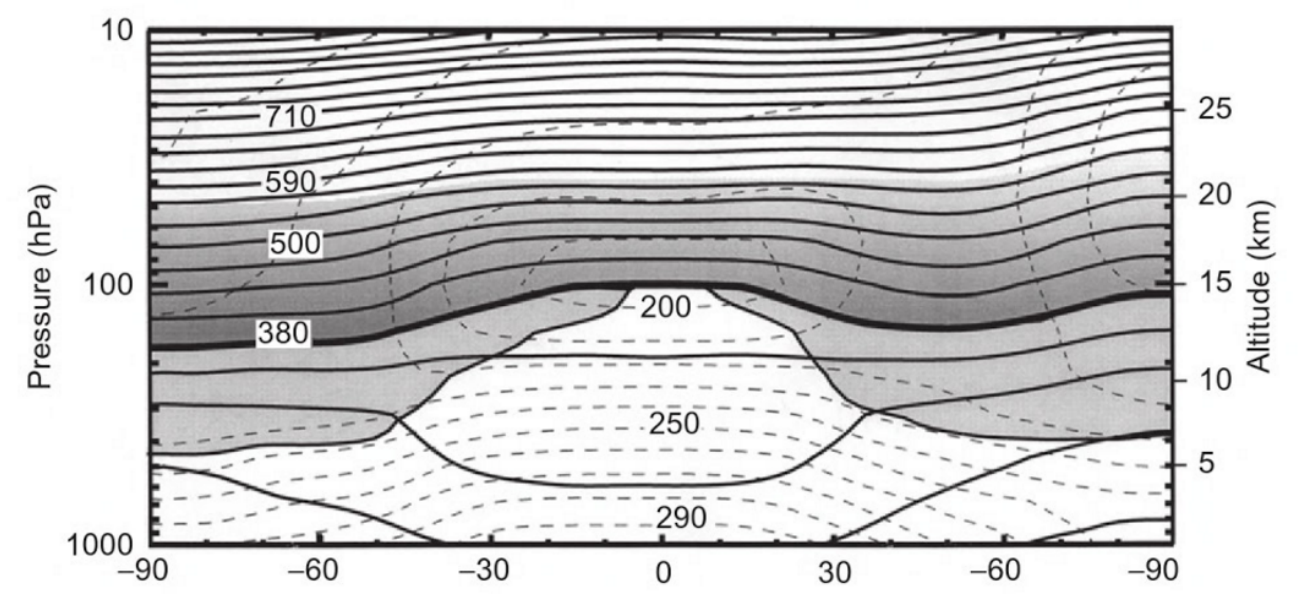

Figure 2.1: January 1993 mean potential temperature $(\theta)$ surfaces (solid contours at $30 \mathrm{~K}$ intervals) and temperature (dashed contours at $10 \mathrm{~K}$ intervals) with latitude and altitude. The heavy line is the $380 \mathrm{~K} \theta$ surface and the light shading indicates the lower-most stratosphere, where $\theta$ surfaces cross the tropopause. Source: Figure 12.17 in Holton (2004).

\subsubsection{Transport, mixing and diffusion processes}

Transport, diffusion and mixing processes in the atmosphere occur at a range of spatial scales, caused by stirring and wave action driven by the thermodynamics and rotation of the Earth (Holton, 2004). Atmospheric transport can be defined as the advection of an air parcel by the ambient wind-field, while diffusion is the dispersal of a substance (or an air parcel) from an area of high to low concentration at a molecular level, ultimately resulting in irreversible transport (Holton, 2004). The rate of molecular diffusion in the atmosphere (i.e., the change in concentration per unit of air over a selected time interval) is a function of the temperature of an air parcel and increases with altitude, varying inversely with pressure.

Stirring can lead to fine filaments of air being extruded from the flow. This filamentation process may result in air parcels from entirely different source areas with very different initial properties becoming located in close proximity. The differing air parcel properties may include their Potential Vorticity (PV - see section 2.1.3 below) or their concentrations of trace gases (Polvani and Plumb 1992; Plumb 2007). Exponential growth in filamentation or material stretching can rapidly mix air parcels (Chen, 1994) which are ultimately diffused at small scales.

Diabatic processes in the atmosphere involve a transfer of energy between an air parcel and its surroundings through heating or cooling (Holton, 2004). 


\begin{tabular}{|r|r|r|r|}
\hline $\begin{array}{r}\text { Height } \\
(\mathrm{km})\end{array}$ & $\begin{array}{r}\text { Pressure } \\
(\mathrm{hPa})\end{array}$ & $\begin{array}{r}\text { Temperature } \\
(\mathrm{K})\end{array}$ & $\begin{array}{r}\text { Potential } \\
\text { temperature }(\mathrm{K})\end{array}$ \\
\hline 0 & 1013 & 288.1 & \\
1 & 899 & 281.7 & 275 \\
10 & 265 & 223.3 & 400 \\
15 & 121 & 216.6 & 550 \\
20 & 55 & 216.6 & 650 \\
25 & 25 & 221.7 & 800 \\
30 & 12 & 226.7 & 1300 \\
40 & 3 & 251.1 & 2100 \\
50 & 0.8 & 270.7 & 5800 \\
70 & 0.1 & 217.5 & \\
\hline
\end{tabular}

Table 2.1: Selected values of pressure and temperature with height from the 1976 U.S. Standard Atmosphere (NASA, 1976), together with potential temperature estimated following Knox (1998) using $\theta=z \times 27.75$ from $10 \mathrm{~km}$ to $30 \mathrm{~km}$ and then $\theta=555 e^{0.05(z-23)}$ above $30 \mathrm{~km}$, with values rounded to the nearest $50 \mathrm{~K}$.

Examples include radiative heating or cooling, heating which results from molecular viscosity or chemical processes, and heat fluxes from the surface of the Earth (Shepherd, 2003). Adiabatic processes occur where the air parcel does not undergo transfer of energy with its surroundings and the potential temperature of the air parcel is conserved (Shepherd, 2003).

An isentropic surface is characterised by having constant potential temperature, or constant entropy - where potential temperature is defined as the temperature that an air parcel would have if it were adiabatically brought to a near-surface (generally $1000 \mathrm{hPa}$ is used) reference point (Holton, 2004). Transport in the atmosphere can be categorised as either isentropic - movement adiabatically along isentropic surfaces, or anisentropic - diabatic movement across isentropic surfaces (Haynes and Shuckburgh, 2000). In the stratosphere, and for atmospheric transport over a few days, mixing can be assumed to occur adiabatically along isentropic surfaces, with diabatic changes neglected (Holton, 2004). While diabatic processes typically occur over periods of several weeks in the lower stratosphere, isentropic transport typically occurs over periods of 5-10 days (Holton et al., 1995). Isentropic mixing is a key component of global mass transport in the stratosphere and has a large effect on the distribution of chemical species.

The assumption of adiabatic transport does not hold true at isentropic levels in the troposphere, which tend to intersect with the Earth's surface 
(Figure 2.1), making it difficult to calculate transport and diffusion rates (Chen, 1994). In addition, in the mid-latitudes of the lowermost stratosphere, potential temperature surfaces transect the tropopause where diabatic transport occurs (see Figure 2.1). For the middle-stratosphere and above the isentropes become quasi-horizontal, which allows potential temperature to become an important vertical co-ordinate for stratospheric applications (Holton, 2004).

Although air in the stratosphere is of much lower density than air in the troposphere, it can have a strong influence on weather near the Earth's surface via so-called 'stratosphere-troposphere coupling' (e.g., Holton et al. 1995; Baldwin and Dunkerton 1999; Thompson and Solomon 2002; Kidston et al. 2015). Gerber (2012) notes that this coupling occurs across a range of spatial and temporal scales. Dynamic coupling occurs between the circumpolar jet in the winter stratosphere and atmospheric transport in the troposphere with stronger wind in the stratospheric jet resulting in a poleward shift in the tropospheric jet stream (Kidston et al., 2015). It is also now understood that the stratosphere plays an important role in transmitting the ENSO signal to mid-latitudes (Bell et al., 2009), and in determining the tropospheric response to stratospheric volcanic aerosols (Gerber, 2012). However, the detailed mechanisms connecting the dynamics of the stratosphere and the troposphere are not fully understood (Kidston et al., 2015).

Stratosphere-troposphere coupling also occurs in spring and summer in the SH when weaker westerly winds prevail and planetary waves can propagate more easily into the stratosphere (Kidston et al., 2015). Because stratosphere-troposphere coupling is important for simulating the climate of SH mid-latitudes, it is particularly important that coupling processes are simulated with high fidelity in GCMs. In recognition of this need, models are increasingly raising their vertical extent, assimilating data higher into the stratosphere, adding additional layers and including additional stratospheric processes (Gerber, 2012).

\subsubsection{Wave activity in the atmosphere}

Planetary waves (also known as Rossby waves) are large-scale waves with wavelengths similar to the Earth's circumference, and play a critical role in coupling the lower and upper atmosphere (Andrews et al., 1987). These waves are generated in the troposphere by thermal and orographic forcing, and primarily driven by planetary rotation and the Coriolis force (i.e., planetary vorticity) (Andrews et al., 1987).

As they propagate upward, the largest-scale planetary waves grow in amplitude and are refracted equatorward. At high amplitudes these waves 
weaken the mean flow and may then break equatorward of the circumpolar vortex (see section 2.2.2 below for further details on the vortex) in the winter hemisphere's stratospheric mid-latitude 'surf zone' - a large scale region of planetary wave-breaking (McIntyre and Palmer, 1984).

When planetary wave-breaking occurs at the equatorward edge of the vortex, filaments of air are eroded from the vortex and mixed with mid-latitude air (Juckes and McIntyre, 1987). Planetary wave breaking can thereby lead to irreversible mixing, erosion of the vortex edge (McIntyre and Palmer, 1984), and deceleration of the stratospheric jet, sometimes to the extent that winds reverse to become easterly (Kidston et al., 2015). While planetary waves play a key role in modulating the polar vortices, their wave-breaking action is also the primary driver of the Brewer-Dobson circulation (BDC) (e.g., Haynes et al. 1991; Holton et al. 1995; Thompson and Solomon 2002) - see section 2.1.4 below.

Planetary waves in the SH stratosphere are largest in amplitude in late autumn and early spring and at a minimum in summer, while mid-winter also sees low wave activity due to a maximum in zonal wind (Plumb, 2010). The springtime maximum in wave activity is typically larger than that in autumn, and corresponds to the peak in wave-breaking as the vortex dissipates at the end of the season as the equator to pole temperature gradient collapses (McIntyre and Palmer, 1983). Stronger planetary wave activity implies a faster meridional circulation, with a weaker vortex and more rapid horizontal mixing (Shepherd, 2003).

Gravity waves (GW) are generated by vertical uplift, where gravity is the restoring force, and act over a smaller scale than planetary waves (Alexander, 2010). Gravity waves may be orographic (OGWs) - such as those generated from uplift occurring over mountain ranges, or non-orographic (NOGWs) - such as those generated by strong convection (Alexander, 2010). While Plumb (2010) notes that planetary waves control most aspects of the circulation in the extratropical stratosphere, GWs also play an important role, particularly in the summer hemisphere, where they can account for much of the wave-driving in the stratosphere (Alexander, 2010). Dunkerton (1997) found that GWs contribute to the oscillation of the QBO (see section 2.1.5 below). Hendricks et al. (2014) find that anomalous GW amplitudes in the Atlantic and Indian Ocean sectors of the Southern Ocean are likely to be caused by NOGWs associated with winter storm tracks, the mid-latitude winter jet, frontogenesis, and convection.

Wave drag is the transfer of angular momentum induced by wave action, and leads to changes in dynamics that directly influence the transport of GHGs and ODSs in the atmosphere (Podglajen et al., 2016). Small scale 
GWs are not resolved in climate models (i.e., they occur at a finer scale than the grid-size of the model), so they are instead parameterized (Alexander, 2010). McLandress et al. (2012) find that insufficient OGW drag in climate models is most likely to be the cause of temperature and wind biases.

Increases in GHG forcing is expected to result in changes in the location, timing and influence of GWs, as differing rates of change in warming are expected in tropical regions and polar regions (Santer et al., 2005). For this reason, a current focus of climate modellers is to improve the parameterization of gravity waves - particularly NOGWs, given that these are non-stationary (Alexander, 2010).

\subsubsection{Potential Vorticity}

The utility of potential vorticity (PV) as a diagnostic of stratospheric dynamics has been demonstrated by many researchers (e.g., McIntyre and Palmer 1984; Hoskins et al. 1985). In particular, PV gradients provide the restoring mechanism for Planetary waves, meaning that the PV distribution is a key factor in understanding the dynamics of the atmosphere (Hoskins et al., 1985).

PV can be derived from momentum and thermodynamic equations, where PV is an analogue of angular momentum in the stratosphere, and is a quasiconserved property of an air parcel for inviscid and adiabatic flow (Haynes and McIntyre 1987; Holton 2004). The conservation of PV allows it to be used to track atmospheric transport and the evolution of the vortex (Waugh and Polvani, 2010).

$$
P V \equiv-g\left(\zeta_{\theta}+f\right)\left(\frac{\partial \theta}{\partial p}\right)
$$

Where $g$ is acceleration due to gravity, $\zeta_{\theta}$ is relative isentropic vorticity, $f$ is the Coriolis parameter (the spin imparted by the Earth's rotation, which varies as a function of latitude - otherwise planetary vorticity), $\theta$ is the potential temperature and $p$ is the pressure. Thus PV can be examined as the product of two main elements:

1. Dynamical element: Absolute isentropic vorticity $\left(\zeta_{\theta}+f\right)$. Relative vorticity $\left(\zeta_{\theta}\right)$ determines the spin imparted to an air parcel by curved flow:

$$
\zeta_{\theta}=\nabla \times \vec{v}
$$

Where $\vec{v}$ is the total horizontal wind vector. Absolute vorticity is therefore a function of the total horizontal wind-velocity gradient (Hoskins 

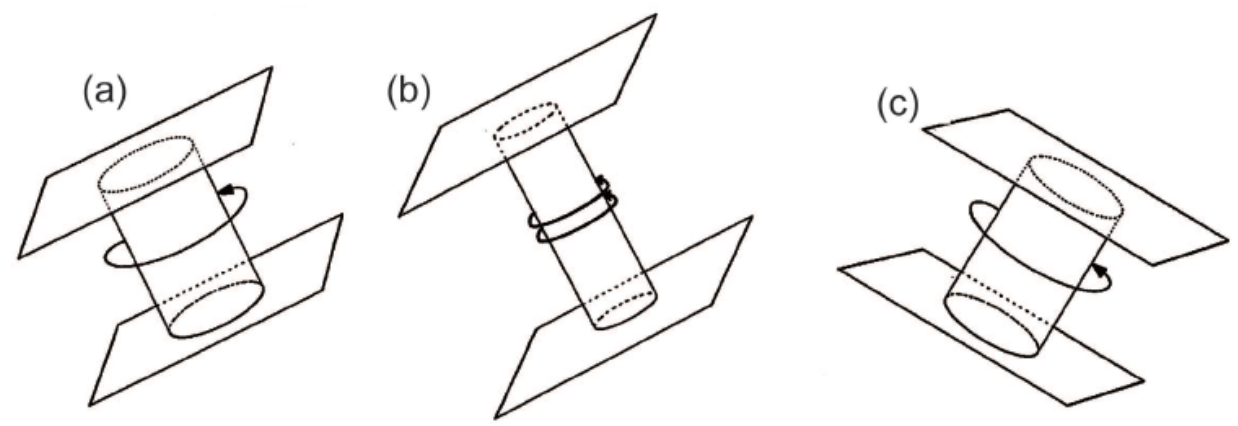

Figure 2.2: Schematic of (a) a rotating cylinder representing a column of air between two isentropic surfaces, (b) stretching and isentropic surface separation causing accelerated rotation, and (c) isentropic surface tilting. PV (shown by the arrows) is conserved in each case. Source: Hoskins (2015).

et al., 1985).

2. Thermodynamic element: Static stability $(-g \cdot \partial \theta / \partial p)$. This component describes the potential temperature lapse rate related to the spacing (or depth) between isentropic surfaces (i.e., the gradient of potential temperature with pressure).

Detailed discussion of the attributes of PV is provided by Haynes and McIntyre (1987), and more recently by Hoskins (2015). An important attribute of potential vorticity is its invertibility (Hoskins, 2015) - that is, if the spatial distribution of PV and boundary conditions are known then the complete flow structure can be deduced. Creation or destruction of PV occurs when diabatic processes drive changes in the energy content of the air parcel, e.g., through friction, uptake/release of latent heat, or absorption/emission of radiation (Haynes and McIntyre, 1987).

In the stratosphere it is found that PV is conserved despite changes in the depth or tilt of the bounding isentropic surfaces, as shown in Figure 2.2. In the case of stretching or separation of the bounding isentropic surfaces (Figure 2.2b) the rate of spin increases as the radius of the indicative air parcel decreases (Hoskins et al., 1985). In the case of tilting of the isentropic surfaces, the rate of spin remains the same, and the plane on which the spin of PV occurs remains normal to the isentropic surfaces. PV is usually expressed in PV units (PVU), where $1 \mathrm{PVU}=10^{-6} \mathrm{~K} \cdot \mathrm{m}^{2} \cdot \mathrm{kg}^{-1} \cdot \mathrm{s}^{-1}$. By convention $\mathrm{PV}$ is negative in the $\mathrm{SH}$.

The equivalent latitude $\left(\phi_{e}\right)$ associated with a PV value, first described by McIntyre and Palmer (1984) and developed further by Butchart and Rems- 
berg (1986) and Nash et al. (1996), is a Lagrangian coordinate that represents the true latitude that would enclose the same area as a selected PV isoline (Añel et al., 2013). Equivalent latitude allows for analysis of spatial changes in the PV distribution, and can be considered as indicating lines of material transport (Butchart and Remsberg, 1986). Equivalent latitude can also be used as a meridional co-ordinate to define the polar vortex (see section 2.2.2 below) based on a selected PV bounding value.

The calculation of $\phi_{e}$ has been found to become less valid at higher isentropic levels (Allen and Nakamura, 2003) as the quality of winds in models and reanalysis tends to degrade with altitude (Manney et al., 1996). Harvey et al. (2009) showed, using output from an assimilating model, that anomalously low PV values can be located inside the vortex annulus at levels down to $40 \mathrm{~km}$ (or an approximate isentropic level of $1300 \mathrm{~K}$ ) mostly due to static stability anomalies generated by temperature fluctuations.

Mapping PV to $\phi_{e}$ can also be problematic on the quasi-horizontal plane (i.e., isentropically), where areas of displaced high (absolute) PV lead to locations in the circumpolar wind-speed jet being erroneously mapped to lower equivalent latitudes, and vice-versa for areas of displaced low PV (Allen and Nakamura, 2003).

\subsubsection{The Brewer-Dobson Circulation}

The stratosphere plays a key role in the transport and dispersal of longlived trace gases in the atmosphere (Labitzke and Van Loon, 1999). The mass circulation of tropospheric air through the stratosphere is known as the Brewer-Dobson circulation (BDC) - or synonymously the diabatic circulation or the meridional overturning circulation (Cohen et al. 2014; Linz et al. 2017).

The BDC describes air rising above the tropopause at tropical latitudes (Figure 2.3) into the stratosphere before moving poleward and descending at middle and high latitudes (Butchart, 2014). The overturning time taken for this circulation is in the order of five years (Shepherd, 2003). Four key regions of air mass transport occur within the BDC (Haynes and Shuckburgh, 2000), and are apparent in Figure 2.3:

- The polar vortex $\left(\sim 60^{\circ}\right.$ to $90^{\circ}$ in the winter hemisphere)

- The surf-zone $\left(\sim 20^{\circ}\right.$ to $60^{\circ}$ in the winter hemisphere)

- The tropical uplift zone (crossing the equator at $\sim \pm 20^{\circ}$ )

- The summer extra-tropics $\left(\sim 20^{\circ}\right.$ to $90^{\circ}$ in the summer hemisphere)

Differing transport patterns are thus apparent in the winter and summer hemispheres, with increased wave-breaking and formation of the polar vortices (see section 2.2.2 below) in the winter hemisphere, and weak easterlies 


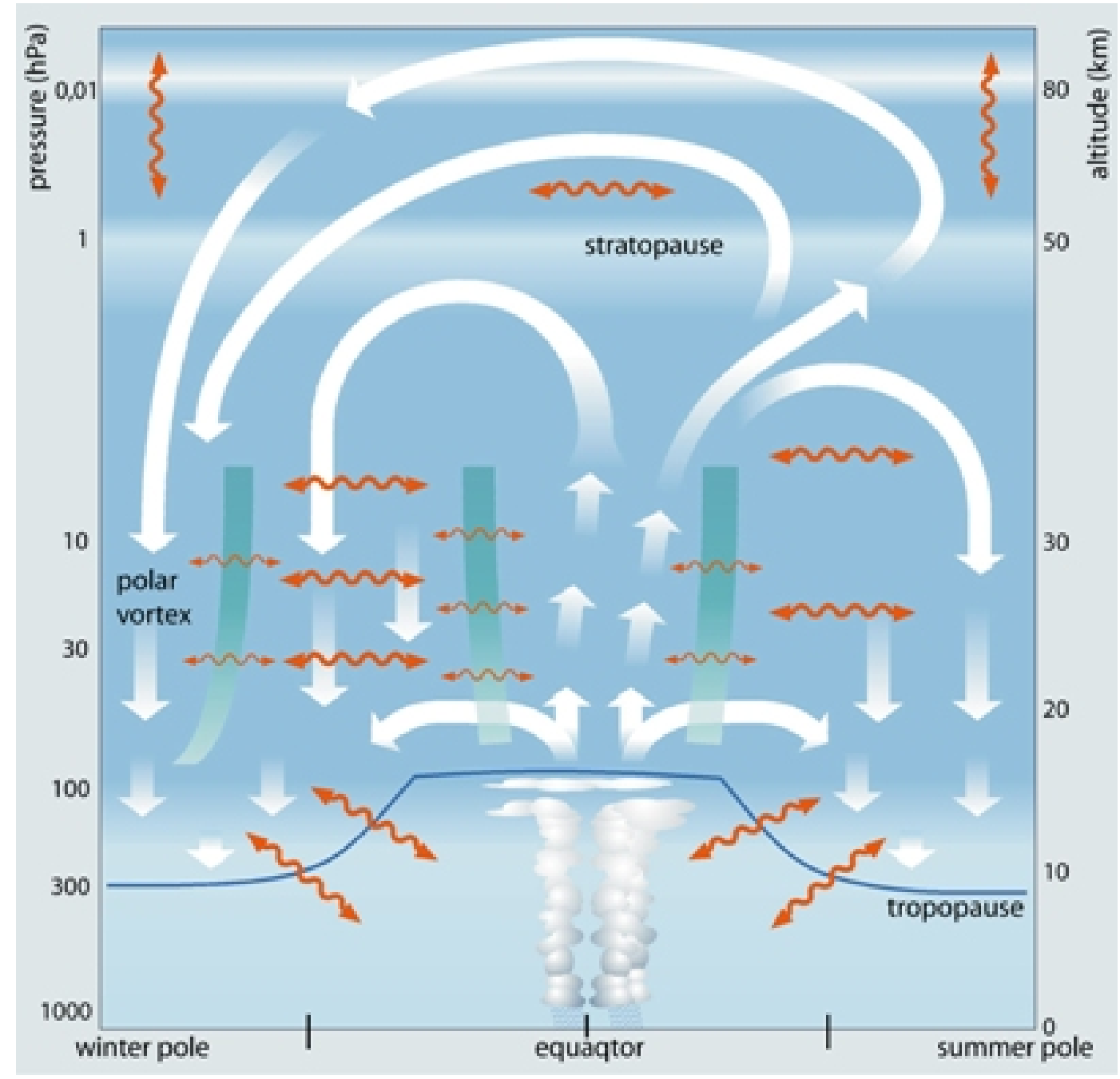

Figure 2.3: The Brewer-Dobson Circulation, showing tropospheric air masses entering the stratosphere via the tropical tropopause, rising through the stratosphere and moving poleward, before descending in extra-tropical and polar regions (white arrows). The blue near-vertical segments separate the key regions in the stratosphere, and from left to right these are: the winter polar vortex, the surf-zone, the tropical uplift zone, and the summer extra-tropics. Eddy transport from isentropic mixing is shown by the orange arrows. Source: Goethe University (goethe-universityfrankfurt.de/69128060/Atmospheric-Transport). 
in the summer hemisphere (Waugh and Polvani, 2010). The BDC is driven by the so-called 'downward control' process (Haynes et al., 1991), whereby planetary wave-breaking in the stratosphere decelerates the zonal wind and enhances poleward transport (Butchart, 2014).

While some researchers have found that the BDC has strengthened since the late 1970s, consistent with ozone loss (Thompson and Solomon, 2009), the evidence for this is sparse (Butchart, 2014). In model projections, simulations indicate that the BDC will accelerate by $\sim 2-3 \%$ per decade (depending on the GHG scenario used) over the course of the $21^{\text {st }}$ century due to climate change. This is expected to result in increased transport of ozone to the mid-latitudes, quicker removal of ODSs and changes in rates of stratospheretroposphere exchange (Butchart, 2014).

\subsubsection{The QBO}

The quasi-biennial oscillation (QBO) in the tropics plays an important role in the exchange of energy and trace gases between the upper and lower stratosphere (Baldwin et al., 2001). It is considered to be the most striking example of wave-driven, low-frequency variability in the stratosphere (Thompson and Solomon, 2002), and is driven by a range of wave types, including equatorial Kelvin waves, Rossby-gravity waves and gravity waves (Dunkerton, 1997). The QBO manifests as a roughly biennial oscillation (with an average cycle of approximately 28 months) of equatorial zonal winds between westerlies and easterlies, which propagate downward in the stratosphere (Baldwin et al., 2001).

Though the QBO is located in the tropics, it effects the entire stratospheric global circulation by modulating upward propagating extratropical waves (Holton and Tan, 1980). In its easterly phase, the QBO weakens the polar vortex by diffracting planetary waves poleward (Baldwin et al. 2001; Thompson and Solomon 2002), and vice-versa. The phase of the QBO determines the location of the critical region (or zero wind line), which in turn controls the location of the surf zone in the stratosphere (Holton and Tan, 1980).

An unexpected anomaly in the downward propagation of the westerly winds of the QBO was observed for the first time in 2016 (Figure 2.4), where there was an upward displacement of the westerlies for that year (Newman et al., 2016). This is thought to be due to wind and temperature structures causing planetary waves to propagate from the northern subtropics to the equatorial region, weak wind shear, and the combination of a warmer tropical troposphere but cooler stratosphere for that period. 

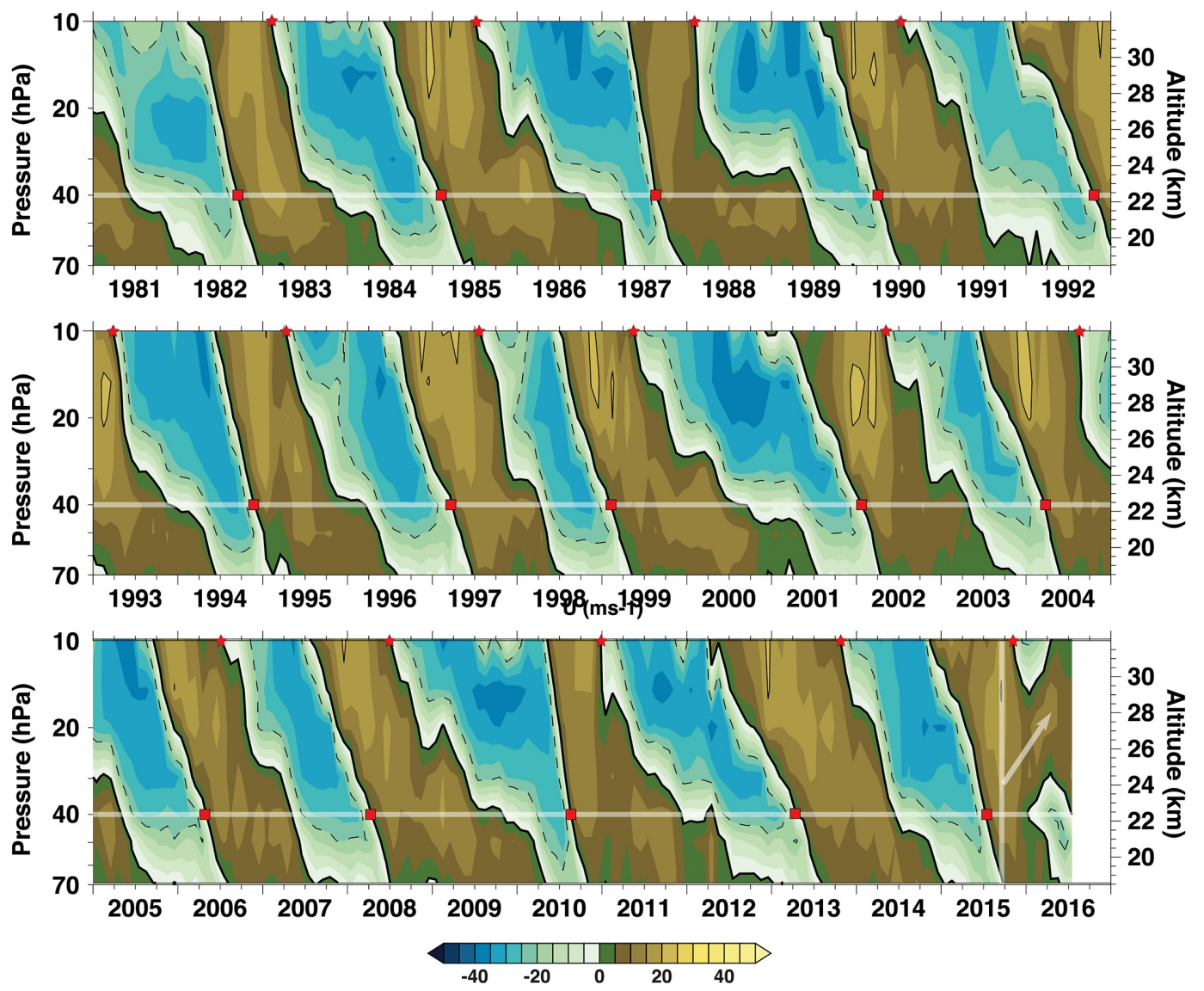

Figure 2.4: The downward propagation of the QBO as seen in monthly mean zonal equatorial wind $\left(\mathrm{ms}^{-1}\right)$ between 70 and $10 \mathrm{hPa}$. Easterlies are shown in cyan and westerlies in green/brown. The red squares show the dates of the $40 \mathrm{hPa}$ easterly to westerly transition, while the red stars show the $10 \mathrm{hPa}$ dates of the westerly to easterly transition. Note the anomalous 2016 year where winds remain westerly at levels above around $40 \mathrm{hPa}$. Source: Newman et al. (2016). 


\subsection{Southern Hemisphere circulation}

There are distinct hemispheric differences in the Earth's atmospheric circulation. In the $\mathrm{NH}$, the presence of large land-masses with extensive regions of high elevation means that the flow of westerly winds is disturbed by the orography and planetary wave generation is enhanced (Plumb, 2010). This makes for a more variable and weaker westerly circulation in the northern winter in both the troposphere and the stratosphere, compared to the winter circulation in the SH. In addition, the enhanced wave activity in the $\mathrm{NH}$ means that the wave-driven $\mathrm{BDC}$ is stronger and the ozone layer is thicker in the NH than in the $\mathrm{SH}$ (Butchart, 2014).

Absence of large high-elevation land masses in the Southern Ocean means that the westerly winds remain relatively unobstructed in the $\mathrm{SH}$, planetary wave action is therefore relatively low, and the circulation here is able to become stronger and remain more symmetrical about the pole relative to the NH (Plumb, 2010).

On Earth, vortices develop in the both the SH and NH during their respective winter seasons. Poleward advection of air masses is turned to the right in the $\mathrm{NH}$ and to the left in the $\mathrm{SH}$ due to the Coriolis force, forming the stratospheric westerly jet (Holton, 2004). The polar stratospheric vortex - also known as the stratospheric westerly jet or the polar-night jet - comprises an annulus of strong westerly winds forming a large scale circulation surrounding the pole (Schoeberl et al., 1992). Planetary wave propagation and breaking in the stratosphere leads to more effective mixing of air and a weaker vortex in the NH (Plumb, 2010).

\subsubsection{The Southern Annular Mode}

The Southern Annular Mode (SAM) is the dominant mode of climate variability in the $\mathrm{SH}$ troposphere at middle and high latitudes (Thompson and Solomon, 2002). Also known as the Antarctic Oscillation or the High Latitude Mode - the SAM describes fluctuations in the latitudinal location of the tropospheric eddy-driven jet surrounding Antarctica in the mid-latitudes over the Southern Ocean. These fluctuations are influenced by planetary waves propagating from low latitudes (Karoly et al., 1989), and have considerable influence over temperature and rainfall distributions between Antarctica and the sub-tropics (Abram et al., 2014).

The SAM is highly seasonal, being strongest in the summer when the eddy-driven tropospheric jet is at its peak (Codron, 2005). SAM indices are normally based on anomalies in fields such as sea level pressure, geopotential 


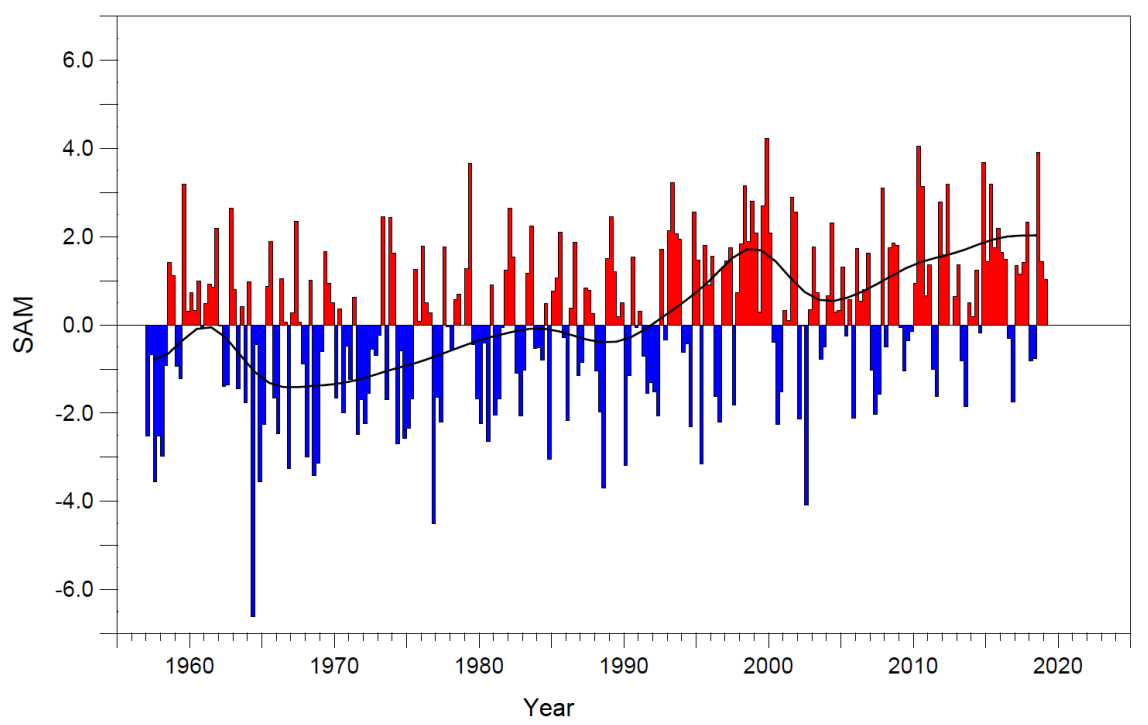

Figure 2.5: The seasonal SAM index (blue and red bars), and decadal averages (black curve) based on observations where the zonal pressure difference is calculated between latitudes of $40^{\circ} \mathrm{S}$ and $65^{\circ} \mathrm{S}$. Source: UCAR, Climate Data Guide. climatedataguide.ucar.edu/climate-data/marshall-southern-annularmode-sam-index-station-based.

height or zonal wind (Thompson and Wallace, 2000). Some indices are based on pressure differences between e.g., $40^{\circ}$ and $65^{\circ} \mathrm{S}$ (Gong and Wang, 1999), while others are calculated as the leading empirical orthogonal function of zonally varying geopotential height (Thompson and Wallace, 2000). These indices indicate the latitudinal zone at which enhanced westerly winds and greater storm activity will likely occur, and are in their positive phase when the geopotential height over the polar cap is low and the largest anomalies in the westerly winds occur closer to the pole (at latitudes of around $60^{\circ} \mathrm{S}$ ). In its negative phase, the opposite applies, where geopotential heights are greater over the polar cap, and the largest westerly wind anomalies occur further from the pole, at latitudes of around $45^{\circ} \mathrm{S}$ (Thompson and Wallace, 2000).

Over the last 30 years, a trend towards more periods of stronger positive phased SAM (Thompson and Solomon, 2002) has been observed (Figure 2.5). Recent work suggests that the SAM is currently in its most extreme positive phase over at least the last 1000 years (Abram et al., 2014). The SAM trend has been attributed to the effects of both increasing GHG concentrations and stratospheric ozone depletion (e.g., Thompson and Solomon 2002; Thompson et al. 2011; Dennison et al. 2015). This increasing positive trend in the SAM 
has long-term implications for $\mathrm{SH}$ weather and climate.

Differential warming in the troposphere as a result of higher GHG concentrations leads to changes in vertical temperature profiles, with the greatest warming expected in the highly convective middle and upper tropical troposphere (Santer et al., 2005). On the other hand, in the polar regions, high atmospheric stability limits mixing, with warming mainly confined to the surface (Goosse et al., 2018). It is likely that GHG forcing is leading to an acceleration of the SH tropospheric jet and thereby contributes to the recent positive trend in the SAM (Fyfe et al., 1999).

Ozone destruction (see section 2.2.5 below) from ODSs has been the major factor in the increasingly positive phase of the SAM over the latter part of the $20^{\text {th }}$ century (e.g., Thompson et al. 2011; McLandress et al. 2011; Ivy et al. 2017). Future changes in stratospheric forcing of surface climate in the $\mathrm{SH}$ are likely to come from both increasing GHG concentrations and from the recovery of the Antarctic ozone hole from the effect of ODSs (Butchart et al., 2000). It is thought that these two opposing factors will approximately balance during summer periods over at least the next half century (e.g., McLandress et al. 2011; Polvani et al. 2011; Perlwitz 2011), leaving the tropospheric westerly jet close to its current location.

A weakening of the stratospheric vortex in late spring and early in the summer precedes a shift toward a negative SAM index (i.e., an equatorward shift in the westerly jet) (Gerber, 2012). This signal from the stratosphere typically takes several weeks to descend to the tropopause and then descends rapidly through the troposphere over a period of just a few days (Orr et al., 2012). The opposite applies also, where a strengthening vortex results in a shift toward the SAM positive index (Limpasuvan et al., 2005). The occurrence of severe events in the stratosphere can be followed by anomalous weather at the surface that persists for several months (e.g., Baldwin et al. 2001; Thompson and Solomon 2002).

\subsubsection{The Antarctic Circumpolar Vortex}

The ACV is the defining dynamical feature of winter circulation in the $\mathrm{SH}$ stratosphere (Thompson and Solomon, 2002). The ACV extends in altitude from around $10 \mathrm{~km}$ to $30 \mathrm{~km}$ (i.e., the lower and middle stratosphere), forming early in winter each year as the stratospheric meridional temperature gradient between the mid-latitudes and the pole increases, leading to the formation of a strong westerly jet in the stratosphere, and contributing to air over

the Antarctic continent becoming much colder than the air in extra-tropical regions (Waugh and Polvani, 2010). The ACV reaches its maximum strength in late winter, and decays in spring and early summer (Waugh and Polvani, 
2010). The Antarctic Vortex Period (AVP) has been defined as the period between 19 July-1 December each year (Bodeker et al., 2005).

Wind-speeds in the ACV may exceed $100 \mathrm{~ms}^{-1}(360 \mathrm{~km} / \mathrm{h})$, which exceeds the maximum tropospheric jet-stream wind velocity and represents the highest wind-speeds of any atmospheric circulation on Earth (Schoeberl and Hartmann, 1991). The core of maximum wind-speed and PV gradients is typically located between $60^{\circ}$ and $65^{\circ} \mathrm{S}$. Areas poleward of this band of high wind-speeds also experience the lowest temperatures that occur anywhere in the world (WMO, 2015).

The vortex can be identified by meridional gradients in trace gas concentrations (Plumb and Ko, 1992) or potential vorticity (Nash et al., 1996) encircling the pole, coincident with the annulus of elevated zonal wind-speed, and strong temperature gradients. This clear signature of the ACV in the stratosphere is seen in maps displaying wind-speed, temperature, ozone mixing ratio and PV for the polar region at $70 \mathrm{hPa}$ and $530 \mathrm{~K}$ on September 16 2009 (Figure 2.6) as examples.

The meridional temperature gradient which drives the ACV is influenced by ozone and $\mathrm{CO}_{2}$ radiative forcing, planetary wave breaking and, to a much lesser extent, by the radiative effects of stratospheric sulfate aerosols (Andrews et al., 1987). As noted above, anticipated changes in the concentration of both GHGs and Antarctic stratospheric ozone are expected to lead to changes in the structure and strength of the ACV in coming decades (Butchart et al., 2000).

Because planetary wave activity in the $\mathrm{SH}$ is much lower than in the $\mathrm{NH}$, the $\mathrm{SH}$ vortex is much less affected by disruption than its $\mathrm{NH}$ counterpart (e.g., Randel and Cobb 1994). The strong zonal winds in the SH act as a wave-guide to reflect planetary waves and prevent continued poleward transport through the descending branch of the BDC equatorward of the vortex edge (Gerber, 2012). This leads to a much more symmetrical, stronger, colder and less variable vortex in the $\mathrm{SH}$, with greater isolation from the mid-latitudes (Waugh and Polvani, 2010).

\subsubsection{The ACV as a transport barrier}

It is now well understood that the ACV acts as a barrier to stratospheric transport of air masses and heat between the middle and high latitudes. However, in the late 1980s and early 1990s there was some scientific debate regarding the degree of isolation caused by the polar vortices. Some researchers argued that the vortex acts as a virtually impermeable barrier or a 'containment vessel' (e.g., Leovy et al. 1985; Juckes and McIntyre 1987; McIntyre 1989; Hartmann et al. 1989; Schoeberl et al. 1992). Others pro- 

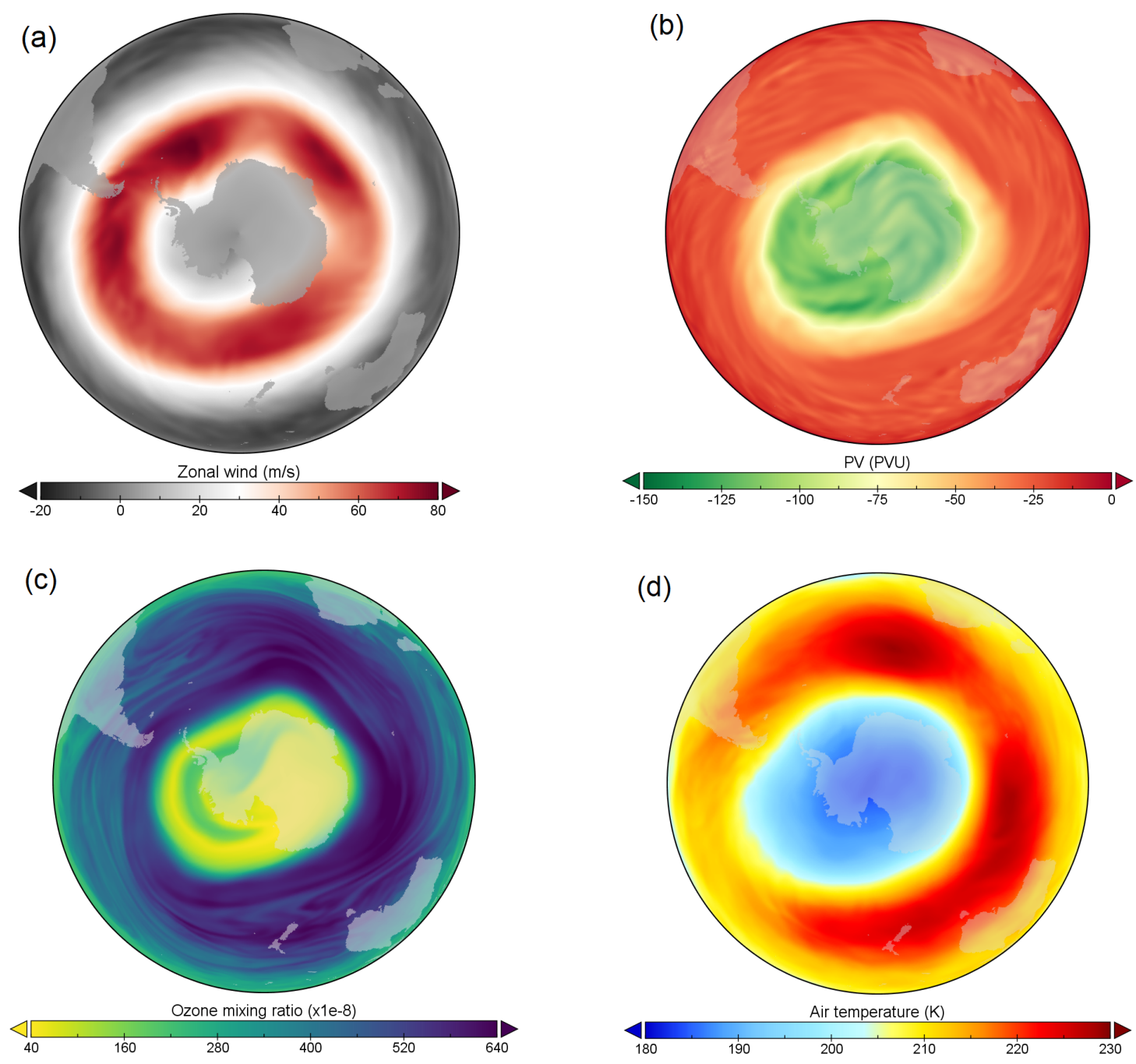

Figure 2.6: Evidence of the Antarctic circumpolar vortex in the stratosphere at an altitude of approximately $21 \mathrm{~km}$ on 16 September 2009 from (a) zonal wind-speed; (b) PV (both from ERA-Interim at 530K); (c) ozone mixing ratio; and $(\mathrm{d})$ temperature (both from MERRA2 at $50 \mathrm{hPa}$ ). 
posed that the vortex was a more permeable barrier and acts as a 'flowing processor', where air flows downward inside the vortex annulus before being dispersed equatorward in the lower stratosphere by synoptic scale waves (e.g., Proffitt et al. 1989; Tuck 1989).

Bowman (1993) found that the ACV acts as a containment vessel at most levels and during most of the vortex season, isolating the cold, dense stratospheric air above Antarctica from the warmer, less dense air at lower latitudes outside the vortex. However, the isolation of the ACV is not maintained at lower levels (below about $400 \mathrm{~K}$ ) or during the vortex breakdown period. Below about $400 \mathrm{~K}$ can be considered as the 'sub-vortex' (Haynes and Shuckburgh, 2000) where the vortex starts to disintegrate in any case. The vortex transport barrier may therefore be considered for the most part to be a containment vessel or a semi-permeable barrier (Nakamura 2008; Waugh and Polvani 2010). While warm extra-polar air can be transported along the edge of the polar vortex, it cannot easily enter it (McIntyre, 1989). Within the vortex, the cold dense stratospheric air becomes increasingly cold with neither warmer air entering from lower latitudes nor energy from the sun driving radiative heating during the polar night (Holton, 2004).

As mentioned above (section 2.2.1), the ACV shows a clear connection with surface climate by virtue of its influence on the SAM (Waugh and Polvani, 2010). The strength, size and shape of the ACV and the position and magnitude of planetary wave activity are key drivers of the magnitude and sign of the SAM (Thompson and Wallace, 2000). It is known that the ACV both responds to changes in climate, and mediates the effects of Antarctic climate change on the climate of the $\mathrm{SH}$ and beyond.

\subsubsection{ACV positive feedbacks}

There is a mutually reinforcing relationship between the presence of a polar vortex (i.e., the strong westward wind and PV gradient) and the barrier to meridional transport (i.e., the constraint on transport across the vortex) (Holton, 2004). That is, a vortex isolates the polar air and thereby strengthens the temperature gradient, as well as the concentration gradient of chemical species (such as ozone) that feed back into the dynamics of the vortex, thereby further strengthening it.

In addition, high wind shear acts to subdue wave-breaking and thus minimize irreversible mixing, which in turn helps to sustain the high PV gradients at the vortex edge - another self-reinforcing characteristic of the vortex (Holton et al. 1995; Holton 2004).

This tendency for a vortex to be self-reinforcing may be viewed as a positive feedback, where the dynamics act to amplify the contrast in the 
meridional temperature gradient, creating a stronger vortex/mixing barrier than would be expected from the temperature gradient alone.

Positive feedbacks present significant challenges to modelling efforts and represent a large component of model uncertainty (Bony et al., 2006). Webb et al. (2012) found that feedbacks in climate models can account for about twice as much of the range in climate sensitivity as differences in $\mathrm{CO}_{2}$ forcing. In climate models, feedback processes can be strongly dependent on model resolution, and any errors inherent in the model structure are amplified by feedbacks, making it difficult to ascertain the original source of the error and increasing model sensitivity to external forcings (e.g., Bony et al. 2006; Andrews et al. 2012).

Positive feedbacks experienced within the polar vortex may contribute to the difficulty that climate models have in vortex representation, particularly in effectively defining its role as a mixing barrier. Orr et al. (2012) suggest that two relevant positive feedback mechanisms may be occurring within climate models, namely weaker driving of planetary waves leading to stronger vortex winds, and the poleward shift of the tropospheric jet altering the transfer of heat and momentum in the troposphere. This suggests that an additional deceleration component may be required in models to enhance planetary waves. This 'missing' forcing may not currently be resolved in climate models, but may be due to excessive removal of eastward propagating gravity waves (Manzini et al., 2003). As noted earlier, McLandress et al. (2011) postulated that inaccuracies in the parameterization of OGWs was responsible.

\subsubsection{Ozone in the stratosphere}

Ozone has an important role as a radiatively active gas in the atmosphere, acting to heat the stratosphere, which in turn leads to the characteristic vertical profile of warming with height in this atmospheric layer (Andrews et al., 1987). The ozone layer forms in the stratosphere where maximum concentrations of ozone are located, between altitudes of $\sim 18-24 \mathrm{~km}$ ( or $\sim 30 \mathrm{hPa}$ ) (Andrews et al., 1987). The absorption of incoming solar radiation by ozone also plays an important role in preventing harmful ultraviolet (UV) radiation from the sun from penetrating to the Earth's surface, which can damage the cells of living organisms (e.g., WMO 2015).

The Chapman cycle describes ozone photo-chemical production and destruction cycles in the Earth's atmosphere (Andrews et al., 1987). Stratospheric ozone is mainly formed in the tropics, where high solar insolation drives oxygen photolysis. While ozone destruction occurs throughout the stratosphere, it is also governed by photolytic processes, and is greatest dur- 
ing spring when daylight returns at the end of the polar night (Solomon et al., 1986). Ozone destruction in the stratosphere is enhanced through the presence of industrial chemicals (i.e., ODSs) that are readily converted to active chlorine or bromine.

Containment of air within the vortex during winter and spring allows for temperatures to fall low enough (below $-78^{\circ} \mathrm{C}$ ) for the formation of Polar Stratospheric Clouds (PSCs) (Solomon et al., 1986). Reactions on the surfaces of PSCs allow for the conversion of so-called 'reservoir species' (i.e., bromine and chlorine-based chemicals present in the atmosphere such as hydrogen chloride that do not directly destroy ozone) into highly reactive chlorine monoxide $(\mathrm{ClO})$ or bromine monoxide $(\mathrm{BrO})$. When the sun returns to the polar region in spring, catalytic photolytic reactions allow the reactive chlorine and bromine to destroy large volumes of ozone. This then results in cooling of the lower stratosphere, which in turn acts to strengthen the ACV and extend the vortex season (e.g., Solomon et al. 1986; Thompson and Solomon 2002).

Figure 2.7 shows meridional cross-sections of ozone concentration (number density) in the SH atmosphere for 16 October for selected years, providing a series of snapshots of the variation in the ozone layer over time. The maximum ozone concentration is between geopotential heights of $\sim 18$ to $25 \mathrm{~km}$. Moderate concentrations of ozone (yellow) are seen to extend poleward to at least $85^{\circ} \mathrm{S}$ in 1980 (Figure $2.7 \mathrm{a}$ ), with only a small region of very low ozone values (dark blue) near the $18 \mathrm{~km}$ geopotential height, and poleward of $76^{\circ} \mathrm{S}$. By contrast, for the corresponding date in 1999, moderate ozone concentrations extend to only about $70^{\circ} \mathrm{S}$, while a larger zone of very low ozone extends to about the same latitude. For the same date in 2014, the zone of moderate ozone is seen to again extend further poleward (to around $75^{\circ} \mathrm{S}$ ), with a reduction in the extent of the zone of very low ozone (mostly poleward of $\left.76^{\circ} \mathrm{S}\right)$.

By around mid-October each year, temperatures in the Antarctic stratosphere have usually increased to the extent that PSCs are no longer present, meaning that the reactions that convert reservoir species into highly reactive oxides reduces. The reactive oxides are eventually converted back into reservoir species, and the ozone loss rate drops dramatically as the vortex begins to break up (Newman, 2010).

The ozone hole has grown over the 1980s to the early 2000s (WMO, 2015), but it has been suggested that the ozone hole has shown signs of recovery since 2000, based on a trend toward a smaller ozone hole and increases in ozone concentrations (Solomon et al., 2016). This recovery of the ozone hole may lead to weakening and warming of the ACV (Thompson and Solomon, 2002). 
An anomalously large ozone hole which was detected in 2015 is attributed to volcanic activity (Solomon et al., 2016).

Schoeberl and Hartmann (1991) found that the maximum size of the ozone hole cannot exceed the size of the ACV. While the size of the ACV changed little between the mid-1960s and 2009 (Hassler et al., 2011), average wind-speed surrounding the ACV was found to increase from 1979 to 2000 (Bodeker et al., 2002), attributed to ozone loss (Thompson et al., 2011). Increasing vortex wind-speeds are a result of steeper temperature gradients between the pole and the mid-latitudes caused by increased cooling in the polar stratosphere as a result of the lower ozone concentrations there.

\subsubsection{ACV warming}

The ACV barrier persists through to spring or early summer, gradually weakening and dissipating as the meridional temperature gradient flattens (Waugh and Polvani, 2010). This process is known as a final stratospheric warming, and takes between 10 and 40 days to progress down through the lower stratosphere (Haigh and Roscoe, 2009). Both radiative forcing (e.g., by ozone, carbon dioxide, other well-mixed GHGs and stratospheric sulfate aerosols) and dynamics (e.g., planetary and gravity wave breaking) have a significant influence on meridional temperature gradients and the dissipation of the vortex (Andrews et al., 1987).

In addition to the final warming at the end of the vortex season, a sudden stratospheric warming (SSW) can occur during the vortex season, where the winds comprising the polar vortex slow or reverse direction over the course of a few days, accompanied by a pronounced rise in stratospheric temperatures (Waugh and Polvani, 2010). SSWs are relatively regular occurrences for the $\mathrm{NH}$ vortex, but have only been recorded once - in 2002 - in the SH where planetary wave forcing is weaker (Allen et al. 2003; Thompson et al. 2005).

It has been found that SSWs are influenced by the phase of the QBO in its easterly phase the atmospheric waveguide is modified so that upwardpropagating planetary waves are focused on the polar vortex, intensifying their interaction with the mean flow and increasing the likelihood of an SSW (Holton and Tan 1980; Holton and Austin 1991).

The stronger and cooler ACV attributed to ozone depletion (Thompson et al., 2011) has also helped to prolong the vortex season. The date of the breakdown of the ACV and the final stratospheric warming has been increasingly delayed over the latter decades of the $20^{\text {th }}$ century (e.g., Butchart et al. 2000; Black and McDaniel 2007; Dennison et al. 2015), and was found to break up some 20-30 days later in the 1990s than in the 1960s (Haigh and Roscoe, 2009). CCMs have been found to overestimate this persistence of 

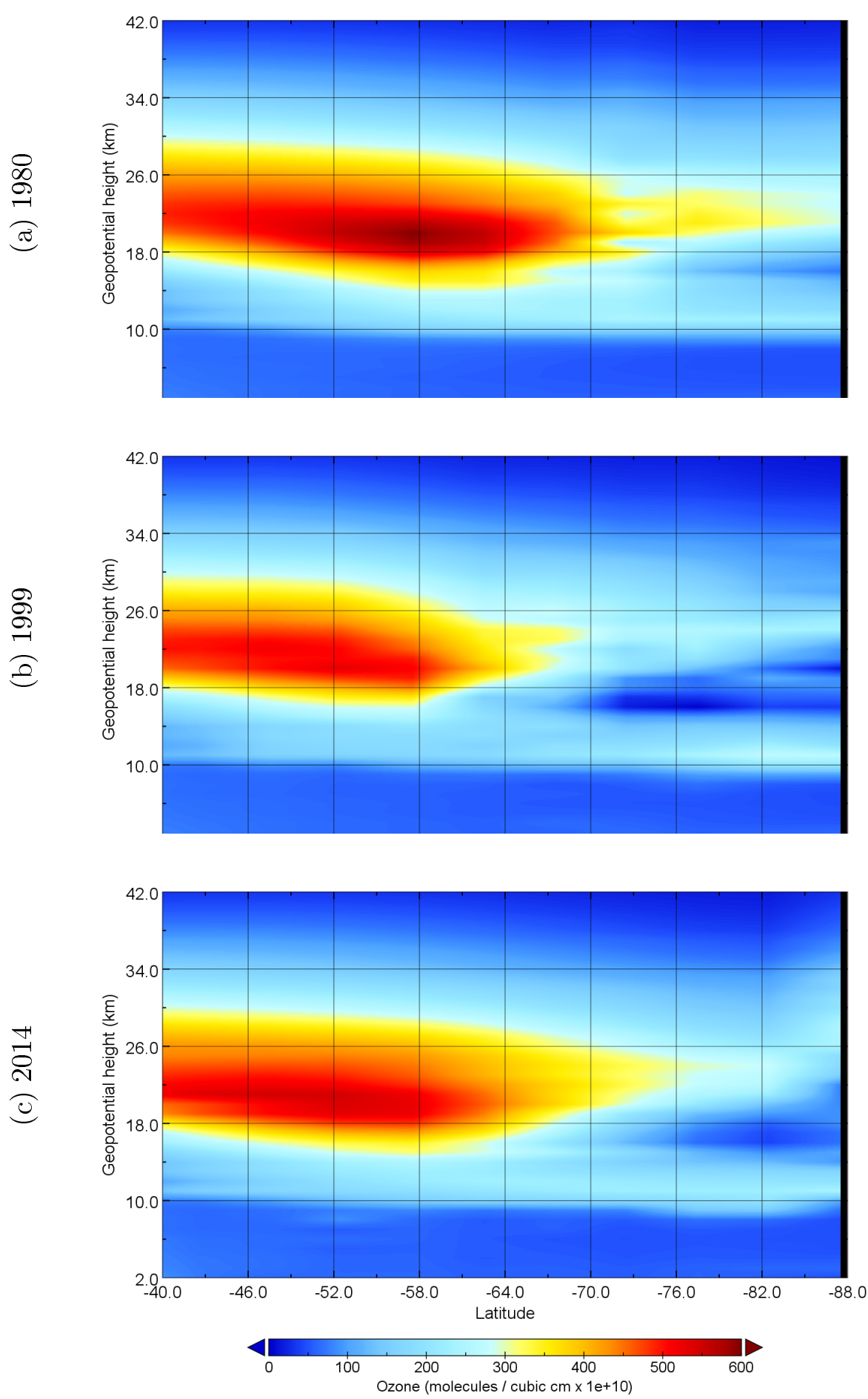

Figure 2.7: Ozone concentration for the $\mathrm{SH}$ as a function of geopotential height and latitude for October 16 for the years (a) 1980, (b) 1999, and (c) 2014. Source: Plotted from data extracted from the Bodeker Scientific vertically resolved ozone profile database (Version 1.0, Tier 0.5 ), available at: bodekerscientific.com/data/monthly-mean-global-vertically-resolved-ozone. 
the ACV, where the vortex remains intact for even longer (e.g., Eyring et al. 2006). Further extension of the vortex season is expected to occur out to the mid- $21^{\text {st }}$ century due to cooling of the stratosphere through increasing concentrations of GHGs (e.g., Butchart et al. 2000).

In the stratosphere, the net result of increasing concentrations of GHGs is a cooling effect, due to a reduction in long-wave energy transmitted upward from the troposphere and increased heat loss from the stratosphere to space (Holton, 2004). Cooling in the stratosphere alters stratospheric dynamics, including the form and behaviour of the ACV (Andrews et al., 1987). However, this effect is expected to be partially offset by the anticipated reduction in the size of the ozone hole above Antarctica (McLandress et al., 2011) as mentioned above.

\subsubsection{ACV asymmetry}

To this point, the size of the ACV has been considered in terms of the equivalent latitude, which implicitly incorporates longitudinal variation in vortex shape. However, two hypothetical vortices may have the same profile of PV gradient with equivalent latitude, but may be centred or aligned quite differently in relation to the pole, or have entirely different shapes. This is significant for two main reasons, first due to the effect that winter stratospheric changes can have on the circulation of the troposphere, which are generally related to changes in shape or location of the polar vortex (Waugh and Randel, 1999). Second, displacement of the ACV can cause regional anomalies in ozone concentrations, and hence feed back into vortex dynamics.

A comparison of the size, shape and position of the $\mathrm{NH}$ and $\mathrm{SH}$ polar vortices is illustrative, where the NH vortex is generally weaker, more variable, and less pole-centered. These differences are in turn apparent in the respective level of isolation of each vortex and thus the temperature differences and levels of ozone loss experienced in each hemisphere. Figure 2.8 provides examples of differences in ellipse shape and location on a range of isentropic levels for a selected winter month in both hemispheres (Mitchell et al., 2015). Of note is the striking difference in the level of ellipticity between hemispheres, and the greater range of movement about the pole in the NH.

In the $\mathrm{SH}$, the $\mathrm{ACV}$ moves up to $10^{\circ}$ off the pole in October, approximately along the Greenwich meridian (Grytsai et al., 2007), while in June, there is little displacement from the pole. The ACV has low interannual variability until it begins to break down in spring, and experiences little elongation (non-circularity) except during formation and breakdown when the vortex is smaller (Grytsai et al., 2007). Similar results were found using 

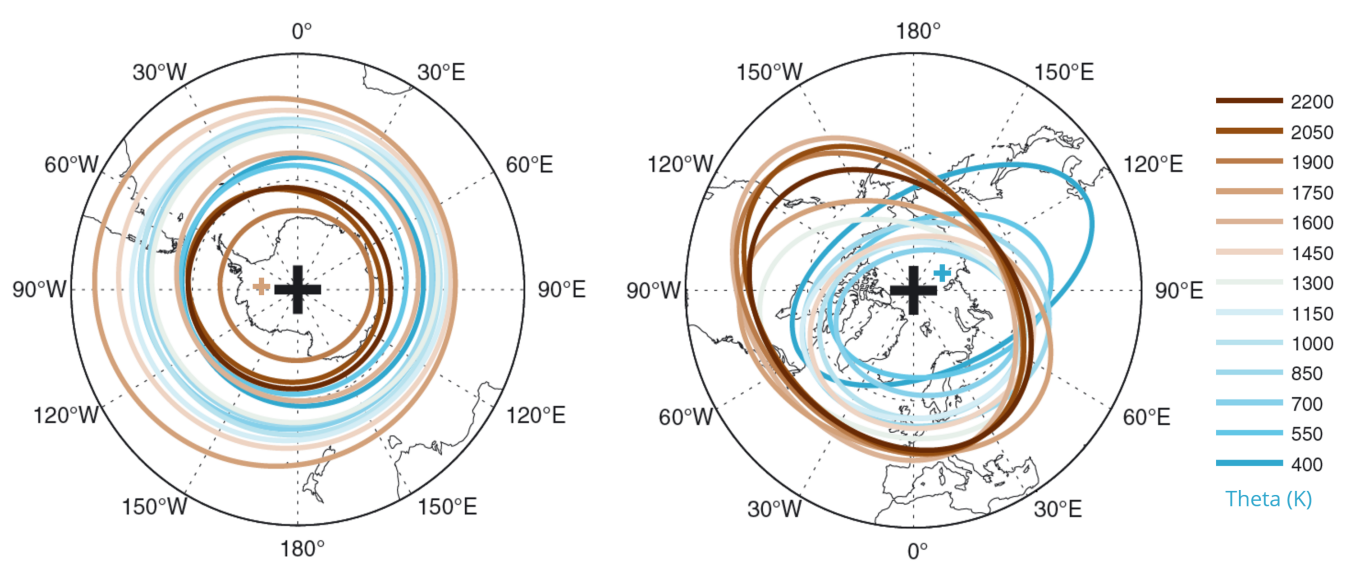

Figure 2.8: Climatological (1979-2011) vortex equivalent ellipses on a range of potential temperature surfaces in the SH in July (left) and the NH in December (right) from MERRA reanalysis. The isentropic surface is indicated by the colours displayed in the key. Each pole is shown with a black cross, while the approximate position of the centre of selected ellipses are indicated for each hemisphere $(\mathrm{NH}=400 \mathrm{~K}, \mathrm{SH}=1600 \mathrm{~K})$. Source: Adapted from Figure 9 in Mitchell et al. (2015).

principal component analysis (Huth and Canziani, 2003).

Waugh (1997) developed so-called elliptical diagnostics that describe the total area of the vortex, its orientation, elongation, and position with respect to the pole on the basis of an elliptical fit to a selected PV contour representing the vortex edge. Analysis of elliptical diagnostics for both polar vortices over a 19 year period (Waugh and Randel, 1999) showed that the location of the centre of the ACV (as represented by the centroid of a fitted ellipse) varies by about from $30^{\circ} \mathrm{W}$ to $30^{\circ} \mathrm{E}$ during late winter to spring - moving first to the west (in late August), and then to the east (in late September).

Displacement of the vortex from the pole - or lack thereof - is shown to be highly correlated with zonal wave 1 planetary wave activity (Waugh and Randel, 1999). Later studies have shown large zonal asymmetries in ozone over Antarctica during winter-spring, and attributed these to planetary wavedriven displacements of the polar vortex (e.g., Ialongo et al. 2012) caused by planetary waveforms 1 and 3 (Grytsai et al., 2017).

Waugh and Randel (1999) found that elongation of the vortex is highly correlated with zonal wave 2 activity, while Peters et al. (2015) found that enhanced wave 2 activity was critical in creating the conditions, including vortex elongation, which led to the first major SSW event in the SH in 2002. Improved understanding of the drivers of the shape, orientation, strength 
and size of the vortex, may be expected to lead to better forecasting of such SSW events.

More recently, researchers have further examined the long-term shape and location of the ACV, and found that its mean position in October (based on examination of selected Antarctic stations and their equivalent latitude) has moved to the east between the mid-1960s and 2009 (e.g., Hassler et al. 2011). These shifts are likely to be strongly inter-related to changes in ozone and dynamical transport in the southern stratospheric polar region. Lawrence and Manney (2017) applied computer vision techniques to assess the size and shape of the NH vortex, and found that these approaches were useful in characterising the vortex and identifying SSWs.

\subsection{Measurement of stratospheric state}

Stratospheric measurements have historically relied on infrastructure established to solely meet the needs of the research community. Conventional aircraft are unable to fly in the stratosphere and only a handful of experiments have been undertaken using specialist aircraft capable of flying at stratospheric altitudes (Labitzke and Van Loon, 1999).

Platforms to conduct in situ research in the stratosphere have relied on either scientific balloons carrying radiosondes or specialised stratospheric research aircraft. More recently satellite measurements have become an essential tool in stratospheric analysis, including in determining solar irradiance, upper-air winds, gas concentrations, temperature and pressure (Labitzke and Van Loon, 1999).

Measurements of air parcel transport and atmospheric state variables (temperature, pressure, density, etc.) in the stratosphere with balloons began in the 1930s (Pfotzer, 1972). The degree to which a balloon trajectory diverges over time from a trajectory calculated from reanalysis wind fields can be used to indicate that either sub-grid-scale mixing is having an influence, reanalysis winds are incorrect, or there may be regions where material stretching is occurring.

In the Concordiasi balloon campaign (Rabier et al., 2010), flights over Antarctica and the Southern Ocean provided insight into gravity waves and ozone and aerosol concentrations. Further analysis on wave-induced vertical fluctuations in Concordiasi balloon flights led to a new parameterization for simulating heating and cooling rates in the lower stratosphere (Podglajen et al., 2016).

Over Antarctica there are few regular radiosonde measurements recording stratospheric dynamical variables, making satellite measurements the main 
data source (Parrondo et al., 2006). Waugh and Polvani (2010) note that the lack of pre-satellite era stratospheric measurements in the $\mathrm{SH}$ means that analysis of trends only becomes reliable from about 1979 (i.e., from commencement of the satellite era). More recently, a wealth of additional data for the SH has been generated from the Loon project (www.loon.com), where hundreds of long-duration stratospheric balloons have been flown. Loon balloon flights provide an order of magnitude more data than all previous $\mathrm{SH}$ stratospheric scientific balloon campaigns combined. Analysis of Loon data has included comparison of measured winds with trajectories calculated from reanalyses (Friedrich et al., 2017), analysis of gravity waves (Schoeberl et al., 2017), and identifying inertial oscillations (Conway et al., 2019) in the SH mid-latitudes.

Comparison between measurements recorded in the atmosphere and output from global climate models is complicated by the fact that free-running AOGCMs can (at best) only simulate the same statistical nature of unforced variability that is the primary determinant of balloon transport. That is, the wind field on any given date from an AOGCM simulation is not expected to align with the wind field observed in reality for that day.

Overall - with the exception of the recent Loon campaign - the measurement of atmospheric dynamics in the SH stratosphere are limited. Even the balloon-based radiosonde measurements mentioned above are typically limited to measurement over land, and are also limited in elevation (up to about $30 \mathrm{~km}$ ) - leaving large measurement gaps in the $\mathrm{SH}$ and the mid to upper stratosphere (Shepherd, 2003). Boccara et al. (2008) note that the relative lack of monitoring stations or stratospheric data in the $\mathrm{SH}$, particularly south of $40^{\circ} \mathrm{S}$, means that limited data are available for assimilation, thus reducing accuracy in analyses, reanalyses, and in climate models. They find that it is therefore important to carefully assess the accuracy of such data in the stratosphere.

\subsection{Meteorological reanalyses}

Meteorological reanalysis is the method of assimilating verified scientific observations into atmospheric or Earth system models, providing a coherent spatial and temporal record of the global atmospheric circulation (Fujiwara et al., 2017). Systems in use to date typically provide global coverage, however regional reanalysis approaches are increasingly popular, allowing for assimilation of local level data, and finer spatial scaling (Whelan et al., 2018). Stratospheric research depends on temperature and wind fields obtained from reanalyses, which assimilate data from sources such as radiosondes and satel- 


\begin{tabular}{|l|l|l|}
\hline Reanalysis & Organisation & Data availability \\
\hline ERA-Interim & $\begin{array}{l}\text { European Centre for } \\
\text { Medium-Range Weather } \\
\text { Forecasts (ECMWF) }\end{array}$ & 1979 to present \\
\hline JRA-55 & $\begin{array}{l}\text { Japan Meteorological } \\
\text { Agency (JMA) }\end{array}$ & 1958 to present \\
\hline $\begin{array}{l}\text { Climate Forecast System } \\
\text { version 2: NCEP-CFSv2 }\end{array}$ & $\begin{array}{l}\text { National Centers for } \\
\text { Environmental } \\
\text { Prediction (NCEP) }\end{array}$ & 1979 to present \\
\hline $\begin{array}{l}\text { Modern Era } \\
\text { Retrospective-Analysis } \\
\text { for Research and } \\
\text { Applications: } \\
\text { MERRA/MERRA2 }\end{array}$ & $\begin{array}{l}\text { National Aeronautics } \\
\text { and Space } \\
\text { Administration (NASA) }\end{array}$ & 1980 to present \\
\hline
\end{tabular}

Table 2.2: Examples of several modern reanalysis products. Source: reanalysis.org.

lites (Parrondo et al., 2006). Reanalysis is now widely used in research in areas such as assessing the likely drivers of climate variability and change, and comparing historic and present-day climates.

Reanalysis output is generally available as website downloads, with data usually on either pressure or isentropic levels, typically for periods from 1979 onward. Data that are suitable for extraction and analysis in stratospheric research include meridional and zonal winds, pressure, temperature, water vapour, geopotential height and PV. Some reanalyses include trace gases such as ozone. A selection of some of the primary modern reanalyses are shown in Table 2.2 .

Despite their optimal combination of our best understanding of the physics and chemistry of the atmosphere, and a wide range of available observations, meteorological reanalyses can be affected by several limitations. The largescale wind field is not always accurately represented (Fujiwara et al., 2017), while mixing processes that occur at a scale smaller than the grid of the underlying Numerical Weather Prediction (NWP) model will not be adequately resolved (Boccara et al., 2008). In addition features such as the polar vortex may be displaced due to data assimilation (Stohl et al., 2004). Understanding the quality of the reanalysis fields, and awareness of possible discontinuities is therefore important for stratospheric transport and chemistry research.

The MERRA-2 and ERA-Interim reanalyses have been shown to have superior performance in the SH stratosphere when compared against NCEP- 
CFSR and MERRA reanalyses, based on a comparison against Loon balloon transport (Friedrich et al., 2017). Although MERRA-2 data were not fully available at the time of the analysis carried out in this thesis, ERA-Interim reanalysis has been assessed alongside NCEP-CFSR reanalysis. In general the ERA-Interim and NCEP-CFSR reanalyses are highly consistent between years and within each season, though ERA-Interim tends to have slightly higher wind-speeds than NCEP-CFSR. An overview of each reanalysis used in this thesis is provided below.

\subsubsection{NCEP-CFSR}

NCEP-CFSR (National Centers for Environmental Prediction-Climate Forecast System Reanalysis) is a combination of CFSR (to 2010) and the Climate Forecast System version 2, or CFSv2 (from 2011-2015), with minor changes to model parameterizations between the two versions (Saha et al., 2010). NCEP-CFSR has a latitude and longitude resolution of $0.5^{\circ}$, and 64 model levels extending from the surface to $0.266 \mathrm{hPa}$. NCEP-CFSR data is extracted at 6-hourly resolution from the NCAR research data archive ${ }^{1}$.

\subsubsection{ERA-Interim}

ERA-Interim (European Ranalysis - Interim version) is produced by the European Centre for Medium-Range Weather Forecasting (ECMWF). It has 60 vertical levels, with the model top at $0.1 \mathrm{hPa}$ (approximately $64 \mathrm{~km}$ ), and a spectral resolution of T255 (approximately $80 \mathrm{~km}$ ). For additional details refer to Dee et al. (2011). ERA-Interim uses a 4-D variational assimilation scheme to ensure that assimilation of observed climate variables is dynamically consistent. Data were extracted from the NCAR research data archive for nine potential temperature levels between $395 \mathrm{~K}$ and $850 \mathrm{~K}$ on a regular latitude-longitude grid with a horizontal resolution of $0.75^{\circ}$ and 6-hourly temporal resolution.

\subsection{Global Climate Models}

The earliest climate models began to deliver climate projections in the mid1970s, but these models only incorporated atmospheric variables (Labitzke and Van Loon, 1999). GCMs have since increased dramatically in complexity, and progressed through incorporating: land surfaces (mid-1980s); oceans and sea ice (early 1990s); sulfate aerosols (late 1990s); non-sulfate aerosols and the

\footnotetext{
${ }^{1}$ www.rda.ucar.edu
} 


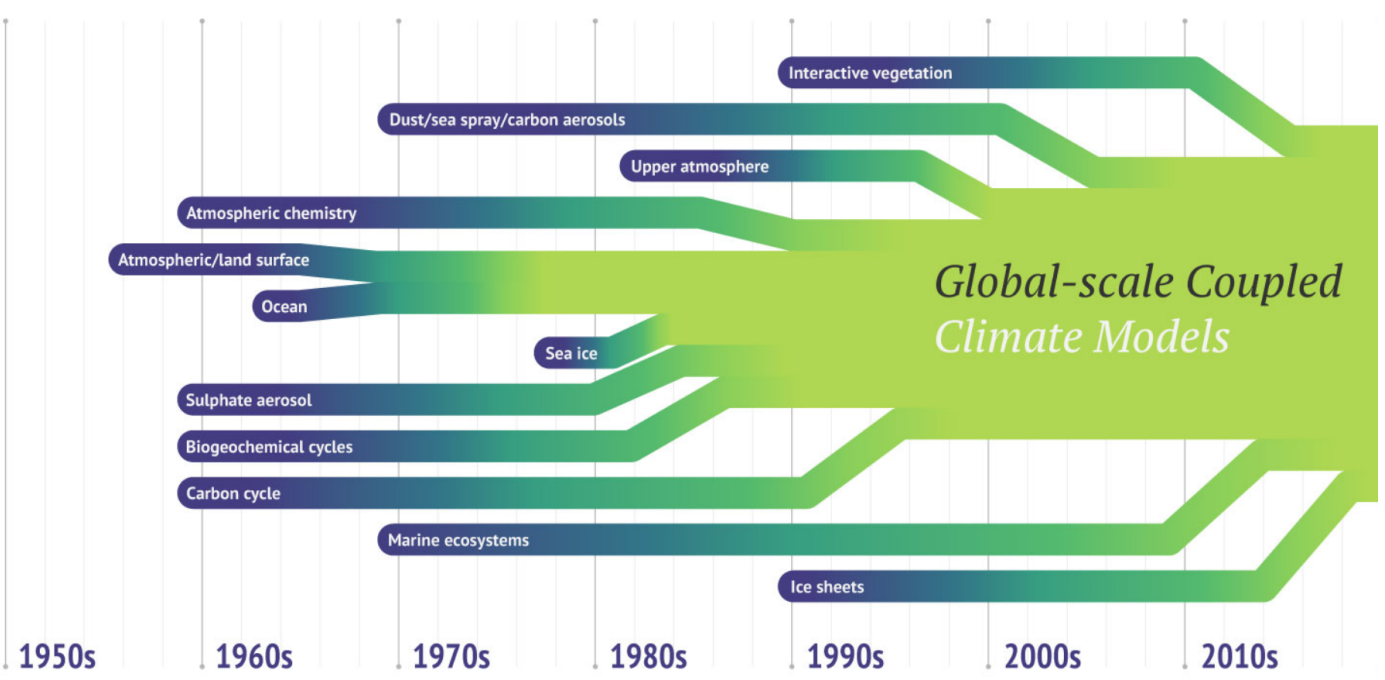

Figure 2.9: The evolution of climate model complexity. Source: Carbon Brief (carbonbrief.org/wp-content/uploads/2018/01/Evolution-ofclimate-models-final.jpg)

carbon cycle (early 2000s); atmospheric chemistry, dynamic vegetation, and land ice (2010s) (Randall et al., 2007). A schematic outlining the evolution of GCMs - now evolving into Earth System Models (ESMs) - is shown in Figure 2.9.

Climate models have improved in their horizontal resolution over this period too - from grids on the scale of over $500 \mathrm{~km}$ in the $1970 \mathrm{~s}$ to now operating on scales of around $100 \mathrm{~km}$, with the highest resolution GCMs currently running at about $25 \mathrm{~km}$ grid-scale, while regional climate models (RCMs) typically operate with spatial scales of $15 \mathrm{~km}$ or less (Randall et al., 2007). Ongoing developments are expected to see this scale continue to reduce.

Until quite recently, most weather and climate models only coarsely resolved transport in the stratosphere, and were considered sufficient for most purposes (Kidston et al., 2015). However, increased understanding of the importance of stratosphere-troposphere coupling has led to increasingly higher model tops and a more fully resolved stratosphere being used in climate models (Gerber, 2012). Strahan and Polansky (2006) found that accurate representation of stratospheric processes was possible, provided that the model incorporated a full mesosphere and high vertical resolution (i.e., around $1 \mathrm{~km}$ ) in the lower stratosphere.

It is apparent that current GCMs have shortcomings in their ability to reliably simulate the impermeability of the ACV. Being able to accurately 
represent the isolation of the ACV is a key component in simulation of realistic transport processes in the lower stratosphere, and is particularly important for enabling effective simulation of the ozone hole (SPARC, 2010). A multi-model comparison found that that while models do simulate the isolation of the ACV, its shape is unrealistically distorted from circularity, and the models struggle to simulate the orientation and equatorward tilt with height of the vortex barrier (SPARC, 2010).

Butchart et al. (2011) compared a range of climate models to assess their representation of stratospheric climate, finding that the models were typically biased toward a late breakup of the SH vortex, and displayed poorer performance in the SH compared with the NH. Waugh and Polvani (2010) note that whilst there has been a great deal of research undertaken using climate model simulations to examine ozone changes in the stratosphere, there have been few studies undertaken to investigate changes in the polar vortices, and express a need for further work on representation of the dynamics of polar vortices in global climate models. Deser et al. (2012) finds that at high latitudes the major source of model uncertainty is from internal atmospheric variability associated with the annular modes.

GCMs that include interactively coupled atmospheric chemistry are referred to as Chemistry Climate Models (CCMs). In this thesis, simulations are carried out using two reputable climate models (an AOGCM and a CCM) developed by prominent organisations in the UK and Europe. The former is the Met Office (UK) Unified Model and the latter is the SOCOLv3 CCM. Further details on these models are provided in each of the chapters which relate to the simulations undertaken using each model: Chapter 4 (UM) and Chapter 5 (SOCOLv3).

\subsubsection{Vertical interpolation}

Model output variables are transformed onto isentropic surfaces using:

$$
\Theta=T\left(p_{0} / p\right)^{\kappa}
$$

Where $\Theta=$ potential temperature, $T$ is temperature, $p_{0}$ is a standard sea level reference air pressure (typically $1000 \mathrm{hPa}$ ), $p=$ pressure, and $\kappa$ is the ratio of the gas constant to the specific heat capacity (typically $2 / 7$ is used, and known as the Poisson constant).

Values on isentropic surfaces (i.e., $\kappa$ and $\partial P V / \partial E L$ ) are scaled to a standardised reference level (in units of $s P V U \cdot \mathrm{deg}^{-1} \cdot \mathrm{ms}^{-1}$ ) of $550 \mathrm{~K}$ following the approach of Lait (1994). 


\subsection{Mixing metrics}

To better understand mixing processes and transport barriers in the stratosphere, a number of diagnostic tools have been developed by previous researchers. Chapter 3 provides details on a wide range of such metrics, and analysis is undertaken on a subset of four selected metrics. Meridional Impermeability $(\kappa)$ is chosen for the analysis outlined in Chapters 4 and 5 , as it is straightforward to calculate from both reanalysis and climate model output, without the need for complex trajectory analysis. $\kappa$ has also been shown to effectively represent the vortex mixing barrier by earlier researchers (e.g., Bodeker et al. 2002; Struthers et al. 2009). 


\section{Chapter 3}

\section{Mixing metrics in the Stratosphere}

Previous work has identified that further research to establish relationships between existing mixing metrics would be valuable (e.g., Conway et al. 2018). In this chapter, a range of metrics that have been previously developed for analysing transport barriers and diagnosing mixing in the stratosphere are reviewed and assessed.

First an overview of a wide range of methods that have been used to diagnose mixing in the stratosphere is provided. Then, an analytical comparison of a sub-set of selected metrics is undertaken with the aim of determining their relative merits, including what they each reveal about mixing and the dynamical containment of the $A C V$. Relationships are then investigated between Meridional Impermeability, Effective Diffusivity, Contour Crossing, and function $M$. While some of these metrics can be straightforwardly calculated from $P V$ fields, others require more complex trajectory analysis. This assessment provides a scientific basis for a more in-depth understanding of the transport processes that drive mixing in the Antarctic stratosphere. A paper outlining the results of the work presented in this Chapter is in preparation (Cameron et al., prep).

\subsection{Mixing metrics}

A range of diagnostic methods have been used to describe large-scale mixing in the stratosphere. Mixing on isentropic surfaces is of particular interest where there are relatively steep spatial gradients in the degree of mixing, such as across the boundaries at the edges of the sub-tropical pipe and the polar vortices (e.g., Plumb 1996; Butchart 2014).

As discussed previously (Chapter 2), the vortex edge represents a boundary where steep gradients of temperature, PV and concentrations of trace gases exist, isolating the polar stratosphere until the vortex dissipates in 
spring. Of particular interest here are how effectively mixing metrics define the vortex barrier's resistance to meridional transport, and how well they describe the relative isolation of the air within the vortex. Ideally, mixing metrics should also allow for determination of the strength and form of the polar vortex to be tracked through each season, allowing the dates of formation and final break up to be identified (Nash et al., 1996).

Both Lagrangian and Eulerian approaches have been used for diagnosing mixing (Sutton et al., 1994). Lagrangian analysis is based on a co-ordinate system that follows a moving air parcel, from the perspective of motion with the parcel. In Eulerian approaches, the analysis is based on a fixed co-ordinate system in, where the air parcel moves past fixed points. Modelling approaches can examine long-lived tracer distributions (such as the concentration of a selected chemical species) where the tracer is assumed to be advected with the flow but not influence it. Alternatively, models can examine the distribution of PV which acts as a quasi-tracer (Garny et al., 2007).

The existence of many different metrics for defining mixing and transport barriers suggests that characterization of such processes and features is challenging. Haller and Beron-Vera (2012) find that research into transport barriers has shown several shortcomings, including incorrect detection of barriers, a dependence on the frame of reference, and the use of somewhat arbitrary thresholds for their determination. A critical comparison is important for establishing if different metrics provide insight into differing features and processes, and determining the significance of such differences.

The inner (poleward) and outer (equatorward) edges of the polar vortex annulus are often defined using the maximum slope in the meridional gradient of a selected tracer, or alternatively the maximum PV gradient (e.g., Joseph and Legras 2002). The maximum in the PV gradient is where meridional air parcel exchange reaches a minimum, indicating virtual zonal flow (Joseph and Legras, 2002). This location is equivalent to the local maxima in the second derivative of the PV or tracer gradient (as shown in Figure 3.1 for a bifurcated vortex structure).

Some authors have used additional properties or applied selected thresholds to locate the vortex edges. For example, Nash et al. (1996) used the product of the maximum meridional wind-speed and maximum PV gradient; Roscoe et al. (2012) defined the inner and outer edges based on an equivalent length of 0.8, and; Serra et al. (2017) used Lagrangian Coherent Structures to define the equatorward vortex edge. Such edge definitions can be somewhat confusing, where for example, some authors define the vortex 'edge' as the entire region of high PV gradient, as opposed to a single line defining the 


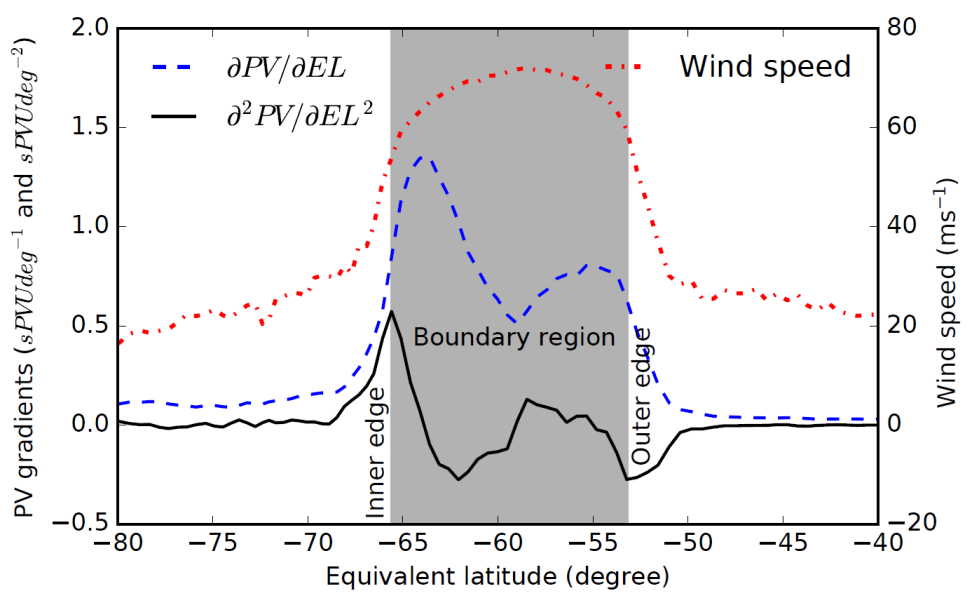

Figure 3.1: Meridional profile (by equivalent latitude) of different dynamical quantities at $530 \mathrm{~K}$ on 1 September 2007 derived from the ERA-Interim reanalysis, identifying the inner and outer vortex edges and boundary region. Source: Figure 1b in Conway et al. (2018).

edge (e.g., Sobel et al. 1997).

For the analysis carried out in this thesis, the locations of the inner and outer vortex edges have not been analysed but instead the elevated values of each mixing metric within the vortex annulus are assessed on an $\phi_{e}$ coordinate (i.e., zonal averages), with a particular focus on the peak that exists between the edges, including its magnitude and $\phi_{e}$ location, as well as Gaussian quantities such as the 'Full Width at Half Maximum', or FWHM (see section 3.3 below for details).

Some diagnostic methods rely on trajectory analysis, which involves using reanalysis wind-fields to calculate the path that air parcels have been transported. Trajectory paths can be calculated either forward or backward in time from the date of interest. Back trajectories are the basis of the Reverse Domain Filling (RDF) approach, which can simulate fields of a passive tracer at high spatial resolution, including representation of transport, filamentation and chaotic mixing. The PV value (or alternatively the tracer or chemical concentration) from the earlier date is applied at the later analysis date and location, based on the assumption that these air parcel properties are conserved for the chosen period of the calculation (e.g,. Sobel et al. 1997; Schoeberl and Newman 1995).

Early analysis undertaken for this thesis involved an initial assessment of long-duration Loon balloon data in close proximity to the ACV. RDF maps were created from NCEP-CFSR reanalyses (Figure 3.2), and compared 
with balloon trajectories for the three hours either side of the analysis time. The figure shows that balloons were typically transported zonally along the equatorward edge of the vortex, were well aligned with the vortex edges (where mixing is lowest), but were unable to cross into the vortex core.

The method used for running a trajectory model for this project is outlined in Section 3.3.1 below. An overview of a range of common mixing metrics is provided in the following sections, while a smaller subset of metrics are compared in section 3.3.

\subsubsection{Contour Crossing}

Contour Crossing (CC) involves assessing the degree of meridional transport of an air parcel away from the PV isoline in which it is initiated (Dahlberg and Bowman, 1995). CC is calculated as the change in equivalent latitude ( $\phi_{e}$ ) that an air parcel is transported (either equatorward or poleward), and thus provides a measure of the 'waviness' of the trajectory. A small range of meridional transport corresponds to flow which is closer to purely zonal and is more likely to correspond to a transport barrier.

$\mathrm{CC}$ calculations involve the use of back-trajectories, where the PV value is tagged at the initial and end points of the trajectories to evaluate transport across $\mathrm{PV}$ isolines, which may be either poleward or equatorward. Using a field of CC (i.e., a field of values in degrees of equivalent latitude that an air parcel is transported) enables the meridional profile to be calculated by zonal averaging. A threshold can be applied so that small fluctuations are not counted as significant enough to be considered as true transport across $\mathrm{PV}$ isolines, but may instead be minor perturbations that are likely to be within the error bounds of the modelling (e.g., if transport is $\leq 2^{\circ} \phi_{e}$ ).

Sobel et al. (1997) found that CC calculations using wind-fields from reanalysis are typically noisy, with low signal to noise ratios caused by variability in the underlying PV fields. This may relate to issues of data assimilation in reanalyses, where conservation laws are broken between time steps (see section 2.4 in Chapter 2). Dahlberg and Bowman (1995) found that the vortex mixing barrier was best represented when averaging $\mathrm{CC}$ values over periods of 30 days. Sobel et al. (1997) introduced the 'local gradient reversal' approach, where mixing is assessed on the basis of reversal in the PV gradient (where PV gradients are large) over a $\phi_{e}$ range of about $1-3^{\circ}$.

\subsubsection{Effective Diffusivity}

Effective Diffusivity $\left(\kappa_{e f f}\right)$ provides a measure of irreversible mixing of an advected tracer via the stretching and folding of the material contour (Naka- 


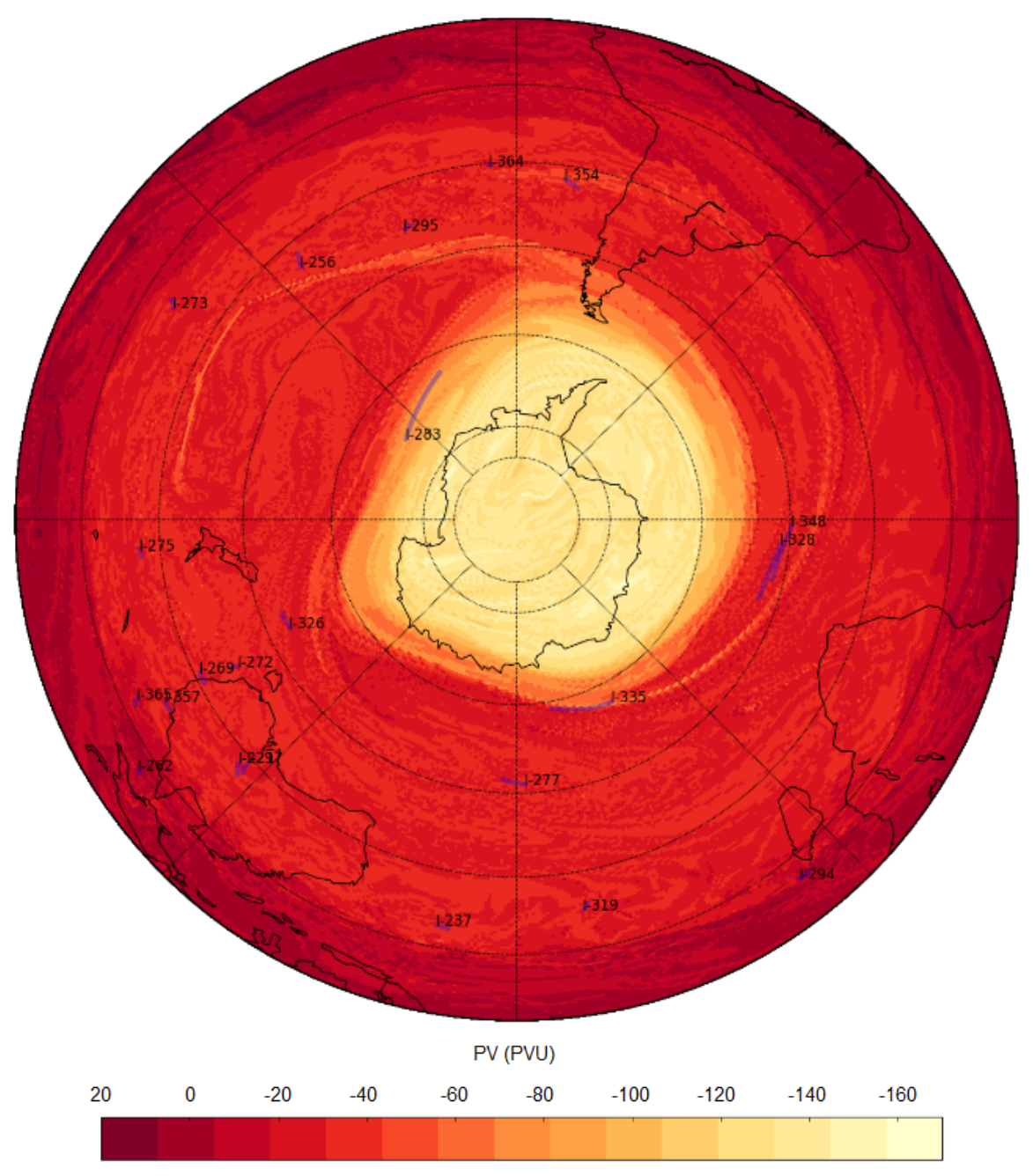

Figure 3.2: 10 day RDF plot from NCEP-CFSR for 12:00hrs on 10 September 2014 on the $550 \mathrm{~K}$ isentropic surface showing location of Loon balloons (blue lines with flight numbers) for the 3 hours either side of the RDF timestamp. The vortex mixing barrier is indicated by the orange zone of steep PV gradient. 
mura, 1996). Based on air parcel trajectory analysis, a trajectory length equal to the length of the latitude circle enclosing the same area gives $\kappa_{\text {eff }}$ of zero (i.e., purely zonal flow), thus denoting a meridional transport barrier. Higher $\kappa_{\text {eff }}$ values represent 'wavier' flow, with increasing meridional transport components. Using a trajectory model $\kappa_{e f f}$ is provided by Roscoe et al. (2012) as:

$$
\kappa_{\text {eff }}=k\left(L_{e q} / L_{\min }\right)^{2}
$$

Where $k$ is the small scale background diffusivity, $L_{e q}$ is the length of the stretched and folded tracer contour, and $L_{\text {min }}$ is the circumference of $\phi_{e}$ containing an equal area as the area bounded by $L_{e q}$. $\kappa_{e f f}$ calculations which use mass as a Lagrangian co-ordinate allow for determination of total mass flux (a measure of the instantaneous mass flow associated with divergent winds; Nakamura 1996). However, mass flux has not been widely adopted for use in mixing diagnosis. Because the small-scale background diffusivity may be unknown, and is assumed to be constant, the equation is often simplified as the dimensionless log-normalised equivalent length (EL), which is directly proportional to $\kappa_{\text {eff }}$ (Allen and Nakamura 2001; Abalos et al. 2016):

$$
E L=\ln \left(L_{e q} / L_{m i n}\right)^{2}
$$

The log-normalised EL approach means that a value of zero implies a circular tracer contour with no meridional mixing, while high mixing can be identified by relative maxima in EL (Nakamura and Ma, 1997). $\kappa_{\text {eff }}$ can also be calculated based on tracer concentration gradients (Nakamura 1996; Haynes and Shuckburgh 2000), assuming quasi-Lagrangian evolution on isentropic surfaces, and allowing for calculation without the need for a trajectory model.

$$
\kappa_{e f f}\left(\phi_{e}, t\right)=\kappa r^{2} \frac{\left\langle|\nabla c|^{2}\right\rangle}{\left(\partial C / \partial \phi_{e}\right)^{2}}
$$

Where $c$ is the tracer concentration as a function of time. $C$ represents each value of $c$ transformed to $\phi_{e}$ co-ordinates, and $r$ is the Earth's radius. The complexity of using material gradients based on trace gases led to the use of the PV field as a tracer equivalent (Haynes and Shuckburgh, 2000), which provides the EL of the PV contours (ignoring both constants from equation ??). Comparisons between EL values based on the use of a passive tracer with those undertaken using PV fields, have shown high levels of consistency between the two approaches (e.g., Abalos et al. 2016). The latter approach has been followed in the current research (i.e., using the PV field in place of air parcel trajectories). 


\subsubsection{Meridional Impermeability}

The ACV stratospheric transport barrier was diagnosed by Nash et al. (1996) in a physically meaningful way as the product of the meridional gradient of PV and zonal wind-speed. Subsequent research formalised this (e.g., Bodeker et al. 2002) as meridional impermeability (hereafter $\kappa$ ) calculated on isentropic surfaces as:

$$
\kappa_{\left(\phi_{e}\right)}=\frac{\partial P V_{\left(\phi_{e}\right)}}{\partial \phi_{e}} \cdot U_{\left(\phi_{e}\right)}
$$

Where $\partial P V / \partial \phi_{e}$ is the meridional gradient of $\mathrm{PV}$ with respect to $\phi_{e}$ and $U$ is the total zonal mean wind magnitude $\left(\sqrt{v^{2}+u^{2}}\right.$, where $v$ and $u$ are the meridional and zonal components respectively of the wind flow on an isentropic surface) averaged along the PV isoline. The units of $\kappa$ are PVU.deg ${ }^{-1} \cdot \mathrm{ms}^{-1}$. A possible limitation of the $\kappa$ metric is that it tends to conflate the two driving mechanisms, which are both functions of the windfield (i.e., a zonally wind driven vortex and a PV gradient related transport barrier associated with maximum wind-speed). The implication is that while $\kappa$ may be able to define the transport barrier, it may be less effective at defining mixing in general.

The core of the vortex annulus is marked by the steepest PV gradient and maximum wind-speed, while the inner and outer edges of the vortex annulus can be defined by the steepest rate of change in $\kappa$, calculated as the local maximum and minimum of the second derivative of $\mathrm{PV}$ with respect to $\phi_{e}$ (Nash et al., 1996).

While some authors have used only the PV gradient without multiplying by the mean zonal wind-speed (e.g., Manney and Lawrence 2016; Conway et al. 2018), this has the potential to generate areas with high spurious PV gradients outside the vortex area. This can then distort the analysis by mapping PV to locations at lower $\phi_{e}$ than they would otherwise be located. Incorporation of wind-speed in the calculation of $\kappa$ is more likely to ensure alignment with the vortex zone of high wind-speeds (e.g., Nash et al. 1996), and can distinguish a single vortex edge if multiple PV peaks exist. On the other hand, identification of bifurcation across the vortex is most apparent in the profile of PV gradient alone, while wind-speed profiles tend more toward having a single peak (Conway et al., 2018) (see Figure 3.1 above). Multiplying by wind-speed in such a case would reduce the incidence of identified bifurcation in the vortex profile. Assessment of bifurcation (see Figure 3.3) allows the region of reduced mixing to be better characterised, revealing a more complex structure that is likely to produce variable gradients in trace gas concentrations (Conway et al., 2018). Overall, for monthly averages the 


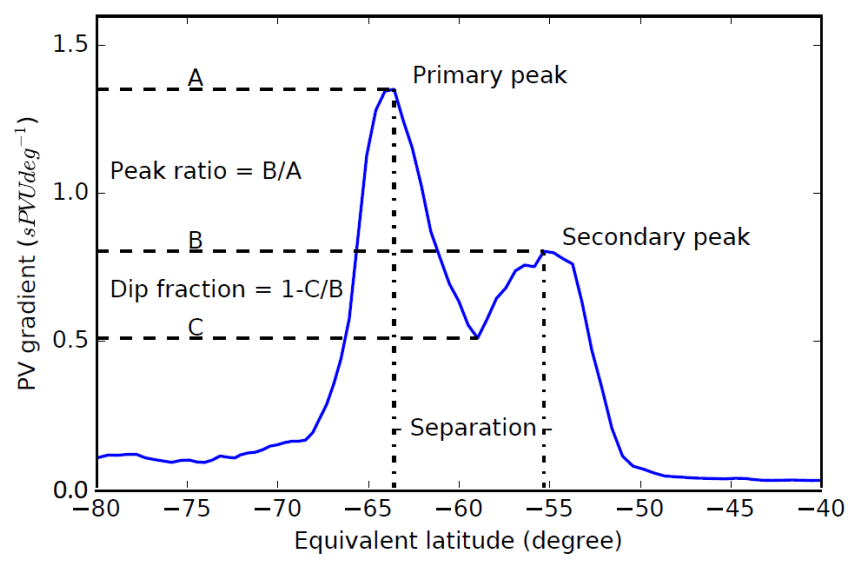

Figure 3.3: Bifurcated meridional profile of PV gradient showing peak ratio, dip fraction and separation at 530K on 1 September 2007 from ERA-Interim. Source: Figure 1a in Conway et al. (2018).

PV gradient alone is a good proxy for $\kappa$, but higher variability in both PV gradient and wind-speed on daily to weekly time periods makes multiplication of those two factors (i.e., $\kappa$ ) a more reliable metric for edge identification and analysis of cross-vortex structure.

\subsubsection{Function $M$}

Function $M$ (hereafter $M$ ) is a Lagrangian metric that describes the distance that an air parcel is advected by the background flow (Madrid and Mancho, 2009). It is calculated as the integral of the distance that an air parcel is transported over a selected time interval, using both forward and backward trajectories on either side of the chosen 'analysis time':

$$
M=\int_{t_{0}-\tau}^{t_{0}+\tau} d t \sqrt{\left(\frac{d x}{d t}\right)^{2}+\left(\frac{d y}{d t}\right)^{2}}
$$

Where $t_{0}$ is the analysis time, $\tau$ is the selected time interval, and $\frac{d x}{d t}$ and $\frac{d y}{d t}$ are the velocity fields in the zonal and meridional dimension respectively.

The vortex transport barrier is characterised by high values of $M$, associated with air parcels transported long distances where consistently high zonal winds are present. Areas away from the vortex - such as the surf zone - are characterised by eddies, wave-breaking and filaments of lower wind-speed, resulting in low values of $M$. Maps of $M$ are able to identify transport barriers, together with high definition mixing where adjacent trajectories may 

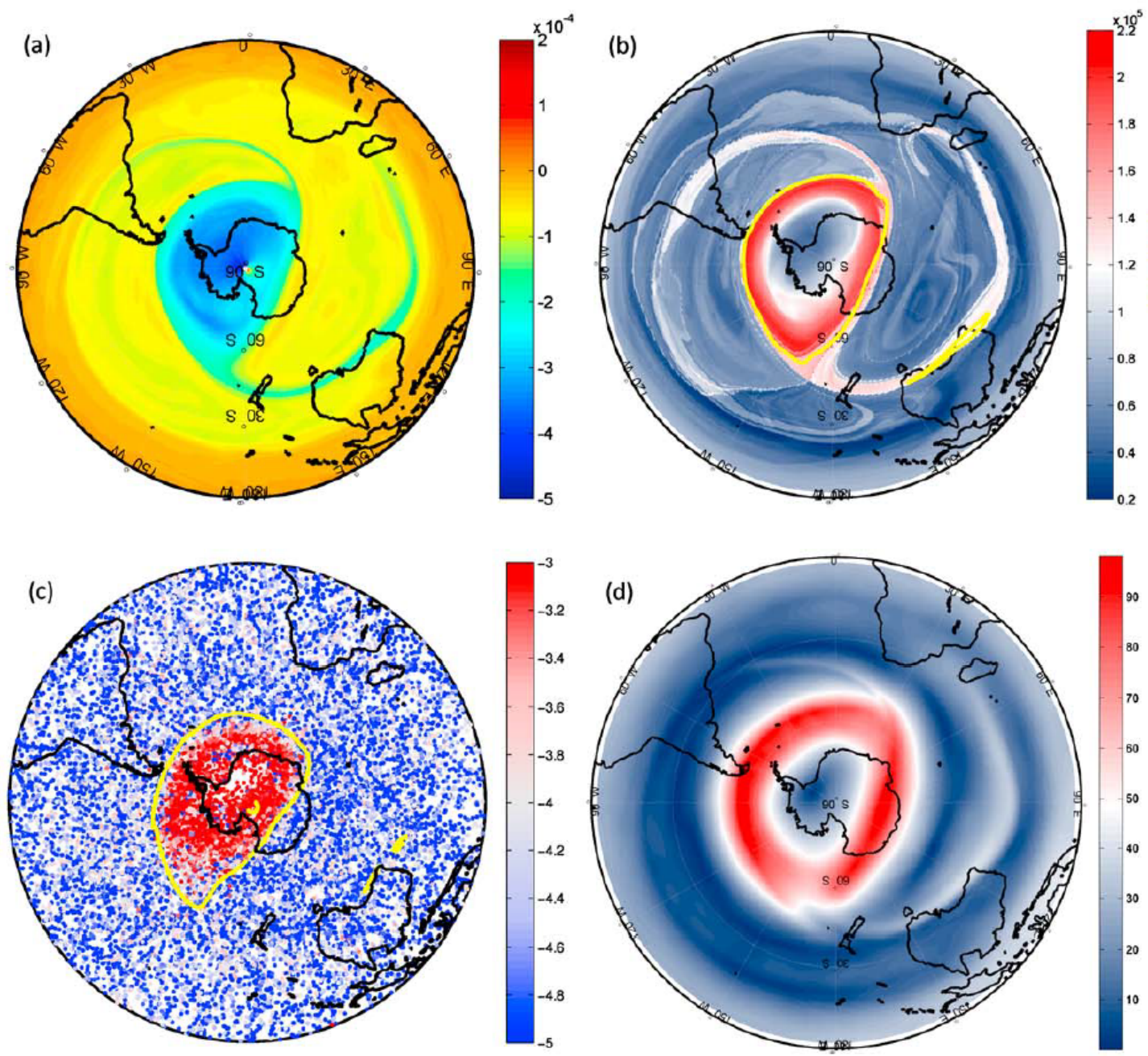

Figure 3.4: Function $M$ comparison with other mixing metrics for the 30-day period centered on 1 Oct 2009, for: (a) PV $\left(\left(K \mathrm{~m}^{2}\right) /(\mathrm{kgs})\right.$; (b) function $M$ (in $\mathrm{km})$; (c) CO tracer concentrations (logarithmic scale); (d) instantaneous wind speeds $\left(\mathrm{ms}^{-1}\right)$ at the $700 \mathrm{~K} \theta$ level. For the $\mathrm{CO}$ tracer plot, the approximate location of the vortex edge is delineated by a yellow line. Source: Smith and McDonald (2014). 
have quite distinct dynamical attributes.

$M$ effectively provides the averaged speed of an air parcel over the course of its trajectory (Smith and McDonald, 2014). A comparable Eulerian windspeed average or a field of instantaneous wind-speeds leads to a loss of information in the resulting plot, because the conservation of PV (or tracer concentration) only occurs following the air parcel (i.e., in a Lagrangian sense). This can be seen from the analysis carried out by Smith and McDonald (2014), for the 30-day period centered on 1 Oct 2009, using MERRA reanalysis (Figure 3.4). The instantaneous wind-speed field (Figure 3.4d) provides a more symmetrical, almost circular vortex, with much less detail (such as tracer filaments) compared to $M$ (Figure 3.4b). Average wind-speed over the same 30-day period (not shown) resolves even less detail compared to the instantaneous wind-speed plot. It is interesting to note that both the instantaneous PV (Figure 3.4a) and wind-speed (Figure 3.4d) maps show two filaments extending from the main vortex, but do not capture the detailed structure of those filaments or the associated stirring that is seen in $M$.

Previous studies have used $M$ to examine planetary wave-breaking and transport in the ACV region (de la Cámara et al. 2013; Guha et al. 2016), and to develop measures of the strength and area of the polar vortex (Smith and McDonald, 2014). One possible drawback of $M$ is that differences in the shape and strength of the vortex over short time-periods (e.g., days to weeks) may be missed due to $M$ typically being calculated over periods of several weeks (e.g., 10-15 days either side of the date of interest). However, the detailed mixing evidenced in maps of $M$ suggest that this is not a significant issue for transport in proximity to mixing barriers, and Smith and McDonald (2014) note that $M$ calculations over shorter time periods differ only by a scaling factor.

Manney and Lawrence (2016) note that some discussion about the usefulness of $M$ for describing flow characteristics has occurred (in particular its ability to identify LCSs - see section 3.1.7 below), but that it is effective at describing mixing, and in determining transport barriers. They also found that in the lower stratosphere the vortex mixing barrier aligns well with the maximum PV gradient and the minimum in $K_{\text {eff }}$, as well as with steep gradients in trace gases including $\mathrm{H}_{2} \mathrm{O}, \mathrm{N}_{2} \mathrm{O}, \mathrm{O}_{3}, \mathrm{HCl}, \mathrm{HNO}_{3}$, and $\mathrm{ClO}$.

\subsubsection{Contour Advection}

Contour Advection involves a similar approach to generating an RDF map (see section 3.1), but traces the rate of lengthening of entire contours by advecting air parcels at each point along the contour, as opposed to advection of individual air parcels (Sobel et al., 1997). This approach necessitates 
the addition of new analysis grid-points over the period of the simulation to keep the contour well-represented as it is mixed, stretched, and otherwise distorted. Dritschel (1989) developed the technique of contour advection with surgery, which has been used by a number of researchers (e.g., Waugh and Plumb 1994; Plumb et al. 1994). The surgery component involves removing fine scale filaments through disconnecting and reconnecting contours as required, thereby reducing the total number of particles to keep the computation manageable (Waugh and Plumb, 1994).

\subsubsection{Lyapunov Exponents}

Lyapunov exponents provide a measure of the separation of air parcels over time, under the assumption that they have an infinitesimal separation at the initial time. Computation is based on trajectory modelling, where the separation distance between air parcels after either a finite time (Finite Time Lyapunov Exponents, or FTLE; Pierrehumbert and Yang 1993), or the time taken for separation of a chosen distance/size (Finite Size Lyapunov Exponents, or FSLE; Aurell et al. 1997; Joseph and Legras 2002). It has been suggested that FSLEs in fact delineate an area of high mixing activity equatorward of the vortex core and extending into the surf zone called the 'stochastic layer' (Joseph and Legras, 2002). The stochastic layer involves fillamentation and erosion of the vortex edge, drawing away air and mixing it to lower latitudes. FTLEs (here $\lambda$ ) can be calculated as:

$$
\lambda=\frac{1}{n T} \sum_{k=1}^{n} \frac{\delta x(k T)}{\delta x\left(t_{0}\right)}
$$

Where $T$ is the time interval between resets (where one parcel is relocated to be immediately adjacent to the other), $n$ is the total number of intervals, $\delta x\left(t_{0}\right)$ is the initial distance between the two parcels and $\delta x(k T)$ is the distance between the parcels after $k$ time intervals (Bowman 1993; Garny et al. 2011; Smith and McDonald 2014). FTLEs have similarities to the calculation of $M$ above (see section 3.1.4), though $M$ makes use of both forward and backward trajectory analysis, and is not based on air parcel separation but on total length of the kinematic trajectory for individual air parcels.

The speed of air parcel separation is directly related to the efficiency of the mixing, allowing Lyapunov exponents to be used to identify areas of weak mixing associated with transport barriers. FTLEs were used by Bowman (1993) in investigations of the Antarctic polar vortex, while Garny et al. (2007) used them to assess stratospheric mixing barriers. 


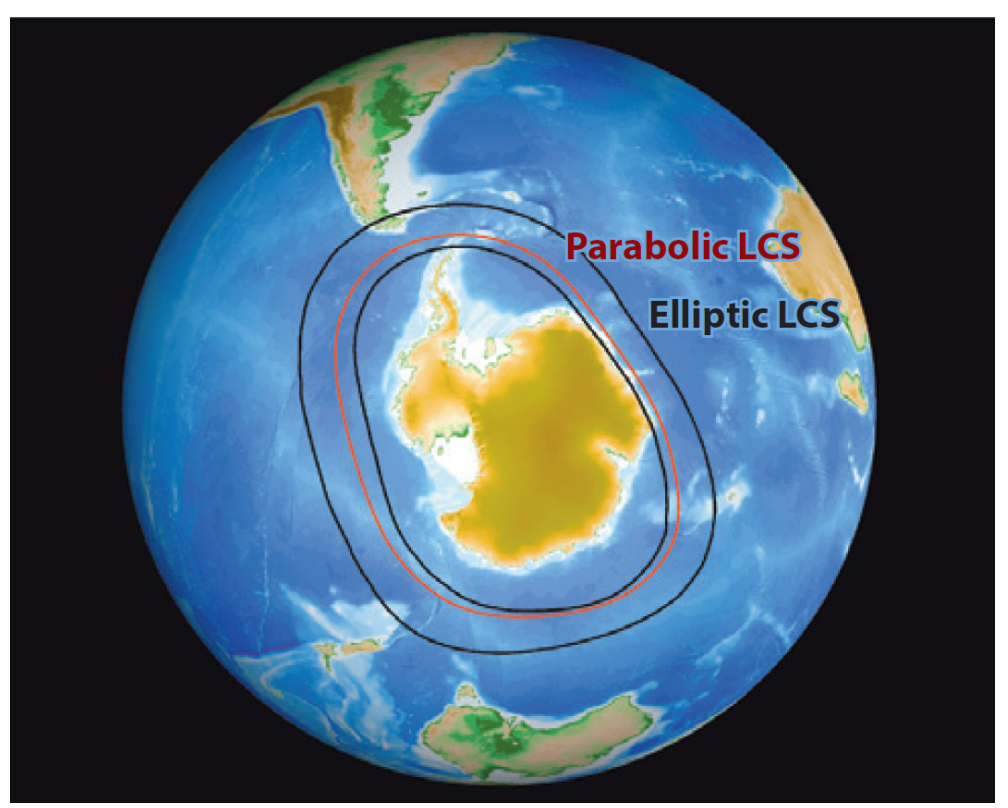

Figure 3.5: Example of parabolic (red line) and elliptic (black line) LCSs forming the core and the boundaries respectively of the zonal jet encircling the Antarctic polar vortex in the Canadian Middle Atmosphere Model. Source: Figure 9c in Haller (2015).

\subsubsection{Lagrangian Coherent Structures}

Lagrangian Coherent Structures (LCSs) use mathematical approaches to identify fluid deformation (Peacock and Haller 2013; Haller 2015) by describing the most 'influential' material surfaces that determine fluid flow. Influential material lines are those that play the dominant role in attracting and repelling neighbouring air parcels and are the LCSs of the fluid flow (Peacock and Haller, 2013). Lines where material (alternatively trace gases or PV) stretches or deforms less than its neighbours describe smooth and continuous curves of fluid advected by the flow and define transport barriers (Haller and Beron-Vera, 2012). These material surfaces are also known as strainlines or shearlines, and offer exact quantification of material transport (Haller, 2015).

In 2-dimensional flow - such as takes place on isentropic surfaces - several types of LCSs are typically assessed, including manifolds, saddle points and cat's-eyes. Manifolds are indicative of folding, stretching and filamentation in the flow and may be repelling (stable) manifolds or attracting (unstable) manifolds (Haller 2005; Haller and Beron-Vera 2012). Saddle points can be identified where stable and unstable manifolds meet, with tracer con- 
centrations at a local maximum in one direction and a local minimum in the perpendicular direction, representing a point of stagnation in the flow. Cat's-eyes identify a region where air parcels are isolated inside a bounding streamline that crosses a saddle point, while parcels outside this region are advected in opposite directions. They represent areas of anti-cyclonic eddies of high energy and instability associated with wave-breaking (McIntyre and Palmer, 1984).

Calculation of LCSs ideally requires information on the flow field at all times of interest, however, in reality information on velocity-fields is only known from observations or simulations for defined time periods (Peacock and Haller, 2013). Early LCS calculations were based on FTLEs (see section 3.1.6 above), where it was suggested that FTLE peaks coincided with LCSs. However, it was found that FTLEs were not entirely accurate at determining LCSs, and instead streamlines were found to represent truly Lagrangian transport, with no material flux across them. Peacock and Haller (2013) provide a description of the method for calculating streamlines based on an initial velocity field.

LCSs have been used to assess the polar stratospheric vortex (Figure 3.5) based on elliptic and parabolic barriers with low deformation. An elliptic barrier defines a vortex bounded by closed material lines with high shear (Serra et al., 2017), while a parabolic vortex is defined by low shear (i.e., it is bounded by high shear barriers). Recent research has shown that elliptic LCSs are able to effectively identify the edge of the Arctic polar vortex (Serra et al., 2017), based on defining the unfilamented, least disturbed and most coherent outer edge as the real edge. It has been suggested that incorrect identification of the vortex edge can occur when PV-based approaches are used - based on the inability of PV to entirely distinguish the vortex from the surf zone at fine spatial scales (Serra et al., 2017). However, LCSs are an evolving field of research that is not yet widely used in atmospheric applications. It is likely to be increasingly beneficial in analysis involving stratospheric transport barriers and mixing as computing power improves. Wide-scale application of such approaches show distinct promise, particularly in accurate vortex edge definition.

\subsection{Existing metric comparisons}

Few examples of direct comparisons between stratospheric mixing metrics are available in the literature - particularly for the SH. Relationships between different chemical tracers in the $\mathrm{NH}$ polar stratosphere were investigated by Plumb et al. (2000), with correlations analysed using canonical correlations between tracers and indicating mixing anomalies across the vortex barrier. 
Joseph and Legras (2002) compare EL with PV gradient and FSLEs, and find that the maximum EL occurs in the surf zone (at around $50^{\circ} S \phi_{e}$ ), while minimum EL corresponds to the maximum PV gradient indicating the edge of the vortex.

A comparison of daily equivalent latitude profiles of $M, \kappa_{e f f}$, and gradients in PV, $\mathrm{N}_{2} \mathrm{O}$ and $\mathrm{O}_{3}$ was undertaken by Manney and Lawrence (2016) for the NH winter of $2015 / 16$. That publication represented the most in-depth comparison of metrics undertaken to this point in time, and their plots of metrics by equivalent latitude and time of the vortex season motivated the similar assessment of metrics in the SH undertaken here. While Manney and Lawrence (2016) compared several metrics across the NH vortex season for profiles of equivalent latitude, they only undertook a direct 1:1 comparison of $M$ against PV. In this thesis 1:1 metric comparisons are taken much further, by plotting each of the metrics against one another, and against PV gradient and wind-speed, (and also PV vs $M$ ), giving a total of 15 comparisons.

In those $\mathrm{NH}$ examples, the vortex edge is characterised by the maximum $M$ values, maximum $\mathrm{PV}$ gradient, the steepest $\mathrm{N}_{2} \mathrm{O}$ gradient, and the minimum in $\kappa_{e f f}$, while the steepest $\mathrm{O}_{3}$ gradient is located about $5^{\circ} \phi_{e}$ further poleward, in-line with the fact that it originates along the inner edge of the vortex where ozone depletion is high (Manney and Lawrence, 2016). They also note that $M$ does not decline as sharply on the poleward side of the peak as either PV gradient or $\kappa_{e f f}$. This is attributed to a greater likelihood of air parcels inside the vortex annulus being advected near the inner edge, while particles outside the vortex are likely to be advected into the surf zone and thus be less likely to be transported back to near the outer vortex edge. This supports earlier analysis of CC where Dahlberg and Bowman (1995) found that reduced rates of transport were typically located on the equatorward edge of the maximum PV gradient. In addition, Manney and Lawrence (2016) also plotted $M$ directly against the PV value, finding a set of distinctive 'horseshoe' shaped curves.

\subsection{Analysis}

Analysis is undertaken here to determine how well selected metrics perform in their representation of the ACV stratospheric transport barrier. A subset of four mixing metrics is chosen for in-depth analysis and comparison in this Chapter - two that are more straightforward to calculate and two that require trajectory analysis. These are shown in Table 3.1, while Figure 3.6 provides a diagrammatic representation of the four selected metrics, indicating the method of calculation for each. 
(a) $\kappa$

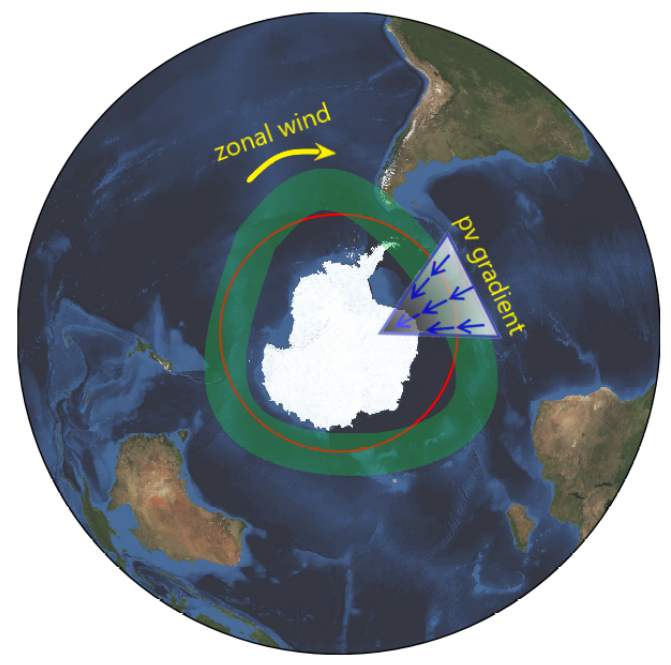

(c) $C C$

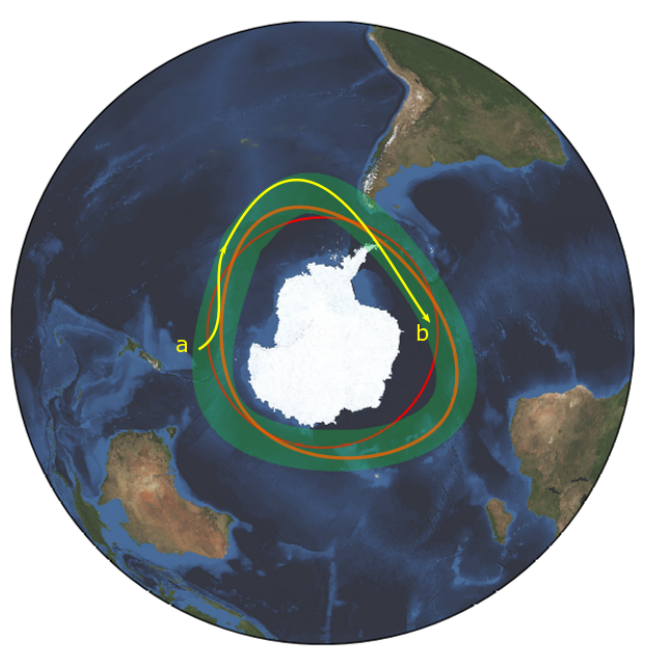

(b) $E L$

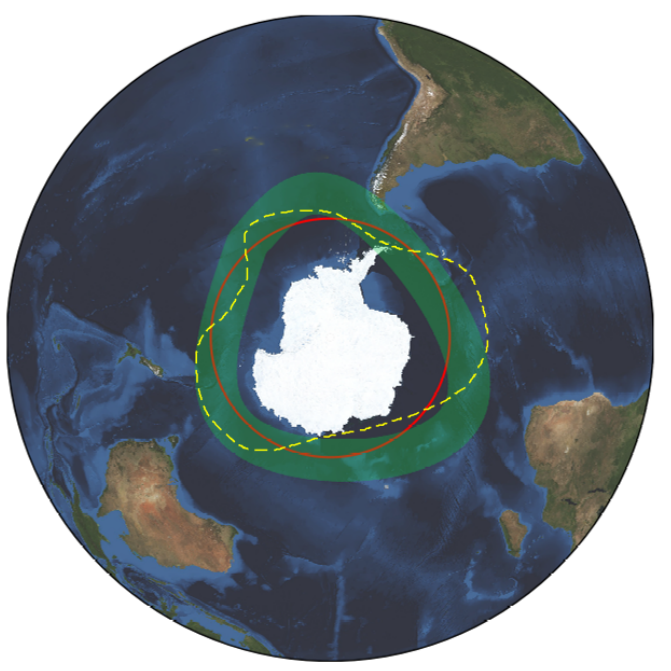

(d) $M$

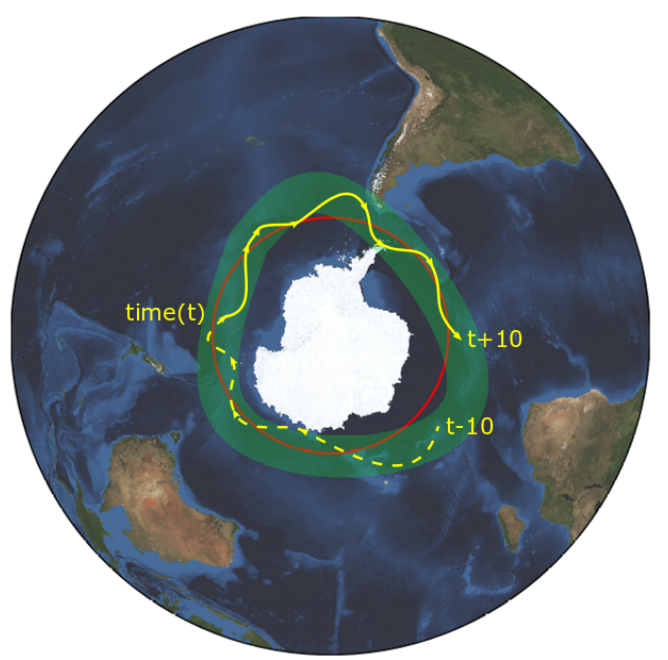

Figure 3.6: Schematic indicating calculation for each of the metrics examined in detail, showing the $60^{\circ} \mathrm{S}$ latitude circle (red) and the vortex annulus (green). (a) Meridional Impermeability $(\kappa)$ shown as the product of the zonal wind and the PV gradient; (b) Equivalent Length (EL) shown as the ratio of the PV isoline length (yellow) and the length of the latitude circle enclosing the same area as the PV isoline (in this case equivalent to the $60^{\circ} \mathrm{S}$ latitude circle); (c) Contour Crossing (CC) is shown as the change in $\phi_{e}$ that an air parcel is transported along a trajectory (yellow) between a start point (a) and end point (b) and here crosses a PV contour (orange) that corresponds to $60^{\circ} \mathrm{S} \phi_{e}$, experiencing a net poleward transport of about $2^{\circ} \phi_{e}$; (d) Function $M(M)$, shown as the total distance travelled by an air parcel along a trajectory in the chosen period either side of the analysis date (in this case 10 days). 


\begin{tabular}{|l|l|}
\hline Metric & Key References \\
\hline Contour Crossing (CC) & $\begin{array}{l}\text { Dahlberg and Bowman 1995; So- } \\
\text { bel } \text { et al. 1997 }\end{array}$ \\
\hline $\begin{array}{l}\text { Effective Diffusivity }\left(\kappa_{e f f}\right) \\
\text { Equivalent Length }(\mathrm{EL})\end{array}$ & $\begin{array}{l}\text { Nakamura 1996; } \\
\text { Haynes and Shuckburgh 2000 }\end{array}$ \\
\hline Meridional Impermeability $(\kappa)$ & $\begin{array}{l}\text { Nash et al. 1996; Bodeker et al. } \\
2002\end{array}$ \\
\hline Function $M(M)$ & $\begin{array}{l}\text { Madrid and Mancho 2009; Smith } \\
\text { and McDonald 2014 }\end{array}$ \\
\hline
\end{tabular}

Table 3.1: Metrics analysed in this chapter, showing key references.

Given the computational cost of running a trajectory model (both forward and backward for 10 day periods) to calculate $M$ and CC, simulations were only carried out for five months during winter and spring (July to November) for three selected years on the $550 \mathrm{~K}, 650 \mathrm{~K}$ and $850 \mathrm{~K}$ potential temperature surfaces. Over the course of those months, the trajectory model was run for periods 10 days apart (again to reduce the computational burden). See section 3.3.1 below for details on the trajectory model. Daily data were available for the metrics that did not require the use of a trajectory model (i.e., $\kappa$ and EL).

Metrics are calculated from output fields from NCEP-CFSR and ERAInterim reanalyses for the three selected years: 1980, 1999, and 2014. These years relate to the period early in the development of the ozone hole, the time around the maximum size of the ozone hole (discounting maxima of volcanic origin), and a relatively recent year respectively. The $550 \mathrm{~K}$ level is the focus of this chapter as it corresponds well with peak concentrations of ozone in the Antarctic stratosphere. Results from other levels are presented in the Appendix, and are also discussed below. For each day, the value of the metric is shown for all equivalent latitudes from $50-70^{\circ} \mathrm{S}$, in $0.5^{\circ}$ increments.

The $\kappa_{\text {eff }}$ used here is based on EL from Equation 3.3 - with the two terms used interchangeably here. For calculation of contour-crossing, a 10 day back-trajectory was used, based on a threshold for transport across PV contours of $2^{\circ} \phi_{e}$. The standard deviation of the change in $\phi_{e}$ (in degrees) over the 10 day period is calculated in preference to the actual $\phi_{e}$ change. It was found that high variance in the rate of CC corresponds to more 'waviness', while a change in only the value of $\phi_{e}$ over time may correspond to gradual change over the chosen period without high variance.

A sample comparison of meridional profiles for the four metrics is shown in Figure 3.7, averaged for September 2014 in the appropriate units of each 
metric. Here, $M$ uses a 30 day trajectory (i.e., for 15 days either side of each day in September 2014) in contrast to the 10 day periods used elsewhere. A 10 day $M$ profile was also run (not shown), and was found to have a flatter curve, with lower peak values of $M$ - but consistent with previous work these differ only by a scaling factor (Smith and McDonald, 2014). Both time periods for $M$ were found to produce similar smooth curves, suggesting that both the zonal averaging and the averaging over the month results in loss of definition using $M$.

The vortex edges may be defined by the steepest gradient of the curve (by $\phi_{e}$ ) for $\kappa$ and $M$, and by values tending toward zero for EL and CC. The location of the equatorward vortex edge (all in $\phi_{e}$ unless noted otherwise) compares well between EL, $\kappa$ and $M$ (at approximately $55^{\circ} \mathrm{S}$ ), while for CC in this example the equatorward edge is located further South (at about $59^{\circ} \mathrm{S}$ ). The poleward vortex edge is fairly well aligned for $\kappa$, EL and CC (at about $67^{\circ} \mathrm{S}$ ). For $M$, the poleward vortex edge is located further South (at about $70^{\circ} \mathrm{S}$ ), consistent with results found for $M$ in the NH (Manney and Lawrence, 2016) attributed to air trapped inside the vortex being more likely to be transported close to the vortex edge, than air outside the vortex, which may be transported away into the surf zone where it is further mixed.

Metrics were compared across the three selected years using daily data calculated every 10 days, and based on Gaussian quantities: FWHM (as an indication of the annulus width), and the normalised maximum value (indicating the peak strength for each day - normalised so that typical values fall between 0-1 based on the full range of values for each metric over the three selected years, allowing for ease of comparison between metrics); the latitude of maximum value (indicating the vortex peak location); and, the date of the maximum value (to examine how the metrics varied in their representation of the timing of peak vortex strength). The degree of uncertainty in the metrics was calculated by examining the variability between the 3 chosen years. Although this is a very coarse assessment approach for uncertainty, it was felt that it was the most appropriate method given the limited years of available data.

\subsubsection{Trajectory model}

Both $M$ and CC require kinematic particle trajectories to be calculated from reanalysis. The trajectory model used in this study uses a standard fourthorder Runge-Kutta integration scheme with a 1-hour time-step. The 6-hourly reanalysis wind-speeds are determined for the trajectory position using linear interpolation in time and space. Caution must be taken with this approach, as it has been found that discontinuities in the reanalysis fields can lead to 


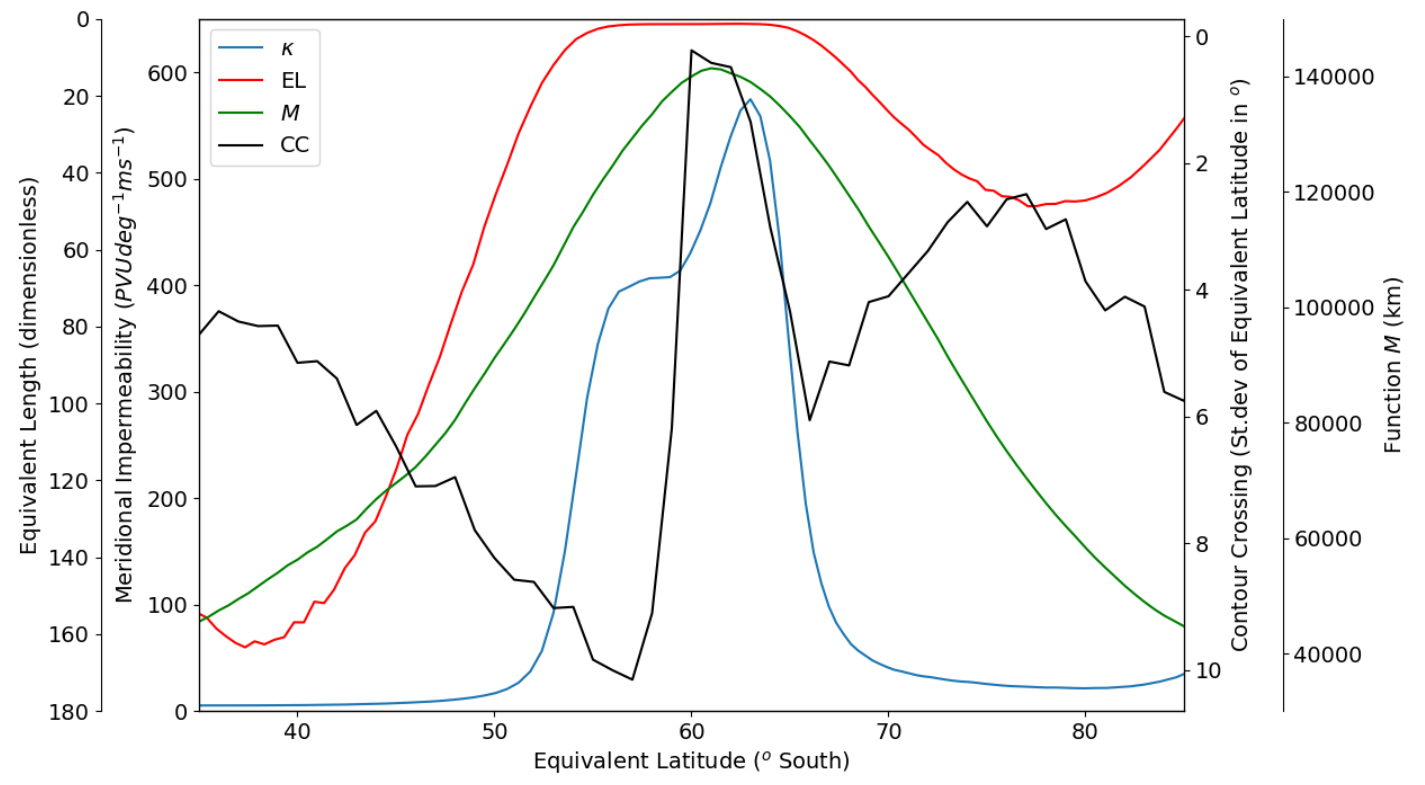

Figure 3.7: Monthly average profiles of $\kappa$ (blue), EL (red), $M$ (green), and CC (black), by equivalent latitude. Data is from NCEP-CFSR for September 2014 at $550 \mathrm{~K}$. 
inconsistencies in the calculation of atmospheric dynamics based on trajectory analysis. Such discontinuities are thought to be due to data assimilation in the reanalysis (as noted in Chapter 2), and can be reduced through the use of cubic spline interpolation (Friedrich et al., 2017). Because linear interpolation is used here, it is accepted that there may be some non-physical outliers that may affect the veracity of the trajectory results.

A quasi-uniform-area grid with a nominal resolution of $1^{\circ}$ latitude is used here to initiate the trajectories. The uniform-area grid has the advantage over a regularly-spaced latitude-longitude grid of not having the spatial resolution increasing towards the pole, and thus avoids bias in calculations that are scale-dependent. In addition, a polar stereographic grid is used south of $70^{\circ} \mathrm{S}$ to avoid the singularity at the pole.

The hourly PV values along the trajectories were assigned using a postfill method, where PV values are taken from the trajectory's location at the previous and next 'known' time (i.e. at the 6-hourly reanalysis times). The hourly PV values are then interpolated between the two known PV values. This scheme avoids interpolation errors that arise when using spatio-temporal interpolation due to the PV field being advected with the wind. The initial and final PV values are simply interpolated from the reanalysis.

\subsection{Results}

\subsubsection{Meridional profiles}

First, to explore the internal variability of the data across the chosen years, three-year averages and standard deviations for each metric were calculated. This allows for a coarse initial determination of how effective each metric is at defining the position, width, and strength of the ACV over multiple years. Figure 3.8 shows the 3 -year mean and 1 standard deviation either side of the mean for daily values taken every 10 days over the period 1 July to 30 November (from NCEP-CFSR reanalysis), with each metric profile by equivalent latitude at $550 \mathrm{~K}$. The units for each metric in Figure 3.8 and for the Figures that follow are: $\kappa=P V U d e g^{-1} m^{-1}$ (hereafter $\kappa \mathrm{U}$ ), $\mathrm{CC}=\phi_{e}$, $M=k m, \mathrm{EL}=$ dimensionless, $\mathrm{PV}$ gradient $=P V U d e g^{-1}$, wind-speed $=$ $m s^{-1}$.

Figure 3.8 indicates that there are distinct vortex profiles associated with different times of the season for each metric. In particular, each metric shows a more equatorward and wider vortex barrier in July, with a steeper, narrower and increasingly more poleward barrier near the end of the season. The significance of such differences is indicated by the shaded areas that do 

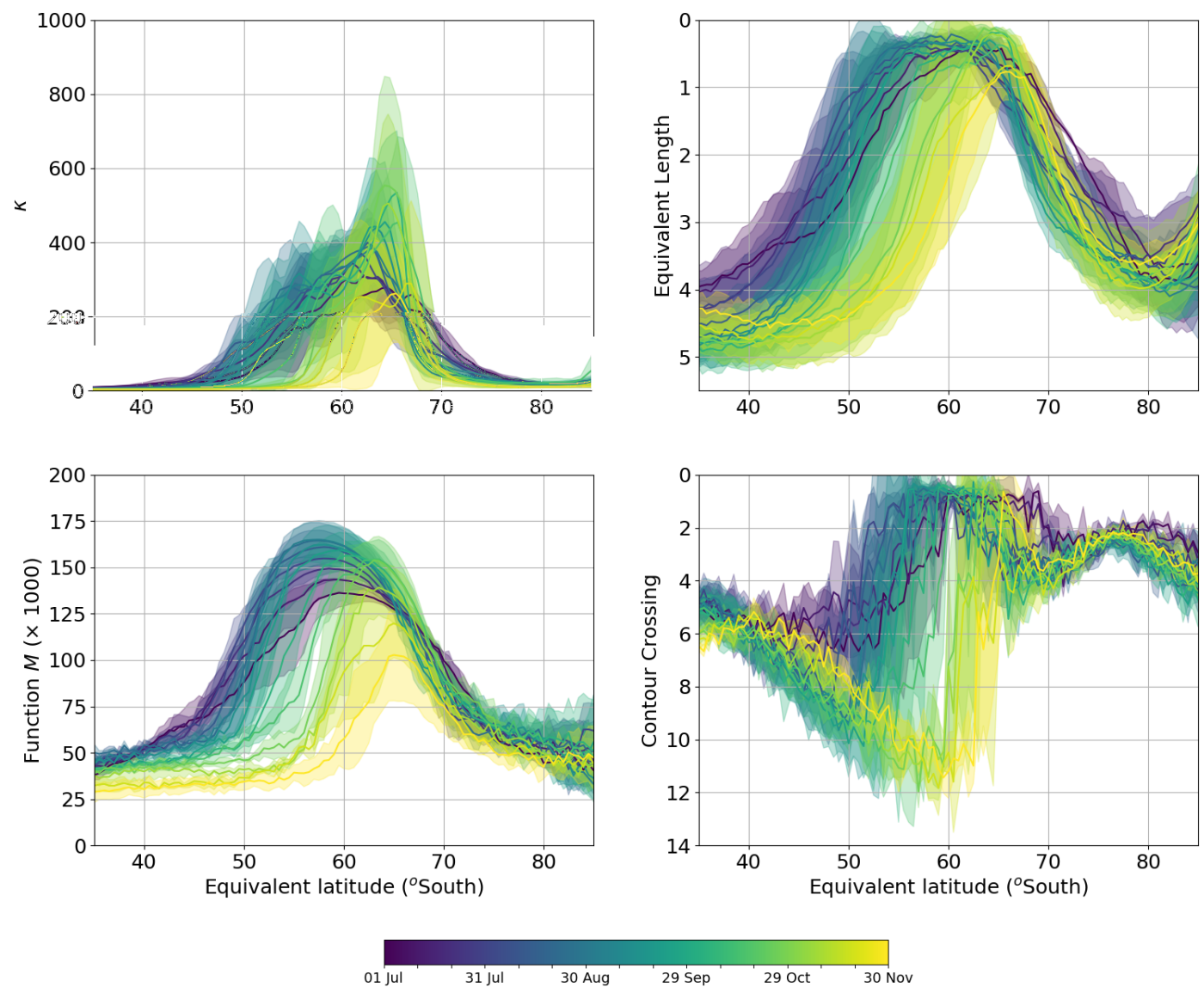

Figure 3.8: Mixing diagnostics at the $550 \mathrm{~K}$ level showing the mean (coloured lines) and 1 standard deviation either side of the mean (coloured shading) averaged for the three selected years $(1980,1999,2014)$. Daily values are shown, every 10 days, and are from NCEP-CFSR reanalysis for the period from 1 July-30 November. Metrics shown are $\kappa$ (top left), EL (top right), $M$ (bottom left), and CC (bottom right). 
not overlap. The high degree of overlap of the uncertainty bands in each metric poleward of approximately $70^{\circ} \mathrm{S}$ indicates that little variation occurs inside the vortex annulus across the season, and the main zones of interest are therefore the vortex barrier itself $\left(\sim 50-70^{\circ} \mathrm{S}\right)$ and the equatorward vortex edge and surf zone $\left(\sim 40-50^{\circ} \mathrm{S}\right)$.

\section{Annual and seasonal variation}

The following section examines the behaviour of each of the four metrics between the three selected years, and across the vortex season. Care must be taken here not to interpret the results from these three chosen years as indicative of a trend, as the selection of individual years risks choosing outliers that may not be representative of any patterns that could be detected over multiple years (e.g, over a $\sim 30$ year data period). Figure 3.9 provides equivalent latitude profiles for each of the selected years and metrics, corresponding to the plots averaged over all years seen above (Figure 3.8).

The peaks for both $\kappa$ and $M$ are higher in the later years, corresponding with a narrowing and steepening in the profiles for both metrics. This is consistent with the zones of low EL and low CC, which also narrow through the season, particularly in 2014. There are also some extremely sharp peaks in both $\kappa$ and $M$ in October in 2014, indicating a strong and narrow vortex barrier at that time.

Based on these profiles, CC appears to be least effective at defining the vortex structure when compared with the other metrics. For CC there are many values at or close to zero, which are not able to define detailed internal structure of the vortex, but rather indicate only the width of the vortex barrier. While peak values in $M$ roughly coincide with those of $\kappa$, there is little cross-vortex detail in $M$ compared with $\kappa$. Indeed, $\kappa$ regularly exhibits a bifurcated profile, also observed to some degree in EL. This is a feature that is seen in the early to mid-season period, rather than late in the season - narrowing of the barrier toward the later stages of its existence appears to limit it to a single peak at that time.

Bifurcation results from 'kinks' in the PV gradient across the vortex, and is most apparent in $\kappa$ due to its direct reliance on PV gradient in its calculation. Two $\kappa$ peaks, with lower values in between, correspond well with two 'peaks' of low EL, with higher values between - indicating wavier flow and a weaker mixing barrier within the vortex annulus at those equivalent latitudes. It is interesting to note that this internal mixing structure of the vortex is also identified by the parabolic LCS where some deformation occurs (as shown in Figure 3.5). The parabolic LCS is characterised by a zone of low shear bounded by high shear barriers (and is otherwise known as an 
1980
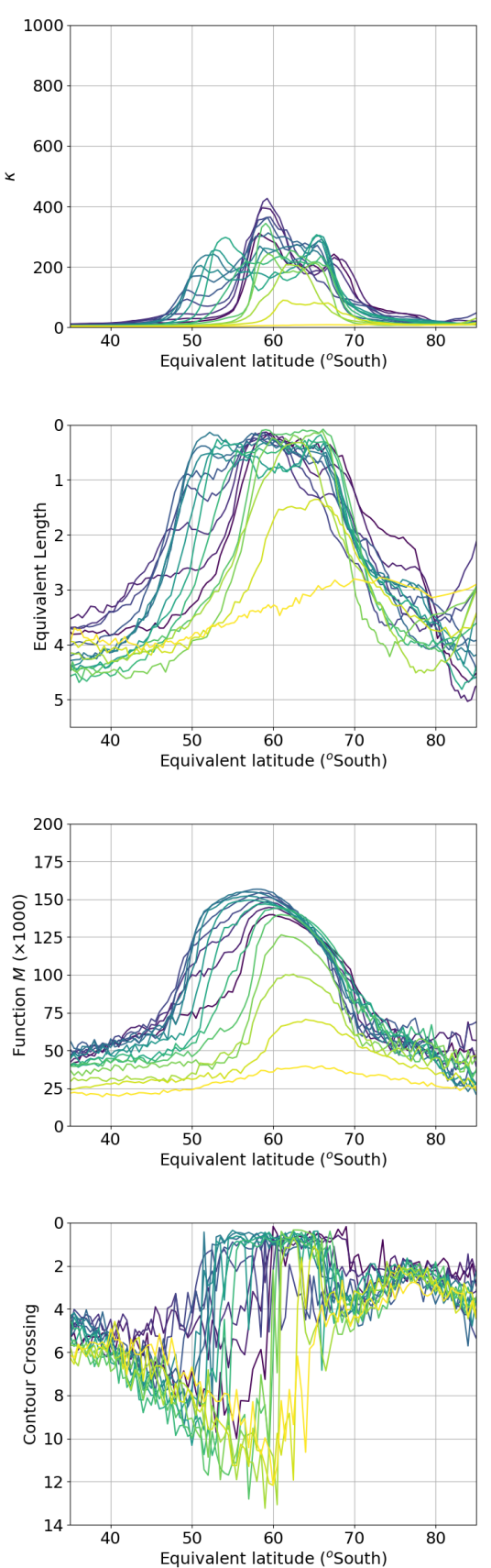

1999
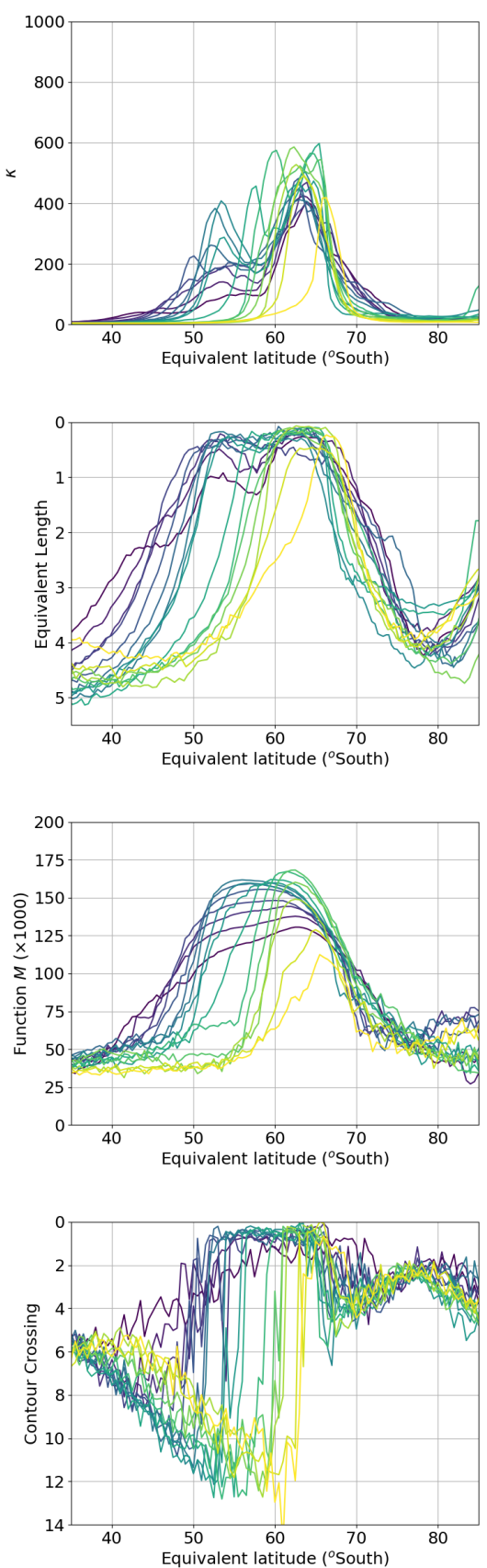

2014
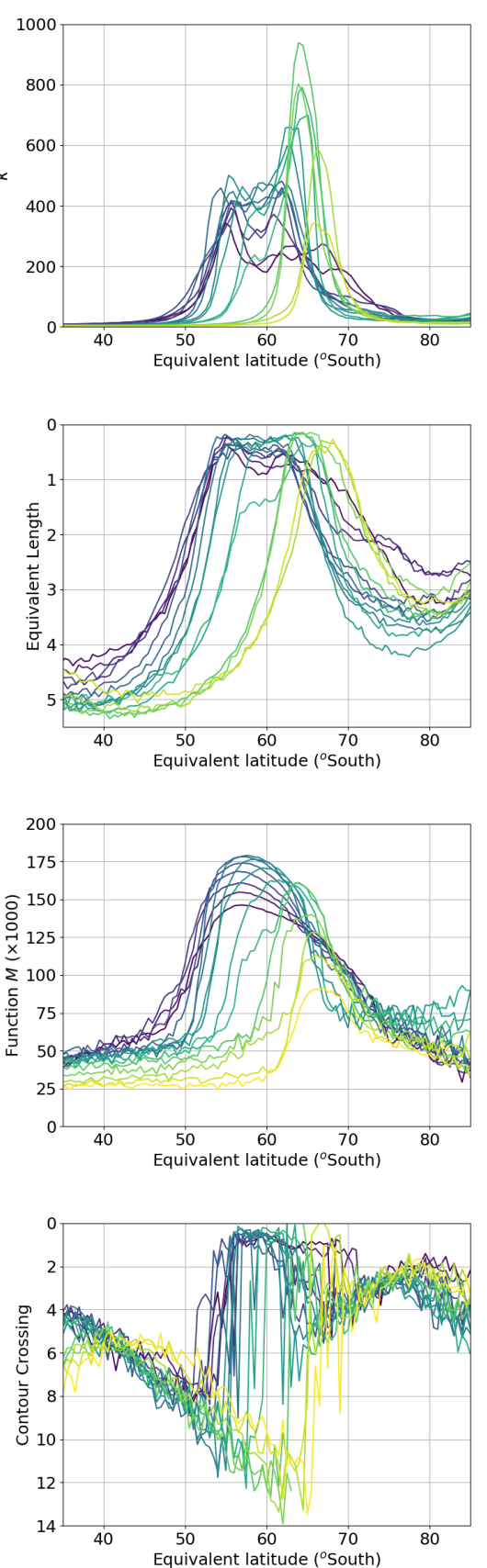

Jul

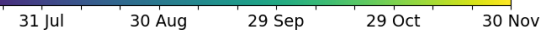

Figure 3.9: Comparison of mixing diagnostics at the $550 \mathrm{~K}$ level, calculated using daily averaged values taken every 10 days from NCEP-CFSR reanalysis for the period from 1 July-30 November (colour scale) for 1980 (left), 1999 (middle), and 2014 (right). Metrics shown are $\kappa$ (top), EL (2nd row), $M$ (3rd row), and CC (bottom). 
unsteady 'generalised jet core' - Serra et al. 2017). Exploration of LCSs has resulted in the development of computational processes to detect the presence of parabolic barriers (Farazmand et al., 2014).

The loss of cross-vortex detail in $M$ and CC is explained by the fact that these metrics rely on multi-day trajectories, where most air within the vortex annulus is transported over long distances with low rates of PV contour crossing, leading to less differentiation across that region (and an apparent wider barrier also). These results begin to provide some insight into the varying aspects of mixing and transport that can be determined from the different metrics. Internal vortex structure is better determined using $\kappa$ or EL to indicate the double-walled structure which has only recently been described in detail (Conway et al., 2018), and is shown to be common in the ACV in September at levels between $475 \mathrm{~K}$ and $600 \mathrm{~K}$. This feature warrants further research to better understand its dynamical implications based on either $\kappa$ or PV gradient (or potentially even the EL). Plumb et al. (2000) indicated that tracer relationships identify complex mixing occurring across the NH vortex barrier, which if ignored may, for example, lead to overestimates of ozone depletion. Developing improved representation of vortex internal structure based on bifurcation may assist in addressing such issues.

Seasonal patterns and differences between the selected years are further examined by assessing standardized maximum metric values every 10 days (Figure 3.10-first column) for the three selected years. The metrics are standardized between 0-1, while EL and CC are inverted (following the approach of Lawrence and Manney 2017). This comparison again shows the coarseness of both EL and CC in determining the strength of the vortex - where they each display a much more 'binary' presence of the vortex barrier - which is shown as being present throughout almost the entire period with little variability in barrier strength. The date of occurrence of the maximum is thus only usefully able to be extracted from $M$ and $\kappa$. Moreover, for EL and CC, the vortex barrier builds quickly from about 1 April, and is usually close to its maximum values within 4-6 weeks. In contrast, $\kappa$ and $M$ build much more slowly from about 1 April, typically reaching their seasonal maximum after 3-6 months.

There are similarities in the shape of the seasonal curves for maximum $M$ and $\kappa$ in Figure 3.10 (left column), particularly late in the season, with a sharp decline from mid to late October. However, $\kappa$ shows more variability throughout each season, particularly in 2014, where it builds quite steadily to a peak in late October when $M$ is already reducing. In contrast $\kappa$ peaks earlier than $M$ in 1980 and both peak at around the same time in 1999. Such differences are likely to be highly influenced by the integration time period of 

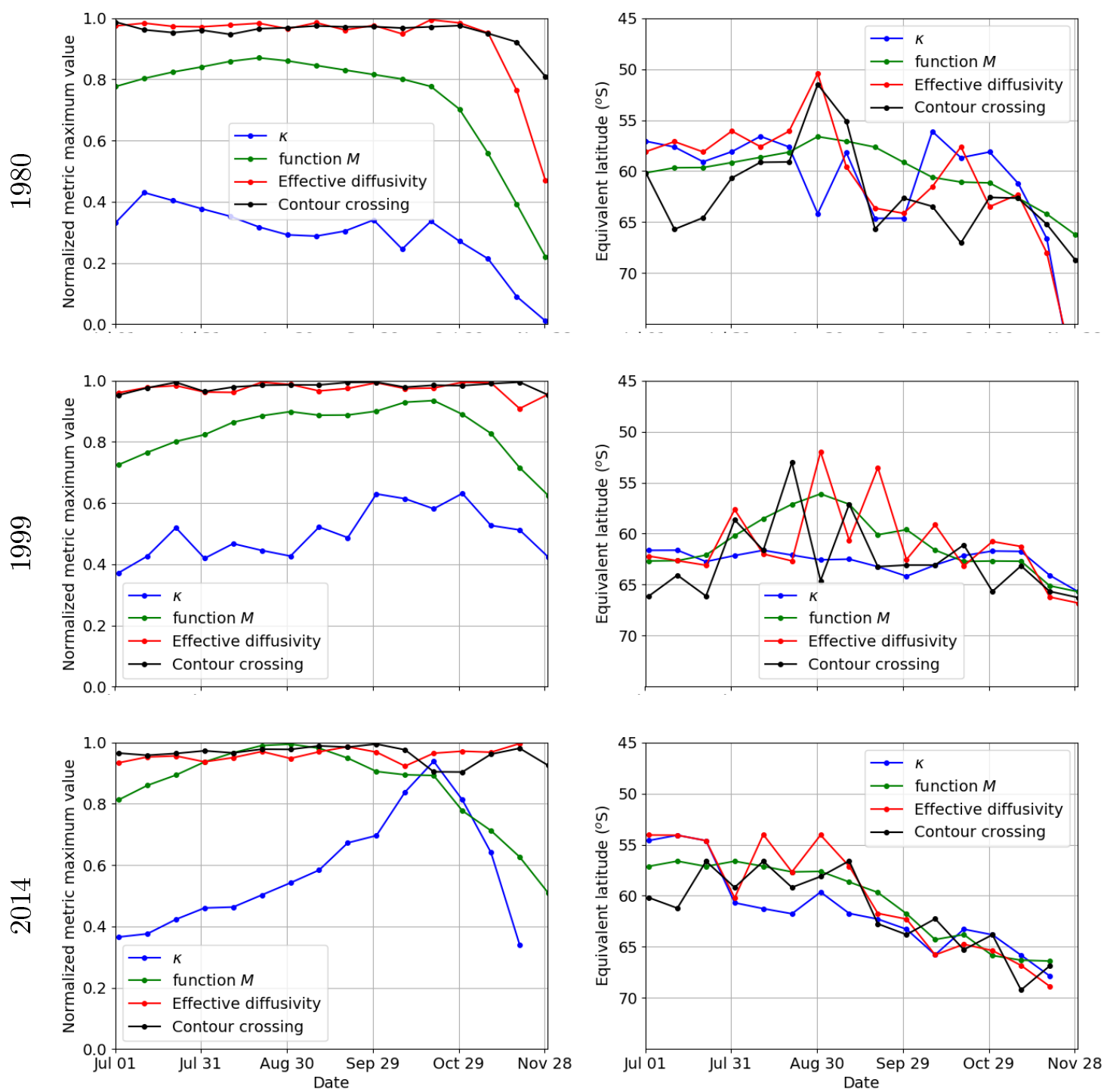

Figure 3.10: Comparison of standardized maximum values (left column) and the equivalent latitude of the maximum value (right column) every 10 days for the years shown, for $\kappa$ (blue), EL (red), $M$ (green), and CC (black). Data are from NCEP-CFSR at $550 \mathrm{~K}$. 
20 days for $M$ as compared with the single day values of $\kappa$. Further analysis would be needed to confirm this, including the examination of several decades of data, to assess what the likely drivers of the peak timing for different metrics are.

The $\phi_{e}$ of the maximum metric values (Figure 3.10-second column) shows consistency between all four metrics across the vortex season for each year. The tendency is for the 'strong core' of the vortex to move poleward over the course of each season (as the size of the vortex reduces), at least for the three selected years shown here. EL and CC have the greatest variability in this measure, associated with their tendency to have many values at or close to zero.

Measurement of the width of vortex barrier (using FWHM) proved difficult for both CC and EL due to frequent occurrence of one-sided profiles (where the half-height value does not appear on each side of the distribution, meaning that a curve width cannot be calculated). Where data are available for comparison, all metrics show a wider barrier earlier in the season and narrowing toward the end of the season (not shown). However, only $M$ and $\kappa$ demonstrate a concomitant reduction in vortex strength throughout the season, suggesting that those metrics offer additional insight into vortex evolution compared to either CC or EL.

$\kappa$ has the narrowest vortex barrier based on FWHM, with typical values of around $10^{\circ}$ near the start of the season, decreasing to about $5^{\circ}$ near the end of the season. FWHMs are similar for the other three metrics, typically around $25^{\circ}$ near the start of the season, decreasing to around $12^{\circ}$. However, care must be taken here, because FWHM is only one possible measure of the barrier width, and has limitations related to one-sided distributions as already seen. Indeed, visual inspection of Figures 3.8 and 3.9 suggests that all four of the metrics robustly represent the barrier width. Comparisons of vortex barrier width based on established vortex edge definitions may show results that differ from those found here using FWHM.

\subsubsection{Metric correlations}

Correlations between pairs of metrics were examined, including additional comparisons with PV gradient and wind-speed. Scatter plots of these correlations for each of the three selected years are provided in Figures 3.11, 3.12, 3.13 and 3.14 .

Data are daily averages at $550 \mathrm{~K}$ for the period 1 July to 30 November (from NCEP-CFSR reanalysis) for all equivalent latitudes between $50-70^{\circ} \mathrm{S}$. Note that some of the plots are of lower 'density', given that they are based on trajectories that are run only every 10 days (i.e., for $M$ and CC). Moreover, 
comparisons are made between metrics that are 'instantaneous' (i.e., daily average values), and those that are integrated over periods of 10-20 days (i.e., trajectory-based), which may also generate anomalies in the relationships explored here.

Curve fitting to the metric comparisons is undertaken with a package produced for this purpose within the Python programming language (Scipy curvefit), which uses a least-squares minimization approach to optimize the fit of a function to a data set. First, an appropriate curve type is selected based on the appearance of the relationship between the two metrics. The correlations between metrics indicate several different curve types or functional relationships, including apparent exponential, harmonic, hyperbolic, generalised logistic function, and polynomial. These were tested against the data and the best fit for the selected curve type included on the scatter-plots, together with the Spearman correlation coefficient. The functions applied for each plot series (i.e., each row) are shown in the Figure captions. In addition, the same metric comparisons were undertaken to show variation by equivalent latitude (instead of by time period). These are included at Appendix A and are discussed along with the patterns observed for all correlations below.

\section{Equivalent Length (EL)}

Figure 3.11 provides the EL comparisons with $\kappa$ (top row), CC (2nd row), PV gradient (3rd row), and wind-speed (4th row). For $\kappa$, PV gradient and windspeed, a negative exponential-form relationship is apparent. High values of EL are related to increasingly near zero values of $\kappa$ and PV gradient, and tend toward wind-speeds of $\sim 10-15 \mathrm{~ms}^{-1}$. For EL vs $\kappa$ and EL vs wind-speed these patterns appear highly correlated with the time of season, where for a given level of EL, both $\kappa$ and wind-speed are lower late in the season than earlier in the season.

This hysteresis pattern is indicative of a 'memory' in one or both of the comparison metrics over the season. In the case of EL, the vortex forms out of the SH's relatively quiescent summer extratropical stratosphere with little or no planetary wave activity. Thus EL is low to moderate as the vortex begins to form and $\kappa, M$, and wind-speed increase. EL is at its minimum mid-season when those other metrics are high. Toward the end of the vortex season, EL increases dramatically to reach its maximum (i.e., peak waviness of the flow), as the vortex breaks-up. Hysteresis may be defined as irreversible thermodynamic change - so the evolution of the vortex as it forms is found to be quite different from the breakup of the vortex at the end of its season.

Interestingly, a change in the maximum value of EL between the comparison years is seen late in the season (i.e., maximum of about 4.3 in 1980 
$1 \cap 0 \cap$
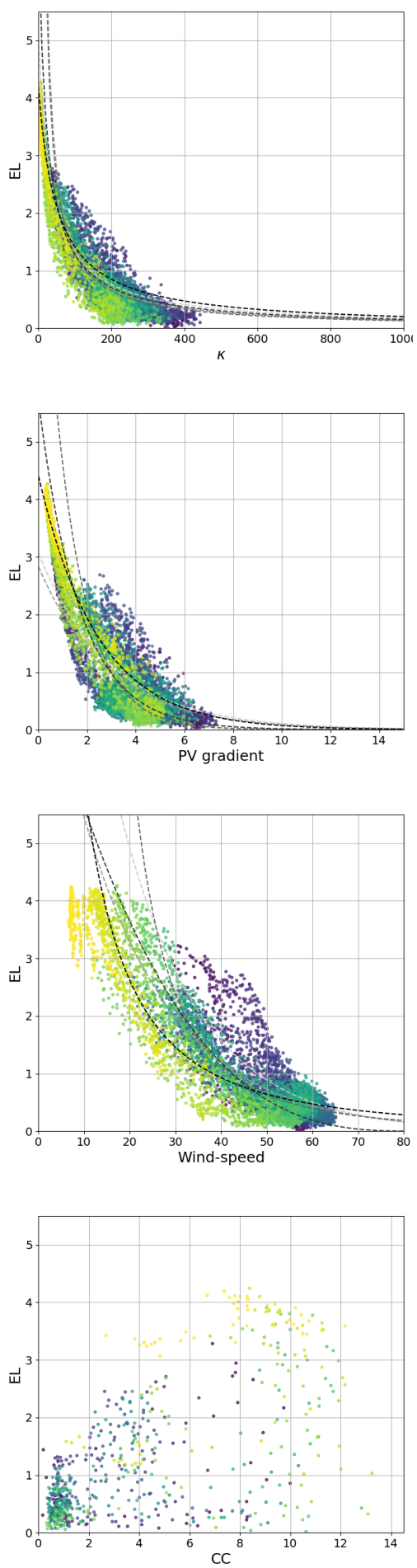

$1 \mathrm{n} n \mathrm{n}$
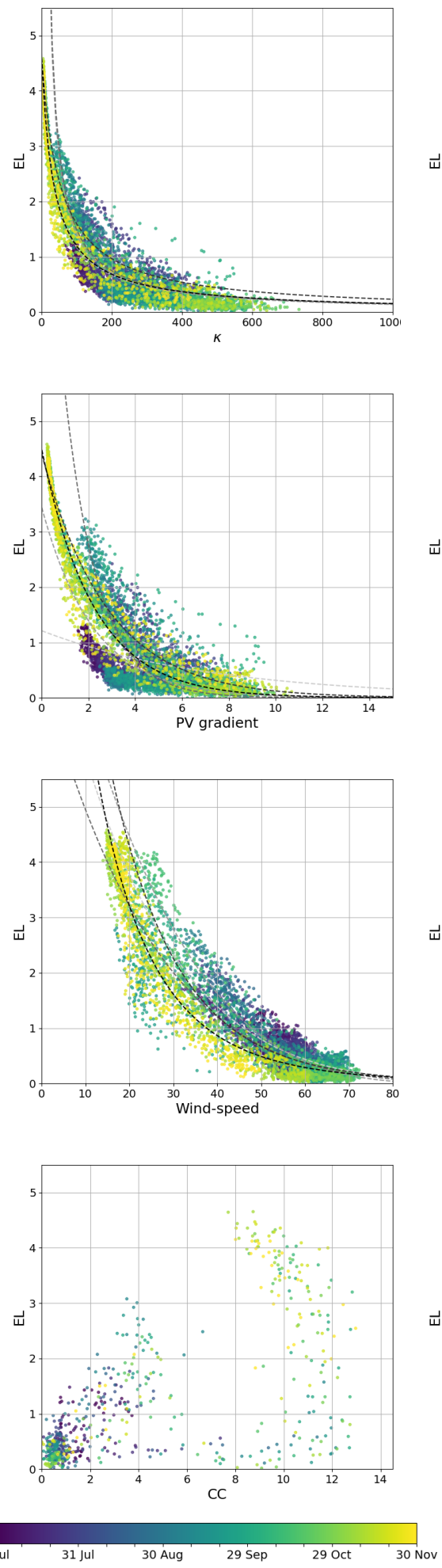

$\circ \cap 11$
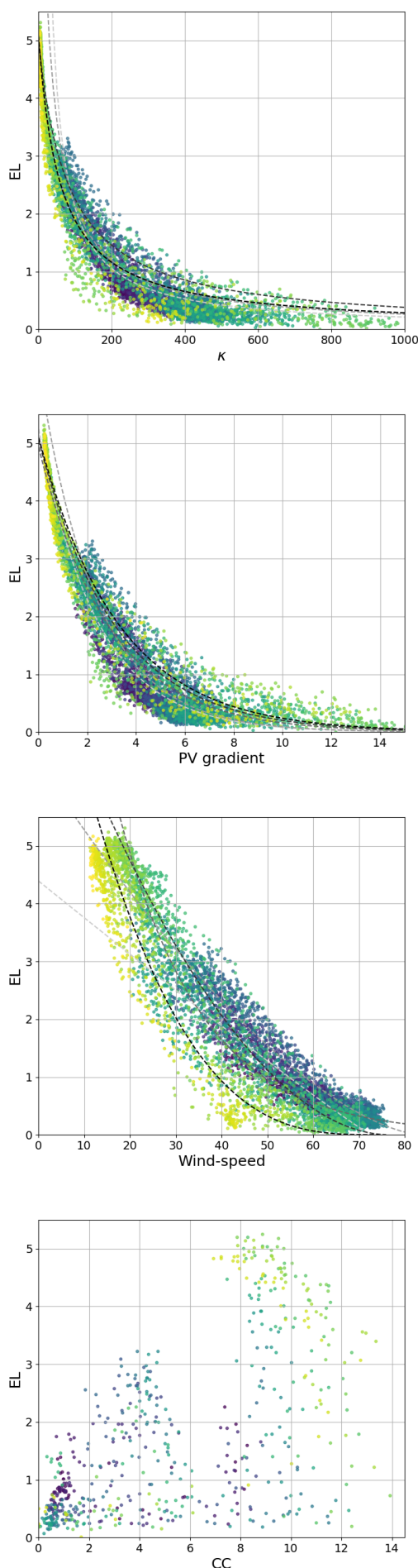

Figure 3.11: Correlation of mixing diagnostics at the $550 \mathrm{~K}$ level for daily data from NCEP-CFSR for the period from 1 July-30 November (colour scale) for 1980 (left), 1999 (middle), and 2014 (right). Comparisons are for EL vs $\kappa$ (top - harmonic), EL vs PV gradient (2nd row - exponential), EL vs wind-speed (3rd row - hyperbolic), and EL vs CC (bottom - no functional fit established) for the region between $50-70^{\circ} \mathrm{S}$ (at $0.5^{\circ}$ increments). The coloured dashed lines show the curve fits for the given functions for 30-day periods (lightest gray for Jul, darkest gray for Nov). 
and about 5.3 in 2014). The increased EL corresponds to periods of low PV gradient and $\kappa$ (i.e., the absence of a vortex), and also to an increasing steepness of the curve, where, for example, a wind-speed of $20 \mathrm{~ms}^{-1}$ in November 1980 corresponded with an EL of about 2.5, but the corresponding value for November 1999 was about 3, and for November 2014 is about 3.5. In 2014, at low wind-speeds, a greater degree of mixing is occurring compared with the other two selected years (i.e., there is more 'waviness' in the flow in the surf zone). Increased mixing in the surf zone could be associated with a stronger vortex barrier in 2014 compared to the earlier years (i.e., greater refraction of waves away from the vortex edge), but further analysis over additional years of data would be needed to confirm this. For CC we see lack of a coherent relationship with EL, as for comparisons of $\mathrm{CC}$ with other metrics (see the following section).

\section{Contour Crossing (CC)}

Figure 3.12 provides comparisons of $\mathrm{CC}$ against $\kappa$ (top row), $M$ (2nd row), PV gradient (3rd row), and wind-speed (4th row). While metric relationships with CC are generally fairly unconstrained, for several of these comparisons there appear to be three distinct CC 'zones' demonstrating varying behaviour in the compared metric. These zones correspond roughly to $\mathrm{CC}$ values that are high (above about 6 ), medium $(\sim 2-6$ ), and low (less than $\sim 2$ ).

The three zones appear able to define the seasonality by representing, in chronological order: an early-season transitional period with high scatter and low correlation (medium CC) corresponding to generally increasing $\kappa, M$ and PV gradient; an early to mid-season period (low CC), associated with a strong vortex barrier, as indicated by high values of $M$ and medium to high values of $\kappa$, and; the late-season vortex break-up (high CC), aligned with low values of $M, \kappa, \mathrm{PV}$ gradient and wind-speed. The late season peak in $\mathrm{CC}$ with wind-speeds around $25 \mathrm{~ms}^{-1}$ indicates that $\mathrm{CC}$ may be effective at identifying the timing of this vortex dissipation period. An apparent gap in $\mathrm{CC}$ exists at values around 6 , aligned with the transition from the stronger vortex to dissipation of the vortex, and suggesting a fairly clear demarcation in $\mathrm{CC}$ between these two phases of the season.

\section{Function $M(M)$}

Figure 3.13 provides comparison of $M$ against $\kappa$ (top row), EL (2nd row), PV gradient (3rd row) and wind-speed (4th row). An additional comparison was undertaken for $M$ against PV and is shown in Figure 3.14 (3rd row) this was included to provide a direct comparison with a similar plot produced 

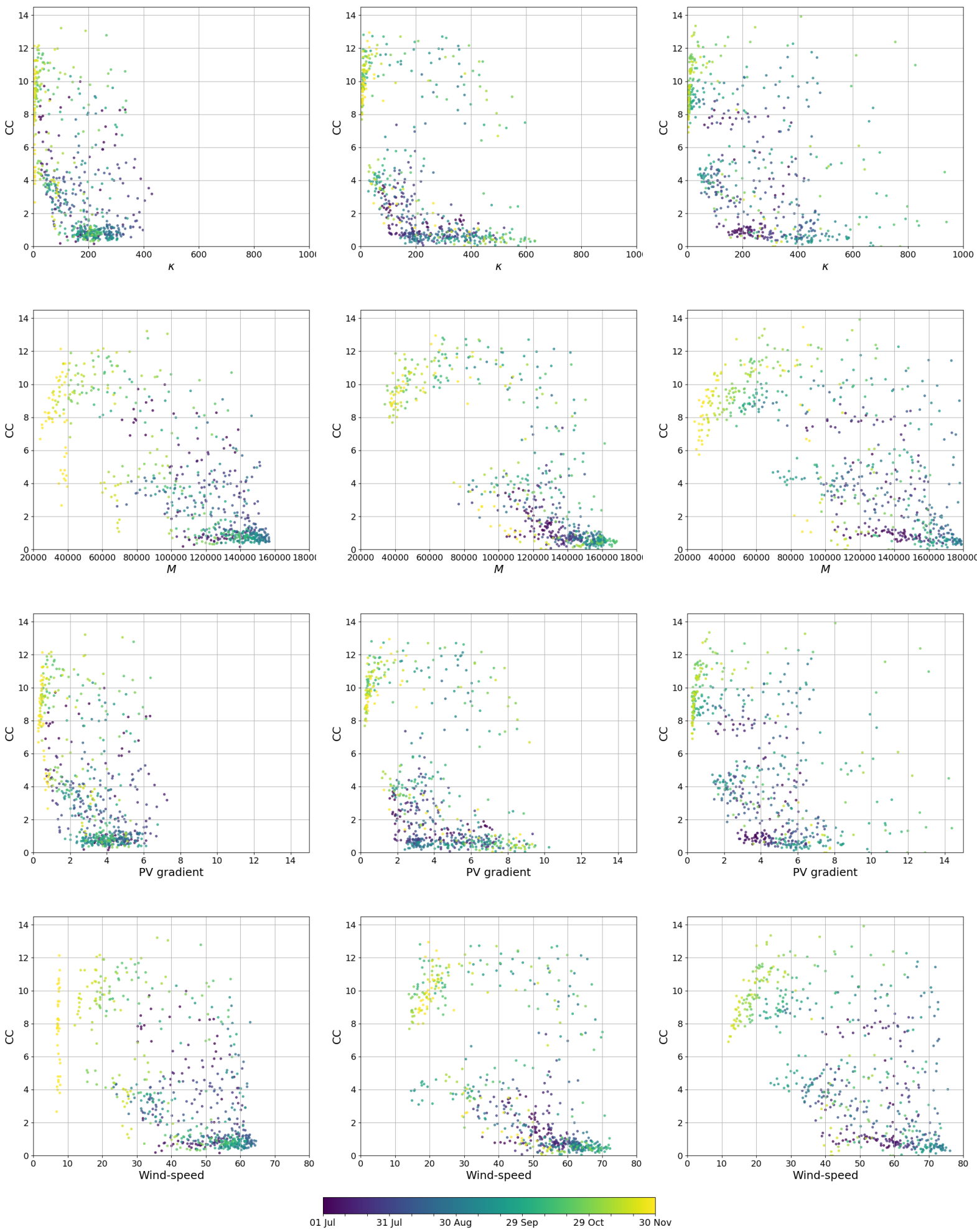

Figure 3.12: As for Figure 3.11 but for CC vs $\kappa$ (top), CC vs $M$ (2nd row), $\mathrm{CC}$ vs PVgradient (3rd row) and CC vs wind-speed (bottom). Note that no functional fits are established between $\mathrm{CC}$ and other metrics compared here. 
for the NH by Manney and Lawrence (2016).

The plots of $M$ against $\kappa$ and PV appear to show a parabolic/hyperbolic relationship. For the $\kappa$ comparison, this manifests as $M$ values reaching a threshold where they flatten out and even begin to decrease, while $\kappa$ values continue to increase. For the PV comparison, the pattern is much clearer, with a series of 'horseshoe' shaped curves, reducing in amplitude and each well aligned with a different time of the season. These curves are somewhat comparable to those shown in Figure 3.9 (given that $\phi_{e}$ is a PV-based coordinate). The horseshoe shape of these curves is consistent with the findings of Manney and Lawrence (2016) in the NH, indicating the PV range associated with the vortex edge (i.e., where the maximum values of $M$ correspond to the edge). They found that the distinctive pattern became less well defined as the vortex weakened, indicating increased mixing. In addition, they observed a 'double-arched' curve, indicating two vortices of different strengths resulting from a stratospheric warming/vortex splitting event. In their work, $M$ was found to reveal variations in the transport barrier around the $\mathrm{NH}$ vortex edge, and to indicate the relative strength and dissolution of offspring vortices that could not be detected in either the PV gradients or effective diffusivity. $\kappa$ and EL also show these distinctive U-shape (or horseshoe) curves when plotted against PV (not shown), again comparable to the plots of these metrics against $\phi_{e}$.

Generally linear relationships are observed between $M$ and EL, and between $M$ and wind-speed, and $M$ and $\mathrm{PV}$ gradient. These result from $M$ being greatest near the vortex barrier (high wind-speeds), where transport is close to circular (low EL), and the PV gradient is steep. This EL comparison also indicates a hysteresis signal, which tracks the formation, development and breakdown of the vortex through the season (as noted above). The early season is associated with higher $M$ values for a given EL, while late in the season a lower $M$ value is found for the same EL. As the vortex breaks-up, $M$ decreases in line with a reduction in wind-speed, before the EL reduces, indicating the persistence of wave action in the surf zone.

\section{Meridional Impermeability $(\kappa)$}

Each of the previous metric comparisons has included a comparison with $\kappa$. Here, additional plots are included in Figure 3.14 that show $\kappa$ compared with PV gradient (top row) and wind-speed (2nd row). For the PV gradient comparison, almost linear relationships are apparent, and these are highly seasonally dependent. The wind-speed comparison indicates more of an exponential relationship, where low values of $\kappa$ are associated with low wind-speed at both the beginning and end of the vortex season, but increas- 

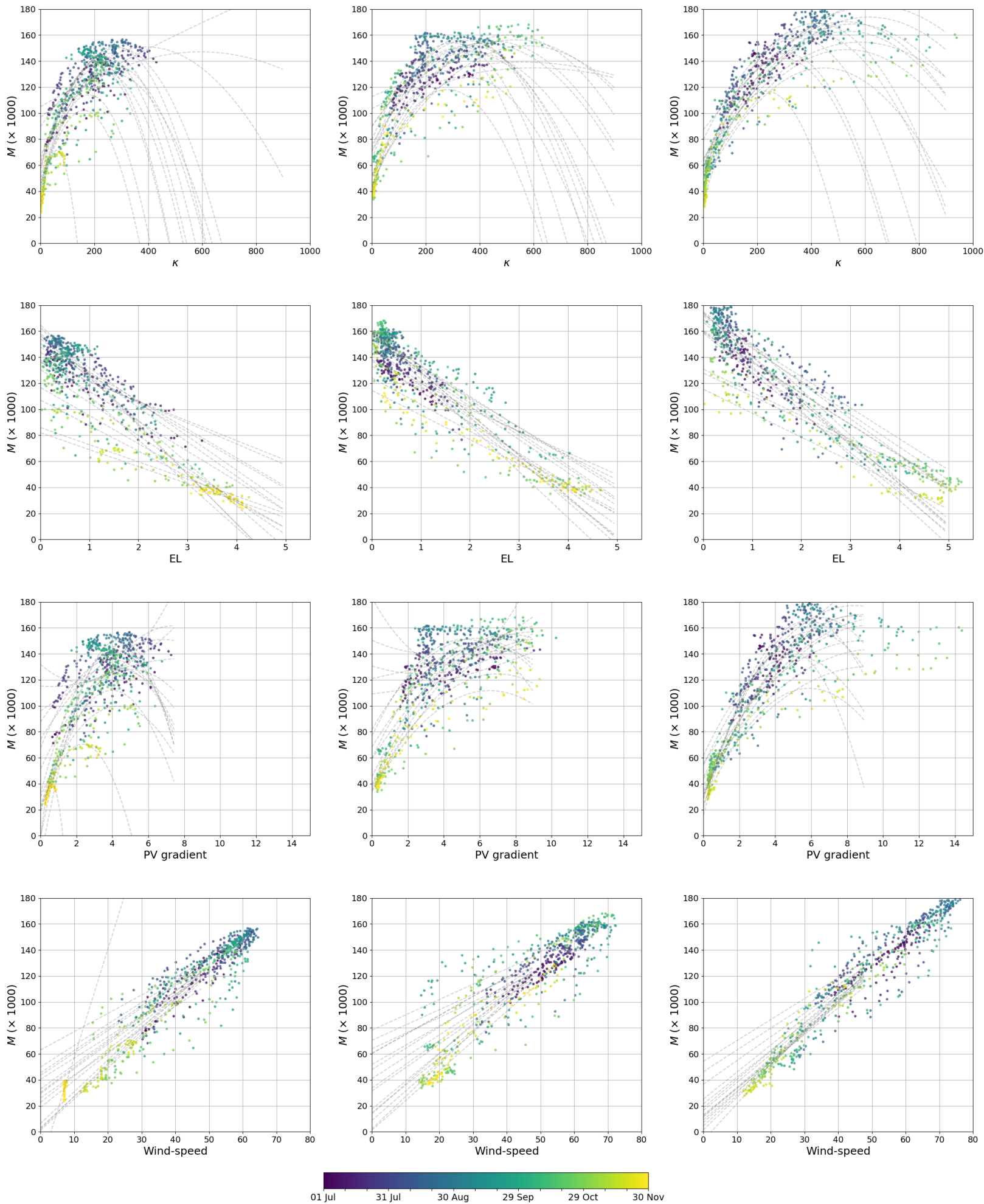

Figure 3.13: As for Figure 3.12 but for but for $M$ vs $\kappa$ (top - polynomial-2 terms), $M$ vs EL (2nd row - linear), $M$ vs PV gradient (3rd row - linear) and $M$ vs wind-speed (bottom - linear). 


\begin{tabular}{|c|c|c|c|c|}
\hline$M$ & 0.76 & \multicolumn{2}{|c}{} \\
\cline { 1 - 2 } CC & -0.23 & -0.26 & \multicolumn{2}{|c}{} \\
\cline { 1 - 2 } EL & -0.98 & -0.82 & -0.45 & \multicolumn{1}{|c}{} \\
\hline PV grad & 0.98 & 0.71 & -0.36 & -0.95 \\
\hline W.spd. & 0.94 & 0.84 & -0.26 & -0.96 \\
\hline & $\kappa$ & $M$ & CC & EL \\
\hline
\end{tabular}

Table 3.2: Summary of Spearman correlation coefficients for mixing diagnostics, averaged over the 3 selected years.

ing wind-speeds near the start of the season lead the increase in $\kappa$ (i.e., the wind-speeds appear to need to build to a threshold level of around $60 \mathrm{~ms}^{-1}$ before the meridional impermeability of the vortex is able to increase beyond about $200 \kappa \mathrm{U}$ ). Late in the vortex season, $\kappa$ requires a higher PV gradient to be maintained as wind-speed reduces (ie., recalling that $\kappa$ is a function of PV gradient and wind-speed) and the barrier width narrows.

It is also apparent that the timing of the maximum wind-speed does not always coincide with the maximum in $\kappa$. At the height of the vortex season, when $\kappa$ reaches its maximum, there are differing relationships with windspeed for each of the three selected years. In 1980, the maximum in $\kappa$ occurs very early in the season (at wind-speeds of about $60 \mathrm{~ms}^{-1}$ ). For 1999, maximum $\kappa$ occurs mid to late season (with winds reaching around $70 \mathrm{~ms}^{-1}$ ). In 2014 maximum $\kappa$ occurs mid-season (where wind-speeds are about $65 \mathrm{~ms}^{-1}$ ), although higher wind-speeds of up to about $75 \mathrm{~ms}^{-1}$ are apparent for lower levels of $\kappa$ (around $400 \kappa \mathrm{U}$ ). These results suggest that high daily and interannual variability are both influential on the seasonal pattern of $\kappa$, and further analysis based on additional years of data would be needed to untangle some of these effects.

The 3-year averages of the Spearman correlation coefficients are summarised for each metric comparison in Table 3.2. For the four metrics, these reveal that the highest correlation is between $\kappa$ and EL, followed by EL and $M$ and then $M$ and $\kappa$. Both $\kappa$ and EL have similar levels of high correlation with wind-speed and PV gradient, while for $M$ the correlation with windspeed is fairly high, but that for PV gradient is lower - as may be expected for a trajectory-based metric that does not assume transport along PV isolines. As we have already seen, CC does not correlate highly with any of the metrics (though the maximum correlation that does exist is with EL). These results suggest that EL and $\kappa$ may be able to act as proxies for the trajectory based metrics, although they appear to identify differing features of the mixing and transport barrier (as discussed below). 

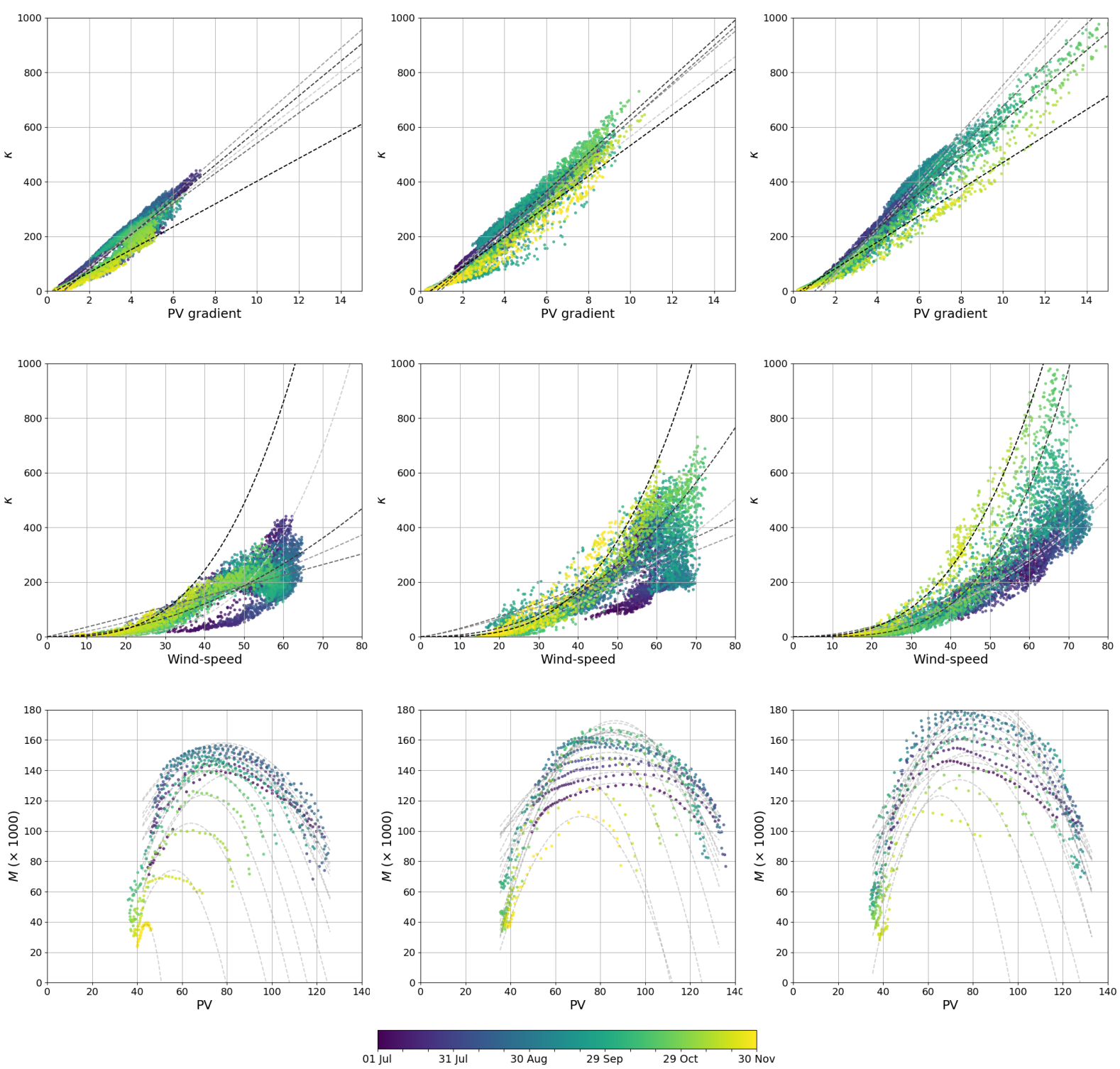

Figure 3.14: As for Figure 3.12 but for but for $\kappa$ vs PV gradient (top linear), $\kappa$ vs wind-speed (2nd row - exponential), and $M$ vs PV (bottom polynomial-2 terms). 
Plots showing variation between metrics by equivalent latitude instead of by the time of the season (see Appendix A) confirm that the absolute maximum value is typically located at around the $64^{\circ} \mathrm{S}$ equivalent latitude (as seen in Figure 3.10 above), which is frequently observed as the approximate vortex edge location during the height of the season. At lower equivalent latitudes of around $50^{\circ} \mathrm{S}$ (i.e., closest to the equator based on the range of $\phi_{e}$ chosen here) we observe maximum values of EL, and minimums in $\kappa$, windspeed, and PV gradient (near the end of the season). On the other hand, the area of higher equivalent latitudes (around $70^{\circ} \mathrm{S}$ ) corresponds to moderate or low values of each metric - which is likely to be due to that region typically being located inside the vortex annulus when it has formed.

\subsubsection{Other levels and reanalyses}

Each of the metrics examined here were also compared using ERA-Interim reanalysis at $550 \mathrm{~K}$ as shown in Appendix A. While most of the comparisons are very similar between the two reanalysis, there are also some differences of note, which are briefly outlined here. For $\kappa$, ERA-Interim appears to show a slightly weaker barrier than NCEP-CFSR, while the EL barrier is considerably wider in ERA-Interim. CC shows a vortex barrier developing earlier in the season in ERA-Interim compared with NCEP-CFSR. For $M$, there are no apparent differences between the two reanalyses.

The same metric comparisons were also undertaken for two additional levels $(700 \mathrm{~K}$ and $850 \mathrm{~K})$, to provide an assessment of differences in vertical structure of the vortex between the metrics. These comparisons are also shown in Appendix A. A larger and stronger barrier is consistently shown by all metrics at higher isentropic levels, typically starting further equatorward than at the $550 \mathrm{~K}$ level, and moving poleward over the course of the season. 


\begin{tabular}{|l|l|l|l|}
\hline Metric & $\begin{array}{l}\text { Formation } \\
\text { (no vortex) }\end{array}$ & $\begin{array}{l}\text { Mid-season } \\
\text { (strong vortex })\end{array}$ & $\begin{array}{l}\text { Break-up } \\
\text { (vortex } \\
\text { dissipation) }\end{array}$ \\
\hline$\kappa$ & Low & Maximum & Moderate \\
\hline$M$ & $\begin{array}{l}\text { Moderate to } \\
\text { high }\end{array}$ & Maximum & Low \\
\hline CC & Moderate & Low & Maximum \\
\hline EL & Moderate & Low & Maximum \\
\hline PV & High & Moderate & Low \\
\hline $\begin{array}{l}\text { Wind- } \\
\text { speed }\end{array}$ & $\begin{array}{l}\text { Moderate to } \\
\text { high }\end{array}$ & Maximum & Low \\
\hline $\begin{array}{l}\text { PV } \\
\text { gradient }\end{array}$ & Moderate & Maximum & Low \\
\hline
\end{tabular}

Table 3.3: Summary of seasonal patterns in mixing diagnostics.

\subsection{Discussion}

A summary of the seasonal evolution of each metric is shown in Table 3.3. Several of the metrics indicate hysteresis patterns when compared against one another, and against wind-speed. This provides insight into the different dynamics occurring during vortex formation (spin-up), 'peak vortex' (i.e., mid-season), and vortex breakup (erosion and dissipation). Distinctive patterns in each metric, over each of the years and across each season, is apparent.

An assessment of Gaussian curve analytics has allowed quantitative comparisons during seasons, between years and between different metrics. These included investigation of maximum (i.e., peak) metric values and their $\phi_{e}$ position, and the width of the vortex barrier (based on the FWHM). Results demonstrated that all four metrics investigated were in good agreement in the meridional location of their maximum values. However, the range in those maximum values across the vortex season was much lower for $\mathrm{CC}$ and EL, making those metrics less effective in determining nuances of the vortex barrier to transport. Furthermore, the dates of occurrence of the maximum values varied widely between the metrics.

Increasing EL values over the three years seen at the end of the vortex season suggest that higher EL, equating to stronger mixing outside the vortex, occurs in tandem with the stronger and longer lasting vortex structure. Further work that examines these relationships would be of interest, particularly looking at how well EL corresponds with vortex strength toward the 
end of the season.

A summary of the overall strengths and weaknesses identified in the four selected metrics is provided in Table 3.4. This includes factors such as their ease of calculation as well as their ability to capture the existence, peak, strength, latitudinal width, seasonality, and mixing characteristics. Overall, each metric is seen to have its benefits, and may be well suited to differing applications. This is discussed further below.

Several areas which were out of scope in this assessment would benefit from further research. Exploring how each metric correlates with factors such as ozone concentration, temperatures across the vortex barrier, and relationships to GPH and planetary waves would be of interest. Such comparisons would provide a way to further test the performance of each metric, including tracking seasonal processes closely associated with the vortex barrier (such as ozone depletion). Some further investigation of relationships between $\kappa$ and ozone is undertaken in Chapter 5 , but the focus in this chapter was on comparisons only between the selected metrics.

Detailed exploration of the edge locations of the mixing barrier - both poleward and equatorward - on the basis of threshold values assigned for each metric would be of interest. Here, we have seen reasonable correlation in the location of the vortex peak across all of the chosen metrics (e.g., in Figure 3.7 and Figure 3.9), although in-depth analysis into the vortex edge location has not been undertaken here. Further work could also involve defining a diagnostic that indicates the presence or absence of a barrier for each metric, and investigating how that diagnostic compares over time and space.

It may be beneficial in future research to evaluate the structure where values are close to zero for both CC and EL, to determine if this can provide finer detail on the vortex barrier and its internal structure. In addition some of the other metrics introduced in this chapter could be examined (such as FTLEs or LCSs) and compared. Investigation into metric patterns and comparisons across a range of additional potential temperature levels may reveal strengths and characteristics of different metrics that were not able to be examined on the basis of assessment at a only three levels and across two reanalyses in this research (see Appendix A for comparisons at the $700 \mathrm{~K}$ and $850 \mathrm{~K}$ level). 


\begin{tabular}{|c|c|c|}
\hline Metric & Strengths & Weaknesses \\
\hline$\kappa$ & $\begin{array}{l}\text { High definition of cross- } \\
\text { vortex structure. Does not } \\
\text { require trajectory model. }\end{array}$ & $\begin{array}{l}\text { Assumes transport only } \\
\text { along PV isolines. Uses } \\
\text { two wind-based compo- } \\
\text { nents, leading to conflation } \\
\text { between PV gradient and } \\
\text { wind-speed. Less effec- } \\
\text { tive than some metrics at } \\
\text { defining mixing. }\end{array}$ \\
\hline$M$ & $\begin{array}{l}\text { Incorporates transport both } \\
\text { along and across PV iso- } \\
\text { lines. Provides detailed } \\
\text { mixing maps. }\end{array}$ & $\begin{array}{l}\text { Aggregation means loss of } \\
\text { detail for short time-periods } \\
\text { and smoothing of the merid- } \\
\text { ional profile. Requires tra- } \\
\text { jectory analysis. }\end{array}$ \\
\hline $\mathrm{CC}$ & $\begin{array}{l}\text { Appears to indicate three } \\
\text { distinct periods during the } \\
\text { vortex season. }\end{array}$ & $\begin{array}{l}\text { Based on only the PV- } \\
\text { crossing component of } \\
\text { transport. High degree } \\
\text { of noise and scatter in } \\
\text { comparison with other } \\
\text { metrics. Appears unable to } \\
\text { determine detailed vortex } \\
\text { characteristics. Requires } \\
\text { trajectory analysis. }\end{array}$ \\
\hline$\overline{E L}$ & $\begin{array}{l}\text { Does not require a trajec- } \\
\text { tory model. Appears use- } \\
\text { ful in determining the surf } \\
\text { zone. }\end{array}$ & $\begin{array}{l}\text { Assumes transport only } \\
\text { along PV isolines. Provides } \\
\text { little information associated } \\
\text { with minimum values (i.e., } \\
\text { when the vortex is strong). }\end{array}$ \\
\hline $\begin{array}{l}\text { Wind- } \\
\text { speed }\end{array}$ & $\begin{array}{l}\text { Key indicator for existence } \\
\text { of a vortex. }\end{array}$ & $\begin{array}{l}\text { Does not provide detail on } \\
\text { the internal vortex struc- } \\
\text { ture. }\end{array}$ \\
\hline $\begin{array}{l}\text { PV gradi- } \\
\text { ent }\end{array}$ & $\begin{array}{l}\text { Key indicator for existence } \\
\text { of a vortex. }\end{array}$ & $\begin{array}{l}\text { May identify multiple vor- } \\
\text { tices over short time peri- } \\
\text { ods. }\end{array}$ \\
\hline
\end{tabular}

Table 3.4: Mixing diagnostics: strengths and weaknesses. 


\subsection{Conclusions}

This research has provided an overview of a range of mixing metrics, followed by an analytical comparison of four selected metrics, with the aim of determining the relative merits of each. Overall, while each of the selected metrics appear able to capture the presence of the ACV barrier, some perform better at defining the nature of the barrier than others. For example, CC does not appear to indicate internal properties of the barrier, as seen for EL and $\kappa$. $\mathrm{CC}$ is thus more limited in defining the vortex mixing barrier, other than determining its presence or absence, although CC may be useful for providing a measure of the speed of vortex formation/dissipation, and appears able to identify three distinct periods across the vortex season.

The analyses undertaken here confirm that $\kappa$ is well correlated with a range of other diagnostic measures of stratospheric mixing. In fact $\kappa$ has some advantages, by providing high definition of the internal and edge structure of the ACV, and by being more straightforward to calculate. Though it is apparent that $\kappa$ does identify features related to mixing, as well as identifying the vortex barrier, it may be considered less suited for determining the mixing regime than metrics that involve trajectories - particularly $M$ which is able to provide detailed maps of mixing that the other metrics examined here are unable to rival (e.g., see maps at Figure 3.4 above). However, at least for the analysis undertaken here, $M$ is unable to provide the same level of detail across the meridional profile of the vortex annulus as $\kappa$ does. $M$ also has the possible drawback of being aggregated over a long-time period, which causes a loss of information for rapid mixing events in the order of 1 day to 1 week. $\kappa$ provides an easily calculated snap-shot of the vortex barrier and its internal structure that does not require sophisticated trajectory calculations.

There is a fundamental difference between metrics calculated using trajectories and those without. Non-trajectory metrics $-\kappa$ and EL - are based on an assumption of transport occurring along isolines of PV, where PV is conserved (which may be considered to be the dominant transport type). Shuckburgh et al. (2009) point out that diagnostic metrics that are based on averaging around a contour of equivalent latitude do not provide information about longitudinal variations in transport and mixing. In contrast, $\mathrm{CC}$ examines instances only where isolines of PV are being crossed by air parcels that is non-conservative effects involving mixing or diabatic processes. It captures mixing processes much more than processes associated with the barrier itself - i.e., it is not able to determine detail of internal vortex structure as the vortex is simply a region where little CC occurs. Dahlberg and Bowman (1995) find that CC characterises air parcel transport and mixing processes 
associated with ejection of vortex air and entrainment of mid-latitude air into the vortex, rather than determining the vortex barrier itself. With regard to $M$, it does not rely on $\mathrm{PV}$ at all, being only a function of air parcel velocity integrated over time, thus including transport that occurs both along and across PV isolines. Indeed, previous work using $M$ has often focussed on identification of detailed mixing characteristics, including LCSs manifolds and saddle points (Smith and McDonald, 2014) - suggesting that the analysis of such mixing processes is a strength of $M$.

While some of the findings here have been reported previously, many of them are new. As discussed earlier, Manney and Lawrence (2016) observed profiles of mixing metrics by equivalent latitude for the $\mathrm{NH}$ vortex barrier, and developed a comparison between $M$ and PV. Many more additional metric comparisons are undertaken here, revealing new insights into the nature of the barrier and vortex form. In subsequent chapters, the vortex is further investigated with a focus on the period between July and September, when the vortex is at its maximum strength. Particular attention is paid to the month of September, when maximum ozone depletion typically occurs, and the vortex is still strong enough to be important part of that ozone depletion process. 


\section{Chapter 4}

\section{The vortex barrier in the Unified Model}

Chapter 2 outlined the important role of SH stratospheric processes in driving regional and global climate, and in determining how the region will respond to future climate change and recovery from the effects of ODSs. This chapter examines the ability of a selected atmospheric GCM - namely the UK Met Office Unified Model (UM) - to simulate the stratospheric dynamical processes that determine the morphology and hence impermeability of the $A C V$. Two different configurations of the UM from different model generations are used, and are compared with both NCEP-CFSR and ERA-Interim reanalyses. The meridional impermeability metric is used here to determine the extent to which the vortex acts as a barrier to meridional transport.

The UM is widely used internationally, including being the model at the core of the New Zealand Earth System Model (NZESM) that underlies projections of future climate for New Zealand. Findings that identify possible shortcomings in the UM may also be relevant to other global climate models, which could thus also benefit from addressing such behaviour in their configurations.

A paper outlining the results of this comparison, titled "Simulating the Antarctic stratospheric vortex transport barrier: comparing the Unified Model to reanalysis" was published in Climate Dynamics in January 2019 (Cameron et al., 2019), and this chapter is based broadly on that publication. 


\begin{tabular}{|l|l|l|l|}
\hline Configuration & $\begin{array}{l}\text { Dynamical } \\
\text { core }\end{array}$ & $\begin{array}{l}\text { UM code } \\
\text { base }\end{array}$ & References \\
\hline GA3.0 & New Dynamics & 7.8 & $\begin{array}{l}\text { Davies et al. 2005 } \\
\text { Walters } \text { et al. 2011 }\end{array}$ \\
\hline GA7.0 & END-Game & 10.3 & $\begin{array}{l}\text { Wood } \text { et al. 2014 Wal- } \\
\text { ters } \text { et al. 2018 }\end{array}$ \\
\hline
\end{tabular}

Table 4.1: Overview of the two Unified Model configurations used in experiments described in this Chapter.

\subsection{Methods}

\subsubsection{The Unified Model}

Developed by the UK Met Office, the Unified Model (UM) is used in both weather forecasting and climate modelling. The weather forecasting component was developed in the 1960s, climate modelling was added in the 1970s, and these two elements unified in the 1990s (Brown et al., 2012).

The dynamical core for both configurations of the UM used here is based on a scheme with semi-implicit time integration and semi-Lagrangian advection to solve the equations of motion and model atmospheric transport (Davies et al. 2005; Brown et al. 2012; Wood et al. 2014). The equations are discretised horizontally on an Arakawa-C latitude-longitude grid, and vertically on a Charney-Phillips staggered grid using a terrain-following vertical coordinate. An overview of the development of the UM over a 25-year period, with a particular focus on the unification of numerical weather prediction (NWP) and climate modelling is provided by Brown et al. (2012).

In New Zealand, the UM forms the core of the NZ Earth System Model (NZESM) which is currently under development at NIWA, with a horizontal atmospheric resolution of $100 \mathrm{~km}$. Output from the NZESM can be downscaled using a regional climate model, with a typical resolution of $12 \mathrm{~km}$.

The UM is used here to examine vortex impermeability in comparison with reanalyses using two UM versions with differing code bases, dynamical cores, and global atmosphere-land configurations, as shown in Table 4.1. Simulations were carried out by Stephen Stuart at NIWA, Wellington. The UM code base provides the version of the computer code used in the model configuration and thus defines the overall model version. The Global Atmosphere (GA) version is the model configuration representing atmospheric processes, with improvements in the GA over time indicated by higher ver- 
sion numbers. The GA component of the model incorporates both model dynamics (i.e., processes simulated at grid-scale) and model physics (e.g., parameterizations at a range of scales). Physical processes that cannot be explicitly resolved, and are therefore parameterized in the UM, include: cloud cover fraction and condensate within each grid box, as well as precipitation, convection and sub-grid scale gravity wave drag, all of which have been updated in GA7.0 relative to GA3.0.

Within the GA, the dynamical core drives the atmospheric dynamics. It has been found that compared with the New Dynamics core, the ENDGame dynamical core has improved scalability - that is, it is able to be run over an increased number of processors. In addition ENDGame has increased numerical stability - that is, there is improved integration between the atmosphere and the surface based on the use of multiple iterations for each time step (Walters et al., 2017). These factors have negated the need for grid-scale horizontal diffusion and polar filtering in the ENDGame core. The first of these processes accounts for small-scale energy transfers, while the second accounts for wave interaction near the singularity at the poles. These changes further improve stability and scalability and in combination lead to more intense weather systems and significantly improved model performance in the ENDGame dynamical core (Walters et al., 2017).

Misrepresentation of the ACV that has been observed in several climate models, is thought to be largely caused by the parameterization of both nonorographic (e.g., Hardiman et al. 2017) and orographic gravity waves (e.g., Garcia et al. 2017). Orographic gravity waves (also known as mountain waves), can have a considerable influence on the large-scale circulation, and are typically parameterized in climate models. Mountain ranges in the $\mathrm{SH}$, such as the southern Andes and the Antarctic Peninsula are thought to contribute to a significant peak in gravity wave activity in that region (Vosper, 2015). The so called 'cold pole' problem, where the current generation of climate models have a tendency to show a cold bias in the polar stratosphere in the southern winter and spring, is also thought to result from inaccuracies in wave drag parameterizations used in models (McLandress et al., 2012). In addition, the 'small island problem' occurs when wave drag due to the presence of small islands in the flow is not included in climate models. This wave drag is generated at low levels in the atmosphere from upwind flow blocking (i.e., due to direct blocking by low level orography) resulting in flow splitting, wake formation and low-level wave breaking (Vosper, 2015).

A parametrization scheme that improves the representation of flow blocking, based on 'bluff body dynamics', accounting for the enhanced drag that occurs when the low-level flow is approximately normal to the major axis of 
the sub-grid orography was developed by Vosper (2015). The new scheme allows for flow blocking to be treated more independently of grid-scale mountainwave drag (which is based on propagation of linear gravity waves).

In the UM, GA7.0 uses the improved Vosper (2015) 5A scheme, while GA3.0 uses the earlier 4A scheme(Webster et al., 2003). While the 4A scheme includes flow-blocking in its parameterization of sub-grid scale orography, it does not provide for the enhanced flow blocking parameterization developed in the 5A scheme. Sub-grid scale gravity wave drag from non-orographic sources, such as convection, fronts and jets, is represented in both UM configurations by a spectral parameterization (Scaife et al., 2002), which has undergone tuning through successive versions of the model.

Gaseous absorption of radiation is also parameterized via the correlated- $k$ method, with GA7.0 using newly derived absorption coefficients for all gases (Walters et al., 2018). The time between full radiation calculations is reduced from 3 hours in GA3.0 to 1 hour in GA7.0, which improves the treatment of the diurnal cycle in the GA7.0 configuration (Walters et al., 2017). For both configurations, the resolution is N96 in the horizontal plane $\left(1.25^{\circ}\right.$ latitude by $1.875^{\circ}$ longitude), with 85 vertical levels and the model top at $85 \mathrm{~km}$.

\subsubsection{Model simulations}

UM simulations were performed for the 1983-2015 period on a 360-day calendar using 20-minute time steps, with observed sea surface temperatures (SSTs) and sea ice as the lower boundary conditions. Daily SST and sea ice concentrations (Reynolds et al., 2007) were sourced from the Optimal Interpolation SST (Version 2) data set provided by the National Oceanic and Atmospheric Administration (NOAA).

The simulations used in this study do not incorporate interactive stratospheric chemistry, but rather prescribe a monthly-mean ozone field, which is time-evolving and three-dimensional (Checa-Garcia et al., 2018). This ozone boundary condition was prepared for use in Phase 6 of the Coupled Model Intercomparison Project (CMIP6).

Model daily-mean fields (calculated from the models 20 minute time steps) of potential vorticity $(\mathrm{PV})$, zonal wind $(\mathrm{u})$, meridional wind $(\mathrm{v})$, pressure (p) and temperature (T) were output on Arakawa staggered grids, which were transformed to a single common horizontal grid (that used by the PV field) using bilinear interpolation. Model and reanalyses fields are then vertically interpolated using simple linear interpolation to potential temperature levels appropriate for comparison with reanalysis, namely - $395 \mathrm{~K}, 400 \mathrm{~K}$, $430 \mathrm{~K}, 450 \mathrm{~K}, 475 \mathrm{~K}, 530 \mathrm{~K}, 550 \mathrm{~K}, 650 \mathrm{~K}, 700 \mathrm{~K}, 850 \mathrm{~K}, 1000 \mathrm{~K}, 1250 \mathrm{~K}$, and $1500 \mathrm{~K}$. 
The $550 \mathrm{~K}$ level is the primary focus of the analysis undertaken in this chapter, to allow for comparison with the results of Bodeker et al. (2002), where $550 \mathrm{~K}$ was selected as representing the approximate level of peak ozone concentration in the stratosphere. Results for other levels are standardised to the $550 \mathrm{~K}$ level (see section 2.5.1). Results for September are discussed in detail here, while analysis for additional months and isentropic levels are provided at Appendix B. The output from model simulations is compared with both NCEP-CFSR and ERA-Interim reanalyses. Further details on those reanalyses are provided in section 2.4.

\subsection{Results}

\subsubsection{Climatology}

September monthly-mean climatological profiles (1983-2015) by equivalentlatitude $\left(\phi_{e}\right)$ of wind-speed, PV, PV gradient and $\kappa$ on the $550 \mathrm{~K}$ level are compared between the reanalyses and the UM simulations in Figure 4.1. Note that by convention $\mathrm{PV}$ is negative in the $\mathrm{SH}$, so the absolute maximum of $\mathrm{PV}$ is used for ease of interpretation here. The monthly standard deviation over the 33-year period is shown for each meridional profile by the corresponding shaded colour in the figure ( $1 \sigma$ either side of the mean). While overlap of the shaded significance levels in the plots provide the first indication of where the curves in Figure 5.1 may be significantly different from one another, a standard t-test was also carried out to compare each of the model simulations and reanalyses for the wind-speed and $\kappa$ plots. For both variables, significant difference in the distribution was only found between GA3.0 and the reanalyses/GA7.0. However, as seen below, there are some differences in the shape of these $\phi_{e}$ profiles that are of note.

For the $550 \mathrm{~K}$ potential temperature level and the month of September the zone of high wind-speeds (Figure 4.1a) associated with the ACV has a significantly ${ }^{1}$ wider meridional extent in the poleward direction in both UM versions compared to the reanalyses. GA3.0 displays a significantly lower peak wind-speed.

The climatological zonal-mean PV (Figure 4.1b) shows a narrower zone of steep PV gradients in the reanalyses, associated with the corresponding narrower zone of elevated wind-speeds. The reanalyses both show a slight flattening in the PV gradient at around $60^{\circ} \mathrm{S}$ equivalent latitude, though this is so slight that it is difficult to observe in the figure. This slightly flattened

\footnotetext{
${ }^{1}$ Hereafter when two quantities are referred to as being significantly different, that refers to a significant difference at the $1 \sigma$ level
} 

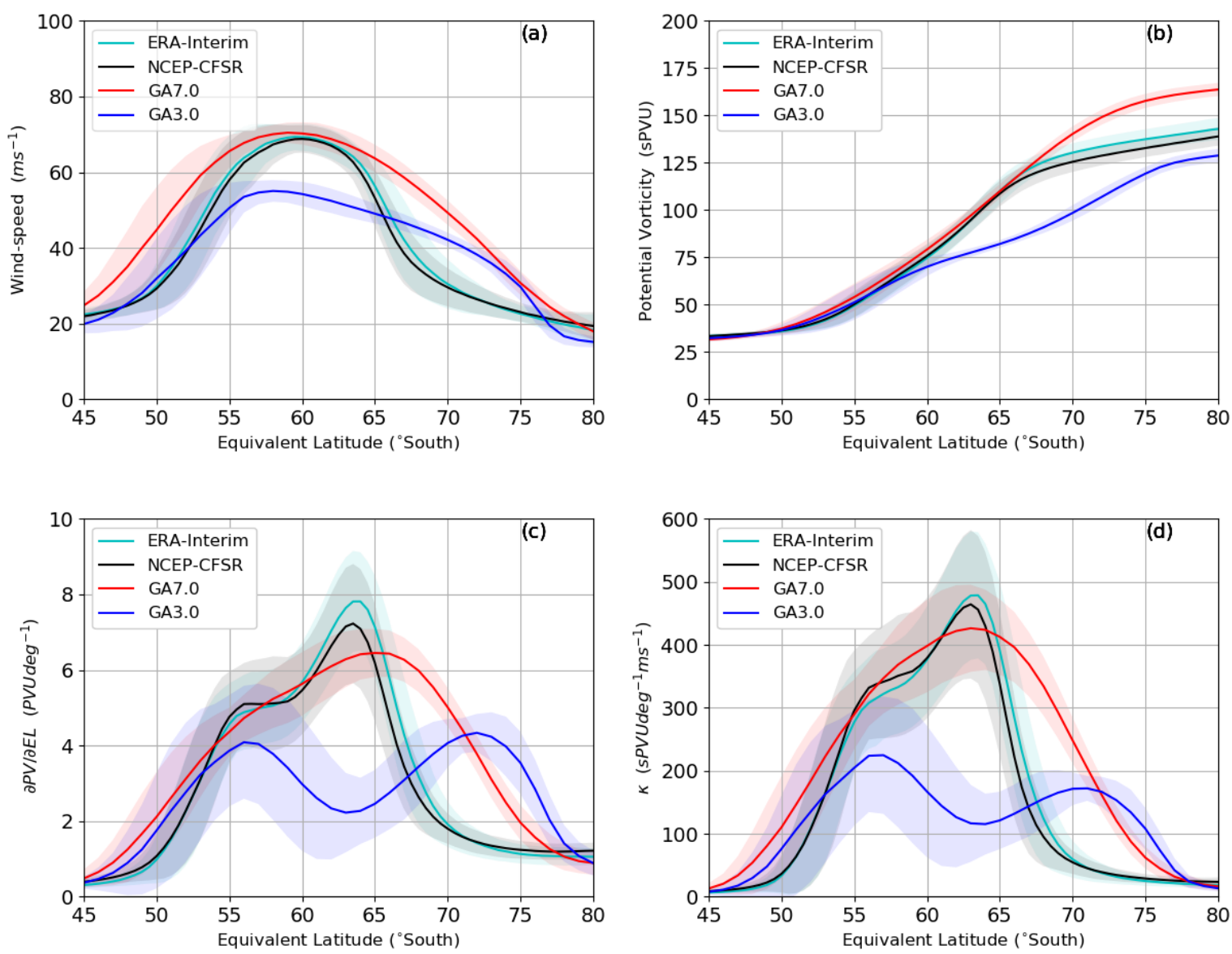

Figure 4.1: September monthly-mean climatologies (1983-2015) of (a) windspeed, (b) PV, (c) $\partial P V / \partial \phi_{e}$, (d) $\kappa$, against equivalent latitude at $550 \mathrm{~K}$ for GA3.0 (blue), GA7.0 (red), NCEP-CFSR (black) and ERA-Interim (cyan). The shaded areas denote the spread of values at $1 \sigma$ either side of the mean. 
PV gradient corresponds to the equivalent latitude of the peak wind-speed in the reanalyses. PV in GA3.0 is significantly different from the reanalyses, and displays two distinct zones of steep meridional gradients separated by a flatter PV gradient at about $64^{\circ} \mathrm{S}$ equivalent latitude. GA7.0 shows a steep $\mathrm{PV}$ gradient which is much more well aligned with reanalyses, but continues further poleward, eventually flattening out from about $73^{\circ} \mathrm{S}$.

The structure of the zonal-mean wind and PV (by equivalent latitude) leads to calculated values of PV gradient (Figure 4.1c) and $\kappa$ (Figure 4.1d). Though there is little difference between the monthly averaged PV gradient and the $\kappa$ curve shapes, investigation of data on a daily basis (not shown) indicates that more of the variability is due to variation in the wind-speed, than the PV gradient. Representation of $\kappa$ and PV gradient in GA7.0 only becomes significantly different from the reanalyses at equivalent latitudes between $\sim 67^{\circ} \mathrm{S}$ and $\sim 78^{\circ} \mathrm{S}$, showing a stronger barrier on the poleward flank of the vortex. Overall, both PV gradient and $\kappa$ profiles are poorly represented in GA3.0.

\begin{tabular}{|l|c|c|c|c|c|}
\hline & Source & $\mathrm{U}$ & $\mathrm{PV}$ & $\partial P V / \partial \phi_{e}$ & $\kappa$ \\
\hline Mean & GA3.0 & 36 & -84 & 2 & 106 \\
& GA7.0 & 45 & -105 & 3 & 191 \\
& CFSR & 37 & -95 & 3 & 142 \\
& ERA-Int & 38 & -97 & 3 & 144 \\
\hline Maximum & GA3.0 & 55 & -132 & 4 & 225 \\
& GA7.0 & 71 & -169 & 7 & 426 \\
& CFSR & 68 & -147 & 7 & 464 \\
& ERA-Int & 69 & -149 & 8 & 479 \\
\hline FWHM & GA3.0 & $27^{\circ}$ & - & $26^{\circ}$ & $24^{\circ}$ \\
& GA7.0 & $26^{\circ}$ & - & $21^{\circ}$ & $18^{\circ}$ \\
& CFSR & $17^{\circ}$ & - & $13^{\circ}$ & $12^{\circ}$ \\
& ERA-Int & $18^{\circ}$ & - & $13^{\circ}$ & $12^{\circ}$ \\
\hline
\end{tabular}

Table 4.2: Metrics for the September climatologies at $550 \mathrm{~K}$ shown in Figure 4.1. Units are $\mathrm{ms}^{-1}$ for mean and maximum wind-speed, $P V U$ for $\mathrm{PV}$, PVU.deg ${ }^{-1}$ for the PV gradient and PVU.deg ${ }^{-1} \cdot m s^{-1}$ (hereafter $\kappa \mathrm{U}$ ) for $\kappa$. Note that FWHM values are in degrees of equivalent latitude and are unable to be calculated for PV curves as they are non-Gaussian. Values are calculated over the region from $45-85^{\circ} \mathrm{S}$ equivalent latitude.

FWHM is used to determine the equivalent latitude range of the distributions of wind-speed, PV gradient and $\kappa$. Issues can arise in determining 
FWHM when the distribution is non-Gaussian. For example, if there are multiple peaks then there may be several points on the curve that match the 'half-maximum' value. Here, if multiple peaks are apparent the full width is taken based on the outermost half-maximum values. Another issue appears if the distribution is highly skewed, so that the half-maximum value may not appear on that side of the distribution within the range of equivalent latitudes chosen $\left(45-85^{\circ} \mathrm{S}\right.$ here), in which case FWHM values are not able to be calculated.

Table 4.2 provides a summary of the mean, maximum and FWHM values for wind-speed, PV, PV gradient and $\kappa$ for the traces shown in Figure 4.1. The UM $\kappa$ profile for GA7.0 (Figure $4.1 \mathrm{~d}$ ) is $\sim 6^{\circ} \phi_{e}$ wider at FWHM than the reanalyses, with this difference primarily due to higher $\kappa$ values continuing poleward in GA7.0. The FWHM for $\kappa$ in GA3.0 is around $12^{\circ} \phi_{e}$ wider than the reanalysis. Similar patterns for FWHM are seen for both wind-speed and PV gradient. Slightly higher means and maximums of wind-speed, PV and $\kappa$ are seen in ERA-Interim compared with NCEP-CFSR, while GA3.0 is consistently lower and GA7.0 consistently higher across these variables.

GA3.0 shows a bifurcated structure across the ACV barrier (Figure 4.1c and $4.1 \mathrm{~d})$, with $\kappa$ peaks at two distinct equivalent latitudes $\left(\sim 57^{\circ} \mathrm{S}\right.$ and $\left.\sim 71^{\circ} \mathrm{S}\right)$. This structure, which results from the two steep zones of PV observed in GA3.0 (Figure 4.1b) is not evident in the climatology for GA7.0, and is thought to be due to aberrant wind fields in the model (see section 4.2.4). However, a secondary hump or 'shoulder' in the $\kappa$ curve is seen in the reanalyses around $60^{\circ} \mathrm{S}$, which corresponds to the slight flattening in the $\mathrm{PV}$ gradient and the maximum of wind-speed mentioned earlier. For the reanalyses, the $\kappa$ 'peaks' occur at around $55^{\circ} \mathrm{S}$ and $63^{\circ} \mathrm{S}$.

An in-depth characterisation of bifurcation tendencies in the meridional impermeability of the ACV structure diagnosed in reanalyses is presented in Conway et al. (2018). They find that a bifurcated or double-walled barrier is common in the ACV, particularly in mid-winter in the mid-stratosphere (between 395-850 K), where strong peaks can occur at the same time, but can also be stronger at one peak location or the other at differing times. Single peaks can also continue to occur without bifurcation. Conway et al. (2018) develop metrics that quantify this bifurcation, and explore the implications for detecting the edge of the vortex barrier. Refer to Figure 3.3 for a bifurcated profile, where the metrics developed by Conway et al. (2018) to characterise the composition and extent of bifurcation are shown. While previous work has examined a vortex edge region of weakly mixed air (e.g., Roscoe et al. 2012), those studies are typically referring to the entire vortex annulus, and comparing it to the stronger mixing that occurs both inside and 
outside the annulus. Here, the bifurcation occurs across the annulus itself, representing a zone where some degree of increased mixing occurs.

Climatological profiles by equivalent latitude are shown for other months on the $550 \mathrm{~K}$ and $850 \mathrm{~K}$ levels in Appendix B. At $550 \mathrm{~K}$ these show a wider barrier in both July and August than seen here in September, with no sign of bifurcation in July, but some suggestion of bifurcation in August. Higher $\kappa$ in GA7.0 can be observed in those months, compared with what was seen here for September. At the $850 \mathrm{~K}$ level a similar pattern is seen, where the GA7.0 $\kappa$ is much higher than the reanalyses, particularly in July and August. These results support the findings discussed here for the $550 \mathrm{~K}$ level in September.

\subsubsection{Monthly versus daily data}

As a result of monthly averaging, it is likely that some key elements of vortex variability are lost. For example, there may be short bursts of planetary wave activity and wave breaking that occur at periods shorter than a month, thus altering the strength and nature of the vortex. In the most extreme case, this type of activity may lead to occurrence of an SSW, although as noted previously (section 2.2.6) these are rare in the SH. In addition, the monthly averaging leads to a loss of definition and the frequency of occurrence of bifurcation. To examine these issues further, the daily variation in $\kappa$ is examined here.

In Figure 4.2 the daily and monthly average equivalent latitude profiles for September of two selected years are shown. The years were chosen on the basis of exhibiting a distinct bifurcation tendency in the reanalyses. Although bifurcated $\phi_{e}$ profiles are also found in GA3.0 and GA7.0, these are thought to be aberrations in the model, as discussed above. The pattern for NCEP-CFSR for these two years shows high variability in the location of the bifurcation which is seen to be a common daily occurrence, while the monthly averaged profile smears out the extent of bifurcation. Other years (not shown) were observed to demonstrate similar patterns. Interestingly, for the reanalyses there is greater variation in the daily $\kappa$ for the selected months for NCEP-CFSR compared to ERA-Interim. This is likely to be due to the finer horizontal resolution in NCEP-CFSR $\left(0.5^{\circ}\right)$ than in ERAInterim $\left(0.75^{\circ}\right)$, which would allow it to display a greater degree of variability across equivalent latitudes. The variation in form of the ACV that occurs over periods of less than a month is an important component of stratospheric dynamics in the SH polar region, with aspects such as the bifurcation tendency (which appears to represent an inherent Lagrangian transport feature associated with jet cores - see Chapter 3) not being well captured in the UM. 
1984
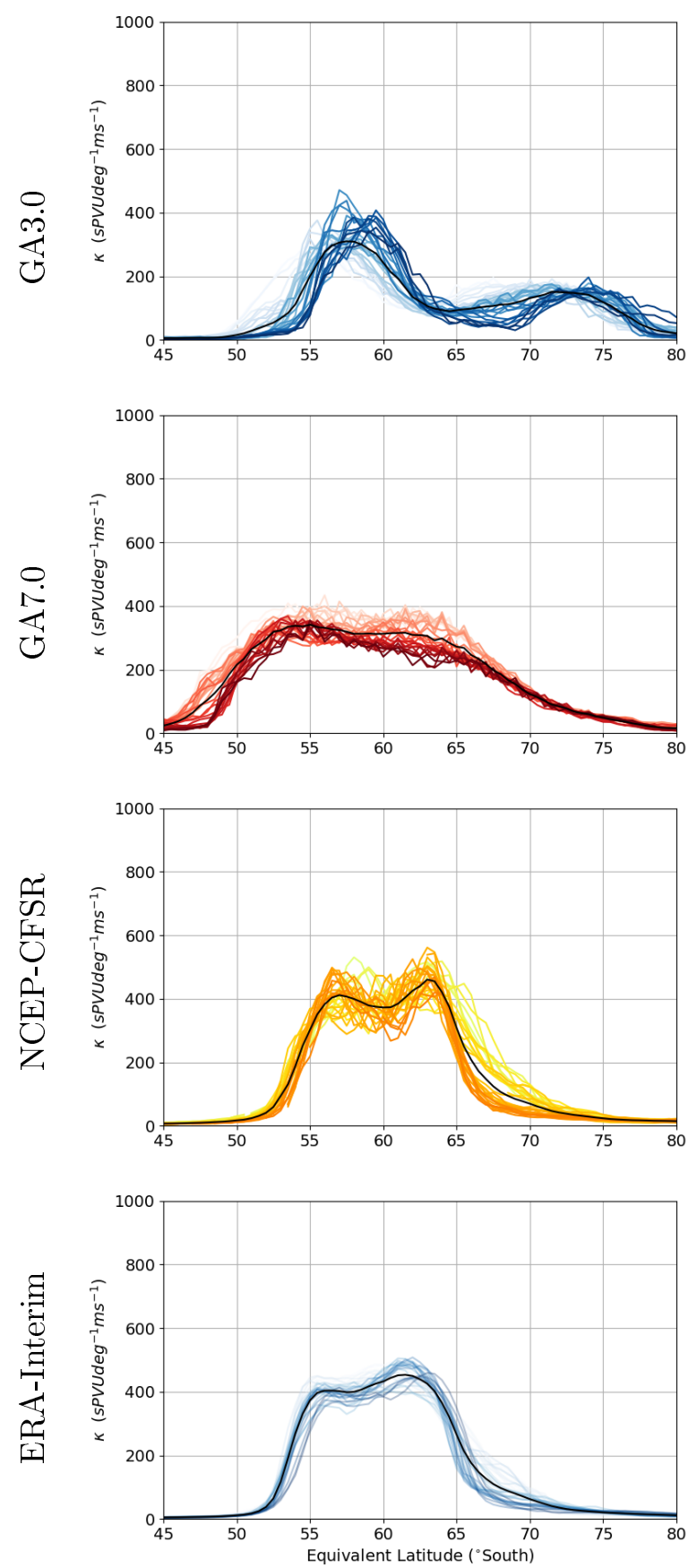

2008
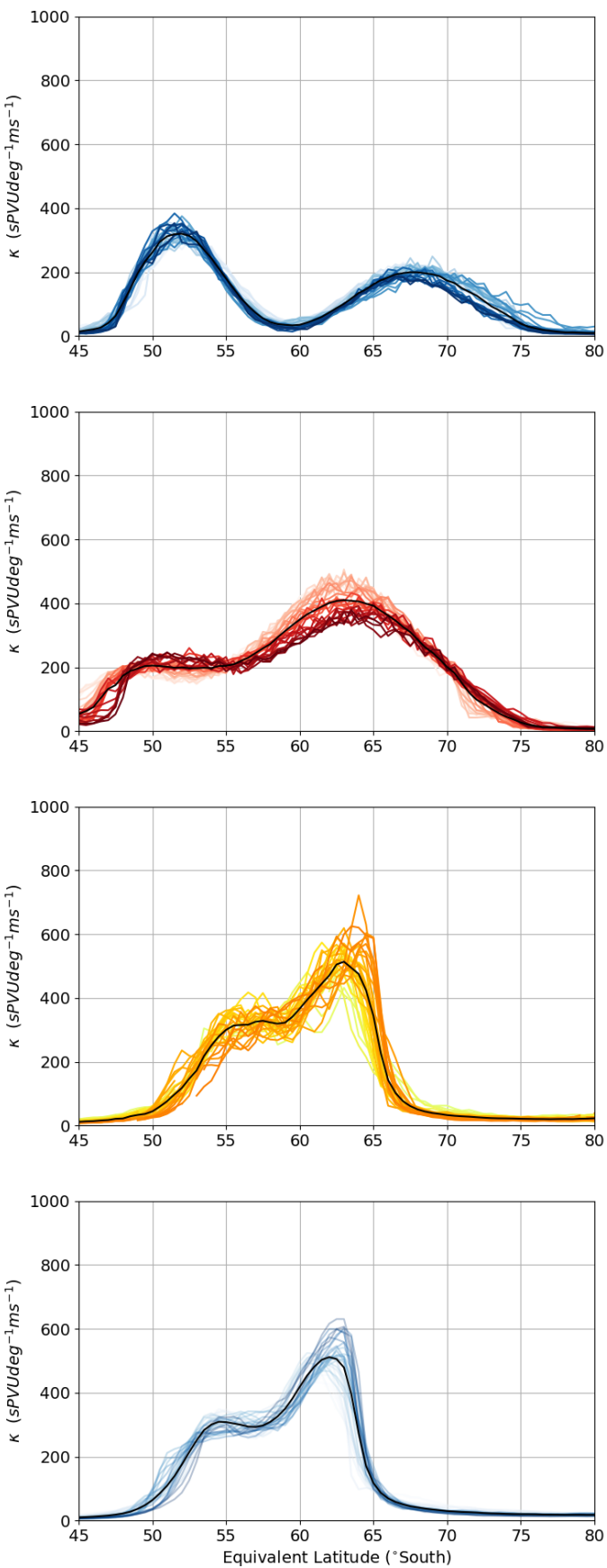

Figure 4.2: Daily (colours) and monthly (black lines) average $\kappa$ profiles by equivalent latitude for UM-GA3.0 (top), UM-GA7.0 (2nd row), NCEP-CFSR (3rd row), and ERA-Interim (bottom) at $550 \mathrm{~K}$. Plots are for September 1984 (left column) and September 2008 (right column). Lighter colours indicate the start of the month and darker colours the end of the month. 


\subsubsection{Time series analysis}

From zonal-mean values by equivalent latitude for each day, the maximum value for the variable of interest between equivalent latitudes of $45^{-} 85^{\circ} \mathrm{S}$ is first selected. Then the average of these daily maximums is calculated for the selected month, and for each year of the period.

It is important to note that the free-running UM simulations used here are not expected to simulate the year-to-year variability seen in the reanalyses, due to the chaotic nature of the climate and the dependence of each model simulation on the initial conditions (e.g., Brown et al. 2012). Such simulations are well suited to assessment of the internal variability of the climate system, but also allow for trends over multi-decadal simulations to be assessed. However, although trends may be apparent in reanalyses, these must be treated with caution because one feature of reanalyses is that both the quality and quantity of assimilated observations have increased over time, which may induce artificial trends (Screen and Simmonds, 2011). This is of particular importance for areas where continuous long-term observations are lacking, as is the case for polar regions (ibid). With such caveats in mind, trends in the annual time series for September mean maximums were evaluated using simple linear regression, with significance determined on the basis of p-values $(\leq 0.05)$ and detection of significance in trends at \pm 1 standard deviation $(1 \sigma)$.

\section{Annual}

The time series of the September mean maximum wind-speed is shown in Figure $4.3 \mathrm{a}$ for the $550 \mathrm{~K}$ level over the period 1983-2016. While GA7.0 compares favourably with the reanalyses, GA3.0 simulates much lower maximum wind-speeds. Interestingly, no significant trend is detected in windspeeds for either the NCEP-CFSR or the ERA-Interim reanalyses over the period used here (whereas a significant increase is found for the different period (1981-2009) examined in Chapter 5, section 5.3.3). It is possible that the lack of a trend here relates to the recovery of the ozone hole for the more recent years, with associated reductions in vortex wind-speed - however, this is purely speculative. GA3.0 showed no significant trend for maximum windspeed and is also significantly different from the other traces based on t-test results. A small but significant increasing trend was detected for maximum wind speed in GA7.0 $\left(0.09 \pm 0.04 \mathrm{~ms}^{-1}\right.$ per year over the period, with an Rsquared of 0.11 ).

For the September means of the meridional maxima in absolute PV at $550 \mathrm{~K}$ (Figure 4.3b), much lower absolute PV values are seen in GA3.0 com- 

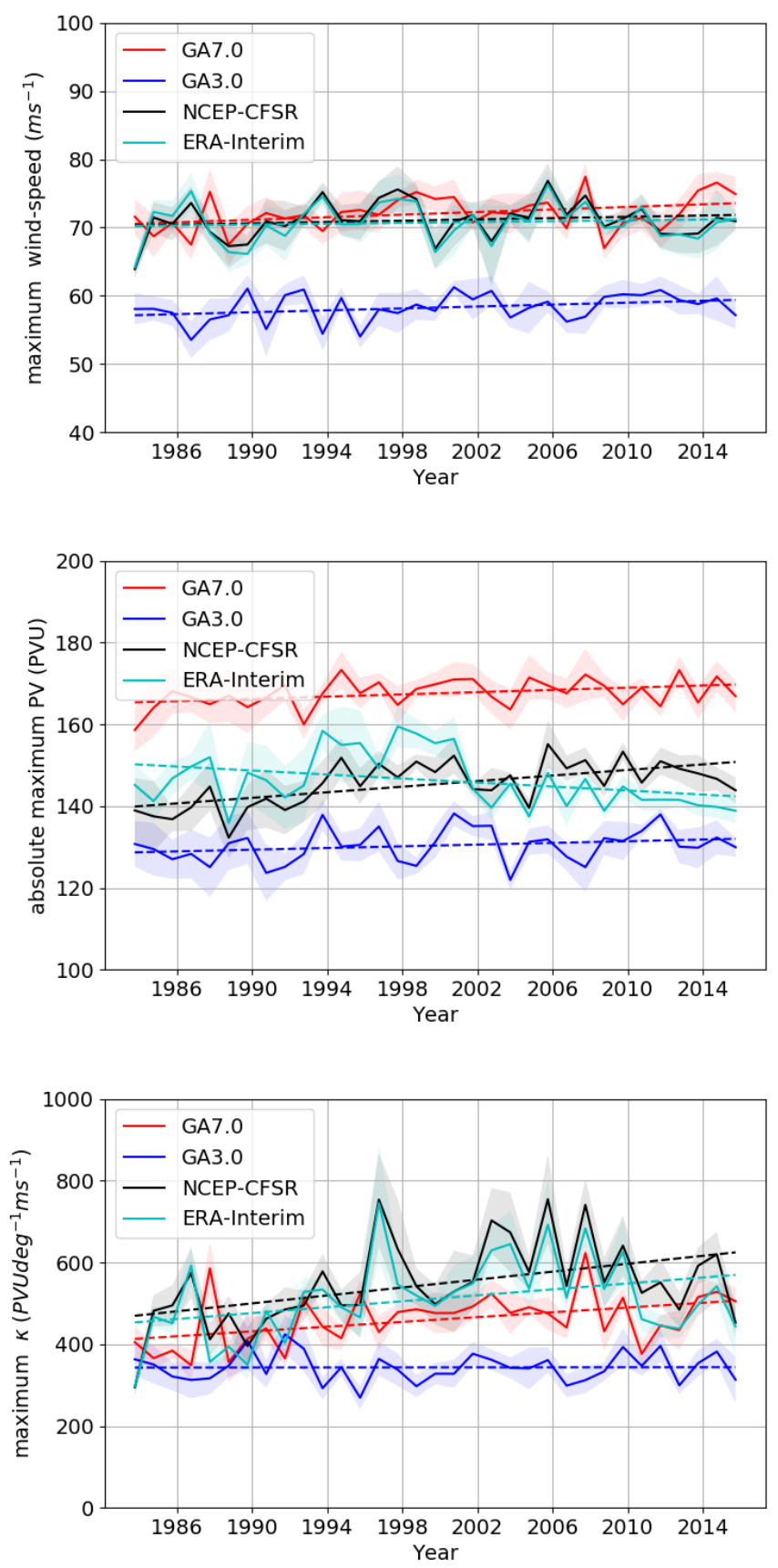

Figure 4.3: Time series of September average (a) meridional maximum windspeed, (b) meridional absolute maximum PV and (c) meridional maximum $\kappa$ values for GA3.0 (blue), GA7.0 (red), NCEP-CFSR (black), and ERAInterim (cyan) at the $550 \mathrm{~K}$ level (1983-2015). The maximum monthly average values are chosen from monthly average values calculated for $1^{\circ}$ equivalent latitude steps from $45-85^{\circ} \mathrm{S}$. The shaded areas denote the spread of values at $1 \sigma$ either side of the mean. 
pared with GA7.0 and both are statistically significantly different from the reanalyses. Significant trends are apparent in the reanalyses, but interestingly with opposite signs for each. PV increases over the period in NCEP-CFSR $(0.34 \pm 0.08$ PVU per year, with an R-squared of 0.42$)$, while there is a very weak but still significant decrease in ERA-Interim $(-0.22 \pm 0.10$ PVU per year, with an R-squared of 0.06). It is noted that over the period 1981-2009, PV showed an increasing trend in NCEP-CFSR, and no trend in ERA-Interim (Chapter 5). The GA7.0 configuration also shows a positive trend in PV $(0.14 \pm 0.06$ PVU per year, with an R-squared of 0.21$)$. No trend in maxima of absolute PV in September is apparent in GA3.0.

September mean meridional maximum $\kappa$ at $550 \mathrm{~K}$ (Figure $4.3 \mathrm{c}$ ) shows routinely higher values in the reanalyses. However, in the GA7.0 simulation these differences are not significantly different from the reanalyses, while in GA3.0 they are significantly different from the reanalyses. Significant positive trends are apparent in the reanalyses for both NCEP-CFSR $(4.8 \pm 1.7 \kappa \mathrm{U}$ per year over the period, with an R-squared of 0.24), and for ERA-Interim $(3.2 \pm 1.5 \kappa \mathrm{U}$ per year over the period, with an R-squared of 0.15$)$. A significant trend in maximum $\kappa$ for GA7.0 $(2.9 \pm 1.1 \kappa \mathrm{U}$ per year over the period, with an R-squared of 0.09 ) is found, while there is no trend in $\kappa$ for GA3.0.

\section{Pentads}

To assess both latitudinal and temporal change in the behaviour of $\kappa$ at the $550 \mathrm{~K}$ level, pentads (five-year averages) are compared between the two UM configurations and the two reanalyses (Figure 4.4). Each pentad is referred to here by the middle year of the five-year period (i.e., 1988, 1993, 1998, 2003, 2008, 2013 respectively). GA3.0 (Figure 4.4a) shows little change in the peak value, but a possible poleward shift in its location over time. GA7.0 (Figure $4.4 \mathrm{~b})$ shows very little change in the maximum $\kappa$ peaks over time, while both reanalyses(Figure 4.4c, d) show strikingly similar curves, and display maximum $\kappa$ values monotonically increasing from 1988 through to 2003 and thereafter reducing.

The blue lines in Figure 4.4 are almost comparable to pentad distributions from the older NCEP-NCAR reanalysis from Struthers et al. (2009), shown below (Figure 4.5 - their Figure 3f) against UMETRAC (Unified Model with Eulerian Transport and Chemistry). UMETRAC uses an older configuration of the UM core, based on UM version 4.5, which pre-dated the introduction of the New Dynamics formulation. This figure is almost comparable to Figure 4.4, but has two key differences: First, it is centered on 1997, while here 1998 is the middle year for the comparable pentad period. Second, Figure 4.5 shows curves for October, while the pentads compared in Figure 4.4 are 

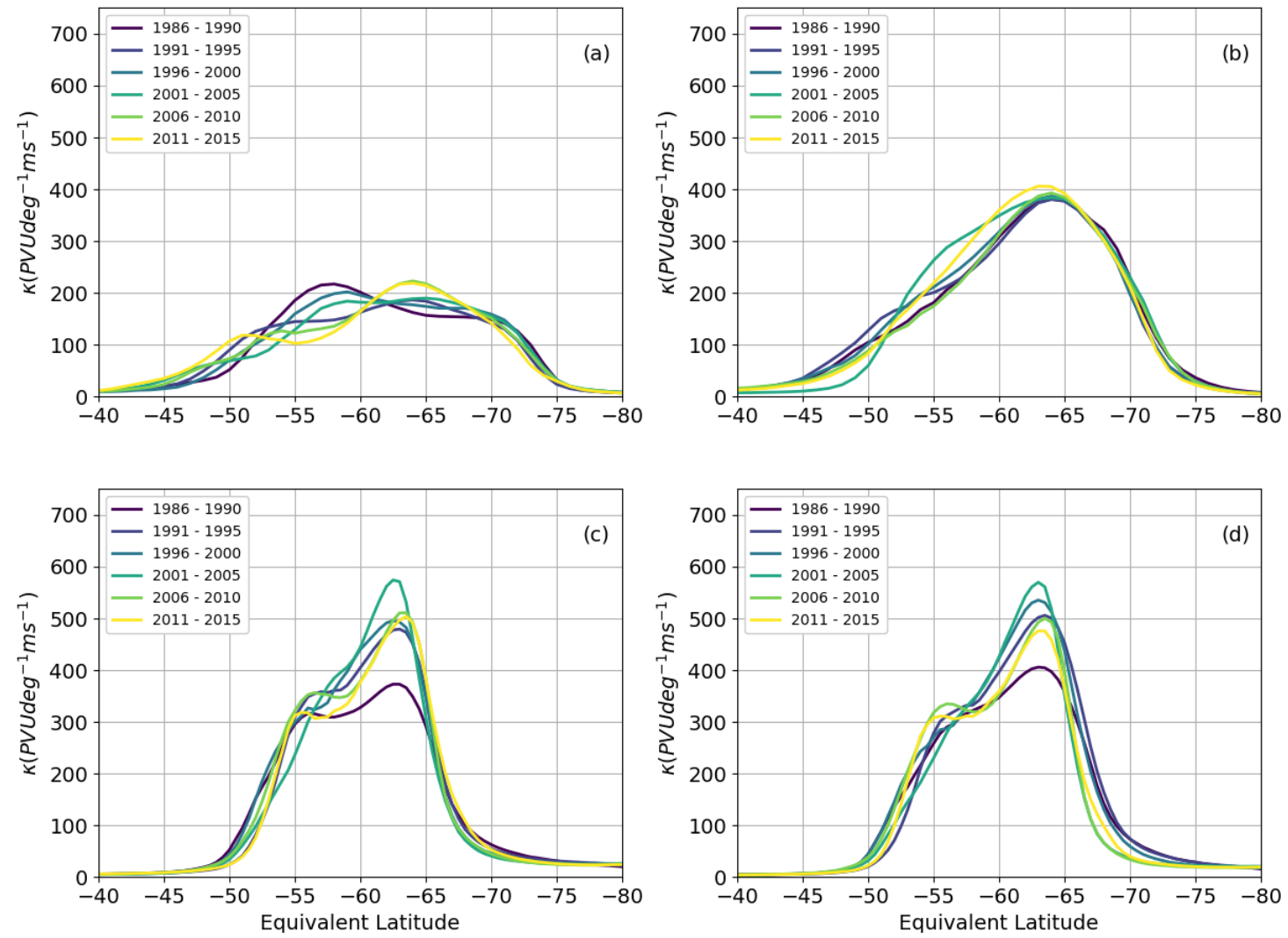

Figure 4.4: Pentads of $\kappa$ profiles by equivalent latitude at $550 \mathrm{~K}$, averaged for September for (a) GA3.0, (b) GA7.0, (c) NCEP-CFSR, (d) ERA-Interim. 


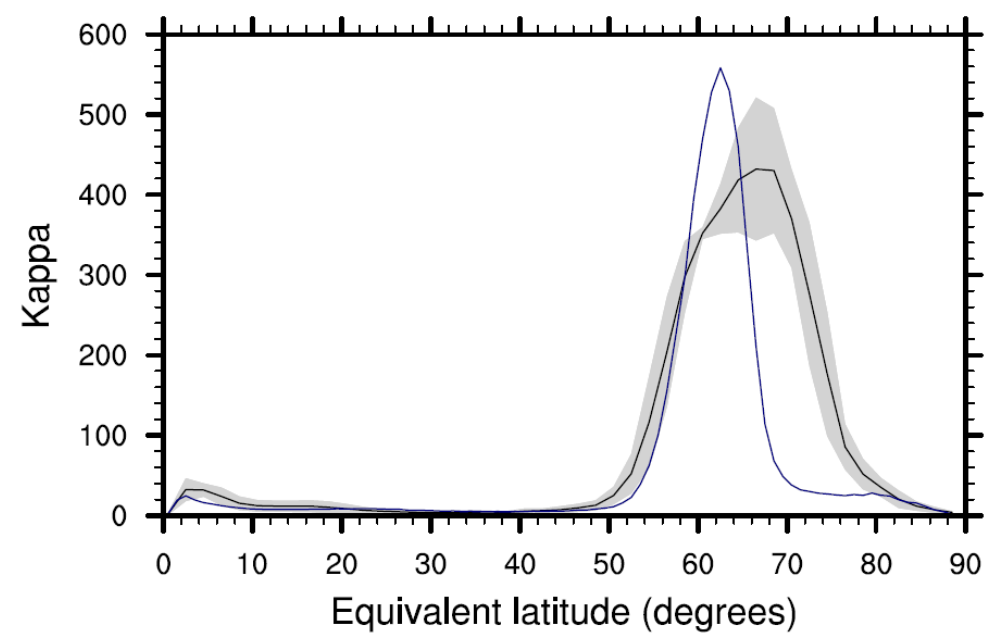

Figure 4.5: Five year (centred on 1997) average October $\kappa$ at $550 \mathrm{~K}$ from UMETRAC (black line, where shading indicates the $1 \sigma$ standard deviation about the mean) and NCEP-NCAR reanalysis (blue line). Source: Figure $3 \mathrm{f}$ in Struthers et al. (2009).

for September. In UMETRAC, the $\kappa$ distribution at $550 \mathrm{~K}$ during October 1995-1999 has FWHM that is about $8^{\circ}$ wider than in NCEP-NCAR reanalysis and extends poleward of NCEP-NCAR. The UMETRAC distribution has a lower maximum $\kappa$ located around $4^{\circ}$ south of that in NCEP-NCAR reanalyses. Their analysis showed a much more symmetrical $\kappa$ curve, while the updated reanalyses used here (using NCEP-CFSR and ERA-Interim, and more recent UM configurations) shows a more variable $\kappa$ distribution with bifurcated peak values and a more poleward primary peak. GA7.0 (Figure $4.4 \mathrm{~b}$ ) demonstrates a similar but more equatorward $\kappa$ distribution and NCEP-CFSR and ERA-Interim show a similar $\kappa$ distribution but with greater variability with regard to peak location and asymmetry of the $\kappa$ curve, than the NCEP-NCAR reanalysis. Though the $\kappa$ profiles in GA7.0 show a reduction in the poleward bias of the earlier UMETRAC configuration, the general shape of the $\kappa$ profile in GA7.0 more closely resembles that of UMETRAC than of GA3.0. Nonetheless these features demonstrate some of the improvements seen in more recent versions of the UM, as well as improvemens in the modern-era reanalyses, but these results also demonstrate that the poleward bias is a long-standing issue for climate modelling. It is noted that the NCEP-NCAR reanalysis has since been shown to provide poor representation of the stratosphere due to its very low model top, few model levels and its outdated assimilation approaches (Manney et al., 2003).

Previous authors (e.g., Thompson and Solomon 2002; Bodeker et al. 2002; 
Thompson et al. 2011) have shown that the ACV barrier has strengthened over time in conjunction with a trend toward a more frequent positive phase of the SAM (Thompson and Wallace, 2000), attributed to stratospheric ozone depletion. The reduction in the strength of the barrier in September at the $550 \mathrm{~K}$ level, seen here for the reanalyses from about 2007 (Figure 4.3), is consistent with a recovering ozone hole.

\subsubsection{Vertical profiles}

Using maximum monthly zonal-means across all SH equivalent latitudes between $45-85^{\circ} \mathrm{S}$, the September climatology (1983-2015) is plotted against isentropic level in Figure 4.6. The average maximum value of wind-speed in September (Figure 4.6a) shows that GA7.0 compares well to the reanalyses maximum wind-speeds at lower levels (up to around $650 \mathrm{~K}$ ). In both UM configurations the wind-speed increases up to around $1200 \mathrm{~K}$, and does not capture the reduction in wind-speed at higher levels seen in the reanalyses (above about $850 \mathrm{~K}$ ). These strong wind-speeds at the highest model levels may be associated with the parameterization scheme used for gravity wave drag in the UM configurations (i.e., insufficient GWD to reduce upper level winds sufficiently) - this is discussed in further detail below.

The vertical profile of maximum absolute standardised PV (Figure 4.6b) shows GA7.0 generally having the largest values at the lowest levels (from $400 \mathrm{~K}$ up to about $850 \mathrm{~K}$ ), corresponding roughly to the extent of the ozone layer. Both the maximum PV gradient and maximum $\kappa$ (Figure $4.6 \mathrm{c}$ and 4.6d) reach their peak for GA7.0 at about $750 \mathrm{~K}$, with $\kappa$ well above the reanalyses. The stronger barrier in GA7.0 continues above this level in $\kappa$, consistent with the higher wind-speeds.

Monthly climatological mean (1983-2015) cross-sections of PV gradient against equivalent latitude and potential temperature are shown for ERAInterim, NCEP-CFSR, GA7.0 and GA3.0 for the austral winter and spring months July, August and September in Figure 4.7. In these figures, windspeed contours are shown in $5 \mathrm{~ms}^{-1}$ steps and PV is displayed using standardised PV. The figures represent the two components of $\kappa$, namely $\partial P V / \partial \phi_{e}$ and wind-speed.

Firstly, comparing the two reanalyses, a wider and stronger vortex boundary region exists in ERA-Interim (Figure $4.7 \mathrm{a}-\mathrm{c}$ ) than in NCEP-CFSR (Figure $4.7 \mathrm{~d}-\mathrm{f})$. This region extends both further poleward in ERA-Interim $\left(\phi_{e}\right.$ range of approximately $\left.62-67^{\circ} \mathrm{S}\right)$ than in NCEP-CFSR $\left(62-65^{\circ} \mathrm{S}\right)$, and extends over a greater range of potential temperatures (approximately 480$650 \mathrm{~K}$ in ERA-Interim, compared with $520-600 \mathrm{~K}$ in NCEP-CFSR). The PV gradient in these regions is up to $30 \%$ stronger in ERA-Interim, though PV 

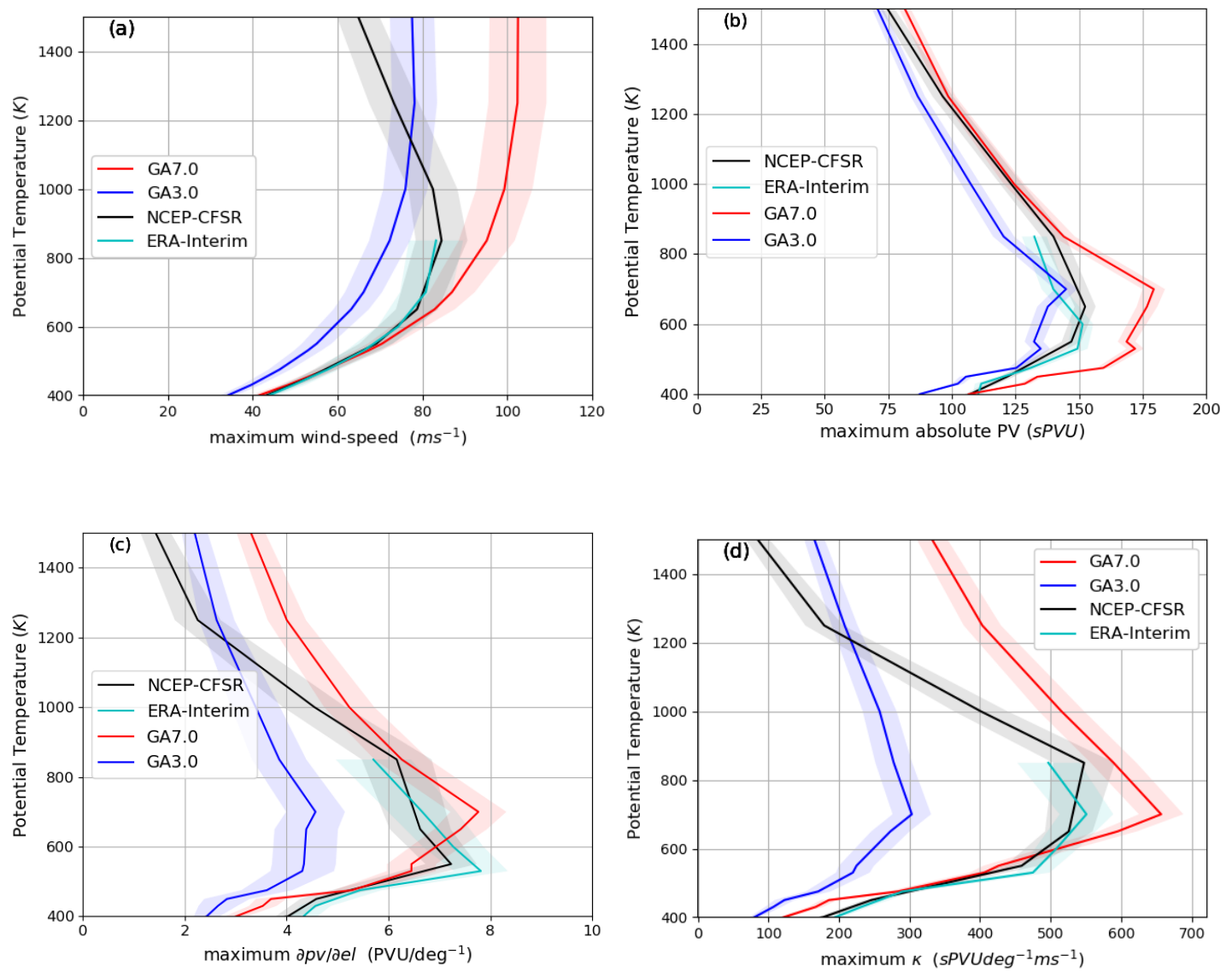

Figure 4.6: Climatological vertical profiles between $400 \mathrm{~K}$ and $1500 \mathrm{~K}$ for September over the period 1983-2015 for (a) maximum monthly average wind-speed; (b) absolute maximum monthly average $s P V$; (c) maximum monthly average $s P V$ gradient; (d) maximum monthly average $s \kappa$. Plots are shown for GA3.0 (blue), GA7.0 (red), NCEP-CFSR (black) and ERA-Interim (cyan), where the shaded areas denote the spread of values at $1 \sigma$ either side of the mean. The maximum monthly average values are chosen from monthly averages calculated for $1^{\circ}$ equivalent latitude steps from $45-85^{\circ} \mathrm{S}$. 
July
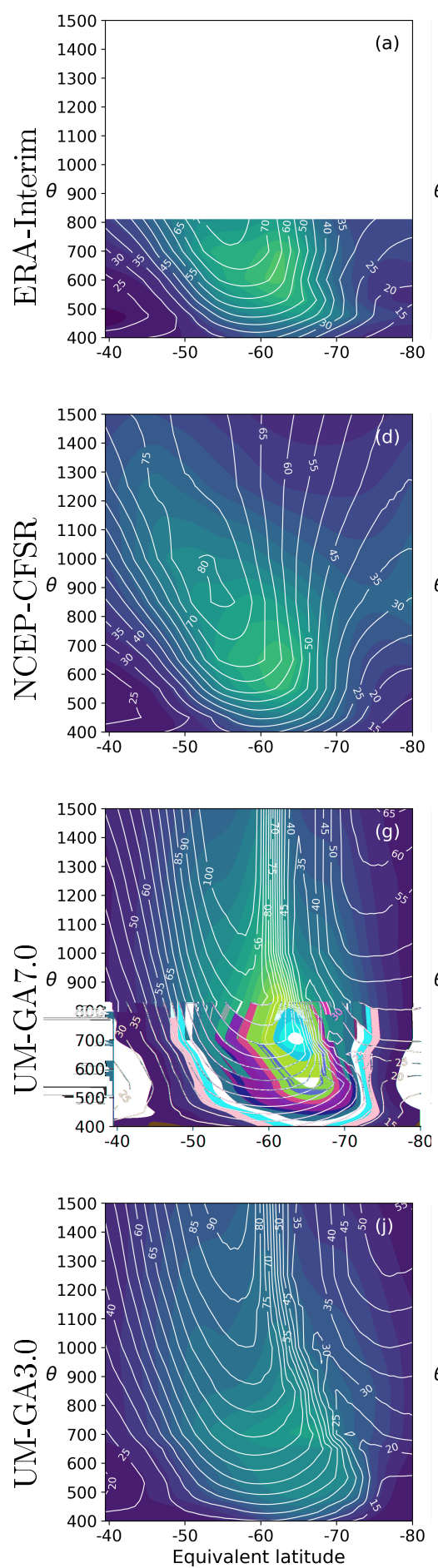

August
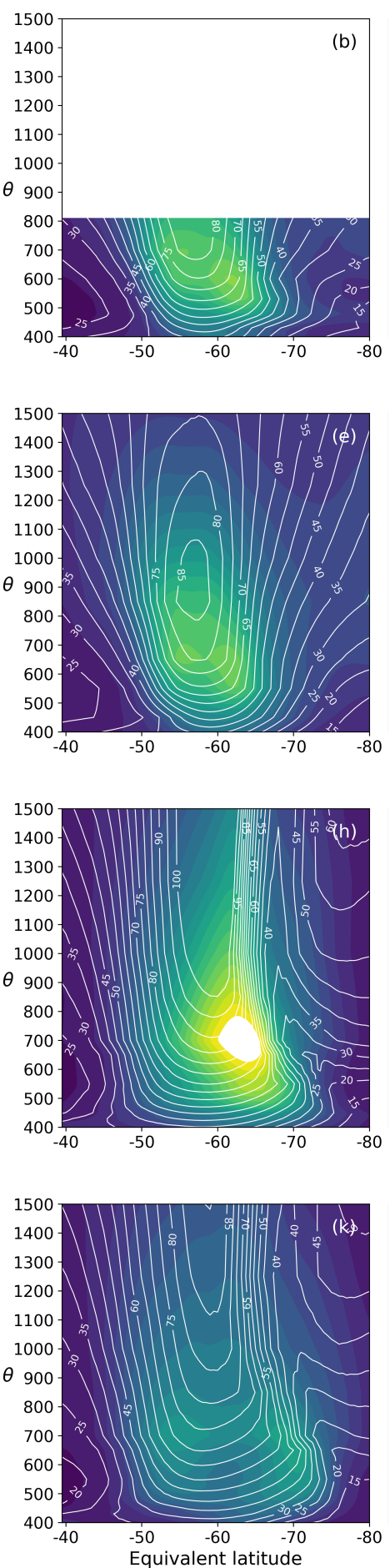

September
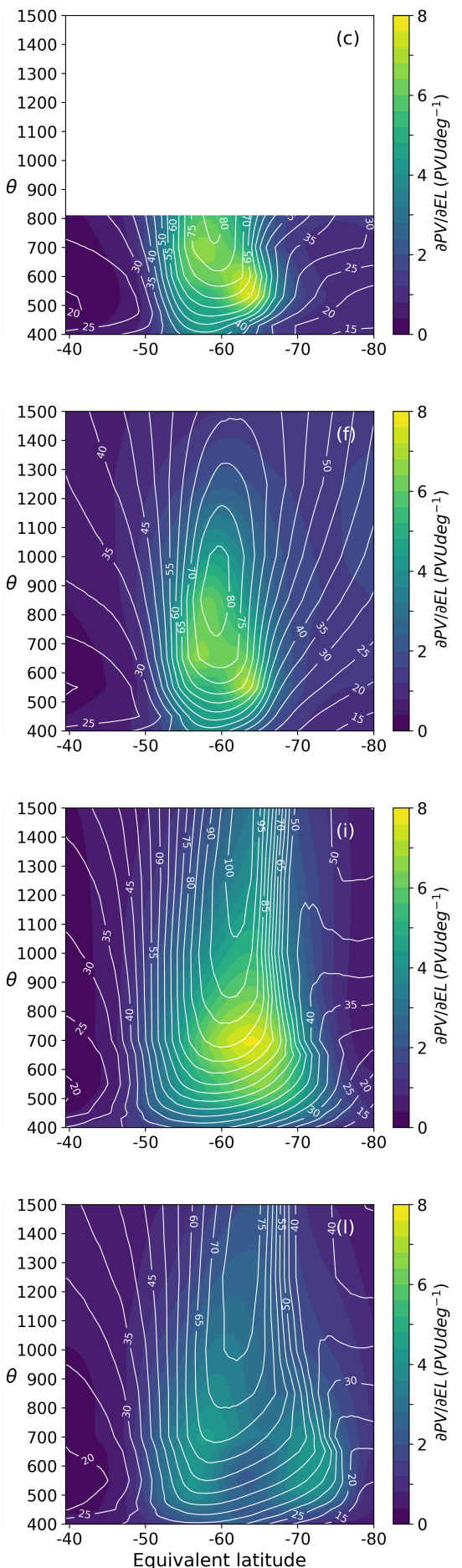

Figure 4.7: Climatological mean (1983-2015) structure of $\partial P V / \partial \phi_{e}$ by equivalent latitude (colours; $s P V U d e g^{-1}$, where white shading represents PV gradients of $>8.0$ ) and wind-speed (white lines; in $5 \mathrm{~m} \mathrm{~s}^{-1}$ steps) at levels between $400 \mathrm{~K}$ and $1500 \mathrm{~K}$ for July to September in: ERA-Interim (a)-(c), NCEP-CFSR (d)-(f), GA7.0 (g)-(i), and GA3.0 (j)-(l). 
gradients are typically within $\pm 10 \%$ of each other between the two reanalyses in other areas of the lower and middle-stratosphere. The reanalyses show the evolution of the ACV aligned with peak wind-speeds, and strengthening from July to September. A bifurcated vortex structure is seen in August and September as two zones of high PV gradient. In September, these are consistent with the $\kappa$ peak and 'shoulder' seen in Figure $4.1 \mathrm{~d}$ at $550 \mathrm{~K}$ at $63^{\circ} \mathrm{S}$ and $55^{\circ} \mathrm{S}$ respectively.

In the UM GA7.0 simulation, a consistently wider vortex barrier is observed compared with the reanalysis, with much higher PV gradients apparent at around $700 \mathrm{~K}$ and $63^{\circ} \mathrm{S}$ between July and September. This is particularly evident in August, where the PV gradient exceeds $8.0\left(P V U d e g^{-1}\right)$ from $60-67^{\circ} \mathrm{S}$ and $600-800 \mathrm{~K}$. The maximum PV gradient is less well aligned with maximum wind-speeds than seen in the reanalysis. The GA3.0 simulation displays both lower PV gradient and lower wind-speeds, compared with both GA7.0 and the reanalyses.

Overall, ERA-Interim appears to be slightly more similar to GA7.0 in its representation of the vortex barrier than NCEP-CFSR - at least at levels up to about $650 \mathrm{~K}$. Above around $1000 \mathrm{~K}$ the barrier in GA7.0 is much too strong. Improvements in the UM for GA7.0 have resulted in vastly better simulation of the vortex barrier at levels below $650 \mathrm{~K}$.

At levels above about $600 \mathrm{~K}$, from July to September, the winds simulated in the GA7.0 model configuration (Figure 4.7g-i) indicate two stratospheric jets - at $60^{\circ} \mathrm{S}$ and $75^{\circ} \mathrm{S}$ equivalent latitude, with very steep wind-speed gradients between the two wind-speed maxima. A similar, though lower strength wind-speed pattern, is seen in the GA3.0 model configuration (Figure $4.7 \mathrm{j}-1$ ). The second jet, located at $75^{\circ} \mathrm{S}$ equivalent latitude, is an erroneous artefact of mapping wind fields to equivalent latitude when PV does not monotonically increase toward the pole, and most likely results from methods inherent to the dynamical core used in the UM. Such an artefact is missing from the reanalysis since any aberrant behaviour in the reanalysis would be quickly suppressed as measurements are assimilated.

The climatological mean (1983-2015) cross-sections of $\kappa$ against equivalent latitude and potential temperature are shown in Figure 4.8. Consistent with the patterns observed for Figure 4.7, the cross-sections show a generally stronger barrier in GA7.0 (Figure $4.8 \mathrm{~g}-\mathrm{i}$ ) and a weaker barrier in GA3.0 (Figure $4.8 \mathrm{j}-1)$, compared with reanalyses, with both UM configurations displaying the now familiar wider poleward barrier than that seen in the reanalyses. ERA-Interim (Figure 4.8a-c) indicates a slightly stronger and wider barrier than NCEP-CFSR (Figure 4.8d-f). Better simulation of $\kappa$ at lower model levels in GA7.0 is apparent in each month. 
94 CHAPTER 4. THE VORTEX BARRIER IN THE UNIFIED MODEL

July
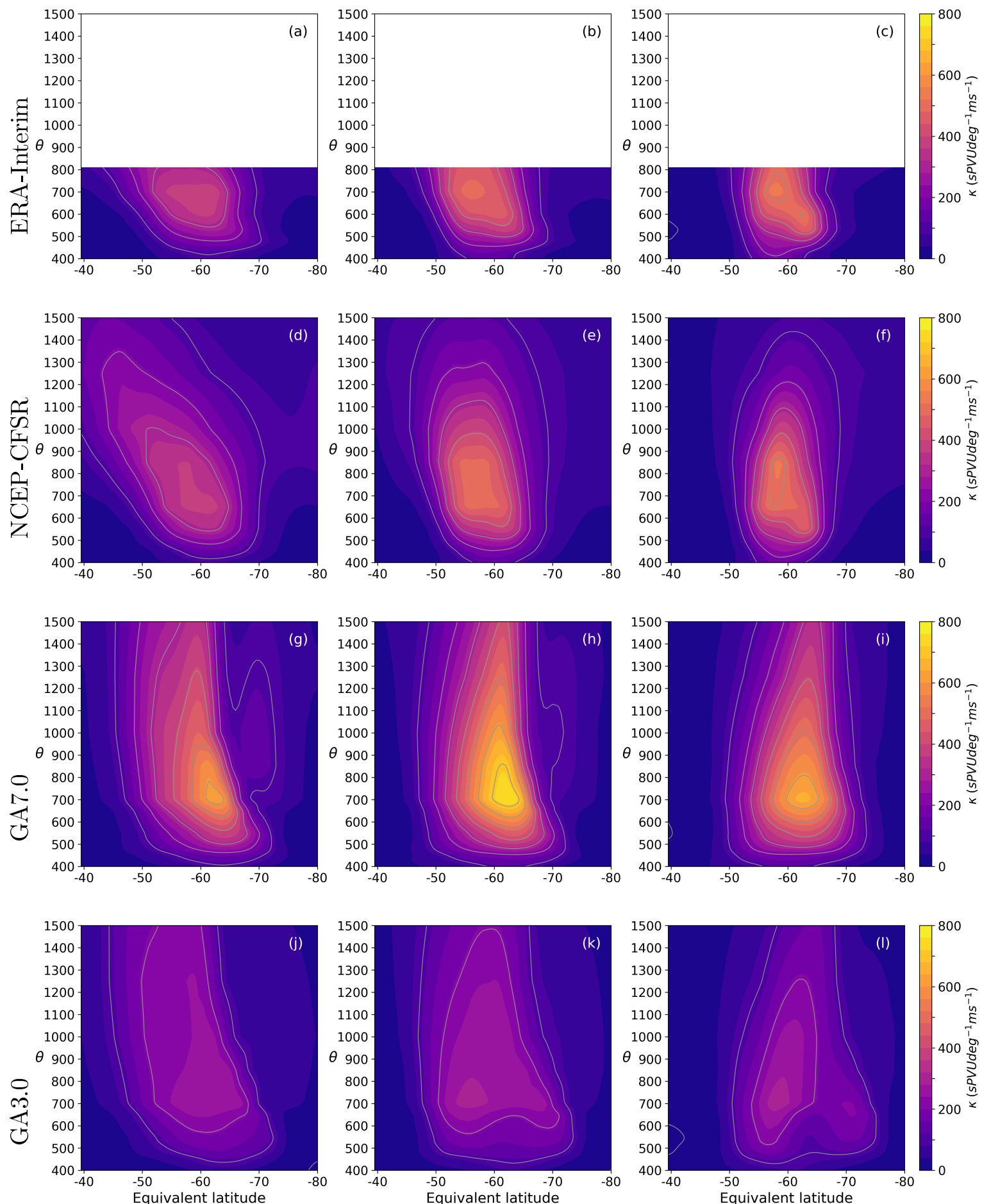

Figure 4.8: Climatological mean (1983-2015) structure of standardised $\kappa$ (colours; $\mathrm{s} \kappa \mathrm{U}$, and grey contour lines at $100 \mathrm{~s} \kappa \mathrm{U}$ steps) at levels between $400 \mathrm{~K}$ and $1500 \mathrm{~K}$ for July to September in: ERA-Interim (a)-(c), NCEPCFSR (d)-(f), GA7.0 (g)-(i), and GA3.0 (j)-(l). 
Bifurcation in $\kappa$ appears in GA7.0 in July above about $800 \mathrm{~K}$, and at around $550 \mathrm{~K}$ in GA3.0 in September. However, as noted earlier, this bifurcation is likely to result from a non-monotonic increase in PV toward the pole in the model. When PV is not monotonically increasing toward the pole, the $\kappa$ profile can vary considerably in strength, shape and extent based on the location of the wind-jet.

\subsection{Discussion and conclusions}

Representation of the ACV transport barrier was compared using two configurations of the UM and two modern-era reanalyses. The GA7.0 model configuration appears to better simulate the dynamical isolation of the ACV in the lower and middle stratosphere, up to isentropic levels of around $600 \mathrm{~K}$, while the GA3.0 configuration provides a better representation of $\kappa$ in the stratosphere above this level. However, neither UM configuration simulates the same degree of dynamical isolation suggested by reanalyses, which have much narrower meridional bands of high wind-speed and varying PV gradients, with a stronger barrier in GA7.0 and a weaker barrier in GA3.0. Both UM configurations produce a wider and more poleward band of high windspeed, steep PV gradients and meridional impermeability when compared with the reanalyses. For the reanalyses, ERA-Interim simulates a slightly stronger barrier than NCEP-CFSR, which errs more toward the results seen here for GA7.0, suggesting that it may be a more suitable comparison with model results in future analysis work.

The generally improved performance in GA7.0 relative to GA3.0 shown here, at least in the lower and mid-stratosphere, is likely due in part to the more stable numerical scheme used in the ENDGame dynamics, which has reduced wave damping, intensified cyclones, fronts and jets, and improved overall model accuracy (Walters et al., 2017). Several other aspects of the UM physics have been substantially developed since GA3.0, including radiation, cloud, precipitation, convection and gravity wave drag (Walters et al. 2017; Walters et al. 2018). In GA7.0 the parameterization of non-orographic gravity wave drag at sub-grid scales is tuned to the model resolution (N96) to better reproduce the observed QBO period in the equatorial stratosphere. This scheme can also affect the strength and tilt with height of the winter stratospheric jet (Scaife et al., 2002). GA7.0 also uses an updated scheme to represent sub-grid orographic gravity waves, which have been shown to influence the strength of the ACV in the Whole Atmosphere Community Climate Model (Garcia et al., 2017). Developments such as these are likely to have contributed to the differing representations of the ACV in GA7.0 and 
GA3.0, but their combined use here makes it difficult to quantify the effects of individual changes to the model physics.

Poleward displacement of the inner vortex edge, often seen in CCMs (e.g., Morgenstern et al. 2009) may impede their ability to accurately simulate the ozone hole area, radiative forcing and temperature gradients in the Antarctic stratosphere, which would in turn feed back onto the model dynamics. Struthers et al. (2009) found that the poleward edge of the ACV was located significantly too far south in three different CCMs, including UMETRAC (section 4.2.3). By measuring the area on the $50 \mathrm{hPa}$ pressure surface where temperatures are below $195 \mathrm{~K}$, Morgenstern et al. (2009) found that the size of the region contained within the ACV was underestimated by the UK Chemistry and Aerosols (UKCA) model coupled to the UM with New Dynamics. This suggests that the zone of high meridional impermeability may also have extended too far south in that configuration of the model. In the HadGEM3ES chemistry-climate model, based on Global Atmosphere 4.0 (GA4.0) with New Dynamics, it has been suggested that the poleward displacement of the ACV might be related to the attenuation of non-orographic gravity waves in the upper stratosphere (Hardiman et al., 2017).

The high $\kappa$ values at middle and high isentropic levels for GA7.0 (Figure $4.8 \mathrm{~d}-\mathrm{f}$ ) raises questions about the performance of this UM configuration at these levels. Due to the way in which gravity waves are propagated upward through the atmosphere, it is possible that this difference at the highest levels of GA7.0 relates to the newer parameterization of gravity waves in that configuration of the UM. Reduced damping within the ENDGame dynamical core would be expected to lead to higher wind-speeds and greater vorticity in the upper stratosphere, however further research would be required to verify that these factors are responsible for the differences in representation of the $\mathrm{ACV}$ seen here between model results and reanalyses.

Prescribing ozone in the stratosphere of a GCM can result in significant spatial and temporal mismatches between the internal model dynamics and the radiative forcing field from ozone (e.g., Crook et al. 2008; Neely et al. 2014), regardless of the temporal or spatial resolution of the prescribed ozone field. For example, extra-vortex ozone may end up being specified inside the vortex, or lower stratospheric ozone may end up being prescribed in the upper troposphere when the model tropopause is high. It is conceivable that the resultant mismatch between the ozone short-wave radiative forcing and the dynamically driven model temperature field results in a smoothing of temperature and PV gradients and consequent flattening of meridional $\kappa$ profiles in the UM that are seen here.

Coupled chemistry-climate models (CCMs), in which ozone fields are sim- 
ulated and are coupled to the model dynamics, are expected to better simulate the impermeability of the ACV compared to models in which ozone fields are prescribed. However, interactively simulating stratospheric ozone is computationally expensive - around $60 \%$ of the climate models that contributed to the IPCC $5^{\text {th }}$ Assessment Report relied on monthly-mean zonal-mean vertically resolved data sets to prescribe ozone concentrations (Eyring et al., 2013). Furthermore, even models with interactive chemistry exhibit varying performance in their simulation of the $\mathrm{ACV}$, which tends to be more poorly represented than its counterpart in the NH. In a range of CCMs, the Antarctic polar night jet is too strong in the upper stratosphere and does not tilt sufficiently toward the equator with increasing height (Butchart et al., 2011). This same pattern is seen here for the UM, where the equatorward tilt is greater in the reanalyses, particularly in July (left column of Figure 4.8).

Ideally, this analysis would have led to robust conclusions regarding the processes responsible for improved performance in GA7.0 - as well as its overestimation of $\kappa$. Here, however, many factors have changed between the GA3.0 and GA7.0 model simulations. In addition, many components of the model have been optimized to minimise biases, which may have introduced compensating errors. With so many factors changing, it is not possible to categorically pinpoint the driver(s) of the improved performance in GA7.0, or the reasons for its overestimation of $\kappa$. To unpack the various elements responsible for the results observed here would require additional model simulations that isolate a small number of processes of interest. For example, simulations could be run for GA7.0 that examine the relative importance of differing model physics to demonstrate which model components result in improved representation of $\kappa$. In this project, resource constraints meant that it was not possible to carry out additional simulations. However, ongoing efforts, such as this one, will be required to validate atmospheric prediction systems to assess progress and identify features that can be improved.

Future research may benefit from similar assessment in the performance of a range of of other GCMs and CCMs, to test to what extent they are modelspecific. The higher horizontal resolution output fields from the reanalysis compared to the UM may also be a factor in the differences seen in the results here - while the impact of different resolutions was not examined here, it may be an area for additional research focus.

The analysis undertaken here does not directly address the likely stratospheric and climatic changes resulting from future ozone recovery and anthropogenic climate change. However, improving the representation of the dynamics of the ACV in global climate models is a first important step toward 
reducing related uncertainties in projections of climate change. Similarly, accurate representation of zonal and temporal variations in ozone concentrations may improve the ability of models to simulate the effects of ozone changes on the climate system (e.g., Crook et al. 2008; Dennison et al. 2017). 


\section{Chapter 5}

\section{The vortex barrier in the SOCOL model}

One of the conjectures outlined in Chapter 4 was that the weaker dynamical isolation simulated by the UM may result, at least in part, from those model simulations being performed using prescribed ozone fields rather than having ozone simulated interactively in the model. In that research it was not possible to conduct a wide range of simulations in the UM with different interactive or prescribed ozone fields due to resource limitations. Prescribing ozone may lead to misalignment of the three-dimensional stratospheric ozone radiative forcing field and the internal model dynamical fields, thus flattening the temperature, potential vorticity, ozone and other trace gas gradients across the vortex edge. Here, representation of the ACV using prescribed ozone in a chemistry climate model is compared with model simulations using interactive ozone chemistry, and with reanalyses.

\subsection{Ozone in climate models}

Ideally, climate models would include an interactive stratospheric chemistry scheme to capture the two-way coupling between ozone and dynamics. However, since CCMs are computationally expensive to run, the current generation of GCMs tend to prescribe either zonal-mean, monthly-mean, or both zonal and monthly-mean ozone climatology to reduce the computational burden. Previous studies have shown that modelling of SH circulation is adversely affected by the use of either spatially or temporally averaged ozone climatologies (e.g., Crook et al. 2008; Waugh et al. 2009; Gillett et al. 2009; Neely et al. 2014). In particular, the polar vortex is found to be warmer and weaker - and thus less isolated - when ozone climatologies are prescribed 
(with either temporal or spatial averaging) - compared to when interactive stratospheric ozone chemistry is used (Waugh et al., 2009). This is thought to be due to the spatial smoothing resulting in either increased upward flux of wave activity (Crook et al., 2008), or damping of wave propagation (e.g., Gabriel et al. 2007; Nathan and Cordero 2007). This has led to the recommendation that, in the absence of interactive stratospheric chemistry, a zonally asymmetric ozone climatology concentration should be used, as opposed to the more common zonally averaged ozone climatology (Gillett et al., 2009).

While previous studies have evaluated the use of prescribed monthlymean zonal-mean ozone, they have not specifically investigated the impact on representation of the vortex transport barrier. In the case of the work of Gillett et al. (2009), the focus was on temperature responses to the zonal ozone asymmetry. Here, changes in the fidelity of the ACV based on meridional impermeability are explored when prescribing ozone using different approaches. Comparisons are made between reanalyses and output from several simulations using the same CCM. The simulations include one with interactive ozone chemistry, a second using a prescribed 3-D monthly-mean ozone field, and a third with a prescribed 2-D monthly-mean zonal-mean ozone field. The simulations undertaken here using prescribed ozone fields use a two member ensemble to assess the impact of internal variability against forced changes in those model configurations. This Chapter assesses these model simulations using the SOCOLv3 (Solar Climate Ozone Links) version 3 chemistry climate model (CCM). A paper outlining the results of this research was submitted for publication in November 2018 (Cameron et al., sbmt), with reviewer responses received and amendments underway. This chapter is based on that submission.

\subsection{Methods}

\subsubsection{The SOCOL model}

SOCOL was first released in 2005 as a Swiss-German collaboration (Egorova et al., 2005), using the dynamical core of the fifth generation of the middleatmosphere general circulation model MA-ECHAM (Middle-Atmosphere European Centre/HAMburg climate model) based on a flux-form transport scheme (Roeckner et al. 2003; Stenke et al. 2013). The model is able to simulate the principal feedback mechanisms that occur between radiative, photochemical, dynamical and advective processes. Detailed descriptions of the SOCOL model are found in Stenke et al. (2013) and Revell et al. (2015). 
Here, SOCOLv3 is run at the T42 horizontal resolution $\left(2.8^{\circ}\right.$ latitude $\times 2.8^{\circ}$ longitude). Boundary conditions are those recommended for the Chemistry-Climate Model Initiative (CCMI-1) reference simulation REF-C1 - a free-running simulation of the recent past, in which boundary conditions follow observations as closely as possible (Morgenstern et al., 2017). Sea surface temperatures and sea ice concentrations are sourced from the UK Met Office Hadley Centre (Rayner et al., 2003), while the surface mixing ratios of ODSs are sourced from the WMO A1 scenario (WMO, 2010). GHG concentrations are based on observations until 2005 and Representative Concentration Pathway (RCP) 8.5 thereafter (Riahi et al., 2011). For SOCOLv3, model dynamics are calculated at 15 minute time-steps, while the radiation scheme and model chemistry is called every two hours.

Access to enable simulations using the SOCOLv3 model was made available through collaborators at ETH (Swiss Federal Institute of Technology) in Zurich and Bodeker Scientific, New Zealand. Simulations were undertaken by Andrea Stenke at ETH with the assistance of Laura Revell (Bodeker Scientific and University of Canterbury).

\subsubsection{Model simulations}

The SOCOLv3 model is used here to examine vortex impermeability, where simulations based on the use of interactive ozone, and simulations using either 2-D or 3-D prescribed ozone, are compared with reanalyses. First, a SOCOLv3 reference model simulation, with fully interactive (INT) ozone chemistry is run for the period 1981-2009. Daily ozone fields output from the INT simulation were used to produce time-varying 3-dimensional (i.e., latitude, longitude, altitude) monthly-mean ozone fields (MM3D) and 2-dimensional (i.e., latitude, altitude) monthly-mean zonal-mean (MMZM) ozone fields.

Sensitivity simulations are then run, using the same initial conditions as used for INT, with the first sensitivity simulation driven by the prescribed MM3D ozone fields, and the second sensitivity simulation driven by the prescribed MMZM ozone fields. To establish the degree to which the internal variability of the SOCOLv3 model would affect the resulting simulations, an initial condition ensemble of two members was constructed for each of the sensitivity simulations, based on a slightly perturbed $\mathrm{CO}_{2}$ concentration for the first month of each simulation. The effect of monthly and zonal averaging of prescribed ozone on the strength of the ACV is examined through the SOCOLv3 output compared against the NCEP-CFSR and ERA-Interim reanalyses.

Model simulations were undertaken for 39 vertical levels ranging from $1000 \mathrm{hPa}$ to $0.01 \mathrm{hPa}$ (surface to $\sim 80 \mathrm{~km}$ altitude). In this research, daily 
mean temperature, zonal and meridional wind components, and ozone concentrations are calculated for 31 pressure levels (surface to $0.1 \mathrm{hPa}$ or $\sim 60 \mathrm{~km}$ ) from the average of four instantaneous values at 6 hourly intervals. Model output was used to calculate PV at each grid-point. Data were vertically linearly interpolated onto isentropic surfaces between $475 \mathrm{~K}$ and $1500 \mathrm{~K}$ for comparison with reanalysis. The isentropic levels used here were $475 \mathrm{~K}, 530 \mathrm{~K}$, $550 \mathrm{~K}, 650 \mathrm{~K}, 700 \mathrm{~K}, 850 \mathrm{~K}, 1000 \mathrm{~K}, 1250 \mathrm{~K}$ and $1500 \mathrm{~K}$. As for Chapter 4 the ERA-Interim reanalysis data used here only extends up to the $850 \mathrm{~K}$ level.

\subsubsection{Analysis}

The strength of the vortex barrier is calculated as for Chapter 4 using meridional impermeability $(\kappa)$, with the same focus on the meridional maximum values of $\kappa$ which occur around the location of the steepest PV gradient and maximum wind-speed across the vortex barrier. For each day, at each isentropic level, the equivalent latitude $\left(\phi_{e}\right)$ is calculated from PV fields. Mean wind-speed, PV and $\kappa$ are calculated for each $\phi_{e}$. Finally, monthly means of the $\phi_{e}$ meridional profiles are calculated for each simulation, and calendar month means over the 29-year time period calculated to obtain a climatology. The $550 \mathrm{~K}$ level was selected since it is the analysis level closest to the level of peak ozone concentration in the stratosphere, while September is typically the month of greatest ozone loss.

\subsection{Results}

\subsubsection{Climatology}

September monthly-mean climatological profiles (1981-2009) by equivalent latitude are shown in Figure 5.1, for wind-speed, PV, PV gradient, and $\kappa$ on the $550 \mathrm{~K}$ potential temperature $(\Theta)$ level. The profiles are compared between ERA-Interim, NCEP-CFSR and the five SOCOLv3 simulations. All of the SOCOLv3 simulations show a weaker and more poleward ACV barrier compared with NCEP-CFSR. In the reanalyses, the vortex is characterized by narrower and more equatorward bands of high wind-speed and high PV, with higher peak wind-speed and a steeper PV gradient. This leads to a higher maximum $\kappa$ and a more equatorward zone of high $\kappa$, compared with the SOCOLv3 simulations (Figure 5.1d). Peak $\kappa$ at $550 \mathrm{~K}$ is at approximately $63^{\circ} \mathrm{S}$ for both reanalyses, with a FWHM of $11.9^{\circ}$ of $\phi_{e}$. The SOCOLv3 INT, MM3D and MMZM simulations show progressively weaker barriers which maintain similar $\phi_{e}$ peak locations at about $64^{\circ} \mathrm{S}$. It is seen in these figures 
that the two ensemble members for each of the sensitivity runs (i.e., MM3D and MMZM) are almost indistinguishable, indicating that the internal variability of the model does not adversely affect the results from the simulations carried out here, at least at the level of climatological means.
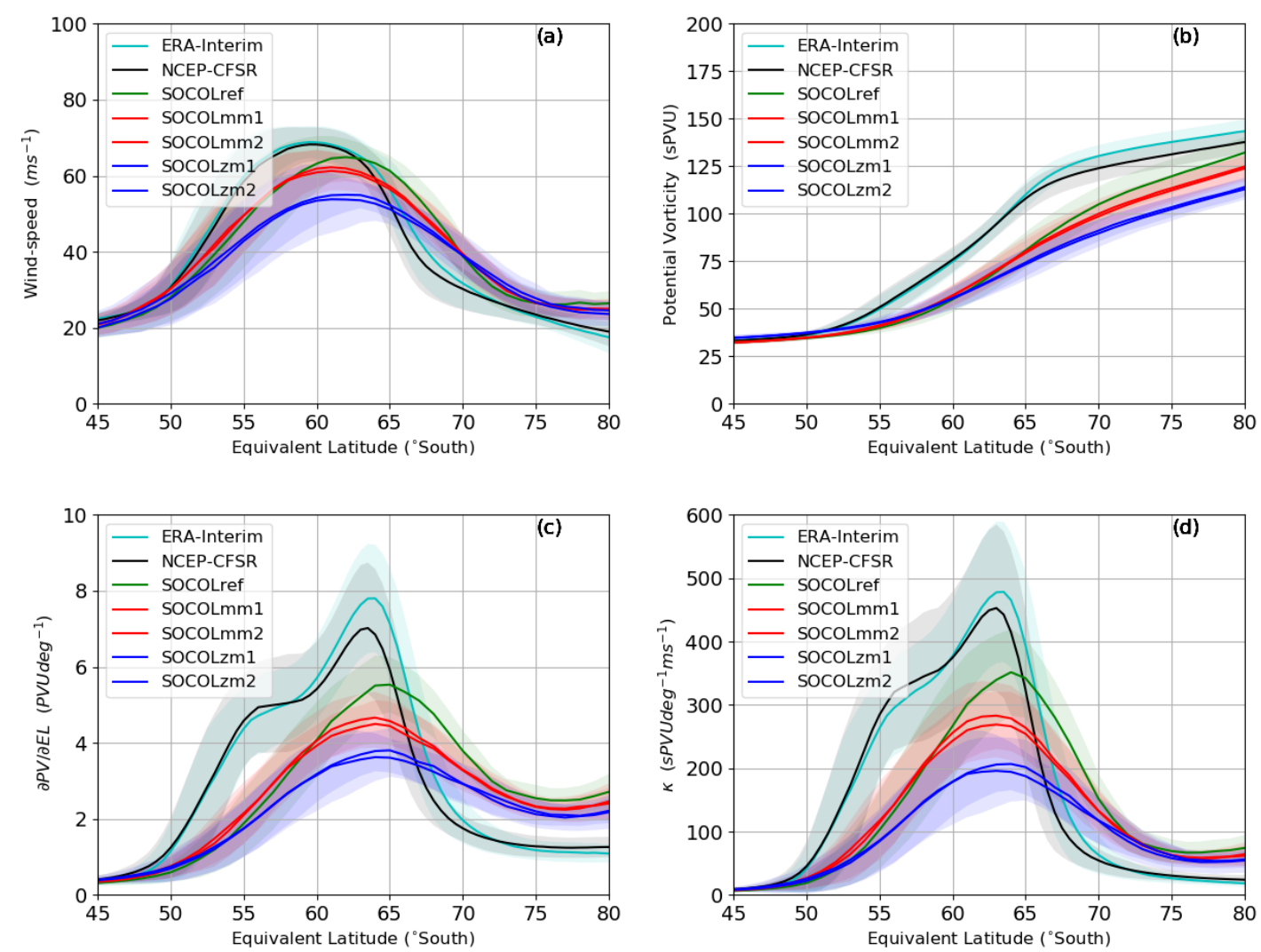

Figure 5.1: September monthly-mean climatology (1981-2009) by equivalent latitude at $550 \mathrm{~K}$ for (a) wind-speed, (b) PV, (c) PV gradient, (d) meridional impermeability $(\kappa)$. Colours identify the SOCOLv3 interactive ozone chemistry simulation (green); the 3-D prescribed ozone simulation (red lines for each ensemble ); the zonal-mean prescribed ozone simulation (blue lines for each ensemble); NCEP-CFSR reanalysis (black); ERA-Interim reanalysis (cyan). The shaded areas denote the spread of values at $1 \sigma$ either side of the mean.

While overlap of the shaded significance levels in the plots provide the first indication of where the curves in Figure 5.1 may be significantly different from one another, a standard t-test was also carried out for the wind-speed and 


\begin{tabular}{|l|r|r|}
\hline Measure & MM3D & MMZM \\
\hline Wind-speed maximum & $95 \%$ & $84 \%$ \\
Wind-speed FWHM & $111 \%$ & $126 \%$ \\
$\kappa$ maximum & $79 \%$ & $57 \%$ \\
$\kappa$ FWHM & $115 \%$ & $127 \%$ \\
\hline
\end{tabular}

Table 5.1: Comparison of selected curve measures for the SOCOLv3 3-D prescribed ozone simulation (MM3D) and the zonal-mean prescribed ozone simulation (MMZM) from Figure 5.1 as a percentage of the interactive ozone chemistry simulation.

$\kappa$ plots. For both variables, significant differences were only found between the reanalyses and the SOCOL-MMZM simulation.

Table 5.1 presents Gaussian fit metrics (maximum and FWHM values) for the MM3D and MMZM ozone simulations shown in Figure 5.1a (wind-speed) and Figure 5.1c $(\kappa)$ as a percentage of the fully interactive simulations, and indicates diminishing fidelity of the representation of ACV impermeability in the SOCOLv3 simulations in moving from MM3D to MMZM, with weaker maximum wind-speeds, weaker maximum $\kappa$, and flatter wind-speed and $\kappa$ profiles. This effect is particularly apparent for comparisons of the maximum values, where using $3-\mathrm{D}$ prescribed ozone improves representation of the wind-speed maximum and the $\kappa$ maximum by $11 \%$ and $22 \%$ respectively, compared with using zonal-mean ozone.

For comparisons of other months at the $550 \mathrm{~K}$ and $850 \mathrm{~K}$ levels (Appendix C), it is apparent that the SOCOLv3 model results consistently simulate a vortex barrier that is too far poleward. At $550 \mathrm{~K}$ in July the vortex is fairly well simulated by each of the model configurations, but is skewed toward the pole while the reanalyses is much more symmetrical. For August at $550 \mathrm{~K}$ the results are more similar to those seen here for September, where the peak strength of the barrier begins to fall below that of the reanalyses. At the $850 \mathrm{~K}$ level the SOCOLv3 model simulations are in fairly close agreement and quite different from the reanalyses. For July the model shows a stronger, narrower and more poleward barrier based on $\kappa$, while in August the model barrier is of similar strength to that shown in the reanalyses, but almost $10^{\circ}$ of equivalent latitude closer to the pole. In September at this level, the SOCOLv3 simulations are considerably weaker than the reanalyses, while maintaining their poleward position. 


\subsubsection{Monthly versus daily data}

A comparison of monthly and daily $\kappa$ is undertaken for the SOCOL simulations for the same years (1984 and 2008) and months (September) examined in Chapter 4 for the UM. In Figure 5.2 the daily and monthly average equivalent latitude profiles are shown for each model simulation and for NCEPCFSR (see Figure 4.2 for ERA-Interim comparison).

No bifurcated $\phi_{e}$ profiles are detected in the SOCOLv3 simulations, and the two ensemble members for the MMZM simulation show quite considerable differences here, while those in MM3D are much closer. This is likely to result from a combination of the inability of the model configurations to simulate the same daily and monthly values as seen in reanalyses, as well as the use of coarser ozone fields. The patterns seen for the NCEP-CFSR and ERAInterim reanalyses are shown in Figure 4.2, and discussed in Chapter 4.

\subsubsection{Time-series analysis}

\section{Annual}

As noted in Chapter 4, the interpretation of trends in reanalysis should be treated with caution, even if they are found to be statistically significant (refer to section 4.2.3). As for the work undertaken for the UM, trends in the annual time series for September mean maximums were evaluated here using simple linear regression, with significance determined on the basis of $\mathrm{p}$ values $(\leq 0.05)$ and a trend was apparent if the fit co-efficient was significantly different from zero at \pm 1 standard deviation $(1 \sigma)$. Note that SOCOLv3 model set-up meant that the years over which the data was assessed (and hence the results reported here for trends in the reanalyses) differs between the UM and SOCOL models.

The time series of the September mean maximum values for wind-speed, $\mathrm{PV}$ and $\kappa$ is shown in Figure 5.3 for the $550 \mathrm{~K}$ level. Over the SOCOLv3 data period, a significant trend in maximum monthly wind-speed (Figure 5.3a) is detected in NCEP-CFSR (increasing at $0.19 \pm 0.07 \mathrm{~ms}^{-1}$ per year over the period, with an R-squared of 0.37 ), while ERA-Interim also increased $\left(0.15 \pm 0.07 \mathrm{~ms}^{-1}\right.$ per year, with an R-squared of 0.24$)$. No significant maximum wind-speed trends were apparent for any of the SOCOLv3 simulations. Wind-speed in the zonal mean (SOCOLzm1) simulations are significantly lower (based on a t-test) than the reanalyses and the other model simulations.

For the September means of the meridional maxima in absolute PV at $550 \mathrm{~K}$ (Figure 5.3b), the lowest absolute PV values are seen in the SOCOLv3 
1984
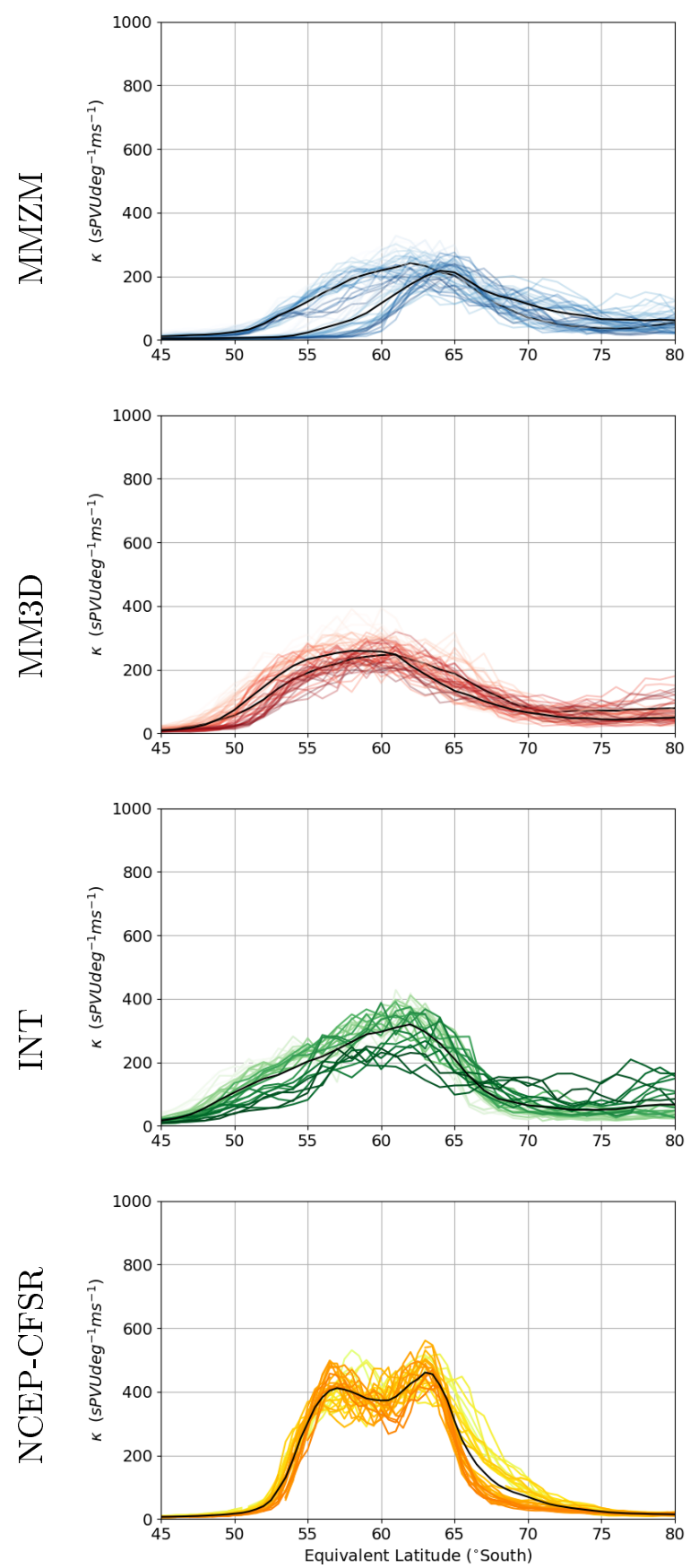

2008
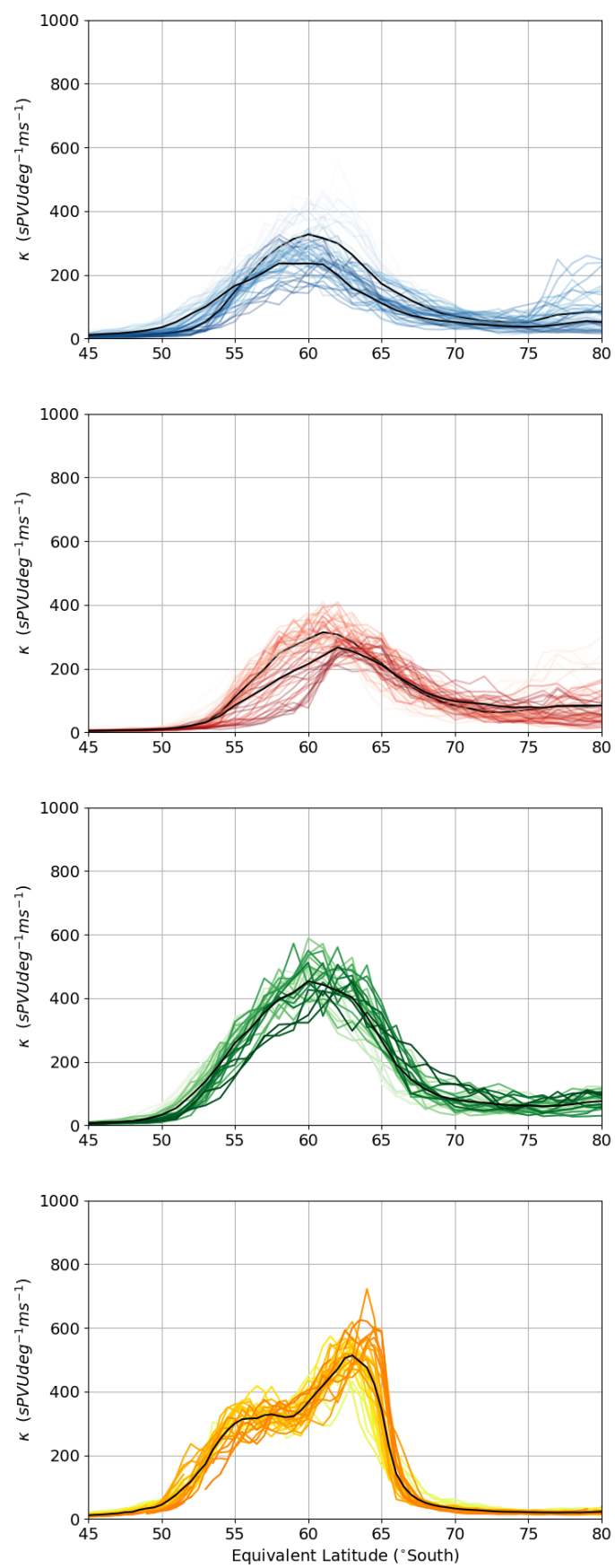

Figure 5.2: Daily (colours) and monthly (black lines) average $\kappa$ profiles by equivalent latitude for SOCOLv3 zonal-mean prescribed ozone simulation (top), 3-D prescribed ozone simulation (2nd row); interactive ozone chemistry simulation (3rd row), and; NCEP-CFSR (bottom) at $550 \mathrm{~K}$. Plots are for September 1984 (left column) and 2008 (right column). Lighter colours indicate the start of the month and darker colours the end of the month. Note that each of the ensemble simulations are shown here for SOCOL-MM3D and SOCOL-MMZM. 

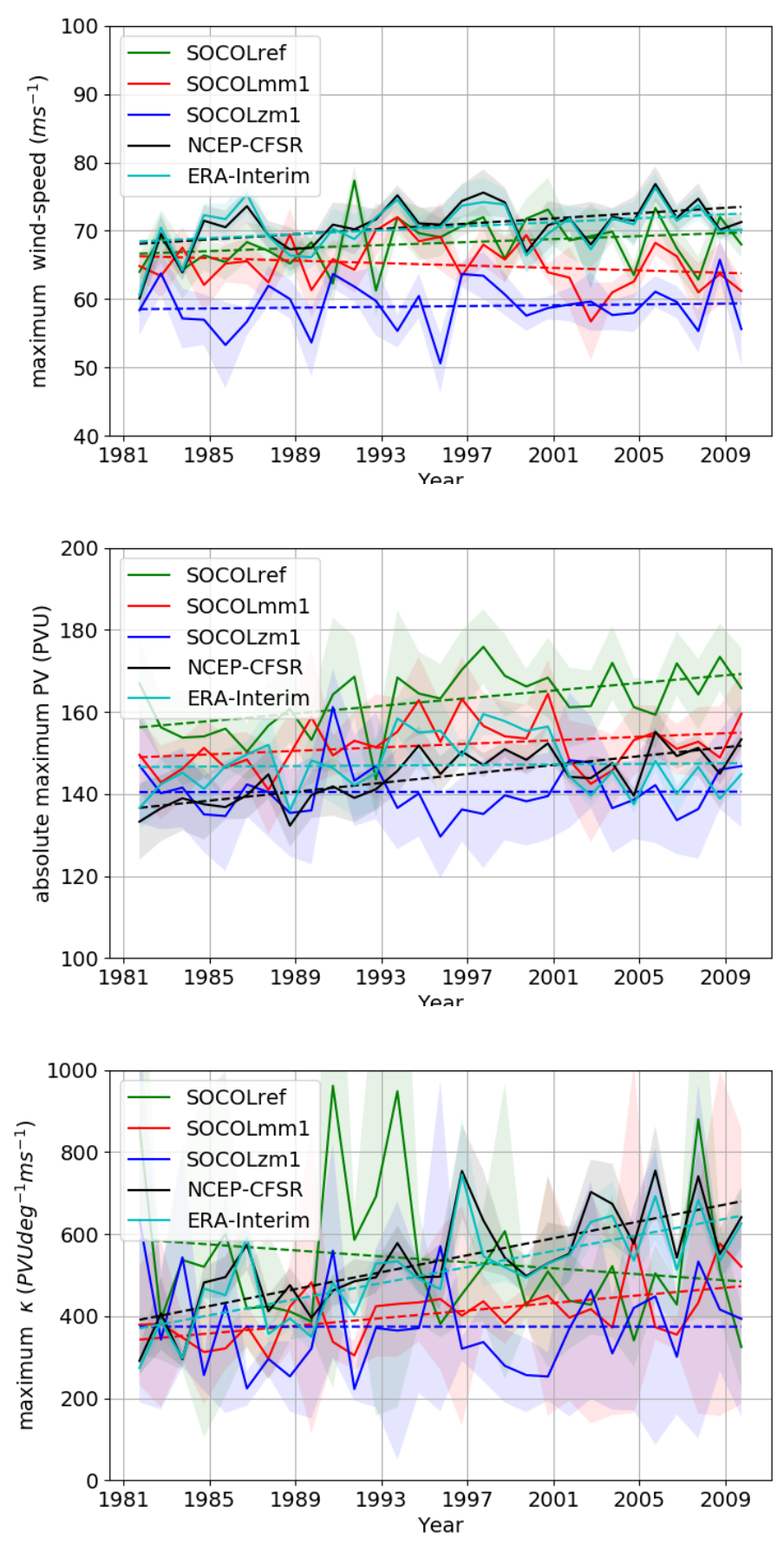

Figure 5.3: Time series of September average (a) meridional maximum windspeed, (b) meridional absolute maximum PV and (c) meridional maximum $\kappa$ values for SOCOLv3-INT (green), SOCOLv3-3D (red), SOCOLv3-2D (blue), NCEP-CFSR (black), and ERA-Interim (cyan) at the $550 \mathrm{~K}$ level (19812009). The maximum monthly average values are chosen from monthly average values calculated for $1^{\circ}$ equivalent latitude steps from $45-85^{\circ} \mathrm{S}$. The shaded areas denote the spread of values at $1 \sigma$ either side of the mean. 
zonal mean simulation (SOCOLzm1), while the SOCOLv3 interactive simulation (SOCOLref) shows the highest PV values. PV increases over the period in NCEP-CFSR (0.54 $\pm 0.09 P V U$ per year, with an R-squared of 0.48$)$, but no trend is apparent in ERA-Interim. The SOCOLv3 reference configuration also shows a positive trend in $\mathrm{PV}(0.46 \pm 0.14 P V U$ per year, with an R-squared of 0.22). No trend in maxima of absolute PV in September is apparent in either of the other SOCOLv3 simulations.

September mean meridional maximum $\kappa$ at $550 \mathrm{~K}$ (Figure $5.3 \mathrm{c}$ ) shows higher values in reanalyses than each of the SOCOLv3 model simulations, with the exception of SOCOLref which is highly variable over the period. Significant positive trends are again apparent in the reanalyses for both NCEPCFSR (10.3 $\pm 1.8 \kappa \mathrm{U}$ per year over the period, with an R-squared of 0.44$)$, and for ERA-Interim $(8.7 \pm 1.6 \kappa \mathrm{U}$ per year over the period, with an R-squared of $0.36)$. A significant trend in maximum $\kappa$ is also found for the $3-\mathrm{D}$ monthly mean simulations (averages across the two simulations of $5.2 \pm 2.0 \kappa \mathrm{U}$ per year over the period, with an R-squared of 0.26 ). No trend in maximum monthly mean $\kappa$ was apparent in either the interactive or the zonal mean model simulations.

\section{Pentads}

Long-term changes in the shape of the $\kappa$ distribution averaged across pentads are shown in Figure 5.4. Each pentad is referred to here by the middle year of the five-year period. As seen in Chapter 4, the maximum $\kappa$ in both reanalyses monotonically increases from 1983 until 2003 and decreases thereafter, consistent with a stronger vortex associated with ozone depletion, as found in previous work (Bodeker et al. 2002; Struthers et al. 2009).

For the SOCOLv3 model, an earlier and lower peak level of $\kappa$ is seen in INT (in 1993), while both MM3D and MMZM pentads show less variability, with no obvious pattern in the maximum $\kappa$ values. However, as seen above (based on the annual data), significant trends in $\kappa$ are apparent for all but the MMZM simulation. Figure 5.4b is almost comparable for 1998 (aqua colour line) with Figure 3e from Struthers et al. (2009) shown here at Figure 5.5 - but while they use the five year average period centered on 1997 for October, here the pentad is centered on 1998 and September. They also use an earlier model version (SOCOLv2 at a horizontal resolution of $3.75^{\circ}$ latitude $\times 3.75^{\circ}$ longitude). The updated INT model used here for SOCOLv3 appears to show only a bias on the poleward side of the $\kappa$ distribution, while their earlier model indicates biases on both sides of the distribution. Overall, it is difficult to make definitive conclusions from this pentad analysis. 

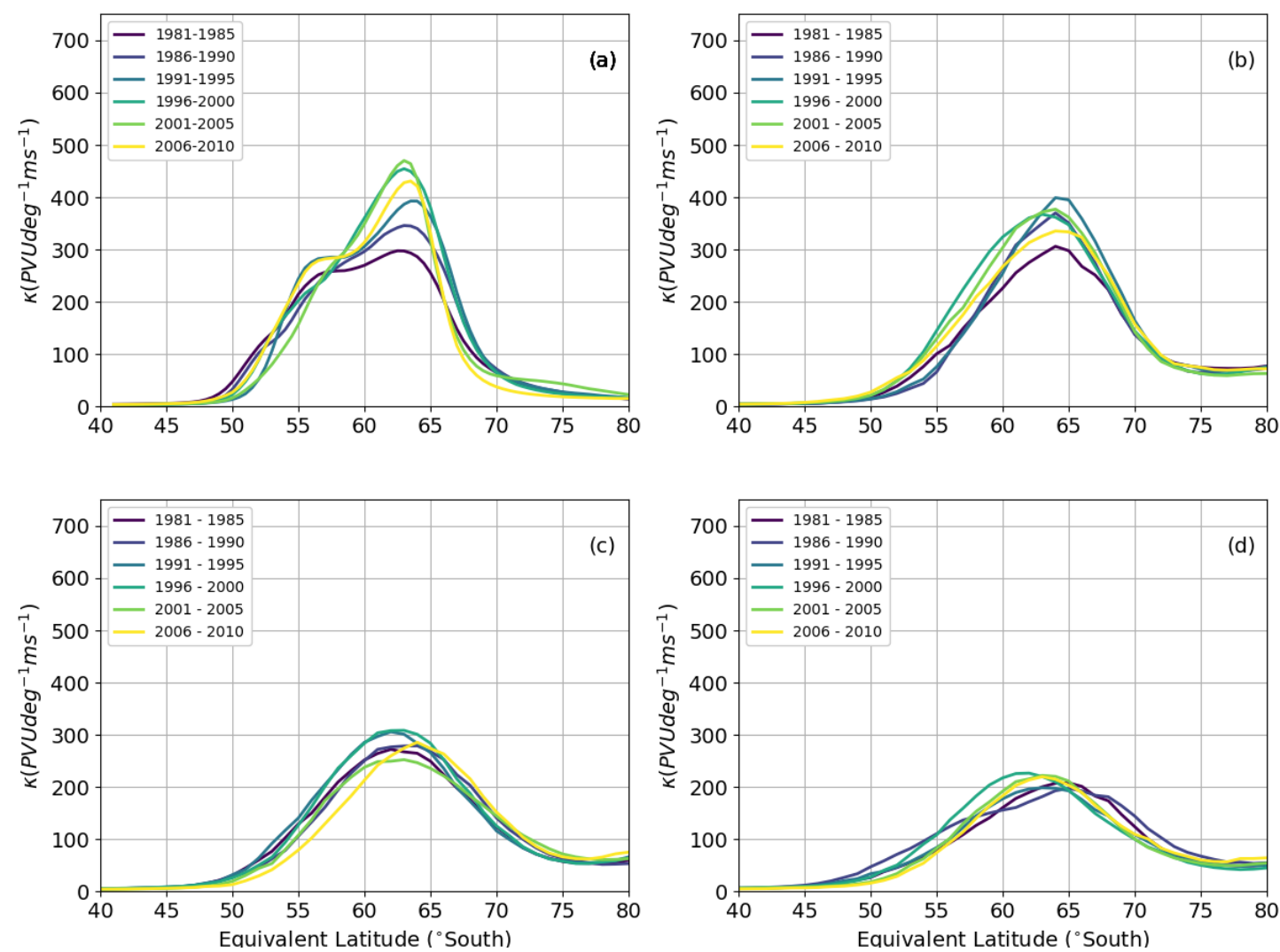

Figure 5.4: Pentads profiles of meridional impermeability $(\kappa)$ by equivalent latitude at $550 \mathrm{~K}$, averaged for September for (a) NCEP-CFSR; (b) SOCOLv3-INT interactive ozone chemistry simulation; (c) SOCOLv3 3-D prescribed ozone simulation; (d) SOCOLv3 zonal-mean prescribed ozone simulation. Note that only the first (unperturbed) simulations are shown here for SOCOL-MM3D and SOCOL-MMZM.

\subsubsection{Vertical profiles}

Cross-sections of $\kappa$ as a function of equivalent latitude and potential temperature are shown for July, August and September, in Figure 5.6. August sees the highest values of $\kappa$ found for any of the three selected months for SOCOLv3, but these do not feature the equatorward lean seen in reanalyses and demonstrate a generally lower, weaker and narrower barrier (i.e., a smaller span of equivalent latitudes with elevated $\kappa$ ). This contrasts with the pattern seen in Figure 5.1 for September at $550 \mathrm{~K}$, where the vortex barrier was seen to be narrower in the reanalyses - however, it is difficult to determine 


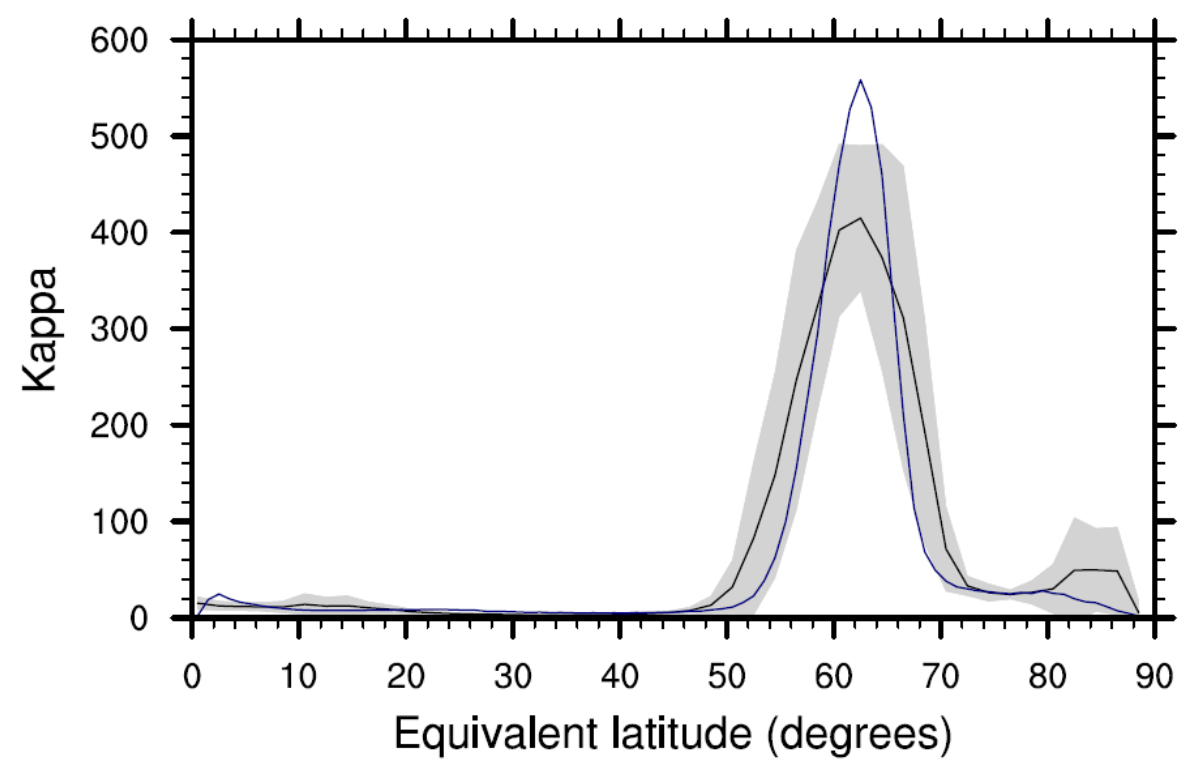

Figure 5.5: Five year (centered on 1997) average October $\kappa$ from SOCOLv2 (black line, where shading indicates the $1 \sigma$ standard deviation about the mean) and NCEP-NCAR reanalysis (blue line). Source: Figure 3e in Struthers et al. (2009).

FWHM values from Figure 5.6.

SOCOLv3 model results for all simulations do not maintain a strong vortex with height in September, and do not indicate a bifurcated $\kappa$ profile as is commonly seen in reanalysis and some model simulations (Conway et al. 2018; Cameron et al. 2019). A degree of bifurcation can be seen in NCEPCFSR here for September below $700 \mathrm{~K}$ (Figure 5.6c), with peaks at around $58^{\circ} \mathrm{S}$ and $62^{\circ} \mathrm{S}$ equivalent latitude.

Even when using interactive ozone chemistry (Figure 5.6d-f), SOCOLv3 underestimates the strength of the ACV, and does not capture the correct shape or meridional extent of the ACV compared to reanalyses. This is consistent with earlier findings, where CCMs with interactive chemistry exhibit varying performance in their simulation of the ACV (e.g., Butchart et al. 2011), which tends to be more poorly represented than its counterpart in the $\mathrm{NH}$. In a range of CCMs, the ACV is too strong in the upper stratosphere and does not tilt sufficiently equatorward with increasing height (Butchart et al., 2011). This is consistent with the SOCOLv3 results shown here, where the tilt of the $\kappa$ barrier seen in NCEP-CFSR in August (Figure 5.6b) is not found in the SOCOLv3 simulations. McLandress et al. (2012) find that this lack of tilt in the vortex simulated in climate models indicates a bias in the 
July
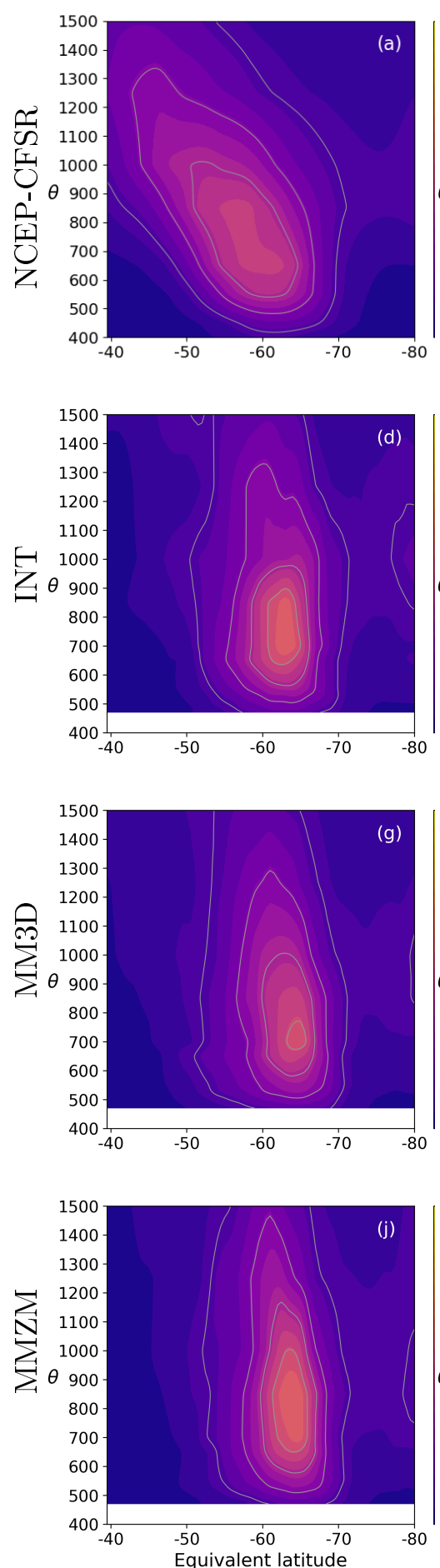

August
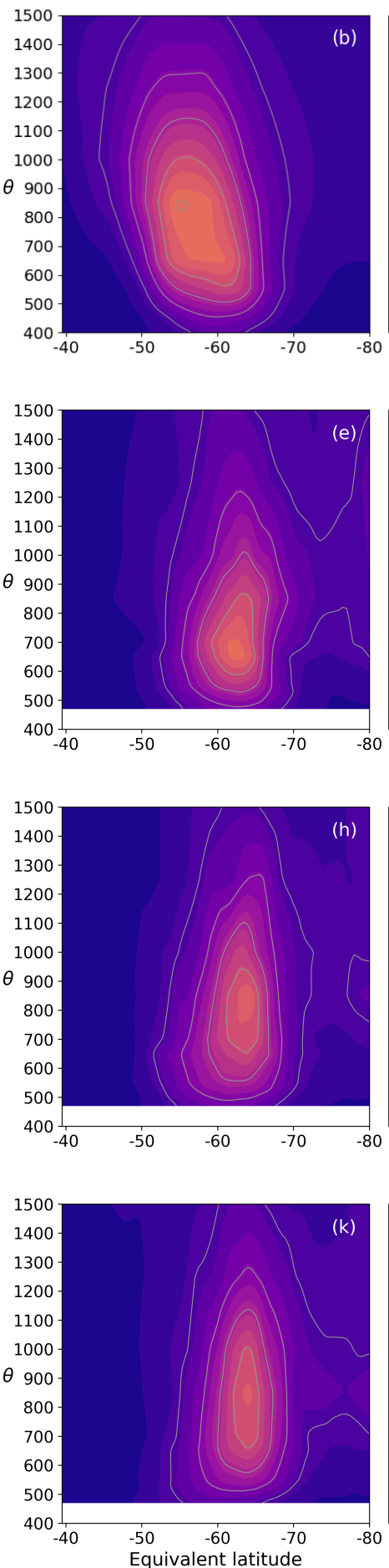

September
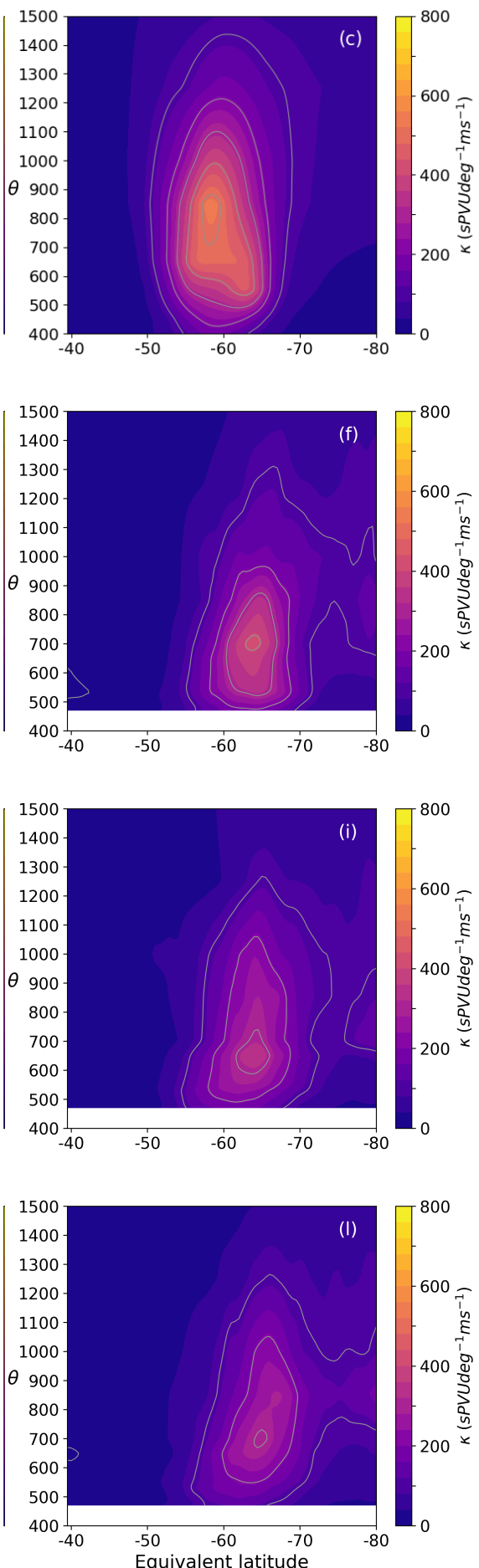

Figure 5.6: Climatological mean (1981-2009) structure of standardised meridional impermeability, $\kappa$ (colours; $\mathrm{s} \kappa \mathrm{U}$, and grey contour lines at 100 $\mathrm{s} \kappa \mathrm{U}$ steps) at levels between $475 \mathrm{~K}$ and $1500 \mathrm{~K}$ for selected months in: NCEPCFSR reanalysis (a)-(c); SOCOLv3 interactive ozone chemistry simulation (d)-(f); the 3-D prescribed ozone simulation (g)-(i); the zonal-mean prescribed ozone simulation (j)-(l). Note that only the first of the ensemble members is shown for SOCOL-MM3D and SOCOL-MMZM. 
model physics attributed to missing components of gravity wave drag.

\subsubsection{Temporal variation in $\kappa$ and ozone}

High variation in ozone concentration is a feature of the SH polar stratosphere, particularly when ozone loss is occurring in the spring period. It has been found that the use of daily ozone in full chemistry model simulations can reduce the model's climate sensitivity, and model results have indicated (e.g., Chiodo and Polvani 2016; Marsh et al. 2016) that while interactive ozone chemistry does not affect the surface temperature response in the $\mathrm{SH}$, it has a considerable effect on stratospheric temperatures. As a starting point to investigate daily, seasonal and interannual variation in both $\kappa$ and ozone, a range of variables are plotted in Figure 5.7 as follows:

- Daily $\kappa$ profile by $\phi_{e}$ (colours: low values in white, moderate values in pink, and high values in green - from NCEP-CFSR reanalysis).

- Maximum monthly value of $\kappa$ (cyan line).

- The daily $\kappa$ FWHM value for the the vortex period (blue dots).

- The daily equatorward and poleward $\phi_{e}$ locations of the half-maximum value of $\kappa$ shown for the vortex period (orange dots).

- The maximum annual extent of the ozone hole converted to latitude coordinates shown on the date of its occurrence (red dots - from NASA: ozonewatch.gsfc.nasa.gov).

- Monthly standard deviation in the first derivative of daily $\kappa$ (blue line), as an indicator of day to day variability within each month.

- The number of days between the annual date of maximum $\kappa$ and the annual date of the maximum size of the ozone hole (green line).

- The daily ozone mass deficit (OMD: red line - from Bodeker et al. 2005 - the mass of ozone (in $\mathrm{kg}$ ) required to return ozone levels over Antarctica to 220 Dobson Units (DU).

Near the start of each vortex season the barrier is wide (typically around 20 degrees of equivalent latitude), becoming narrower throughout each season (to around 5 degrees equivalent latitude width near the end of the season). However, many years show a mid-season widening of the barrier of about 5 degrees, which appears to be associated with a strengthening of the vortex core (i.e., the first appearance of values in the 'green' range in the top contour plot of Figure 5.7), and a flattening out (or often a reduction) in the variance of $\kappa$ (based on the monthly standard deviation of the maximum in the $\kappa$ derivative - lower plot in Figure 5.7). 


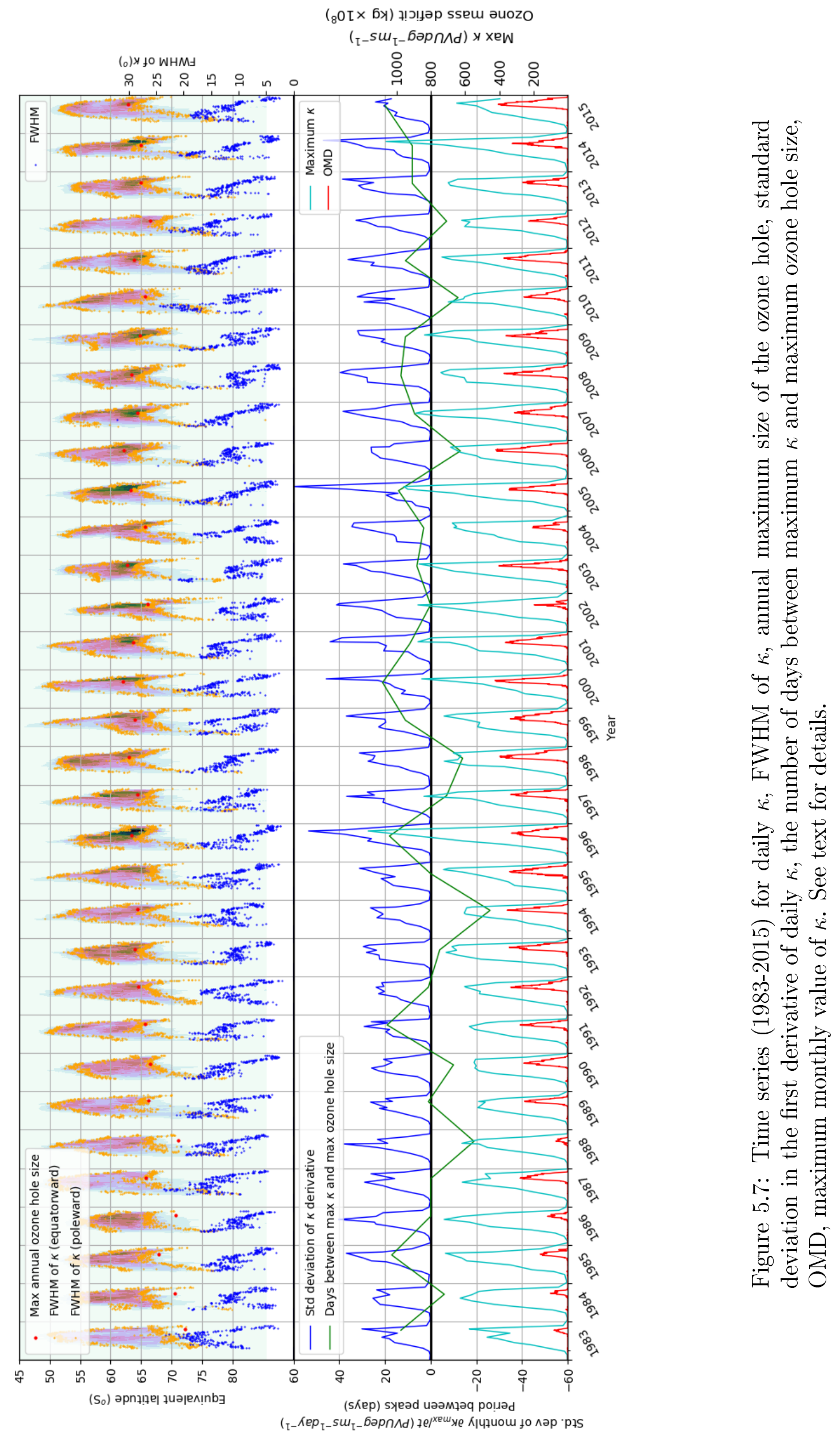


Several years of anomalous vortex seasons are apparent. In particular 1996 stands out, with the strongest barrier by some margin (based on the maximum of $\kappa$ in the reanalyses. Some of the highest rates of change in maximum $\kappa$ (based on the monthly std. deviation of the max $\kappa$ derivative) are also observed in 1996. It is also a year where the ozone hole maximum size had reached its largest extent up until that time, and where the maximum size of the ozone hole occurred almost 20 days before the maximum of $\kappa$.

The 2002 year is unusual due to the unique occurrence of the only recorded major SSW event in the SH, thought to be primarily driven by strong waveforcing in the lower stratosphere (Manney et al., 2005). Both the ozone mass deficit and the ozone hole size are relatively small for that year, with OMD at its lowest since 1988, while the ozone hole size is at its smallest since 1990. Although the maximum $\kappa$ value is reasonably high in 2002 , the vortex width is the narrowest of all years shown here. Interestingly the maximum $\kappa$ occurred on the same day as the maximum in the ozone hole extent (19th September), less than a week before that years SSW event (25th September). Ricaud et al. (2005) show that on 19-20 September 2002 the Antarctic vortex was dynamically stable, but changed dramatically with the change in wind direction and splitting of the vortex at levels above about $600 \mathrm{~K}$ during the SSW event. This led to a shorter than usual vortex season, and less chance of PSC development. The ozone hole during 2004 was also smaller than normal, and the smallest (apart from 2002) since 1991. This coincides with a weaker than normal barrier, with the lowest $\kappa$ maximum since 1994.

Over the period 1983-2015, the size of the ozone hole begins to reach the inner edge of the vortex annulus (based on the poleward FWHM of $\kappa$ ). This is particularly evident in 1996 where the edge of the ozone hole is at about the same latitude as the maximum $\kappa$ value (by equivalent latitude).

The monthly standard deviation of the first derivative of $\kappa$ indicates the degree of fluctuation in the barrier strength. It is closely aligned to the maximum value of $\kappa$ - that is, a year with high maximum values is also likely to experience high variance during that season, as it can rapidly shift between extreme maximum values and lower values.

\section{Regression model}

To establish if there is a link between the extent of ozone depletion and the degree of vortex isolation based on $\kappa$, a regression model was constructed. The model was developed to understand the relationship between daily mean values of $\kappa$ (between $50-70^{\circ} \mathrm{S}$ equivalent latitude) and daily OMD for the Antarctic region (OMD values were calculated as the average over the latitudinal range $40-90^{\circ} \mathrm{S}$ sourced from Bodeker et al. (2005), and smoothed using 
a 3-day running mean).

First, the regular seasonal cycle across all years was calculated using Fourier analysis (with 6 Fourier pairs) and subtracted from each time-series (i.e., $\kappa$ and OMD), leaving the anomalies for each day (i.e., $\kappa$ ' and OMD', where primes denote anomalies). Then, a linear least-squares regression model was developed with OMD as a predictor (i.e., an explanatory variable or basis function), to assess the relationship between mean annual $\kappa$ ' and mean annual OMD'. This included 2 Fourier pairs to allow for dependence of $\kappa$ ' on OMD'. Results showed a $47 \%$ R-squared value (with the linear fit having a slope of $0.11 \pm 0.08$ ) - indicating a significant positive correlation between the strength of the vortex and the ozone mass deficit on an annual basis. A scatter-plot of this relationship is shown in Figure 5.8, together with the phase of the QBO.

\section{QBO phase}

It was speculated that the variation in the annual strength of this relationship may be related to the QBO phase. Monthly mean data was sourced from the long-term Singapore data set commencing in 1976 (available from the Freie Universität, Berlin $\left.{ }^{1}\right)$. Wind-speed on the $70 \mathrm{hPa}$ pressure level was used to determine the phase of the $\mathrm{QBO}$ during the $\mathrm{SH}$ winter and spring months (i.e., 1 June-30 November inclusive), with the QBO characterised as:

- Westerly: winds are from the west for the entire period

- Easterly: winds are from the east for the entire period

- West to east: winds switch from west to east during the period

- East to west: winds switch from east to west during the period

While there is no obvious pattern apparent for the QBO phase, it appears that most of the 'east-to-west' and 'west-to-east' phase years occur with higher $\kappa$ anomalies. Years with an easterly phase of the QBO mostly correspond to high OMD anomalies. Further analysis would be needed to assess the nature of these possible relationships in more detail.

\section{EESC}

Finally, the annual mean anomalies ( $\kappa$ ' and OMD') were compared over time against annual effective equivalent stratospheric chlorine (EESC), an indicator of the total volume of ODSs in the stratosphere. EESC data (in ppt) were sourced from Newman et al. (2007) using the WMO A1 scenario,

\footnotetext{
${ }^{1}$ www.geo.fu-berlin.de/met/ag/strat/produkte/qbo/qbo.dat
} 


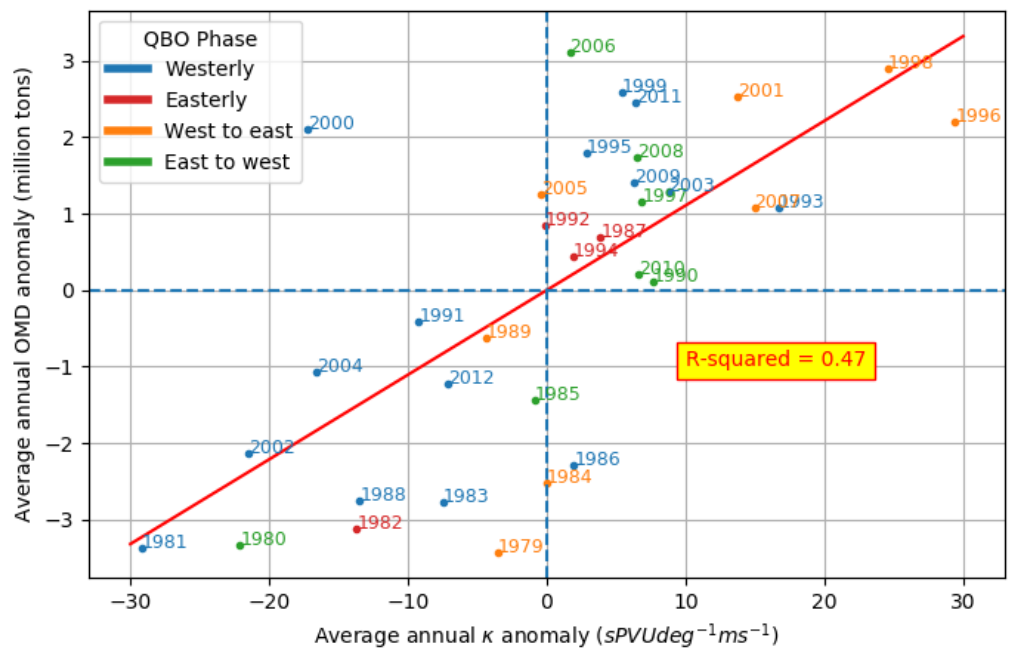

Figure 5.8: Scatter-plot of annual anomalies in $\kappa$ and OMD for the years 1979-2014 from NCEP-CFSR, with the red line indicating the straight-line regression fit, and the colours for each year showing the QBO phase at $70 \mathrm{hPa}$.

with a mean age of air of 7 years, an age of air spectrum width of 3.5 years, and a bromine scaling factor of 60 . The results of this analysis are shown in Figure 5.9, where it can be seen that both $\kappa$ ' and OMD', correspond closely with the pattern of increasing, followed by decreasing EESC values. 


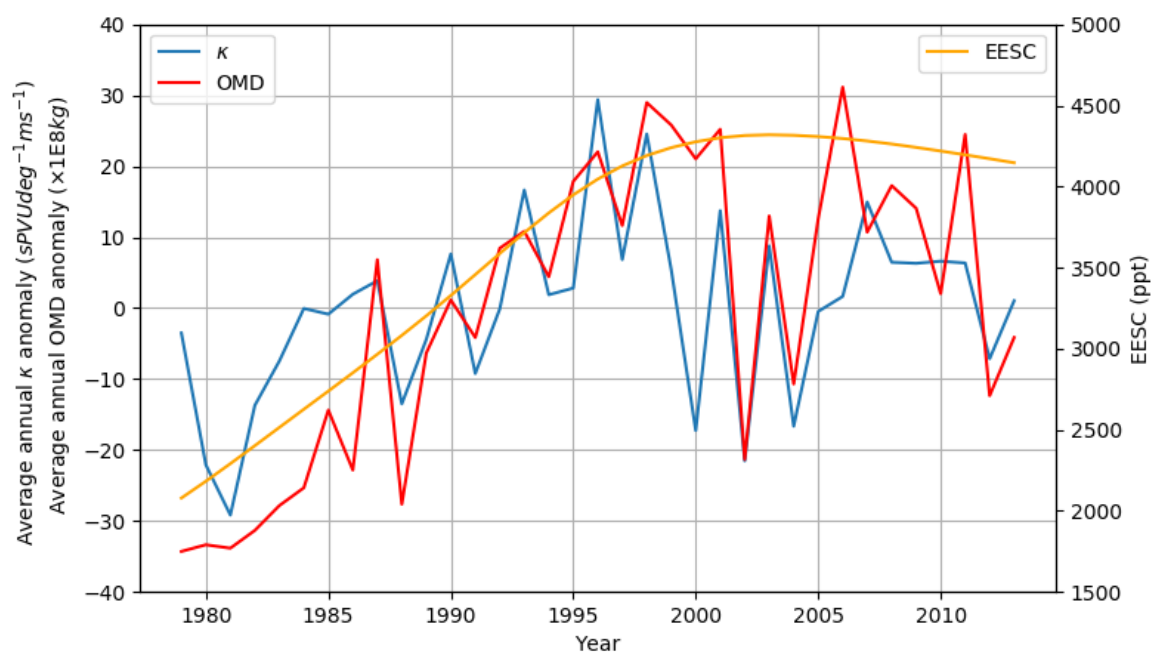

Figure 5.9: Time series of the annual average anomaly in ozone mass deficit (OMD), the annual average anomaly in $\kappa$, and the annual equivalent effective stratospheric chlorine (EESC). Data sources are: $\kappa=$ NCEP-CFSR reanalysis; $\mathrm{OMD}=$ Bodeker et al. (2005); EESC=Newman et al. $(2007)$

\subsection{Discussion and conclusions}

It is shown here that for the $550 \mathrm{~K}$ level in September the strength of the $\mathrm{ACV}$ diminishes as the number of dimensions of the prescribed ozone field reduces - from the strongest $\kappa$ values when using interactive ozone, reduced $\kappa$ using 3-D monthly averaged ozone climatology, and the lowest $\kappa$ values when simulations use monthly-mean zonal-mean (i.e., 2-dimensional) prescribed ozone. Although the use of interactive ozone chemistry in a CCM provides the greatest fidelity of the representation of the ACV, it remains computationally expensive. The use of time-varying 3-D monthly ozone fields in climate models is recommended in preference to time-varying 2-D monthlymean zonal-mean ozone fields.

Prescribing monthly-mean zonal-mean ozone in the stratosphere of a GCM can result in significant spatial and temporal mismatches between the internal model dynamics and the radiative forcing field from ozone (Crook et al. 2008; Neely et al. 2014). These mismatches lead to erosion of the steep gradients in temperature, potential vorticity and trace gases across the vortex edge and consequent flattening of meridional $\kappa$ profiles. The results displayed here for SOCOLv3-MMZM are consistent with such mismatching.

It is likely that the weaker vortex that is simulated when prescribed ozone 
fields are used also weakens the feedback mechanism that causes the vortex to become self-reinforcing (see section 2.2.4 above), thus acting to further reduce the ability of the model to accurately represent the form of the vortex. One potential solution for this may be to include a component that represents this self-reinforcing feedback mechanism as a prescribed factor in climate models.

One important point to note with the SOCOLv3 model simulations is that the prescribed ozone fields are consistent with the model dynamics that is, they are derived from the earlier interactive simulation. This consistency reduces the likelihood that the fields will mismatch, and therefore the prescribed ozone model simulations are closer to the interactive reference simulation than would be the case in a simulation when an alternative externally sourced ozone field is used. For example, the model simulations carried out for the UM in Chapter 4 used an ozone data set that was created for the Coupled Model Intercomparison Project (CMIP) phase 6 (Checa-Garcia et al., 2018), so did not have internal consistency with the UM dynamics, thus potentially contributing to the differences seen in those model results. Some previous researchers (e.g., Crook et al. 2008) have also used GCMs with ozone fields from external sources, which are also likely to have differences that were exacerbated due to the inherent discrepancies between the model dynamical fields and the ozone field. However, other experiments (e.g., Waugh et al. 2009, Gillett et al. 2009; Neely et al. 2014) used ozone fields that were sourced from the model itself - i.e., using CCMs - thus reducing the likelihood of inconsistencies from that source.

In the 3-D model, inclusion of the longitudinal dimension provides an additional level of variation, in comparison to the $2 \mathrm{D}$ simulation, that is likely to be ensuring closer correspondence of ozone and dynamical fields, and be the main factor in the improved ACV representation. Further experiments using simulations such as those undertaken here would be useful to further test the correspondence of the mean state and the influence of waves. For example, assessment of the level of correlation between the 2-D and 3-D ozone fields and the GPH fields at say $20 \mathrm{hPa}$ (indicating zones of higher or lower pressure).

These results suggest that use of 3-D monthly-mean ozone climatology in climate models is preferable to the use of monthly-mean zonal-means, where improvements are seen in the representation of the maximum $\kappa$ value by $22 \%$ in the climatology at $550 \mathrm{~K}$ (see Table 5.1) - with a wider range of improvement expected for individual years. Such results are likely to be applicable to other climate models, although this would require further verification.

While SOCOLv3 is not able to fully capture the meridional shape and 
strength of the ACV in the fully interactive simulation, this is commonly seen across a range of CCMs. Struthers et al. (2009) found that the $\kappa$ distribution in CCMs is highly related to the model dynamics, and less related to the spatial distribution of radiatively active gases or the model chemistry scheme. Though SOCOLv3 exhibits a cold temperature bias in the polar lower stratosphere, and an overestimation of polar ozone loss during the Antarctic spring (Stenke et al., 2013), such biases are also seen in other non-spectral models, and are usually related to the advection scheme used.

As computing power improves, climate models would be expected to increasingly simulate interactive ozone chemistry, rather than prescribed ozone fields. Until then, the results presented here suggest that there are some gains to be had in representing stratospheric processes by prescribing a $3-\mathrm{D}$ stratospheric ozone field rather than a zonal-mean stratospheric ozone field.

A regression model that was developed to determine the relationship between $\kappa$ and the severity of Antarctic ozone depletion (Figure 5.9) showed a strong correlation between the strength of the vortex and the ozone mass deficit, and also tracked closely with the equivalent effective stratospheric chlorine (EESC), an indicator of the concentration of ODSs in the stratosphere. 
120 CHAPTER 5. THE VORTEX BARRIER IN THE SOCOL MODEL 


\section{Chapter 6}

\section{Discussion}

Little work has previously been undertaken on many aspects of the research described within this thesis, including comparing mixing metrics in the $\mathrm{SH}$ polar region, evaluating the benefits to be gained in representing the $A C V$ transport barrier by comparing GCM versions, or in comparing the fidelity of the ACV barrier representation based on the dimensionality of ozone fields used. In the introduction to this thesis (Chapter 1) several research questions were developed. In this chapter each of those questions is revisited and assessed as to how effectively they have been addressed.

\section{What is the ACV, and what role does it play in the stratosphere?}

As detailed in Chapter 2, the ACV is the Earth's strongest atmospheric circulation and plays a critical role in the global and SH winter circulation. In short, the ACV consists of an annulus of strong westerly zonal winds that surround the pole in winter each year, and act as a barrier to meridional transport of air parcels in the stratosphere as the meridional temperature gradient reaches its maximum. The peak strength of the vortex (its maximum impermeability) occurs around August each year. The vortex is then eroded by wave-breaking on its equatorward edge as the temperature gradient between the equator and the pole reduces.

In the context of the research undertaken here, vortex detection is based on the absence of mixing across a meridional profile extending from the midlatitudes to the pole. In Chapter 3 numerous metrics that describe mixing and indicate the vortex transport barrier were shown, based on measures such as the PV gradient, wind-speeds, distance of air parcel transport over a set time (or time for travel over a set distance), the length of an air parcel trajectory (or transport along $\mathrm{PV}$ isolines), air parcel transport across PV 
isolines, and the deformation of material surfaces.

Several long-term changes have been observed in the nature of the ACV, where it has become a stronger and more persistent barrier (e.g., Bodeker et al. 2002), which is primarily attributed to ozone depletion (e.g., Thompson et al. 2011). Reduced ozone leads to reduced heating within the vortex core, thus increasing the temperature gradient between the pole and equator. The ACV has also been found to have moved east off the pole since the 1960s (e.g., Hassler et al. 2011), which is also thought to be driven by ozone depletion.

The ACV plays important roles in the global circulation. The strength and position of the ACV is known to link to changes in the surface climate - primarily in influencing the SAM (e.g., Orr et al. 2012; Gerber 2012). However, our understanding of the mechanisms connecting the dynamics of the stratosphere and the troposphere is still lacking (e.g., Gerber 2012; Kidston et al. 2015). The key aspects of the ACV that have been examined here are its role as a barrier to meridional transport, which links closely with its role as a containment vessel for the processes that lead to ozone destruction, and how effectively climate models represent the ACV barrier.

The role of the vortex as a barrier to transport involves it isolating stratospheric air above the Antarctic from that in the mid-latitudes. Although a wide range of metrics exist for describing this isolation, there is no single metric that is considered to be the 'gold standard' for the purposes of defining transport barriers in the stratosphere. A key outcome from Chapter 3 was a critical assessment of four commonly used metrics to reveal what they each indicate about mixing and the dynamical containment of the ACV. The range of metrics examined here are found to have differing strengths and weaknesses, and may be considered suitable for different applications. For example, while $M$ is found to be well suited for defining mixing that occurs equatorward of the vortex, $\kappa$ is seen to be better at defining the nature of the vortex itself, including identifying bifurcation across the vortex annulus (i.e., where some mixing does occur). This bifurcation tendency that is common to some heights and periods of the season is also found to a lesser extent in the EL metric. Although noisy, CC appears to be effective at defining three distinct periods (vortex formation, peak vortex period, and vortex break-up).

The choice of metric to use, of course, may relate to the complexity of calculation, and here again, meridional impermeability appears to be the best substitute for mixing metrics that require complex trajectory analysis. In Chapter 3, comparisons between metrics have shown strong linear, exponential and parabolic relationships together with signs of hysteresis in some relationships. There is scope for taking this initial determination of relationships between mixing metrics much further to investigate the detailed 
physical mechanisms associated with both the form of the relationships and any apparent hysteresis, as well as their level of statistical significance.

The ACV was shown to have an important role in containing the area of the ozone hole and enabling the conditions in the stratosphere that facilitate the processes leading to ozone destruction. SH ozone affects stratospheric chemistry, circulation, and surface climate (e.g., Thompson and Solomon 2002; McLandress et al. 2011; Polvani et al. 2011). In this thesis, the effect of differing approaches to prescribing ozone in CCMs has been investigated (Chapter 5), where a regression model was developed that showed a strong link between ozone depletion and the strength of the ACV barrier, also aligning with the level of EESC in the stratosphere.

\section{What are the processes that determine the dynamical attributes of the ACV?}

While both the transport barrier and its role in ozone loss have been discussed above, several other processes are important in contributing to the dynamical attributes of the ACV. These include the Brewer Dobson Circulation, the QBO, stratospheric aerosols, wave forcing, wave-breaking within the surfzone, and changing greenhouse gas concentrations. Of these processes, the $\mathrm{BDC}, \mathrm{QBO}$ and stratospheric aerosols were not investigated further, however aspects of the surf-zone and the importance of changes in GHGs have been topics of interest in this thesis, and these are both discussed further below.

Planetary waves and gravity waves are both known to play an important role in stratospheric dynamics, including vortex dynamics. In particular, planetary waves propagate upward from the troposphere to the stratosphere, weakening the mean flow, and leading to erosion of the vortex edge and reduction in vortex strength when they break (e.g., McIntyre and Palmer 1984; Juckes and McIntyre 1987). Planetary waves are also the primary driver of the BDC (e.g., Haynes et al. 1991; Holton et al. 1995; Thompson and Solomon 2002). Increasing GHG concentrations are expected to strengthen the BDC (Butchart, 2014), leading to a stronger meridional circulation, which may be expected to weaken the ACV. However, a stronger BDC will also alter rates of transport of trace gases including ozone, which will feed back into vortex dynamics, making it more difficult to predict how the vortex might alter in the future.

Wave breaking is responsible for erosion of the vortex as it weakens toward the end of each season. In this thesis, evidence of increasing waviness in the surf-zone is indicated by increases in the EL metric, which appears to occur in tandem with a stronger vortex. Further work is required to examine how 
this stronger mixing activity in the surf-zone may be linked with the polar vortex (e.g., through more rapid erosion of the vortex edge), and how these patterns may change in the future. The differences seen here in representation of the ACV in metrics between the start and end of the vortex season may be readily explained by the absence/presence of wave activity, and in particular wave-breaking - leading to the hysteresis signals detected in some metrics.

Planetary waves of different wave-number are known to result in changes to the vortex dynamical structure. For example, zonal wave-numbers 1 and 3 have been shown to cause displacement of the vortex away from the pole (e.g, Waugh and Randel 1999; Ialongo et al. 2012; Grytsai et al. 2017), while zonal wave-number 2 is known to distort the vortex away from circularity (e.g., Waugh and Randel 1999). Planetary wave-number 2 activity is also thought to have contributed to the first major SSW event in the SH in 2002 (Peters et al., 2015). Improving our understanding of such connections between planetary waves and the shape, orientation, strength and size of the vortex would be expected to lead to better understanding and forecasting of SSW events in the future.

Anticipated ongoing change in the polar vortices is a key issue for climate modelling, as the atmosphere undergoes both ozone recovery and increases in concentrations of GHGs. Increased GHG concentrations lead to stratospheric cooling, which may enhance ozone destruction and alter vortex dynamics. Current projections indicate that stratospheric cooling (from GHGs) will be offset to some extent by expected warming (from ozone recovery)(e.g., McLandress et al. 2011). However, the net effect on the polar vortices is thought to be dependent on changes in wave activity entering the stratosphere (Waugh and Polvani, 2010) as discussed above. Such changes in wave activity can be driven by both stratospheric and tropospheric climate change.

While no experiments or analysis involving changes in concentrations of GHGs were undertaken here, its importance to future evolution of the vortex means that improved representation of the vortex for historic data - which has been the focus of this thesis - is the first step toward ensuring that model projections are reliable in their representation of the ACV.

\section{What processes relevant to the dynamical attributes of the ACV may be missing or ill-defined in GCMs?}

Correctly representing the ACV transport barrier in climate models is important because the barrier affects the morphology of radiatively active gases and produces a fingerprint on the climate system in the troposphere, includ- 
ing through its influence on the SAM. Previous researchers have identified deficiencies in the ability of climate models to capture the complex dynamical and chemical processes that control and define the ACV (e.g., Butchart et al. 2011; SPARC 2010; Waugh and Polvani 2010; Deser et al. 2012; Struthers et al. 2009; Scaife et al. 2002; Garcia et al. 2017). While it is widely acknowledged that it is important that the polar vortices are well resolved in climate models, it is apparent that this has not yet been achieved. Such concerns are echoed here, where simulations in both a GCM (Chapter 4) and CCM (Chapter 5) have shown an ACV that is not well represented when compared with reanalyses.

The multi-model comparison of SPARC (2010) found that while models can simulate the isolation of the ACV, its shape is unrealistically distorted from circularity, and the models struggle to simulate the orientation and equatorward tilt with height of the vortex barrier. Waugh and Polvani (2010) find that GCMs show little agreement in either wave action or vortex strength, making it unclear how much confidence can be placed in projections of the polar vortices in climate models.

Previous research, together with the work undertaken here, has identified several possible causes for the discrepancies in results between models and reanalyses. Some key processes that are known to be missing or ill-defined are examined further below. Improvements in these areas should result in better simulation of the ACV, and in increased confidence in projections of future vortex behaviour (and in SH climate more generally).

The way in which the ozone field is simulated is an important aspect of climate modelling due to its impact on model dynamical structure, physics and chemistry. Previous work has determined that representation of stratospheric processes are inadequate due in part to the approach taken to including ozone in the models (e.g., Crook et al. 2008; Gabriel et al. 2007; Gillett et al. 2009; Waugh et al. 2009). Albers and Nathan (2012) proposed that two pathways link zonally asymmetric ozone to stratospheric dynamics (including dynamics of the polar vortex). The first is the direct impact on planetary wave propagation through zonally asymmetric heating; the second effect relates to changes in zonal-mean temperature, altering ozone and modulating the thermal wind balance. Here, improved representation of the vortex is found when using prescribed 3-D ozone in SOCOLv3 simulations, compared with using 2-D prescribed ozone (Chapter 5). These results are consistent with mismatching between model dynamics and model ozone fields - when the ozone fields do not capture the nuances of changing ACV shape or the full effects of ozone depletion.

While the model simulation with interactive ozone chemistry provides 
even better representation of the $\mathrm{ACV}$, this approach is computationally expensive. However, computational cost is becoming less of a barrier as computing power continues to increase, and it is expected that an increasing number of models will simulate interactive stratospheric chemistry over time. In the intervening period, it is recommended that models prescribe longitudinally resolved (i.e., 3-D) ozone, as it is no more computationally demanding than prescribing a zonal average $(2-\mathrm{D})$, but considerably improves the representation of the ACV. Adoption of this recommendation should improve the ability of the climate models used (e.g., by the CMIP series of experiments under the WCRP), to simulate expected future changes in the climate of the southern mid-latitudes.

Even with interactive chemistry, representation of the polar vortex by CCMs is still lacking, and does not correspond with reanalysis - typically the ACV remains located too far poleward and its maximum strength is underestimated. These discrepancies may be primarily due to the model dynamics (e.g., Struthers et al. 2009) - including the effect of GWD parameterizations (see below). However, it is likely that other factors also contribute, such as model resolution and the challenges that models face in incorporating feedback mechanisms.

The numerical scheme used in model cores has been examined here by comparing representation of the ACV between UM model versions (based on different generations of Global Atmosphere and dynamical cores - Chapter 4). Although improvements are seen in moving to a more recent generation of the UM dynamical core, it is also apparent that some aspects of the ACV can be more poorly represented. Specifically, ACV representation is better in the more recent model configuration (GA7.0) in the lower and middle stratosphere (up to around $600 \mathrm{~K}$ ), but performs better in the older UM configuration (GA3.0) above that level. Although the detailed cause of this is not clear due to multiple changes between different model generations, it is likely that an historic focus on tuning climate models for tropospheric applications (i.e., their ability to accurately represent surface climate) has naturally led to less focus on stratospheric representation. However, increased efforts are underway to test and compare models and reanalyses for their ability to simulate stratospheric processes (e.g., SPARC 2010; Fujiwara et al. 2017).

Unfortunately, restriction on computing resources precluded additional sensitivity simulations in the UM, which would have allowed for exploration of the possible causes of discrepancies between the model results and the reanalyses. For example, comparing simulations where selected components were variously activated or deactivated, or by altering the model physics for selected components. It is recognised that such simulations would likely have 
contributed significantly to the results assessed here - and such approaches are recommended for future work.

Missing or ill-prescribed GWD is frequently identified as a key factor in model misrepresentation of the polar vortices (e.g., Waugh and Polvani 2010), and improvements in representation of the ACV may be expected by fine-tuning model parametrization of GWD. In particular, an additional component to increase the deceleration of upper air westerlies may be required in models to enhance planetary waves. This 'missing' forcing which is not currently resolved in climate models, may be due to excessive removal of eastward propagating gravity waves (Manzini et al., 2003).

Results from both the UM and SOCOLv3 model simulations demonstrated a poleward displacement of the inner vortex edge, consistent with previous work (e.g., Morgenstern et al. 2009; Hardiman et al. 2017) and attributed to NOGWD. Orographic gravity waves (OGWs) have also been found to affect the strength and tilt with height of the vortex (e.g., Scaife et al. 2002; Garcia et al. 2017), and inaccuracies in their parameterization are thought to be responsible for generally poor model performance in the stratosphere (McLandress et al., 2012).

While it is speculated that the results showing high impermeability at upper levels in UM-GA7.0 (ENDGame) may result from reduced damping of GWD within the ENDGame dynamical core - thus leading to higher windspeeds and a stronger vortex barrier in the upper stratosphere - further research would be required to validate this.

Improving the parameterization of GWD in GCMs is likely to result in considerable improvement in how well models represent key processes that control the morphology of the ACV. It is recommended that climate modellers, particularly those involved in the development of the NZESM, test the effect of altering the parameterization of GWD, and the impact seen on the ACV and stratospheric processes more generally. It should be noted that the cold-pole problem is also attributed to inaccuracies in wave drag parameterizations used in models (McLandress et al., 2012), and model tuning for GWD should seek to address both improved vortex representation, and the cold-pole problem.

The resolution of model simulations and thus their ability to capture the dynamic processes related to the ACV is demonstrated by Waugh and Polvani (2010), where they note that wave propagation and breaking are sensitive to the PV gradients at the edge of the vortex, with enhanced vertical propagation and breaking associated with steeper-edged gradients. Greater resolution can thus detect finer degrees of variation in these gradients, and more accurately model wave activity. 
Model resolution is a factor that evolves in parallel with increased computing power, and finer resolutions, particularly in regional scale models, are increasingly being used. Together with some of the other factors identified here, higher resolution global models will be needed to achieve improved results in ACV representation. It is expected that increasing the resolution of stratospheric dynamics in models will lead to greater levels of confidence in their simulations, including for projections. While model resolution was not tested explicitly here, it is noted that the model simulations undertaken were on a coarser resolution $\left(1.25^{\circ}\right.$ latitude $\times 1.875^{\circ}$ longitude in the UM and $2.8^{\circ}$ latitude $\times 2.8^{\circ}$ longitude in SOCOLv3 $)$ than the reanalyses $\left(0.5^{\circ} \times\right.$ $0.5^{\circ}$ in NCEP-CFSR and $0.75^{\circ} \times 0.75^{\circ}$ in ERA-Interim), which is expected to have influenced the results examined here, though the specific effects of these differences have not been analysed.

Several positive feedback mechanisms that act to strengthen the ACV have been discussed, including vortex isolation leading to an enhanced temperature gradient, and thus a stronger vortex. While no specific experiments were carried out here to investigate the role of positive feedbacks, it appears that little research activity has been undertaken on this aspect of climate modelling in the polar regions. A major positive feedback mechanism in models already discussed is insufficient planetary wave driving, causing a too strong vortex as the barrier is not eroded sufficiently. In climate models, feedback processes can also depend on the model resolution (e.g., Bony et al. 2006) as discussed above.

Although the ACV has been found to now have a longer season (Haigh and Roscoe, 2009), climate models overestimate this persistence. Such overestimation has also been attributed to positive feedback mechanisms (e.g., Thompson and Wallace 2000; Eyring et al. 2006) and there may be potential in directly including positive feedback mechanisms in model structure. 


\section{Chapter 7}

\section{Conclusions}

This chapter provides a summary of the work carried out in this thesis and recommends future research.

Improved understanding of stratospheric mixing, including defining the lowmixing vortex barrier, and representation of the ACV in GCMs, are of interest to a wide audience in the atmospheric science community. Processes associated with the ACV are of particular importance due to their role in ozone depletion, and the impact that this depletion has on coupling between the stratosphere and the surface climate.

This thesis has provided an improved understanding of the morphology and impermeability of the ACV transport barrier, and has identified several key elements of particular interest, including exploration of mixing diagnostics in the ACV region; identified that current climate models tend not to simulate the ACV barrier accurately, and; indicated that there are benefits to be gained in ACV simulation by using $3-\mathrm{D}$ prescribed ozone in models in place of 2-D ozone.

A range of metrics were evaluated using two modern-era reanalyses to gain insight into their ability to simulate the ACV barrier and associated mixing. Results indicated that meridional impermeability is an appropriate metric for determining the detailed cross-vortex properties of the ACV.

Using an AOGCM - namely the UM in two configurations - simulation of the impermeability of the ACV was investigated based on comparisons of meridional impermeability with reanalyses. The newer model version provided an improved representation of the vortex compared with the earlier model version at lower levels in the stratosphere - while the earlier model showed better representation at higher levels. Both UM versions show discrepancies from the reanalyses associated with the strength and meridional 
width and position of the ACV.

Using a chemistry climate model - namely the SOCOLv3 CCM - provides improved representation of the ACV transport barrier compared to the UM results. The ACV was most closely represented using model simulations with interactive chemistry, while simulations which used either $3-\mathrm{D}$ or $2-\mathrm{D}$ prescribed ozone performed progressively worse. It is recommended that in the absence of interactive chemistry, a 3-D ozone field should preferably be prescribed in climate models.

Projections of climate change require accurate simulation of the past climate. Where processes are not well represented in climate models, demonstrated improvements in modelling of historic climate are first needed to increase reliability in their projections. The NZESM provides the basis for projections of future changes in New Zealand's climate, and NZESM simulations drive impact models which are used to quantify and assess the impacts of climate change on New Zealand's economy, environment and society. The research conducted in this thesis has validated changes made to the dynamical core of the NZESM (i.e., the same core used in the UM) in terms of its ability to reliably simulate key characteristics of the ACV, and provides direction for additional tuning of the NZESM.

\section{Possible future research}

Given the likelihood of changes in the location, timing and influence of GWs, particularly due to differential rates of GHG forcing between tropical and polar regions (e.g., Santer et al. 2005), further research into drivers of GWs and their possible future behaviour is warranted.

Planetary waves in the stratosphere, including their generation and breaking, play an important role in controlling the shape of the ACV. Further analysis to compare resolved wave generation and breaking in the stratosphere between models and reanalyses would be expected to provide additional insight into the difficulties that models face in representing the ACV.

The identification of a bifurcated structure in the ACV has raised questions regarding approaches taken in defining the edges of the vortex and its meridional profile. Determining the edge location is important in accurately delineating the vortex region from the surf-zone - areas with fundamentally different transport dynamics. Future work could seek to develop a metric for vortex edge location that is insensitive to the internal structure of the vortex. LCSs may be one avenue to explore, given their stated effectiveness at identifying the vortex edge region (Serra et al., 2017).

Bifurcation is found to be a common feature of the ACV for specific 
months and at a range of potential temperature levels (Conway et al., 2018). There is scope for additional investigation into how the presence of a bifurcated barrier affects transport of trace gases such as ozone, which may then feed back onto vortex dynamics. Of particular interest, would be exploration of how the bifurcation tendency may be expected to change with changes in GHG concentrations and ozone recovery, and the consequences of such changes for the climate system. It appears that bifurcation is also identified by parabolic LCSs, where deformation of and comparisons of results that identify bifurcation in both $\kappa$ and LCSs would be of interest.

Detailed quantitative analysis of a wider range of diagnostic methods for detecting transport barriers and mixing would be of interest, particularly in advancing our understanding of relationships between mixing metrics and their ability to explain stratospheric dynamics and ozone depletion. This could include assessments of interannual variability and temporal trends, as well as relationships to the broader climatology. Quantitative approaches could investigate if maximum values of metrics are more or less important than average values over a range of equivalent latitudes, thereby establishing whether, for example, a wider but weaker vortex barrier presents a more or less effective transport barrier than one which is narrower but stronger. In addition, some of the metrics that were not examined in detail here could be included in subsequent assessments (e.g., FTLEs or FSLEs, mass flux, LCSs).

Additional research could build on the earlier work of Waugh (1997) and others to investigate the dynamical shape of the vortex in 3-dimensional space. While in Chapter 5 it has been established that the vortex barrier is better represented using 3-D ozone fields, this has been evaluated using a 2 -D vortex representation (i.e., the meridional profile of $\kappa$, albeit accounting for the barrier on an equivalent latitude basis). Recent research has used computer vision techniques to identify SSW occurrence in the NH vortex (Lawrence and Manney, 2017) and similar work to investigate the likelihood of increased frequency of SSWs in the ACV would be of great interest.

Another potential research direction is to assess the performance of additional GCMs and CCMs, to establish if the results found here are common across a range of models. Similarly it would be beneficial to assess how well more recent reanalyses with higher spatial and temporal resolution perform in representing the $\mathrm{ACV}$ transport barrier.

In future work it would be useful to examine the proportion of variance in model results that can be attributed to the various components that have been identified as likely contenders here (i.e., model dynamics, wave drag, ozone, and model feedbacks). This would align well with undertaking a range 
of sensitivity experiments that were not able to be undertaken here, involving changes to aspects of model physics for each of those key model components.

The expectation of further increases in computing power in the future would allow for analysis of the complex evolution, shape and strength of the vortex. Methods such as the use of LCSs to improve vortex edge definition accurately, and the ability to map and model the projected behaviour of the vortex in 3-dimensions are also expected to be areas of further development.

A lack of in-depth understanding of stratosphere-troposphere coupling makes it a clear candidate for further research - of particular interest would be enhancing our knowledge of the connections between the strength and form of the ACV and the mode of the SAM. 


\section{Appendix A}

\section{Additional metric Figures}

This appendix provides figures additional levels and for the ERA-Interim reanalysis and for the $850 \mathrm{~K}$ level to supplement the figures provided in Chapter 3 . 
1980
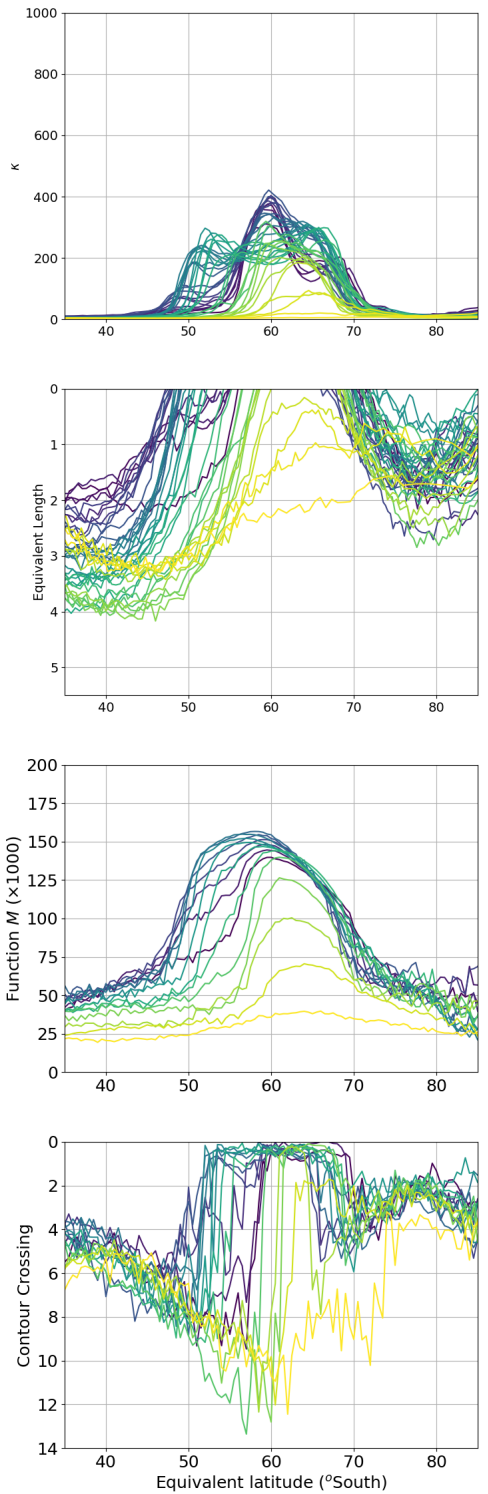

1999
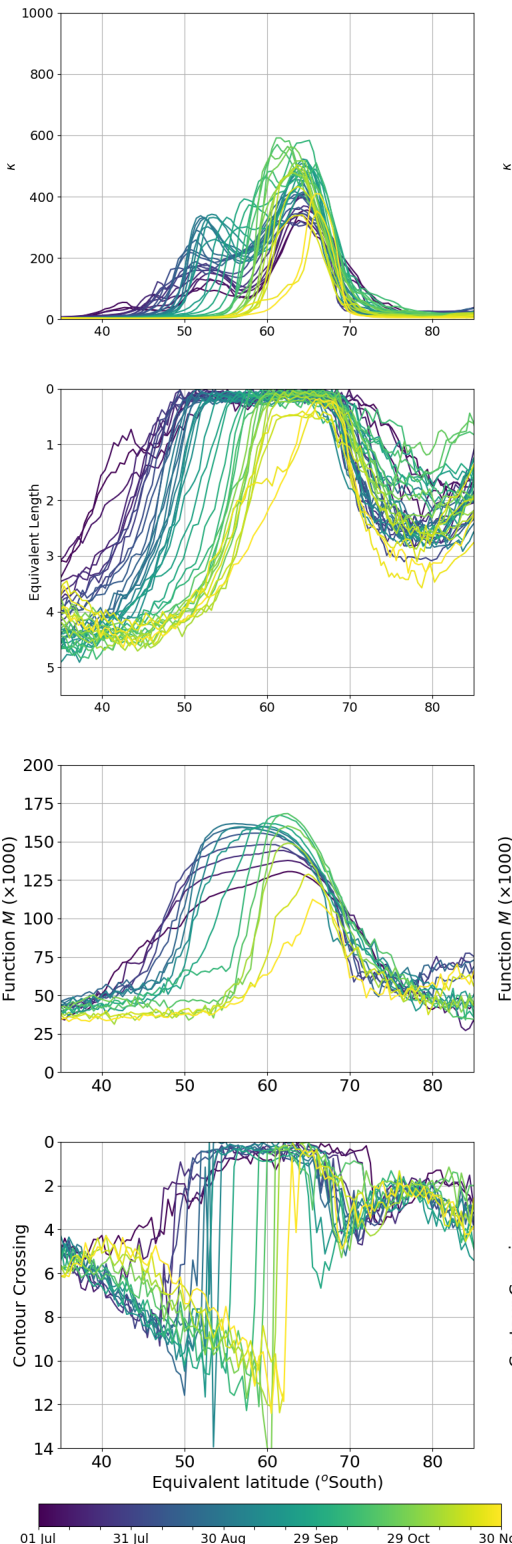

2014
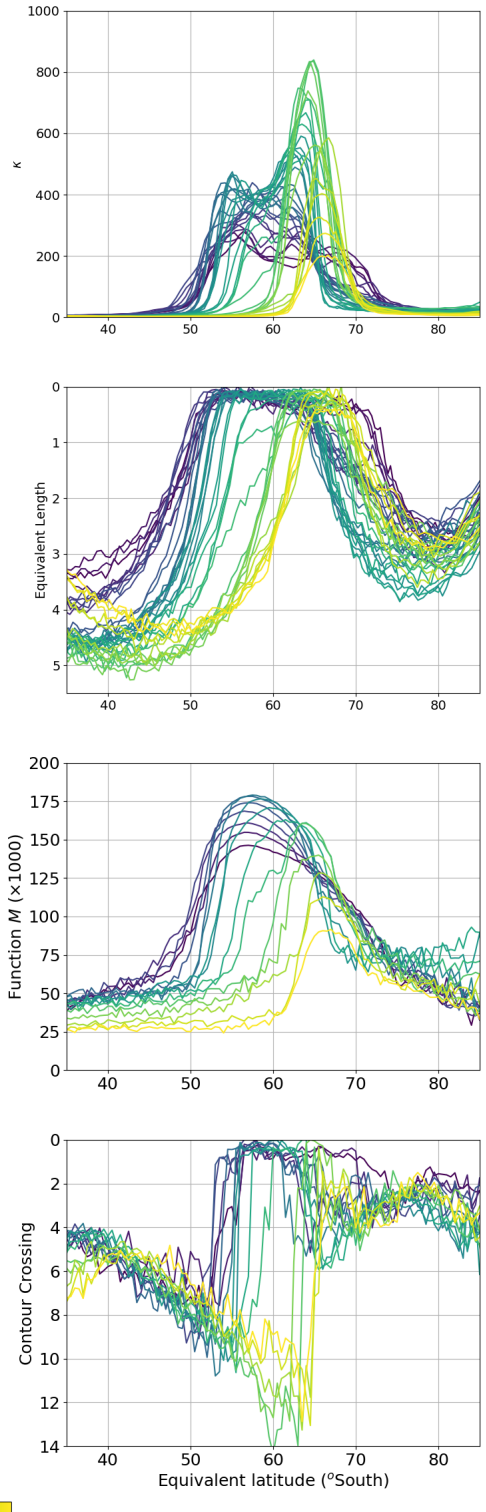

Figure A.1: As for Figure 3.9, but for ERA-Interim reanalysis. 
1980
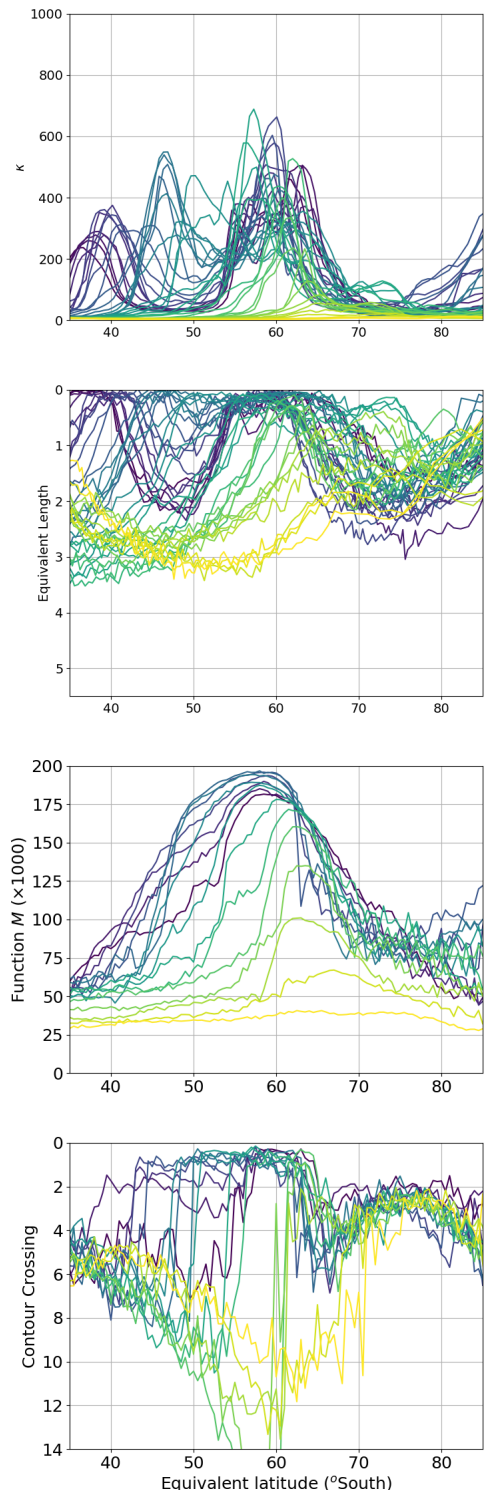

1999
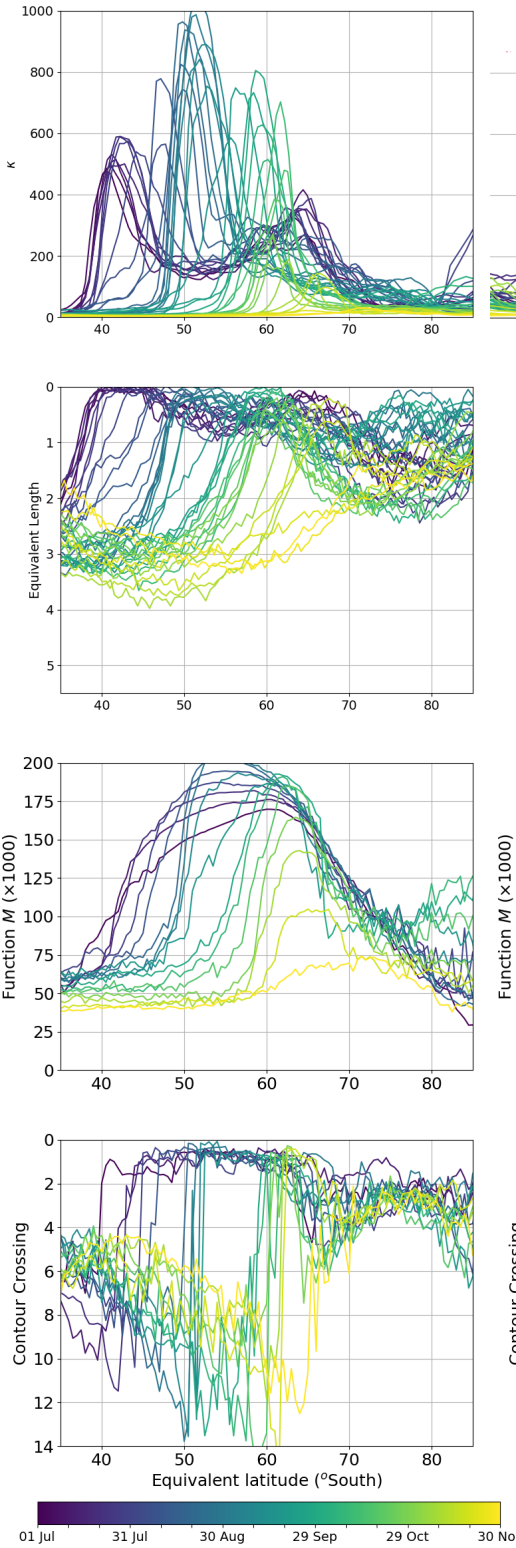

2014
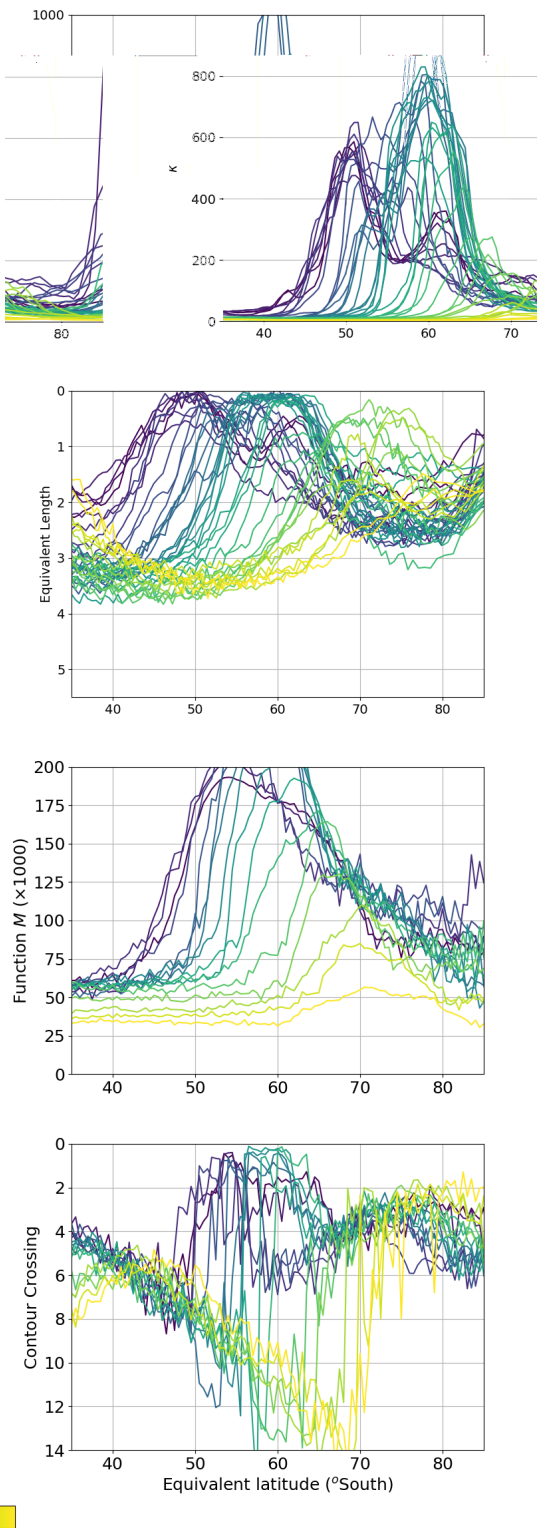

Figure A.2: As for Figure A.1, but at the $850 \mathrm{~K}$ level using ERA-Interim. 
1980
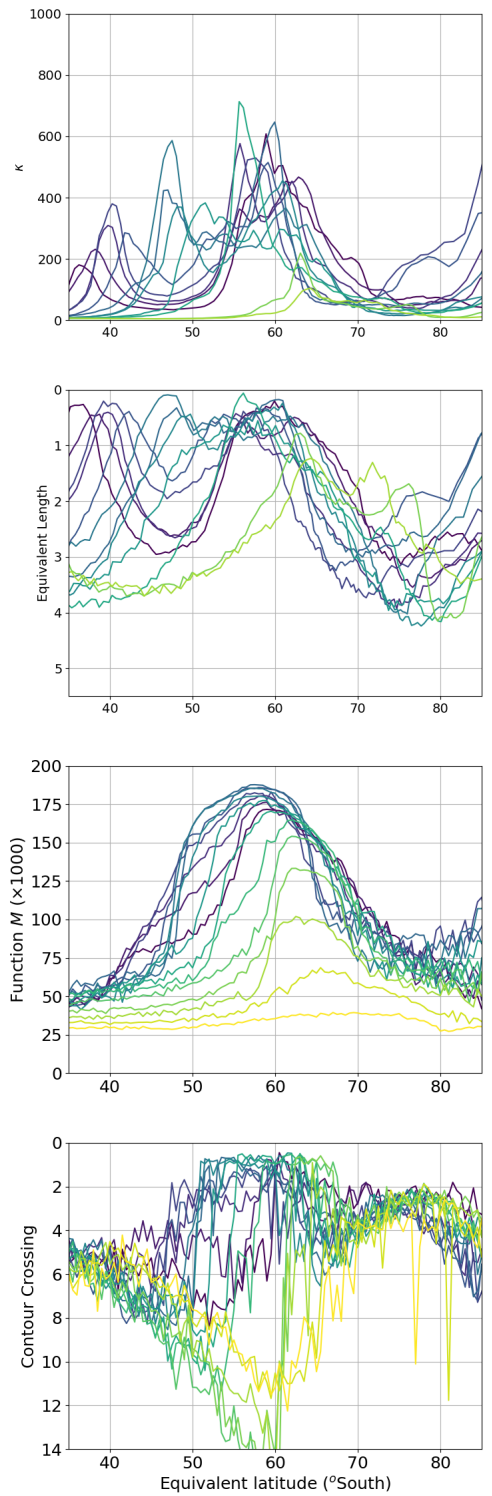

1999
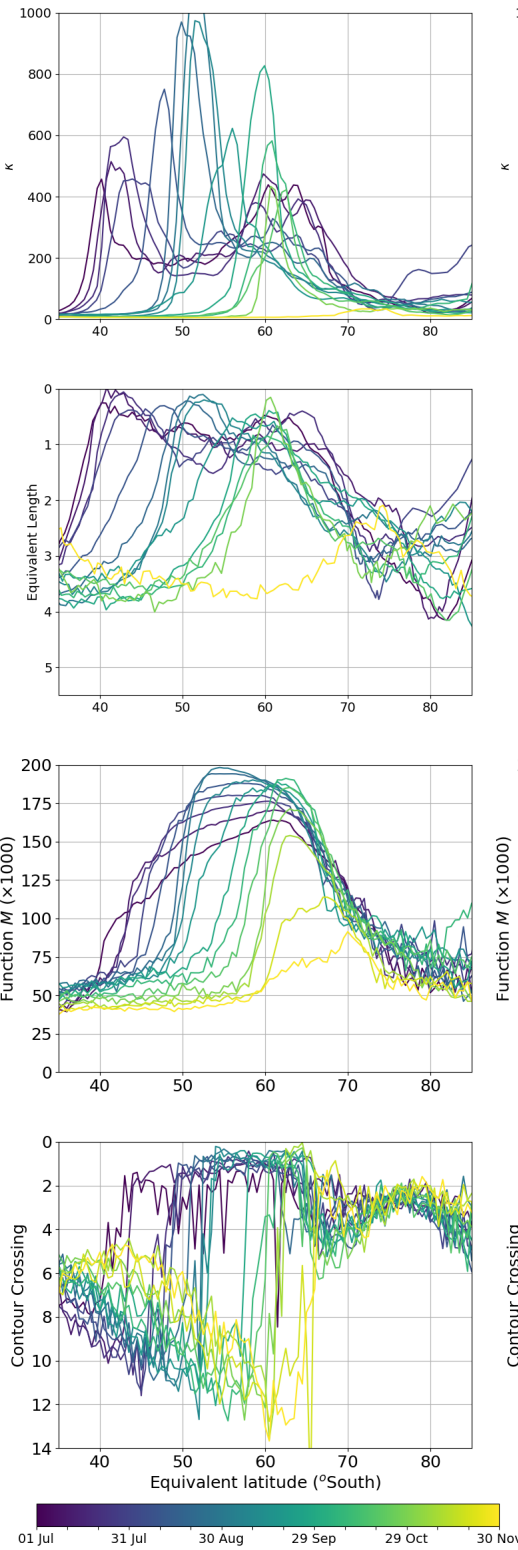

2014
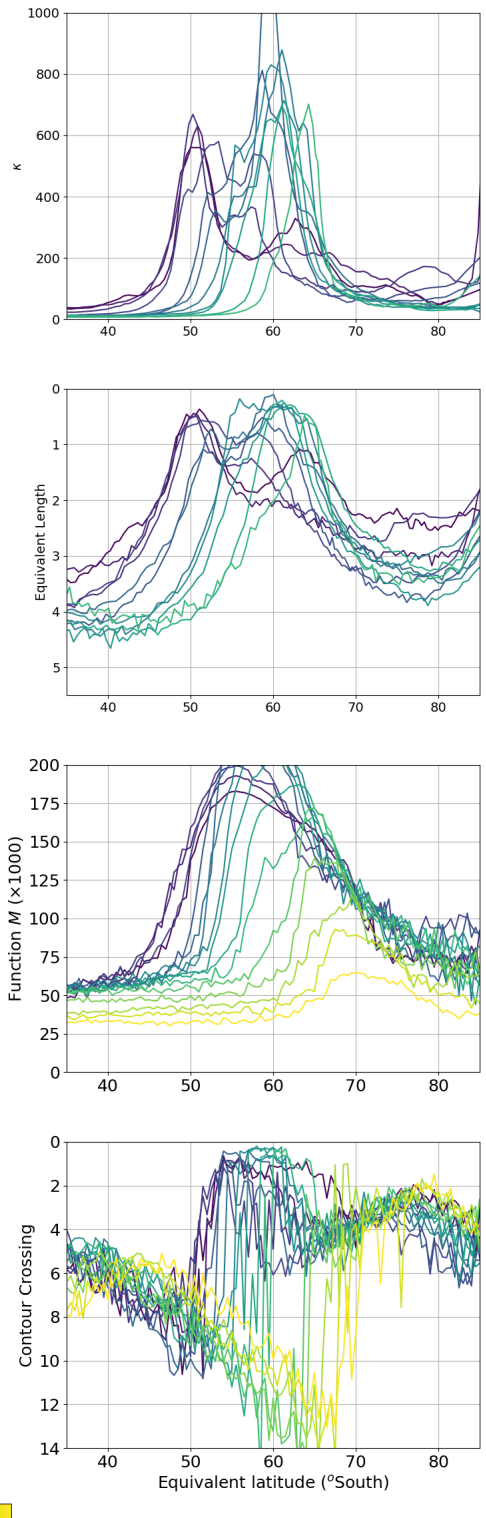

Figure A.3: As for Figure A.2 but for NCEP-CFSR reanalysis. 


\section{Appendix B}

\section{Additional UM Figures}

This appendix provides figures for additional months (July and August) and at the $850 \mathrm{~K}$ level, to supplement those provided in Chapter 4 that focussed only on September at the $550 \mathrm{~K}$ level in the Unified Model. 


\section{July: $550 \mathrm{~K}$}
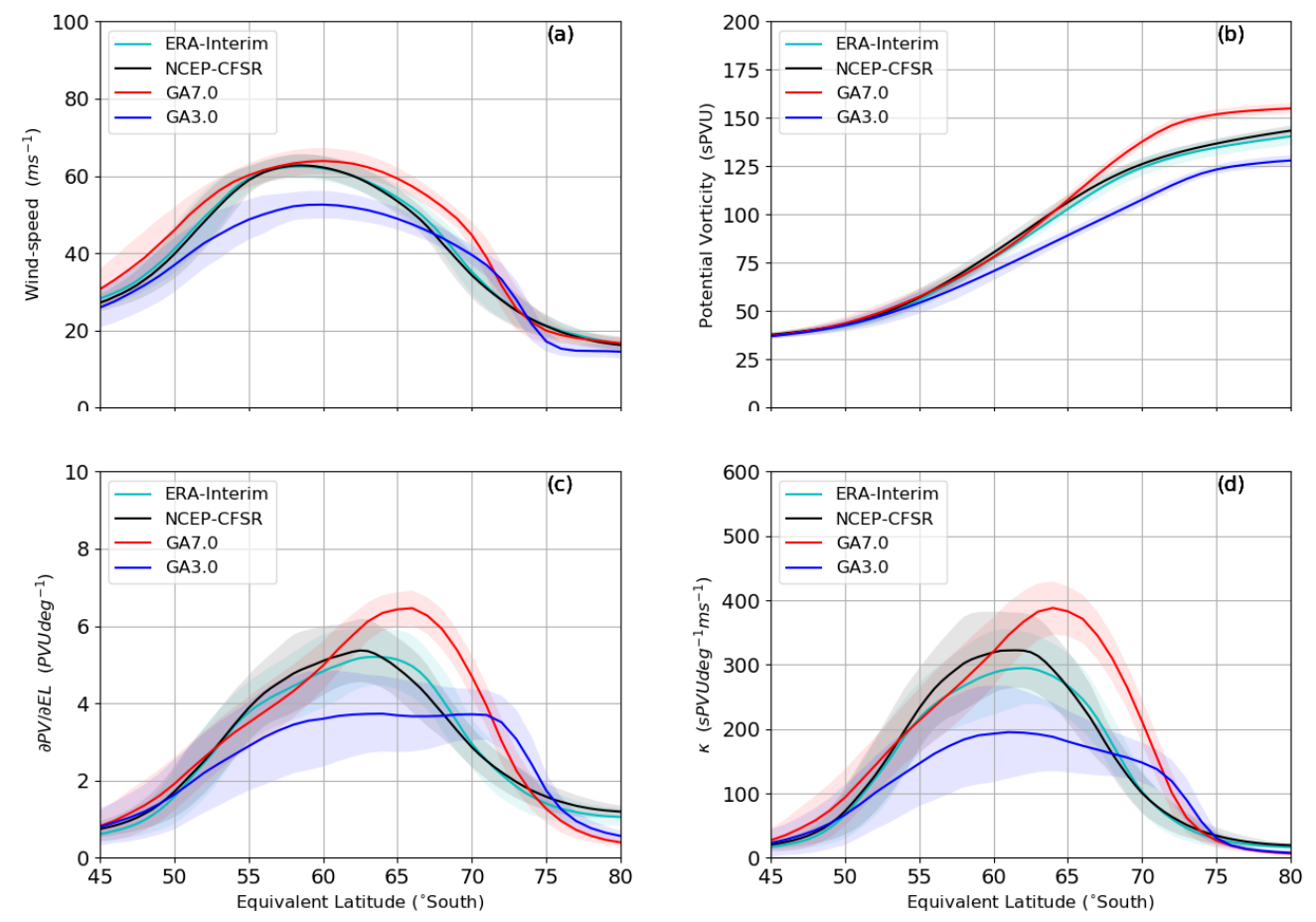

Figure B.1: As for Figure 4.1, but for July. 


\section{August: $550 \mathrm{~K}$}
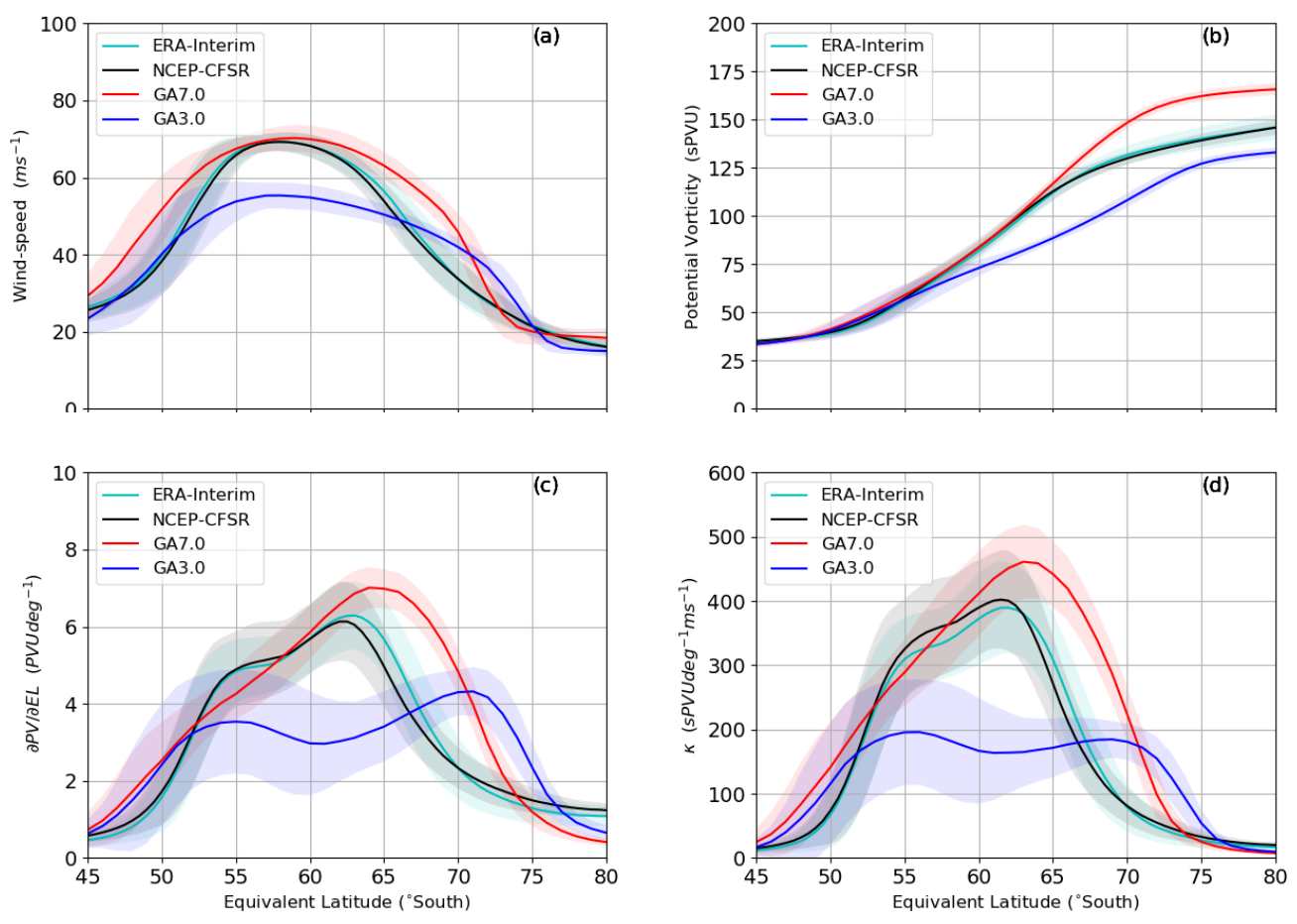

Figure B.2: As for Figure 4.1, but for August. 


\section{July: $850 \mathrm{~K}$}
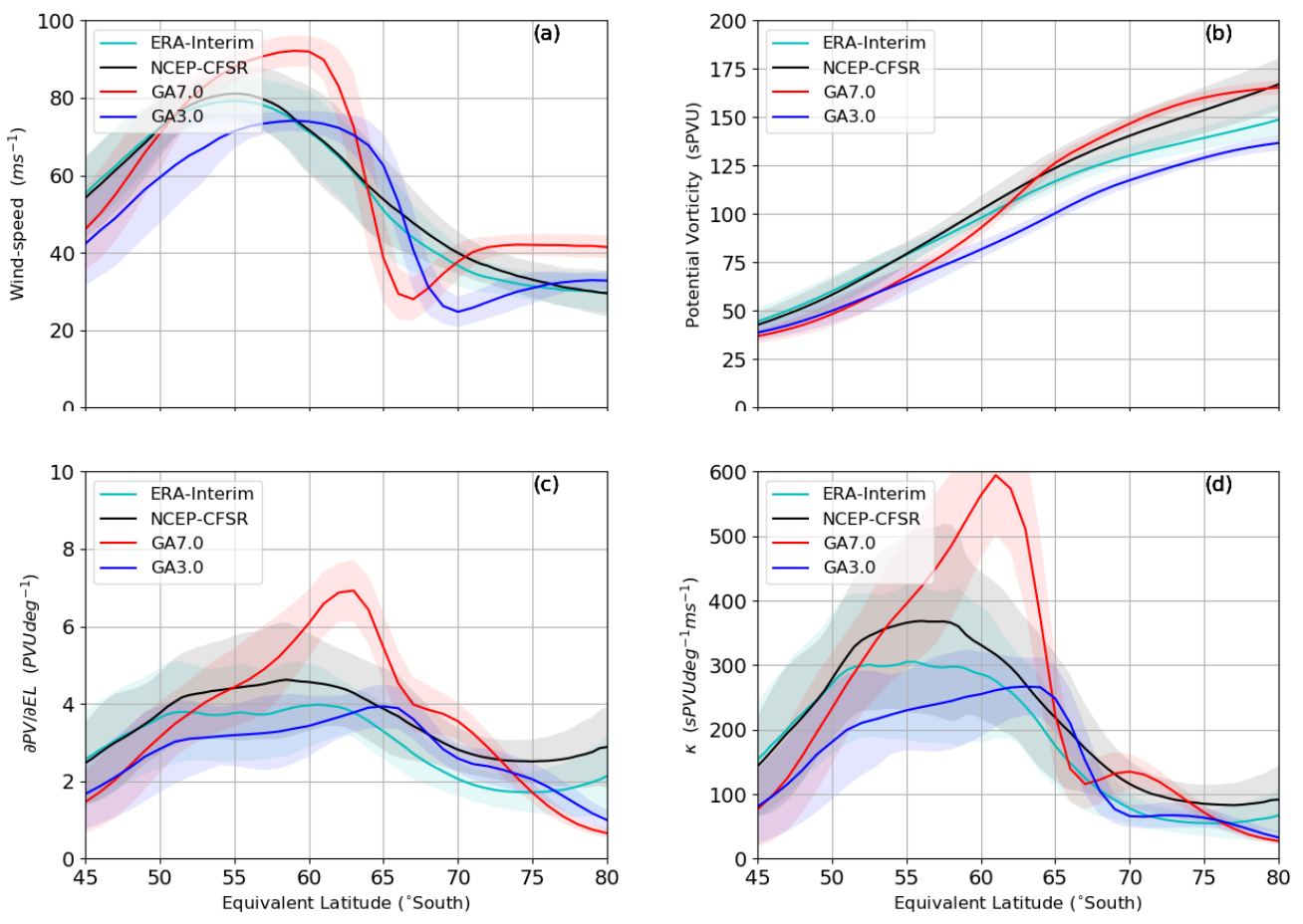

Figure B.3: As for Figure 4.1, but for July at $850 \mathrm{~K}$. 


\section{August: $850 \mathrm{~K}$}
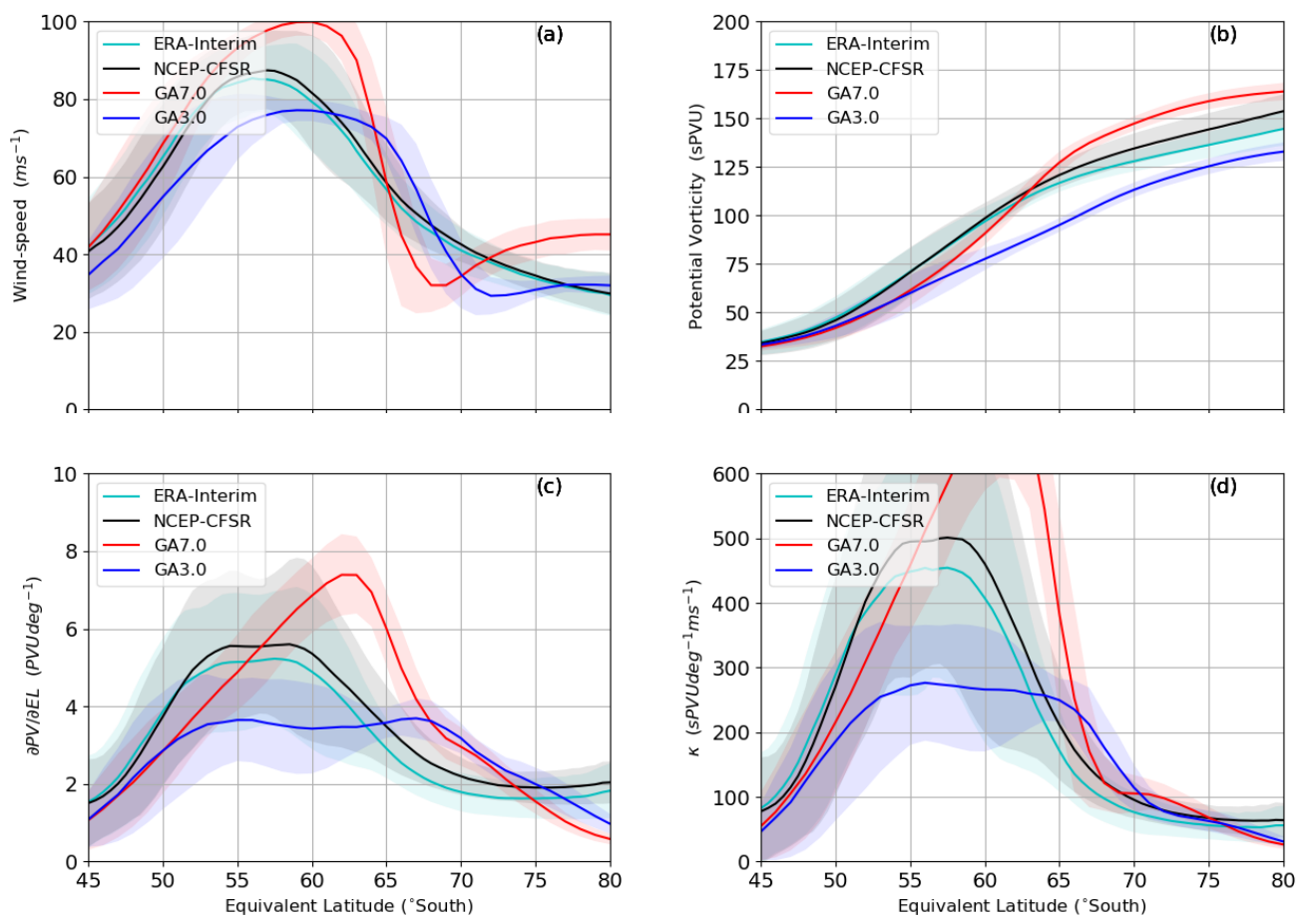

Figure B.4: As for Figure 4.1, but for August at $850 \mathrm{~K}$. 


\section{September: $850 \mathrm{~K}$}
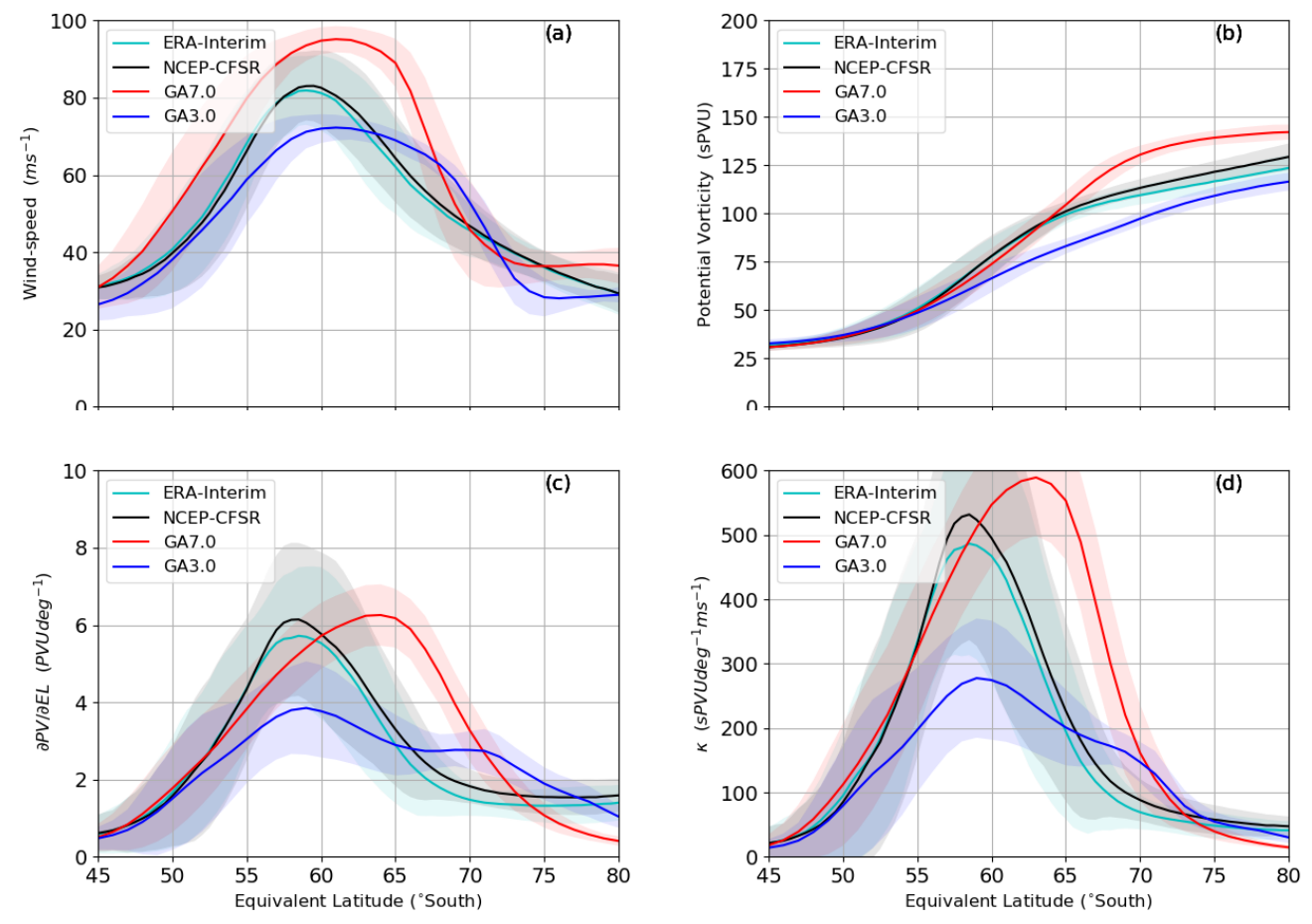

Figure B.5: As for Figure 4.1, but for September. 


\section{Appendix C}

\section{Additional SOCOLv3 Figures}

This appendix provides figures for additional months (July and August) and at the $850 \mathrm{~K}$ level, to supplement those provided in Chapter 5 that focussed only on September at the $550 \mathrm{~K}$ level in the SOCOLv3 model. 


\section{July: $550 \mathrm{~K}$}
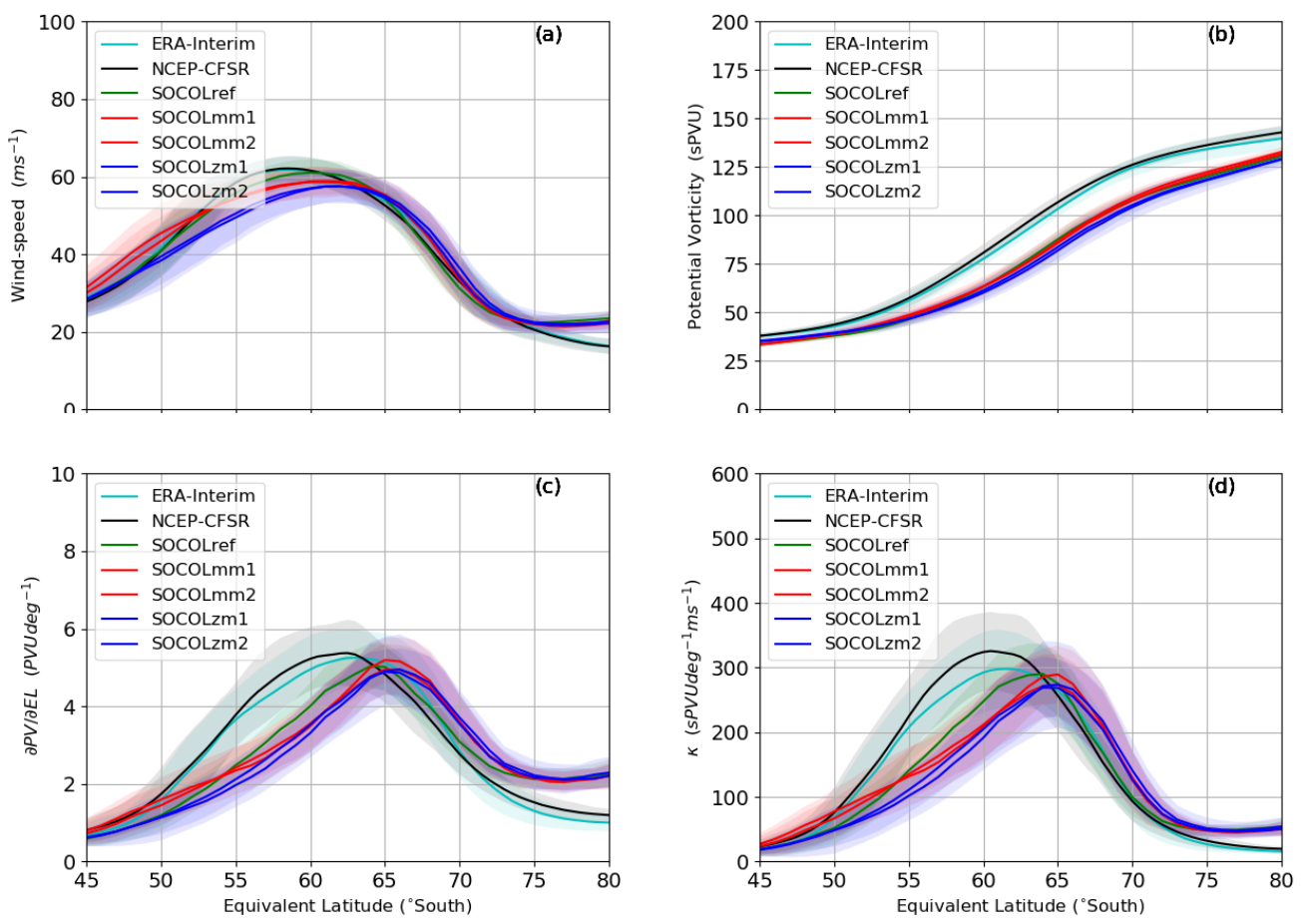

Figure C.1: As for Figure 5.1, but for July at $550 \mathrm{~K}$. 


\section{August: $550 \mathrm{~K}$}
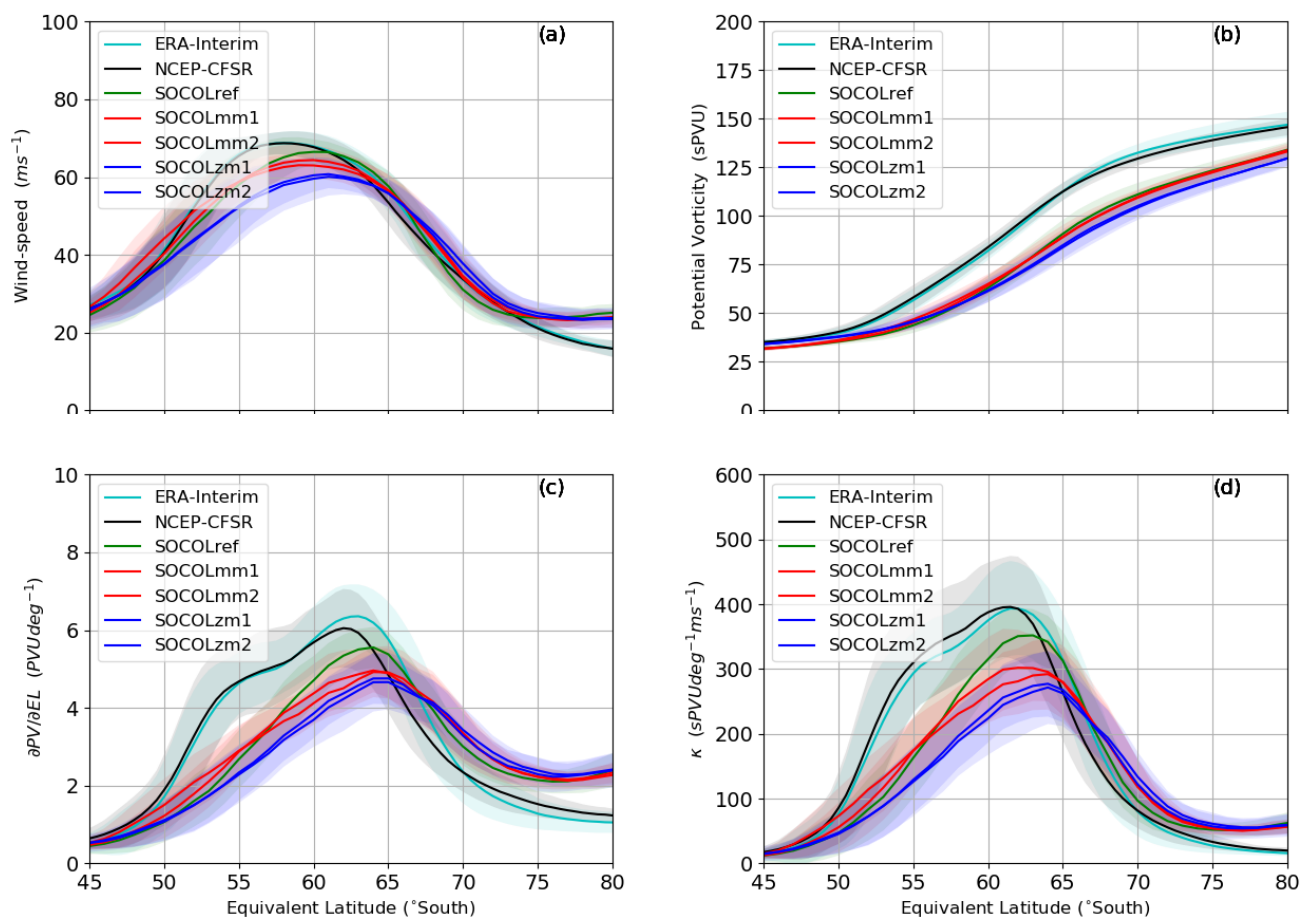

Figure C.2: As for Figure 5.1, but for August at $550 \mathrm{~K}$. 


\section{July: $850 \mathrm{~K}$}
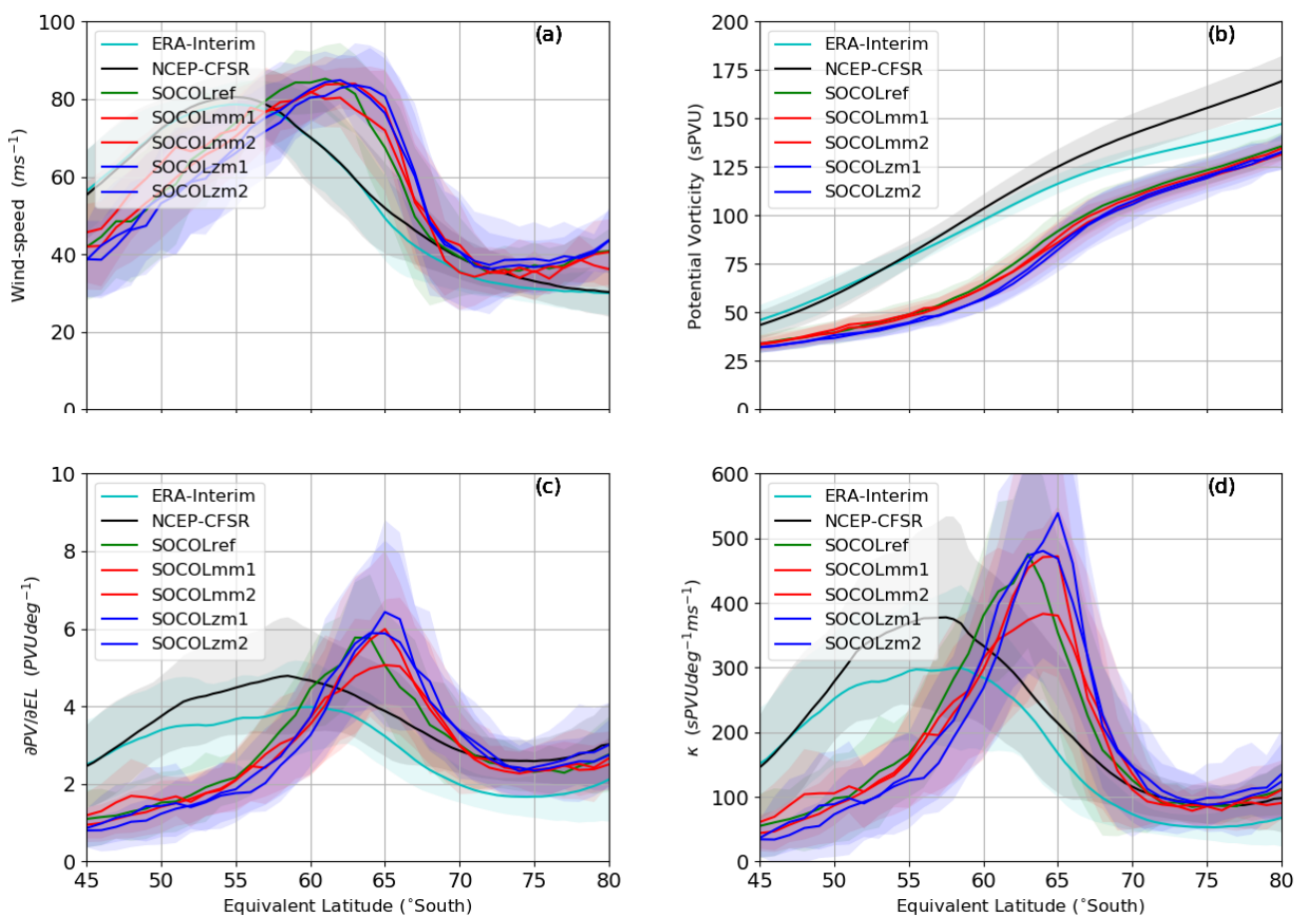

Figure C.3: As for Figure 5.1, but for July at $850 \mathrm{~K}$. 


\section{August: $850 \mathrm{~K}$}
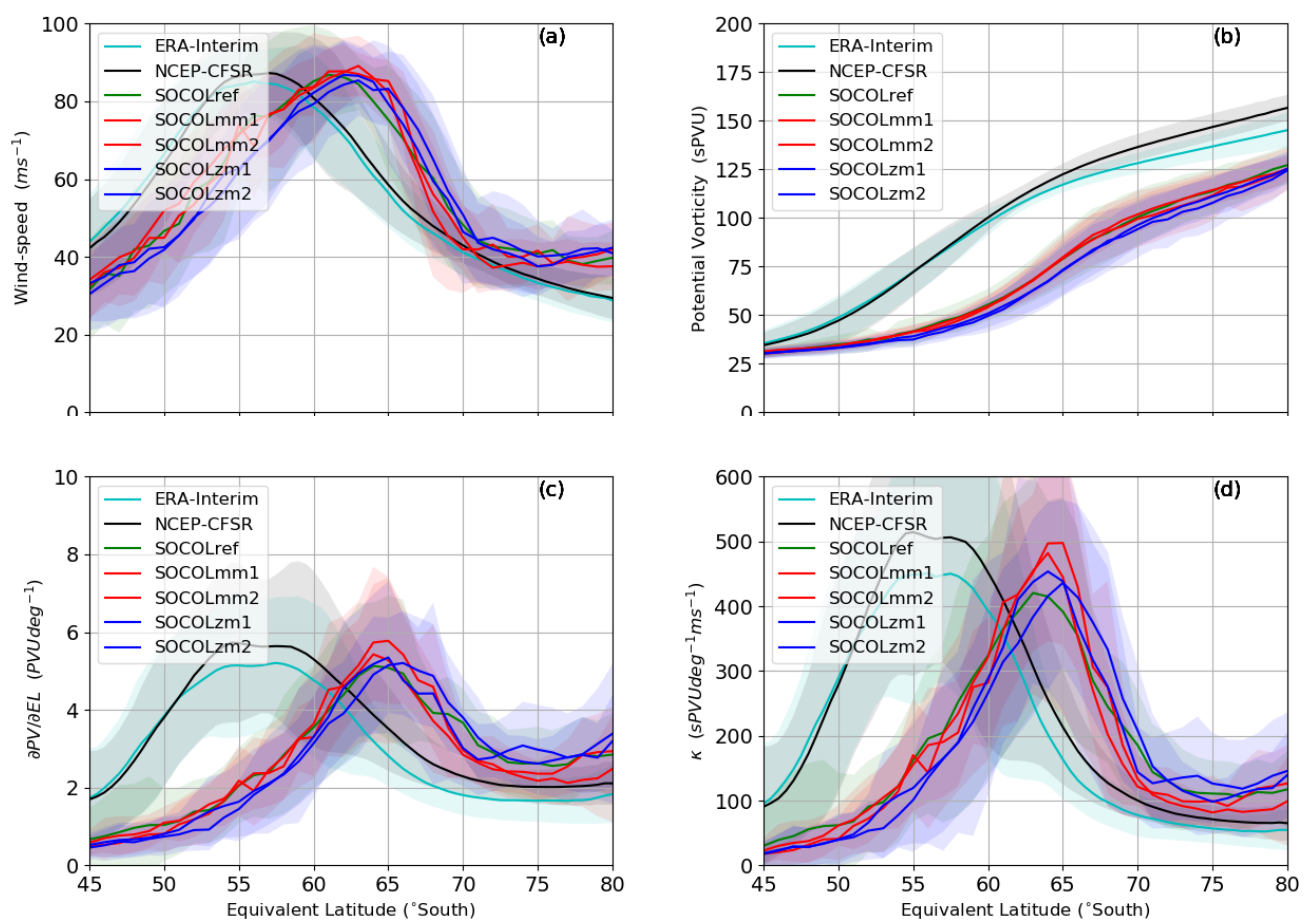

Figure C.4: As for Figure 5.1, but for August at $850 \mathrm{~K}$. 


\section{September: $850 \mathrm{~K}$}
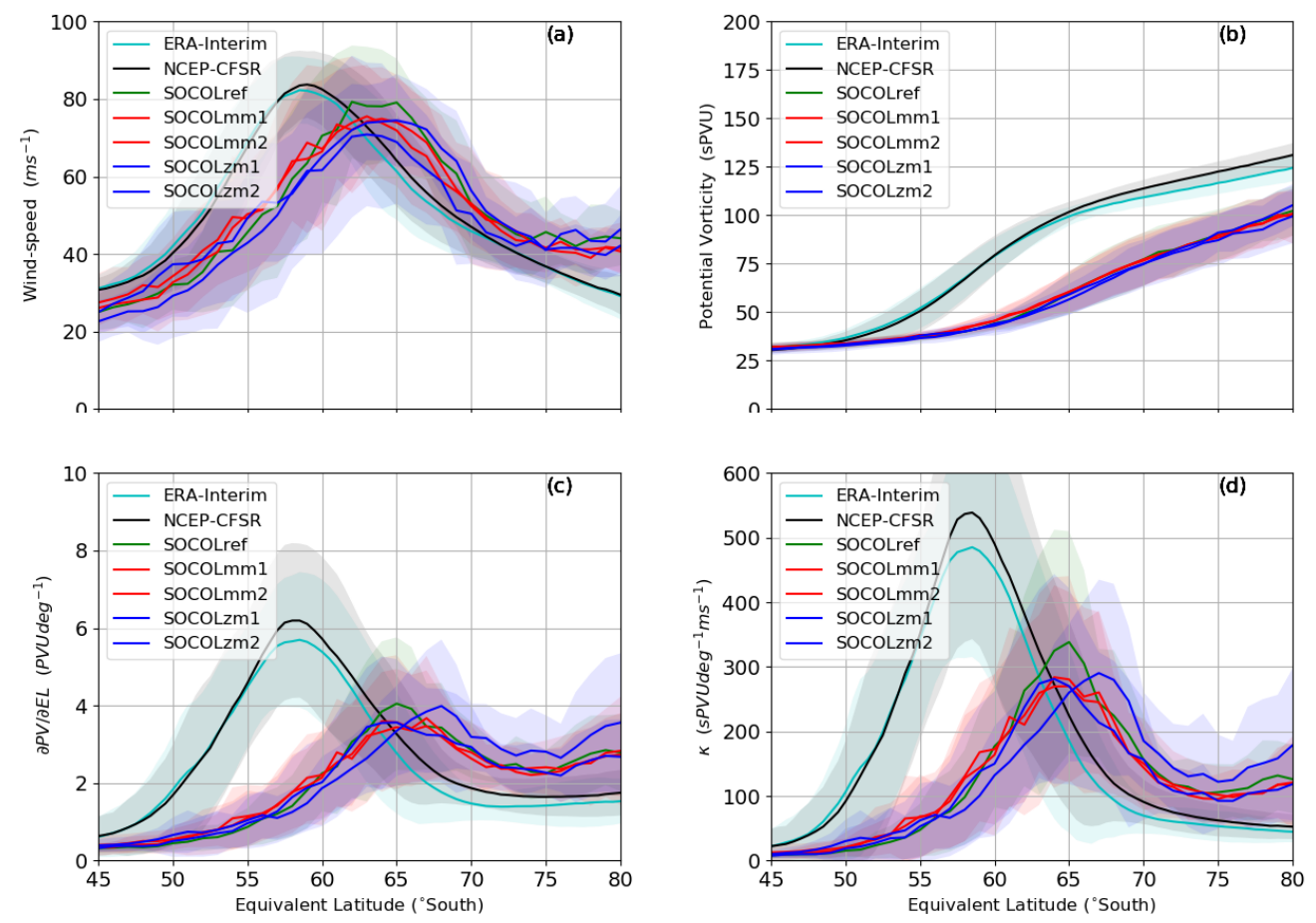

Figure C.5: As for Figure 5.1, but for September at $850 \mathrm{~K}$. 


\section{Bibliography}

Abalos, M., B. Legras, and E. Shuckburgh (2016). Interannual variability in effective diffusivity in the upper troposphere/lower stratosphere from reanalysis data. Quarterly Journal of the Royal Meteorological Society 142(697), 1847-1861.

Abram, N., R. Mulvaney, F. Vimeux, S. Phipps, J. Turner, and M. England (2014). Evolution of the Southern Annular Mode during the past millennium. Nature Climate Change 4, 564-569.

Añel, J., D. Allen, G. Sáenz, L. Gimeno, and L. de la Torre (2013). Equivalent Latitude Computation Using Regions of Interest (ROI). PLoS ONE 8(9).

Albers, J. R. and T. R. Nathan (2012). Pathways for communicating the effects of stratospheric ozone to the polar vortex: role of zonally asymmetric ozone. J. Atmos. Sci. 69, 785-801.

Alexander, M. (2010). Gravity Waves in the Stratosphere. Washington, DC: American Geophysical Union.

Allen, D., R. Bevilacqua, G. Nedoluha, C. Randall, and G. Manney (2003). Unusual stratospheric transport and mixing during the 2002 Antarctic winter. Geophysical Research Letters 30(12), 1599, doi:10.1029/2003GL017117.

Allen, D. and N. Nakamura (2001). A seasonal climatology of effective diffusivity in the stratosphere. JGR 106(D8), 7917-7935.

Allen, D. and N. Nakamura (2003). Tracer Equivalent Latitude: A Diagnostic Tool for Isentropic Transport Studies. Journal of the Atmospheric Sciences 60, 287-304.

Andrews, D., J. Holton, and C. Leovy (1987). Middle Atmosphere Dynamics. Academic Press. 
Andrews, T., J. Gregory, M. Webb, and K. Taylor (2012). Forcing, feedbacks and climate sensitivity in CMIP5 coupled atmosphere-ocean climate models. Geophysical Research Letters 39(L09712).

Aurell, E., G. Boffettaz, A. Crisantix, G. Paladin, and A. Vulpiani (1997). Predictability in the large: an extension of the concept of Lyapunov exponent. Journal of Physics A: Mathematical and General 30, 1-26.

Baldwin, M. and T. Dunkerton (1999). Propagation of the Arctic oscillation from the stratosphere to the troposphere. Journal of Geophysical Research 104(D24), 30937-30946.

Baldwin, M., L. Gray, T. Dunkerton, K. Hamilton, P. Haynes, W. Randel, J. Holton, M. Alexander, I. Hirota, T. Horinouchi, D. Jones, J. Kinnersley, C. Marquardt, K. Sato, and M. Takahashi (2001). The Quasi-Biennial Oscillation. Reviews of Geophysics 39(2), 179-229.

Bell, C., L. Gray, A. Charlton-Perez, M. Joshi, and A. Scaife (2009). Stratospheric Communication of El Niño Teleconnections to European Winter. Journal of Climate 22, 4083-4096.

Black, R. and B. McDaniel (2007). Interannual Variability in the Southern Hemisphere Circulation Organized by Stratospheric Final Warming Events. Journal of the Atmospheric Sciences 64, 2968-2974.

Boccara, G., A. Hertzog, C. Basdevant, and F. Vial (2008). Accuracy of NCEP/NCAR reanalyses and ECMWF analyses in the lower stratosphere over Antarctica in 2005. Journal of Geophysical Research 113, D20115, doi:10.1029/2008JD010116.

Bodeker, G., H. Shiona, and H. Eskes (2005). Indicators of Antarctic ozone depletion. Atmospheric Chemistry and Physics 5, 2603-2615.

Bodeker, G., H. Struthers, and B. Connor (2002). Dynamical containment of Antarctic ozone depletion. Geophysical Research Letters 29(7).

Bony, S., R. Colman, V. Kattsov, R. Allan, C. Bretherton, J.-L. Dufresne, A. Hall, S. Hallegatte, M. Holland, W. Ingram, D. Randall, B. Soden, G. Tselioudis, and M. Webb (2006). How Well Do We Understand and Evaluate Climate Change Feedback Processes? Journal of Climate 19, 3445-3482.

Bowman, K. (1993). Large-scale isentropic mixing properties of the Antarctic polar vortex from analyzed winds. Journal of Geophysical Research 98(D12), 23013-23027. 
Brown, A., S. Milton, M. Cullen, B. Golding, J. Mitchell, and A. Shelly (2012). Unified Modeling and Prediction of Weather and Climate: A 25Year Journey. Bulletin of the American Meteorological Society 93, 18651877.

Butchart, N. (2014). The Brewer-Dobson circulation. Reviews of Geophysics 52, 157-184.

Butchart, N., J. Austin, J. Knight, A. Scaife, and M. Gallani (2000). The response of the stratospheric climate to projected changes in the concentrations of well-mixed greenhouse gases from 1992 to 2051. Journal of Climate 13, 2142-2159.

Butchart, N., A. Charlton-Perez, I. Cionni, S. Hardiman, P. Haynes, K. Krüger, P. Kushner, P. Newman, S. Osprey, J. Perlwitz, M. Sigmond, L. Wang, H. Akiyoshi, J. Austin, S. Bekki, A. Baumgaertner, P. Braesicke, C. Brühl, M. Chipperfield, M. Dameris, S. Dhomse, V. Eyring, R. Garcia, H. Garny, P. Jöckel, J.-F. Lamarque, M. Marchand, M. Michou, O. Morgenstern, T. Nakamura, S. Pawson, D. Plummer, J. Pyle, E. Rozanov, J. Scinocca, T. Shepherd, K. Shibata, D. Smale, H. Teyssèdre, W. Tian, D. Waugh, and Y. Yamashita (2011). Multimodel climate and variability of the stratosphere. Journal of Geophysical Research 116, D05102, doi:10.1029/2010JD014995.

Butchart, N. and E. Remsberg (1986). The area of the stratospheric polar vortex as a diagnostic for tracer transport on an isentropic surface. Journal of the Atmospheric Sciences 43(13), 1319-1339.

Cameron, C., G. Bodeker, J. Conway, L. Revell, S. Andrea, and J. Renwick (sbmt). Comparing the effects of the dimensionality of prescribed and interactive ozone on the fidelity of the Antarctic stratospheric vortex in a global climate model. Geophysical Research Letters.

Cameron, C., G. Bodeker, J. Conway, S. Stuart, and J. Renwick (2019). Simulating the Antarctic stratospheric vortex transport barrier: comparing the Unified Model to reanalysis. Climate Dynamics 53(1-2), 441-452.

Cameron, C., J. Conway, G. Bodeker, and J. Renwick (prep). A comparison of stratospheric mixing metrics in the vicinity of the Antarctic circumpolar vortex. Atmospheric Chemistry and Physics.

Checa-Garcia, R., M. Hegglin, D. Kinnison, D. Plummer, and K. Shine (2018). Historical Tropospheric and Stratospheric Ozone Radiative Forcing Using the CMIP6 Database. Geophysical Research Letters 45(7). 
Chen, P. (1994). The permeability of the Antarctic vortex edge. Journal of Geophysical Research 99(D10), 20563-20571.

Chiodo, G. and L. M. Polvani (2016). Reduced Southern Hemispheric circulation responseto quadrupled CO2 due to stratosphericozone feedback. Geophysical Research Letters 44, 465-474.

Codron, F. (2005). Relation between Annular Modes and the Mean State: Southern Hemisphere Summer. Journal of Climate 18, 320-330.

Cohen, N., E. Gerber, and O. Bühler (2014). What Drives the Brewer-Dobson Circulation? Journal of the Atmospheric Sciences 71, $3837-3855$.

Conway, J., G. Bodeker, and C. Cameron (2018). Bifurcation of potential vorticity gradients across the Southern Hemisphere stratospheric polar vortex. Atmospheric Chemistry and Physics 18, 8065-8077.

Conway, J., G. Bodeker, D. Waugh, D. Murphy, C. Cameron, and J. Lewis (2019). Using Project Loon super-pressure balloon observations to investigate the inertial peak in the intrinsic wind spectrum in the mid-latitude stratosphere. Journal of Geophysical Research Submitted, December 2018.

Crook, J., N. Gillett, and S. Keeley (2008). Sensitivity of Southern Hemisphere climate to zonal asymmetry in ozone. Geophysical Research Letters 35, L07806, doi:10.1029/2007GL032698.

Dahlberg, S. and K. Bowman (1995). Isentropic mixing in the Arctic stratosphere during the 1992-1993 and 1993-1994 winters. Geophysical Research Letters 22(10), 1237-1240.

Davies, T., M. Cullen, A. Malcolm, M. Mawson, A. Staniforth, A. White, and N. Wood (2005). A new dynamical core for the Met Office's global and regional modelling of the atmosphere. Quarterly Journal of the Royal Meteorological Society 131(608), 1759-1782.

de la Cámara, A., C. Mechoso, A. Mancho, E. Serrano, and Y. Ide (2013). Isentropic Transport within the Antarctic Polar-Night Vortex: Rossby Wave Breaking Evidence and Lagrangian Structures. Journal of the Atmospheric Sciences 70, 2982-3001.

Dee, D., S. Uppala, A. Simmons, P. Berrisford, P. Poli, S. Kobayashi, U. Andrae, M. A. Balmaseda, G. Balsamo, P. Bauer, P. Bechtold, A. C. M. 
Beljaars, L. van de Berg, J. Bidlot, N. Bormann, C. Delsol, R. Dragani, M. Fuentes, A. J. Geer, L. Haimberger, S. B. Healy, H. Hersbach, E. V. Hólm, L. Isaksen, P. Kållberg, M. Köhler, M. Matricardi, A. P. McNally, B. M. Monge-Sanz, J. Morcrette, B. Park, C. Peubey, P. de Rosnay, C. Tavolato, J. Thépaut, and F. Vitart (2011). The ERA-Interim reanalysis: Configuration and performance of the data assimilation system. Quart. J. R. Meteorol. Soc. 137, 553-597.

Dennison, F., A. Mcdonald, and O. Morgenstern (2015). The effect of ozone depletion on the Southern Annular Mode and stratosphere-troposphere coupling. Journal of Geophysical Research 120, 6305-6312.

Dennison, F., A. McDonald, and O. Morgenstern (2017). The evolution of zonally asymmetric austral ozone in a chemistry climate model. Atmospheric Chemistry and Physics 17(22), 14075-14084.

Deser, C., A. Phillips, V. Bourdette, and H. Teng (2012). Uncertainty in climate change projections: the role of internal variability. Climate Dynamics 38(527-46).

Dritschel, D. (1989). Contour dynamics and contour surgery: Numerical algorithms for extended, high-resolution modelling of vortex dynamics in two-dimensional, inviscid, incompressible flows. Computer Physics Reports 10, 77-146.

Dunkerton, T. (1997). The role of gravity waves in the quasibiennial oscillation. J. Geophys. Res. 102(26).

Egorova, T., E. Rozanov, V. Zubov, E. Manzini, W. Schmutz, and T. Peter (2005). Chemistry-climate model SOCOL: a validation of the present-day climatology. Atmospheric Chemistry and Physics 5, 1557-1576.

Eyring, V., J. M. Arblaster, I. Cionni, J. Sedláček, J. Perlwitz, P. J. Young, S. Bekki, D. Bergmann, P. Cameron-Smith, W. J. Collins, G. Faluvegi, K. D. Gottschaldt, L. W. Horowitz, D. E. Kinnison, J. F. Lamarque, D. R. Marsh, D. Saint-Martin, D. T. Shindell, K. Sudo, S. Szopa, and S. Watanabe (2013). Long-term ozone changes and associated climate impacts in CMIP5 simulations. Journal of Geophysical Research 118(10), 5029-5060.

Eyring, V., N. Butchart, D. Waugh, H. Akiyoshi, J. Austin, S. Bekki, G. Bodeker, B. Boville, C. Brühl, M. Chipperfield, E. Cordero, M. Dameris, M. Deushi, V. Fioletov, S. Frith, R. Garcia, A. Gettelman, M. Giorgetta, V. Grewe, L. Jourdain, D. Kinnison, E. Mancini, E. Manzini, M. Marchand, D. Marsh, T. Nagashima, P. Newman, J. Nielsen, S. Pawson, 
G. Pitari, D. Plummer, E. Rozanov, M. Schraner, T. Shepherd, K. Shibata, R. Stolarski, H. Struthers, W. Tian, and M. Yoshiki (2006). Assessment of temperature, trace species, and ozone in chemistry-climate model simulations of the recent past. Journal of Geophysical Research 111, D22308, doi:10.1029/2006JD007327.

Farazmand, M., D. Blazevski, and G. Haller (2014). Shearless transport barriers in unsteady two-dimensional flows and maps. Physica D: Nonlinear Phenomena 278-279, 44-57.

Friedrich, L., A. Mcdonald, G. Bodeker, K. Cooper, J. Lewis, and A. Paterson (2017). A comparison of Loon balloon observations and stratospheric reanalysis products. Atmospheric Chemistry and Physics 17, 855-866.

Fujiwara, M., J. Wright, G. Manney, L. Gray, J. Anstey, T. Birner, S. Davis, E. Gerber, V. Harvey, M. Hegglin, C. Homeyer, J. Knox, K. Krüger, A. Lambert, C. Long, P. Martineau, A. Molod, B. Monge-Sanz, M. Santee, S. Tegtmeier, S. Chabrillat, D. Tan, D. Jackson, S. Polavarapu, G. Compo, R. Dragani, W. Ebisuzaki, Y. Harada, C. Kobayashi, W. McCarty, K. Onogi, S. Pawson, A. J. Simmons, K. Wargan, J. Whitaker, and C. Zou (2017). Introduction to the SPARC Reanalysis Intercomparison Project (S-RIP) and overview of the reanalysis systems. Atmospheric Chemistry and Physics 17, 1417-1452.

Fyfe, J., G. Boer, and G. Flato (1999). The Arctic and Antarctic oscillations and their projected changes under global warming. Geophysical Research Letters 26(11), 1601-1604.

Gabriel, A., D. Peters, I. Kirchner, and H.-F. Graf (2007). Effect of zonally asymmetric ozone on stratospheric temperature and planetary wave propagation. Geophysical Research Letters 34, L06807, doi:10.1029/2006GL028998.pdf.

Garcia, R., A. Smith, D. Kinnison, A. C'amara, and D. Murphy (2017). Modification of the gravity wave parameterization in the Whole Atmosphere Community Climate Model: motivation and results. Journal of the Atmospheric Sciences 74(1), 275-291.

Garny, H., G. Bodeker, and M. Dameris (2007). Trends and variability in stratospheric mixing: 1979-2005. Atmospheric Chemistry and Physics Discussions 7, 6189-6228. 
Garny, H., V. Grewe, M. Dameris, G. Bodeker, and A. Stenke (2011). Attribution of ozone changes to dynamical and chemical processes in CCMs and CTMs. Geoscientific Model Development 4, 271-286.

Gerber, E. (2012). Stratospheric versus Tropospheric Control of the Strength and Structure of the Brewer-Dobson Circulation. Journal of the Atmospheric Sciences.

Gillett, N., J. Scinocca, D. Plummer, and M. Reader (2009). Sensitivity of climate to dynamically-consistent zonal asymmetries in ozone. Geophysical Research Letters 36, L10809, doi:10.1029/2009GL037246.

Gong, D. and S. Wang (1999). Definition of Antarctic oscillation index. Geophysical Research Letters 26(4), 459-462.

Goosse, H., J. Kay, K. Armour, A. Bodas-Salcedo, H. Chepfer, D. Docquier, A. Jonko, P. Kushner, O. Lecomte, F. Massonnet, H. Park, F. Pithan, G. Svensson, and M. Vancoppenolle (2018). Quantifying climate feedbacks in polar regions. Nature communications, 9(1).

Grytsai, A., O. Evtushevsky, O. Agapitov, A. Klekociuk, and G. Milinevsky (2007). Structure and long-term change in the zonal asymmetry in Antarctic total ozone during spring. Annales Geophysicae 25, 361-374.

Grytsai, A., A. Klekociuk, G. Milinevsky, O. Evtushevsky, and K. Stone (2017). Evolution of the eastward shift in the quasi-stationary minimum of the Antarctic total ozone column. Atmospheric Chemistry and Physics 17, $1741-1758$.

Guha, A., C. Mechoso, C. Konor, and R. Heikes (2016). Modeling Rossby wave breaking in the southern spring stratosphere. Journal of the Atmospheric Sciences 73(1), 393.

Haigh, J. and H. Roscoe (2009). The Final Warming Date of the Antarctic Polar Vortex and Influences on its Interannual Variability. Journal of Climate 22, 5810-5819.

Haller, G. (2005). An objective definition of a vortex. J. Fluid Mech. 525, $1-26$.

Haller, G. (2015). Lagrangian Coherent Structures. Annual Review of Fluid Mechanics 47, 137-162.

Haller, G. and F. Beron-Vera (2012). Geodesic theory of transport barriers in two-dimensional flows. Physica D 241, 1680-1702. 
Hardiman, S., N. Butchart, F. O'Connor, and S. Rumbold (2017). The Met Office HadGEM3-ES chemistry-climate model: evaluation of stratospheric dynamics and its impact on ozone. Geoscientific Model Development 10(3), 1209-1232.

Hartmann, D., L. Heidt, M. Loewenstein, J. Podoloske, J. Vedder, W. Starr, and S. Strahan (1989). Transport into the south polar vortex in early spring. Journal of Geophysical Research 94(D14), 16779-16795.

Harvey, V., C. Randall, and M. Hitchman (2009). Breakdown of potential vorticity-based equivalent latitude as a vortex-centered coordinate in the polar winter mesosphere. Journal of Geophysical Research 114, D22105, doi:10.1029/2009JD012681.

Hassler, B., G. Bodeker, S. Solomon, and P. Young (2011). Changes in the polar vortex: Effects on Antarctic total ozone observations at various stations. Geophysical Research Letters 38, L01805, doi:10.1029/2010GL045542.

Haynes, P., C. Marks, M. McIntyre, T. Shepherd, and K. Shine (1991). On the 'downward control' of extratropical diabatic circulations by eddyinduced mean zonal forces. Journal of the Atmospheric Sciences 48(4), 651-678.

Haynes, P. and M. McIntyre (1987). On the evolution of vorticity and potential vorticity in the presence of diabtic and frictional or other forces. Journal of the Atmospheric Sciences 44(5), 828-841.

Haynes, P. and E. Shuckburgh (2000). Effective diffusivity as a diagnostic of atmospheric transport 1. Stratosphere. Journal of Geophysical Research 105(D18), 22777-22794.

Hendricks, E., J. Doyle, S. Eckermann, Q. J., and A. Reinecke (2014). What Is the Source of the Stratospheric Gravity Wave Belt in Austral Winter? Journal of the Atmospheric Sciences 71(5).

Holton, J. (2004). An Introduction to Dynamic Meteorology. USA: Elsevier Academic Press.

Holton, J. and J. Austin (1991). The influence of the equatorial QBO on sudden stratospheric warmings. Journal of the Atmospheric Sciences 48(4), 607-618. 
Holton, J., P. Haynes, M. McIntyre, A. Douglass, R. Rood, and L. Pfister (1995). Stratosphere-troposphere exchange. Reviews of Geophysics 33(4), 403-439.

Holton, J. and H.-C. Tan (1980). The influence of the quasi-biennial osciallation on the global circulation at $50 \mathrm{mb}$. Journal of the Atmospheric Sciences 37, 2200-2208.

Hoskins, B. (2015). Potential Vorticity and the PV Perspective. Advances in Atmospheric Sciences 32, 2-9.

Hoskins, B., M. McIntyre, and A. Robertson (1985). On the use and significance of isentropic potential vorticity maps. Quarterly Journal of the Royal Meteorological Society 111, 877-946.

Huth, R. and P. Canziani (2003). Classification of hemispheric monthly mean stratospheric potential vorticity fields. Annales Geophysicae 21, 805-817.

Ialongo, I., V. Sofieva, N. Kalakoski, J. Tamminen, and E. Kyrola (2012). Ozone zonal asymmetry and planetary wave characterization during Antarctic spring. Atmospheric Chemistry and Physics 12, 2603-2614.

Ivy, D., C. Hilgenbrink, D. Kinnison, R. Plumb, A. Sheshadri, S. Solomon, and D. Thompson (2017). Observed Changes in the Southern Hemispheric Circulation in May. Journal of Climate.

Jones, J., S. Gille, H. Goosse, N. Abram, P. Canziani, D. Charman, K. Clem, X. Crosta, C. de Lavergne, I. Eisenman, M. England, R. Fogt, L. Frankcombe, G. Marshall, V. Masson-delmotte, A. Morrison, A. Orsi, M. Raphael, J. Renwick, D. Schneider, G. Simpkins, E. Steig, B. Stenni, D. Swingedouw, and T. Vance (2016). Assessing recent trends in highlatitude Southern Hemisphere surface climate. Nature Climate Change 6, 917-926.

Joseph, B. and B. Legras (2002). Relation between kinematic boundaries, stirring, and barriers for the Antarctic polar vortex. Journal of the Atmospheric Sciences 59, 1198-1212.

Juckes, M. and M. McIntyre (1987). A high-resolution one-layer model of breaking planetary waves in the stratosphere. Nature 328, 590-596.

Karoly, D., R. Plumb, and M. Ting (1989). Examples of the horizontal propagation of quasi-stationary waves. Journal of the Atmospheric Sciences 46(18), 2802-2811. 
Karpechko, A., D. Maraun, and V. Eyring (2013). Improving Antarctic total ozone projections by a process-oriented multiple diagnostic ensemble regression. Journal of the Atmospheric Sciences 70(12), 3959-3976.

Kidston, J., A. Scaife, S. Hardiman, D. Mitchell, N. Butchart, M. Baldwin, and L. Gray (2015). Stratospheric influence on tropospheric jet streams, storm tracks and surface weather. Nature Geoscience 8.

Knox, J. (1998). On Converting Potential Temperature to Altitude in the Middle Atmosphere. EOS 79(31).

Labitzke, K. and H. Van Loon (1999). The Stratosphere: Phenomena, History, and Relevance. Springer.

Lait, L. (1994). An alternative form for potential vorticity. Journal of the Atmospheric Sciences 51(12), 1754-1759.

Lawrence, Z. and G. Manney (2017). Characterizing Stratospheric Polar Vortex Variability With Computer Vision Techniques. JGR 123(3), 15101535 .

Leovy, C., C.-R. Sun, M. Hitchman, E. Remsberg, J. Russell, L. Gordley, J. Gille, and L. Lyjak (1985). Transport of ozone in the middle stratosphere: evidence for planetary wave breaking. Journal of the Atmospheric Sciences 42(3), 230-244.

Limpasuvan, V., D. Hartmann, D. Thompson, K. Jeev, and Y. Yung (2005). Stratosphere-troposphere evolution during polar vortex intensification. Journal of Geophysical Research 110, D24101, doi:10.1029/2005JD006302.

Linz, M., R. Plumb, E. Gerber, F. Haenel, G. Stiller, D. Kinnison, A. Ming, and J. Neu (2017). The strength of the meridional overturning circulation of the stratosphere. Nature Geoscience 10, 663-667.

Madrid, J. and A. Mancho (2009). Distinguished trajectories in time dependent vector fields. Chaos 19(13).

Manney, G. and Z. Lawrence (2016). The major stratospheric final warming in 2016: dispersal of vortex air and termination of Arctic chemical ozone loss. Atmospheric Chemistry and Physics 16, 15371-15396.

Manney, G., J. Sabutis, D. Allen, W. Lahoz, A. Scaife, C. Randall, S. Pawson, B. Naujokat, and R. Swinbank (2005). Simulations of Dynamics and Transport during the September 2002 Antarctic Major Warming. Journal of the Atmospheric Sciences 62, 690-707. 
Manney, G., J. Sabutis, S. Pawson, M. Santee, B. Naujokat, R. Swinbank, M. Gelman, and W. Ebisuzaki (2003). Lower stratospheric temperature differences between meteorological analyses in two cold Arctic winters and their impact on polar processing studies. Journal of Geophysical Research 108(D5), 8328, doi:10.1029/2001JD001149.

Manney, G., R. Swinbank, S. Massie, M. Gelman, A. Miller, R. Nagatani, A. O'Neill, and R. Zurek (1996). Comparison of U.K. Meteorological Office and U.S. National Meteorological Center stratospheric analyses during northern and southern winter. JGR 101(D6), 10311-10334.

Manzini, E., B. Steil, C. Brühl, M. Giorgetta, and K. Krüger (2003). A new interactive chemistry-climate model: 2. Sensitivity of the middle atmosphere to ozone depletion and increase in greenhouse gases and implications for recent stratospheric cooling. Journal of Geophysical Research 108(D14), 4429, doi:10.1029/2002JD002977.

Marsh, D., J. Lamarque, A. Conley, and L. Polvani (2016). Stratospheric ozone chemistry feedbacks are not critical for the determination of climate sensitivity in CESM1(WACCM). Geophysical Research Letters 43, 39283934.

McIntyre, M. (1989). On the Antarctic Ozone Hole. Journal of Atmospheric and Terrestrial Physics 51(1), 29-43.

McIntyre, M. and T. Palmer (1983). Breaking planetary waves in the stratosphere. Nature 305, 593-600.

McIntyre, M. and T. Palmer (1984). The 'surf zone' in the stratosphere. Journal of Atmospheric and Terrestrial Physics 46(9), 825-849.

McLandress, C., T. Shepherd, S. Polavarapu, and S. Beagley (2012). Is Missing Orographic Gravity Wave Drag near 608S the Cause of the Stratospheric Zonal Wind Biases in Chemistry-Climate Models? Journal of the Atmospheric Sciences 69, 802-818.

McLandress, C., T. Shepherd, J. Scinocca, D. Plummer, M. Sigmond, A. Jonsson, and M. Reader (2011). Separating the Dynamical Effects of Climate Change and Ozone Depletion. Part II: Southern Hemisphere Troposphere. Journal of Climate 24, 1850-1868.

Mitchell, D. M., L. Montabone, S. Thomson, and P. Read (2015). Polar vortices on Earth and Mars: A comparative study of the climatology and 
variability from reanalyses. Quarterly Journal of the Royal Meteorological Society 141, 550-562.

Morgenstern, O., P. Braesicke, F. O’Connor, A. Bushell, C. Johnson, S. Osprey, and J. Pyle (2009). Evaluation of the new UKCA climate-composition model - Part 1: The stratosphere. Geoscientific Model Development 2(1).

Morgenstern, O., M. Hegglin, E. Rozanov, F. O'Connor, N. Abraham, H. Akiyoshi, A. Archibald, S. Bekki, N. Butchart, M. Chipperfield, M. Deushi, S. Dhomse, R. Garcia, S. Hardiman, L. Horowitz, P. Jöckel, B. Josse, D. Kinnison, M. Lin, E. Mancini, M. Manyin, M. Marchand, V. Marécal, M. Michou, L. Oman, G. Pitari, D. Plummer, L. Revell, D. Saint-martin, R. Schofield, A. Stenke, K. Stone, K. Sudo, T. Tanaka, S. Tilmes, Y. Yamashita, K. Yoshida, and G. Zeng (2017). Review of the global models used within phase 1 of the Chemistry-Climate Model Initiative (CCMI). Geoscientific Model Development 10, 639-671.

Nakamura, N. (1996). Two-dimensional mixing, edge formation, and permeability diagnosed in an area coordinate. Journal of the Atmospheric Sciences 53(11), 1524-1537.

Nakamura, N. (2008). Sensitivity of Global Mixing and Fluxes to Isolated Transport Barriers. Journal of the Atmospheric Sciences 65, 3800-3818.

Nakamura, N. and J. Ma (1997). Modified Lagrangian-mean diagnostics of the stratospheric polar vortices 2. Nitrous oxide and seasonal barrier migration in the cryogenic limb array etalon spectrometer and SKYHI general circulation model. Journal of Geophysical Research 102(D22), 2572125735 .

NASA (1976). U.S. Standard Atmosphere, 1976. Washington, D.C.: NASA.

Nash, E., P. Newman, J. Rosenfield, and M. Schoeberl (1996). An objective determination of the polar vortex using Ertel's potential vorticity. Journal of Geophysical Research 101(D5), 9471-9478.

Nathan, T. and E. Cordero (2007). An ozone-modified refractive index for vertically propagating planetary waves. JGR 112(D02105).

Neely, R., D. Marsh, K. Smith, S. Davis, and L. Polvani (2014). Biases in Southern Hemisphere climate trends induced by coarsely specifying the temporal resolution of stratospheric ozone. Geophysical Research Letters 41, 8602-8610. 
Newman, P. (2010). Chemistry and Dynamics of the Antarctic Ozone Hole. Washington, DC: American Geophysical Union.

Newman, P., L. Coy, S. Pawson, and L. Lait (2016). The anomalous change in the QBO in 2015-2016. Geophysical Research Letters.

Newman, P., J. Daniel, D. Waugh, and E. Nash (2007). A new formulation of equivalent effective stratospheric chlorine (EESC). Atmospheric Chemistry and Physics 7, 4537-4552.

Orr, A., T. Bracegirdle, J. Hosking, T. Jung, J. Haigh, and T. Phillips (2012). Possible dynamical mechanisms for southern hemisphere climate change due to the ozone hole. J. Atmos. Sci. 69, 2917-32.

Parrondo, M., M. Yela, M. Gil, P. von der Gathen, and H. Ochoa (2006). Mid-winter lower stratosphere temperatures in the Antarctic vortex: comparison between observations and ECMWF and NCEP operational models. Atmospheric Chemistry and Physics 7, 435-441.

Peacock, T. and G. Haller (2013). Lagrangian coherent structures: the hidden skeleton of fluid flows. Physics Today 66(2), 41-47.

Perlwitz, J. (2011). Tug of war on the jet stream. Nature Climate Change 1, 29-31.

Peters, G., R. Andrew, S. Solomon, and P. Friedlingstein (2015). Measuring a fair and ambitious climate agreement using cumulative emissions. Environmental Research Letters 10(105004).

Pfotzer, G. (1972). History of the Use of Balloons in Scientific Experiments. Space Science Reviews 13(2), 199-242.

Pierrehumbert, R. and H. Yang (1993). Global Chaotic Mixing on Isentropic Surfaces. Journal of the Atmospheric Sciences 50(15), 2462-2480.

Plumb, R. (1996). A "tropical pipe" model of stratospheric transport. Journal of Geophysical Research 101(D2), 3957-3972.

Plumb, R. (2010). Planetary Waves and the Extratropical Winter Stratosphere. Washington, DC: American Geophysical Union.

Plumb, R. and M. Ko (1992). Interrelationships between mixing ratios of long-lived stratospheric constituents. Journal of Geophysical Research 97(D9), 10145-10156. 
Plumb, R., D. Waugh, R. Atkinson, P. Newman, L. Lait, M. Schoeberl, E. Browell, A. Simmons, and M. Loewenstein (1994). Intrusions into the lower stratospheric Arctic vortex during the winter of 1991-1992. Journal of Geophysical Research 99(D1), 1089-1105.

Plumb, R., D. Waugh, and M. Chipperfield (2000). The effects of mixing on tracer relationships in the polar vortices. Journal of Geophysical Research 105(D8), 10047-10062.

Plumb, R. A. (2007). Tracer interrelationships in the stratosphere. Reviews of Geophysics 45(RG4005).

Podglajen, A., A. Hertzog, R. Plougonven, and B. Legras (2016). Lagrangian temperature and vertical velocity fluctuations due to gravity waves in the lower stratosphere. Geophysical Research Letters 43, 3543-3553.

Polvani, L. and R. A. Plumb (1992). Rossby Wave Breaking, Microbreaking, Filamentation, and Secondary Vortex Formation: The Dynamics of a Perturbed Vortex. Journal of the Atmospheric Sciences 49(6).

Polvani, L., M. Previdi, and C. Deser (2011). Large cancellation, due to ozone recovery, of future Southern Hemisphere atmospheric circulation trends. Geophysical Research Letters 38, L04707, doi:10.1029/2011GL046712.

Proffitt, M. H., K. K. Kelly, J. A. Powell, B. L. Gary, M. Loewenstein, J. R. Podolske, S. E. Strahan, and K. R. Chan (1989). Evidence for diabatic cooling and poleward transport within and around the 1987 Antarctic ozone hole. J. Geophys. Res. 16(813).

Rabier, F., A. Bouchard, E. Brun, A. Doerenbecher, S. Guedj, V. Guidard, F. Karbou, V. Peuch, L. El Amraoui, D. Puech, C. Genthon, G. Picard, M. Town, A. Hertzog, F. Vial, P. Cocquerez, S. Cohn, T. Hock, J. Fox, H. Cole, D. Parsons, J. Powers, K. Romberg, J. Vanandel, T. Deshler, J. Mercer, J. Haase, L. Avallone, L. Kalnajs, C. Mechoso, A. Tangborn, A. Pellegrini, Y. Frenot, J. Thépaut, A. Mcnally, G. Balsamo, and P. Steinle (2010). The Concordiasi project in Antarctica. Bulletin of the American Meteorological Society 91(1).

Randall, D., R. Wood, S. Bony, R. Colman, T. Fichefet, J. Fyfe, V. Kattsov, A. Pitman, J. Shukla, J. Srinivasan, R. Stouffer, A. Sumi, and K. Taylor (2007). Cilmate Models and Their Evaluation. Report, IPCC. 
Randel, W. and J. Cobb (1994). Coherent variations of monthly mean total ozone and lower stratospheric temperature. Journal of Geophysical Research 99(D3), 5433-5447.

Rayner, N., D. Parker, E. Horton, C. Folland, L. Alexander, and D. Rowell (2003). Global analyses of sea surface temperature, sea ice, and night marine air temperature since the late nineteenth century. JGR 108.

Revell, L., F. Tummon, A. Stenke, T. Sukhodolov, A. Coulon, E. Rozanov, H. Garny, V. Grewe, and T. Peter (2015). Drivers of the tropospheric ozone budget throughout the 21st century under the medium-high climate scenario RCP 6.0. Atmospheric Chemistry and Physics 15, 5887-5902.

Reynolds, R., T. Smith, C. Liu, D. Chelton, K. Casey, and M. Schlax (2007). Daily High-Resolution-Blended Analyses for Sea Surface Temperature. Journal of Climate 20, 5473-5496.

Riahi, K., S. Rao, V. Krey, C. Cho, V. Chirkov, G. Fischer, G. Kindermann, N. Nakicenovic, and P. Rafaj (2011). RCP 8.5-A scenario of comparatively high greenhouse gas emissions. Climatic Change 109, 33-57.

Ricaud, P., F. Lefèvre, G. Berthet, D. Murtagh, E. Llewellyn, G. Mégie, E. Kyrölä, G. Leppelmeier, H. Auvinen, C. Boonne, S. Brohede, D. Degenstein, J. de La Noë, E. Dupuy, L. El Amraoui, P. Eriksson, W. Evans, U. Frisk, R. Gattinger, F. Girod, C. Haley, S. Hassinen, A. Hauchecorne, C. Jimenez, E. Kyrö, N. Lautié, E. Le Flochmoën, N. Lloyd, J. McConnell, I. McDade, L. Nordh, M. Olberg, A. Pazmino, S. Petelina, A. Sandqvist, A. Seppälä, C. Sioris, B. Solheim, J. Stegman, K. Strong, P. Taalas, J. Urban, C. von Savigny, F. von Scheele, and G. Witt (2005). Polar vortex evolution during the 2002 Antarctic major warming as observed by the Odin satellite. Journal of Geophysical Research 110, D05302, doi:10.1029/2004JD005018.

Roeckner, E., G. Bäuml, L. Bonaventura, R. Brokopf, M. Esch, M. Giorgetta, S. Hagemann, I. Kirchner, L. Kornblueh, E. Manzini, A. Rhodin, U. Schlese, U. Schulzweida, and A. Tompkins (2003). The atmospheric general circulation model ECHAM 5. PART I: Model description. MaxPlanck-Institut für Meteorologie, Hamburg Report No. 349.

Roscoe, H., W. Feng, M. Chipperfield, M. Trainic, and E. Shuckburgh (2012). The existence of the edge region of the Antarctic stratospheric vortex. Journal of Geophysical Research 117(D04301), doi:10.1029/2011JD015940. 
Saha, S., S. Moorthi, H. Pan, X. Wu, J. Wang, S. Nadiga, P. Tripp, R. Kistler, J. En, D. Behringer, H. Liu, D. Stokes, R. Grumbine, G. Gayno, J. Wang, Y. Hou, H. Chuang, H. Juang, J. Sela, M. Iredell, R. Treadon, D. Kleist, P. T, D. Keyser, J. Derber, M. Ek, J. Meng, H. Wei, R. Yang, S. Lord, H. van den Dool, A. Kumar, W. Wang, C. Long, M. Iah, Y. Xue, B. Huang, J. Schemm, W. Ebisuzaki, R. Lin, P. Xie, M. Chen, S. Zhou, W. Higgins, C. Zou, Q. Liu, Y. Chen, Y. Han, L. Cucurull, R. Reynolds, G. Rutledge, and M. Goldberg (2010). The NCEP Climate Forecast System Reanalysis. Bulletin of the American Meteorological Society, 1015-1057.

Santer, B., T. Wigley, C. Mears, F. Wentz, S. Klein, D. Seidel, K. Taylor, P. Thorne, M. Wehner, P. Gleckler, J. Boyle, W. Collins, K. Dixon, C. Doutriaux, M. Free, Q. Fu, J. Hansen, G. Jones, R. Ruedy, T. Karl, J. Lanzante, G. Meehl, V. Ramaswamy, G. Russell, and G. Schmidt (2005). Amplification of Surface Temperature Trends and Variability in the Tropical Atmosphere. Science 309, 1551-1556.

Scaife, A., N. Butchart, C. Warner, and R. Swinbank (2002). Impact of a Spectral Gravity Wave Parameterization on the Stratosphere in the Met Office Unified Model. Journal of the Atmospheric Sciences 59, 1473-1489.

Schoeberl, M. and D. Hartmann (1991). The dynamics of the stratospheric polar vortex and its relation to springtime ozone depletions. Science 251, $46-52$.

Schoeberl, M., L. Lait, P. Newman, and J. Rosenfield (1992). The structure of the polar vortex. Journal of Geophysical Research 97(D8), 7859-1882.

Schoeberl, M. and P. Newman (1995). A muliple-level trajectory analysis of vortex filaments. Journal of Geophysical Research 100D12, 25801-25815.

Schoeberl, M. R., E. Jensen, A. Podglajen, L. Coy, C. Lodha, S. Candido, and R. Carver (2017). Gravity wave spectra in the lower stratosphere diagnosed from project loon balloon trajectories. JGR 122, 8517-8524.

Screen, J. and I. Simmonds (2011). Erroneous Arctic Temperature Trends in the ERA-40 Reanalysis: A Closer Look. Journal of Climate 24, 26202627.

Serra, M., P. Sathe, F. Beron-Vera, and G. Haller (2017). Uncovering the Edge of the Polar Vortex. Journal of the Atmospheric Sciences 74, 3871-3885. 
Shepherd, T. (2003). Large-Scale Atmospheric Dynamics for Atmospheric Chemists. Chem. Rev. 103, 4509-4531.

Shuckburgh, E., F. D'Ovidio, and B. Legras (2009). Local Mixing Events in the Upper Troposphere and Lower Stratosphere. Part II: Seasonal and Interannual Variability. Journal of the Atmospheric Sciences 66, 36953706.

Smith, M. and A. McDonald (2014). A quantitative measure of polar vortex strength using the function M. Journal of Geophysical Research 119, $5966-5985$.

Sobel, A. H., R. A. Plumb, and D. W. Waugh (1997). Methods of Calculating Transport across the Polar Vortex Edge. Journal of the Atmospheric Sciences 54(18), 2241-2260.

Solomon, S., R. Garcia, F. Rowland, and D. Wuebbles (1986). On the depletion of Antarctic ozone. Nature 321, 755-758.

Solomon, S., R. Garcia, and F. Stordal (1985). Transport Processes and Ozone Perturbations. Journal of Geophysical Research 90(D7), 1298112989 .

Solomon, S., D. Ivy, D. Kinnison, M. Mills, R. Neely, and A. Schmidt (2016). Emergence of healing in the Antarctic ozone layer. Science.

SPARC (2010). SPARC CCMVal Report on the Evaluation of ChemistryClimate Models. Report, World Climate Research Programme.

Stenke, A., M. Schraner, E. Rozanov, T. Egorova, B. Luo, and T. Peter (2013). SOCOLv3 version 3.0 chemistry-climate model: description, evaluation, and implications from an advanced transport algorithm. Geoscientific Model Development 6, 1407-1427.

Stohl, A., O. Cooper, and P. James (2004). A Cautionary Note on the Use of Meteorological Analysis Fields for Quantifying Atmospheric Mixing. Journal of the Atmospheric Sciences 61, 1446-1453.

Strahan, S. and B. Polansky (2006). Meteorological implementation issues in chemistry and transport models. Atmospheric Chemistry and Physics 6, 2895-2910.

Struthers, H., G. Bodeker, J. Austin, S. Bekki, I. Cionni, M. Dameris, M. Giorgetta, V. Grewe, F. Lefèvre, F. Lott, E. Manzini, T. Peter, 
E. Rozanov, and M. Schraner (2009). The simulation of the Antarctic ozone hole by chemistry-climate models. Atmospheric Chemistry and Physics 9, 6363-6376.

Sutton, R., H. Maclean, R. Swinbank, A. O’Neill, and F. Taylor (1994). Highresolution stratospheric tracer fields estimated from satellite observations using lagrangian trajectory calculations. Journal of the Atmospheric Sciences 51(20), 2995-3005.

Thompson, D., M. Baldwin, and S. Solomon (2005). Stratosphere-Troposphere coupling in the Southern Hemisphere. Journal of the Atmospheric Sciences 62, 708-715.

Thompson, D. and S. Solomon (2002). Interpretation of recent southern hemisphere climate change. Science 296, 895-899.

Thompson, D. and S. Solomon (2009). Understanding Recent Stratospheric Climate Change. Journal of Climate 22, 1934-1943.

Thompson, D., S. Solomon, P. Kushner, M. England, K. Grise, and D. Karoly (2011). Signatures of the Antarctic ozone hole in Southern Hemisphere surface climate change. Nature Geoscience, doi: 10.1038/NGEO1296.

Thompson, D. and J. Wallace (2000). Annular modes in the extratropical circulation. Part I: month-to-month variability. Journal of Climate 13, $1000-1016$.

Tuck, A. F. (1989). Synoptic and chemical evolution of the Antarctic vortex in late winter and early spring, 1987. J. Geophys. Res. 16(737).

Vosper, S. (2015). Mountain waves and wakes generated by South Georgia: implications for drag parametrization. Quarterly Journal of the Royal Meteorological Society 141(692), 2813-2827.

Walters, D., A. Baran, I. Boutle, M. Brooks, P. Earnshaw, J. Edwards, K. Furtado, P. Hill, A. Lock, J. Manners, C. Morcrette, J. Mulcahy, C. Sanchez, C. Smith, R. Stratton, W. Tennant, L. Tomassini, K. Van Weverberg, S. Vosper, M. Willett, J. Browse, A. Bushell, M. Dalvi, R. Essery, N. Gedney, S. Hardiman, B. Johnson, C. Johnson, A. Jones, G. Mann, S. Milton, H. Rumbold, A. Sellar, M. Ujiie, M. Whitall, K. Williams, and M. Zerroukat (2018). The Met Office Unified Model Global Atmosphere 7.0/7.1 and JULES Global Land 7.0 configurations. Geoscientific Model Development In review. 
Walters, D., M. Best, A. Bushell, D. Copsey, J. Edwards, P. Falloon, C. Harris, A. Lock, J. Manners, C. Morcrette, M. Roberts, R. Stratton, S. Webster, J. Wilkinson, M. Willett, I. Boutle, P. Earnshaw, P. Hill, C. Maclachlan, G. Martin, W. Moufouma-Okia, M. Palmer, J. Petch, G. Rooney, A. Scaife, and K. Williams (2011). The Met Office Unified Model Global Atmosphere 3.0/3.1 and JULES Global Land 3.0/3.1 configurations. Geoscientific Model Development 4(4), 919-941.

Walters, D., I. Boutle, M. Brooks, T. Melvin, R. Stratton, S. Vosper, H. Wells, K. Williams, N. Wood, T. Allen, A. Bushell, D. Copsey, P. Earnshaw, J. Edwards, M. Gross, S. Hardiman, C. Harris, J. Heming, N. Klingaman, R. Levine, J. Manners, G. Martin, S. Milton, M. Mittermaier, C. Morcrette, T. Riddick, M. Roberts, C. Sanchez, P. Selwood, A. Stirling, C. Smith, D. Suri, W. Tennant, P. Vidale, J. Wilkinson, M. Willett, S. Woolnough, and P. Xavier (2017). The Met Office Unified Model Global Atmosphere 6.0/6.1 and JULES Global Land 6.0/6.1 configurations. Geoscientific Model Development 10(4).

Waugh, D. (1997). Elliptical diagnostics of stratospheric polar vortices. Quarterly Journal of the Royal Meteorological Society 123, 1725-1748.

Waugh, D., L. Oman, P. Newman, R. Stolarski, S. Pawson, J. Nielsen, and J. Perlwitz (2009). Effect of zonal asymmetries in stratospheric ozone on simulated Southern Hemisphere climate trends. Geophysical Research Letters 36, L18701, doi:10.1029/2009GL040419.

Waugh, D. and R. Plumb (1994). Contour advection with surgery: A technique for investigating finescale structure in tracer transport. Journal of the Atmospheric Sciences 51(4), 530-540.

Waugh, D. and L. Polvani (2010). Stratospheric Polar Vortices.

Waugh, D. and W. Randel (1999). Climatology of Arctic and Antarctic polar vortices using elliptical diagnostics. Journal of the Atmospheric Sciences 56, 1594-1613.

Webb, M., F. Lambert, and J. Gregory (2012). Origins of differences in climate sensitivity, forcing and feedback in climate models. Climate Dynamics 40(3-4), 677-707.

Webster, S., A. Brown, D. Cameron, and C. Jones (2003). Improvements to the representation of orography in the Met Office Unified Model. Quarterly Journal of the Royal Meteorological Society 129(591), 1989-2010. 
Whelan, E., E. Gleeson, and J. Hanley (2018). An Evaluation of MÉRA, a High-Resolution Mesoscale Regional Reanalysis. Journal of Applied Meteorology and Climatology Vol.57(9), 2179-2196.

WMO (2010). Scientific Assessment of Ozone Depletion: 2010.

WMO (2015). Antarctic Ozone Bulletin.

Wood, N., A. Staniforth, A. White, T. Allen, M. Diamantakis, M. Gross, T. Melvin, C. Smith, S. Vosper, M. Zerroukat, and J. Thuburn (2014). An inherently mass-conserving semi-implicit semi-Lagrangian discretization of the deep-atmosphere global non-hydrostatic equations. Quarterly Journal of the Royal Meteorological Society 140(682), 1505-1520.

Xu, J., H.-L. Liu, W. Yuan, A. Smith, R. G. Roble, C. Mertens, J. Russell, and M. Mlynczak (2007). Mesopause structure from Thermosphere, Ionosphere, Mesosphere, Energetics, and Dynamics (TIMED)/Sounding of the Atmosphere Using Broadband Emission Radiometry (SABER). Journal of Geophysical Research 112(D9). 